\title{
WestVirginiaUniversity
}

THE RESEARCH REPOSITORY @ WVU

Graduate Theses, Dissertations, and Problem Reports

2013

\section{Analysis of GPS Abnormal Conditions within Fault Tolerant Control Laws}

Gahssan Al-Sinbol

West Virginia University

Follow this and additional works at: https://researchrepository.wvu.edu/etd

\section{Recommended Citation}

Al-Sinbol, Gahssan, "Analysis of GPS Abnormal Conditions within Fault Tolerant Control Laws" (2013). Graduate Theses, Dissertations, and Problem Reports. 4948.

https://researchrepository.wvu.edu/etd/4948

This Thesis is protected by copyright and/or related rights. It has been brought to you by the The Research Repository @ WVU with permission from the rights-holder(s). You are free to use this Thesis in any way that is permitted by the copyright and related rights legislation that applies to your use. For other uses you must obtain permission from the rights-holder(s) directly, unless additional rights are indicated by a Creative Commons license in the record and/ or on the work itself. This Thesis has been accepted for inclusion in WVU Graduate Theses, Dissertations, and Problem Reports collection by an authorized administrator of The Research Repository @ WVU. For more information, please contact researchrepository@mail.wvu.edu. 
Analysis of GPS Abnormal Conditions within Fault Tolerant Control Laws

$$
\text { Ghassan Al-Sinbol }
$$

Thesis submitted to the Benjamin M. Statler College of Engineering \& Mineral Resources

\author{
at West Virginia University \\ in partial fulfillment of the requirements \\ for the degree of \\ Master of Science \\ in \\ Mechanical Engineering
}

\author{
Mario G. Perhinschi, Ph.D., Chair \\ Larry E. Banta, Ph.D. \\ Srikanth Gururajan, Ph.D.
}

Department of Mechanical and Aerospace Engineering

Morgantown, West Virginia

2013

Keywords: GPS; GPS failures; GPS error model; fault tolerant control laws; flight simulation 


\section{ABSTRACT \\ Analysis of GPS Abnormal Conditions within Fault Tolerant Control Laws}

\section{Ghassan Al-Sinbol}

The Global Position System (GPS) is a critical element for the functionality of autonomous flying vehicles. The GPS operation at normal and abnormal conditions directly impacts the trajectory tracking performance of the autonomous Unmanned Aerial Vehicles (UAVs) controllers. The effects of GPS parameter variation must be well understood and user-friendly computational tools must be developed to facilitate the design and evaluation of fault tolerant control laws.

This thesis presents the development of a simplified GPS error model in Matlab/Simulink and its use performing a sensitivity analysis of GPS parameters effect under system normal and abnormal operation on different UAV trajectory tracking controllers. The model statistically generates position and velocity errors, simulates the effect of GPS satellite configuration on the position and velocity measurement accuracy, and implements a set of failures to the GPS readings. The model and its graphical user interface was integrated within the WVU UAV simulation environment as a masked Simulink block. The effects on the controllers' trajectory tracking performance of the following GPS parameters were investigated within normal operation ranges and outside: time delay, update rate, error standard deviation, bias, and major position and velocity failures. Several sets of control laws with fixed and adaptive parameters and of different levels of complexity have been used in this investigation. A complex performance index formulated in terms of tracking errors and control activity was used for control laws performance evaluation. The composition of various metrics within the performance index was performed using fixed and variable weights depending on the local characteristics of the commanded trajectory.

This study has revealed that GPS error parameters have a significant impact on control laws performance. The proposed GPS model has proved to be a valuable, flexible tool for testing and evaluation of the fault tolerant capabilities of autonomous flight control laws. 


\section{ACKNOWLEDGEMENTS}

I would like to thank Dr. Mario Perhinschi, my research advisor, for his support, guidance, patience, and teachings throughout my education. I greatly appreciate all that he has done for me and my education.

I also would like to thank Dr. Srikanth Gururajan, for providing me with helpful feedback throughout our discussions about this graduate project. I greatly appreciate his help that significantly improved my work. I would like to also thank my other committee member, Dr. Larry Banta for his great help and support.

I also would like to thank Mr. Ahmed Khalil who had a hard time correcting and revising my thesis. I also want to thank Brenton Wilburn. Brent, I really appreciate your help and your priceless time you spend helping me out.

My special thanks go to the Higher Committee for Education Development in Iraq (HCED) general director and staff for making this possible through providing me with the scholarship and all support I needed through my study.

To my mother, you are the greatest woman in my life. To my wife, Haneen Kareem, your support your love will never be forgotten. To my family, I could not make it without your support. To Dr. Khalil, Dr. Alshallah Raja, and Susan, you all truly became my second family, thank you for everything. To my friends, thank you all for your great support. 


\section{TABLE OF CONTENTS}

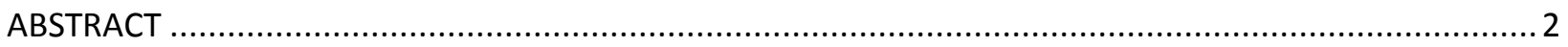

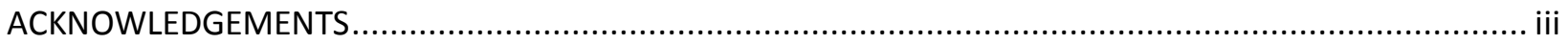

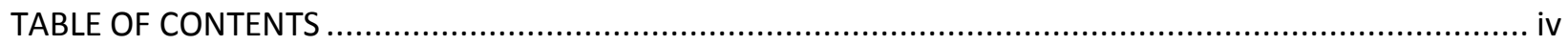

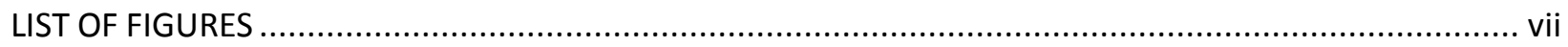

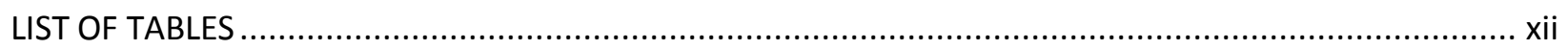

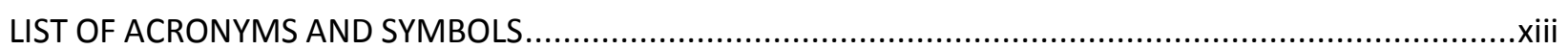

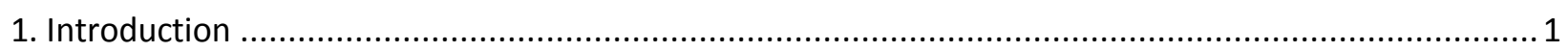

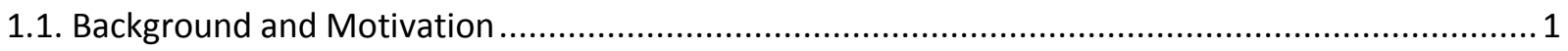

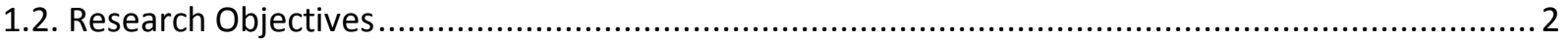

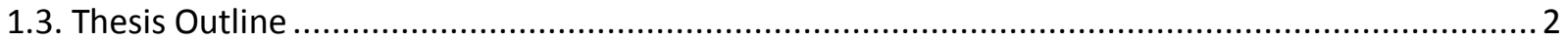

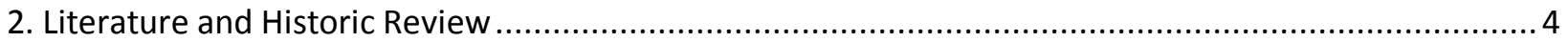

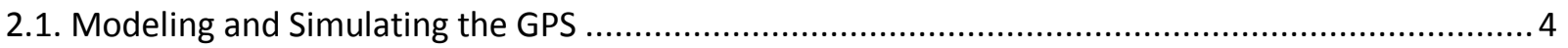

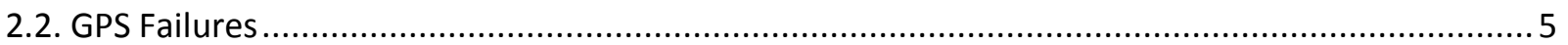

2.3. The Use of International GNSS Service's (IGS) Data .......................................................... 5

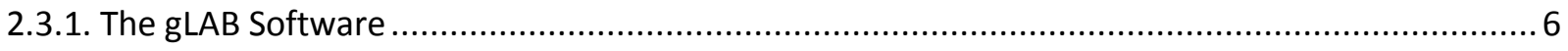

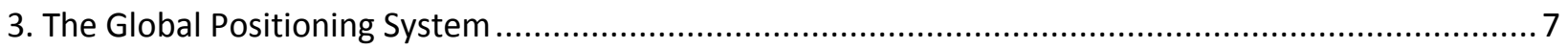

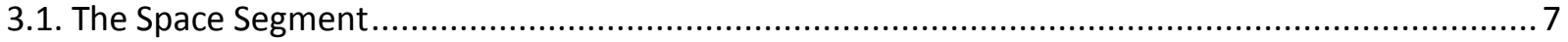

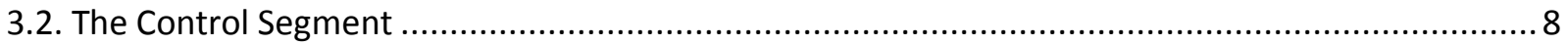

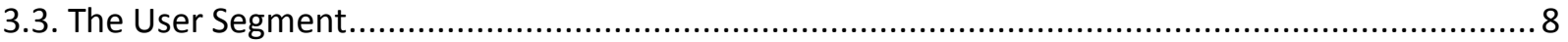

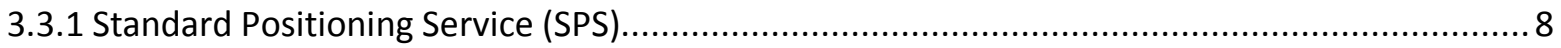

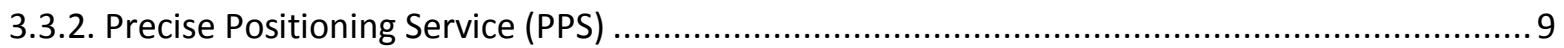

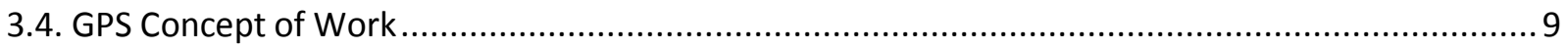

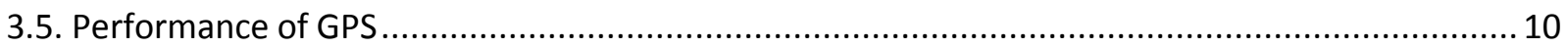

3.5.1. Satellite Clock and Ephemeris Errors …...................................................................... 10

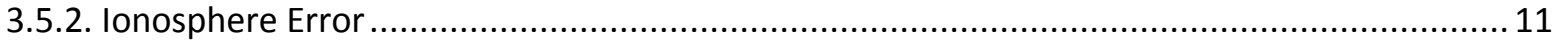

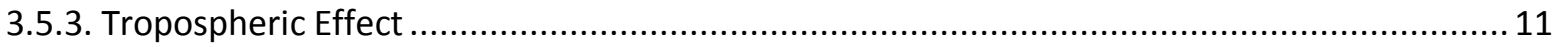

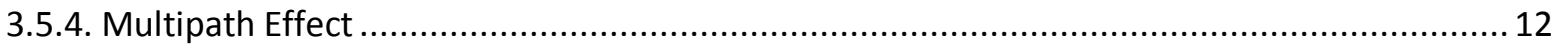

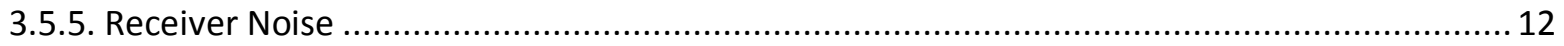

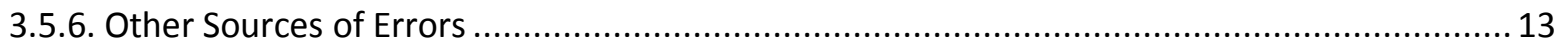

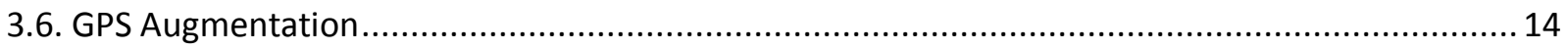

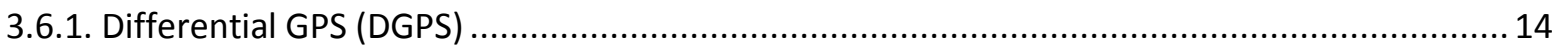

3.7. GPS Position and Velocity at Normal and Abnormal Conditions............................................. 16 


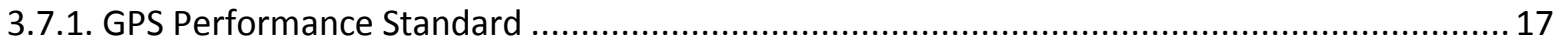

3.7.2. Position, Velocity, and Time (PVT) Accuracy Estimation ................................................. 20

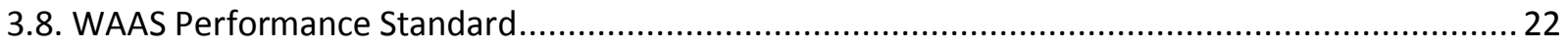

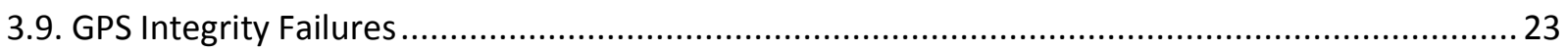

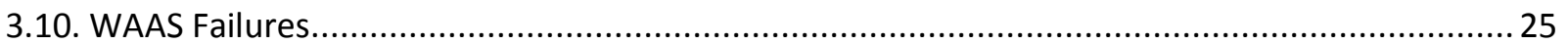

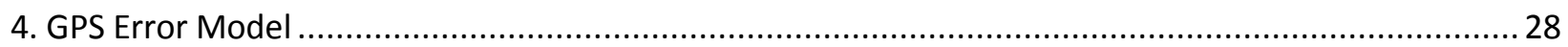

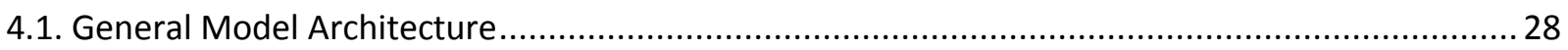

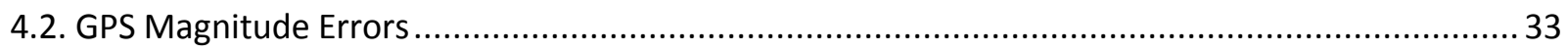

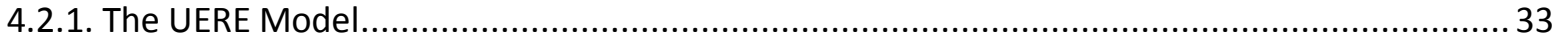

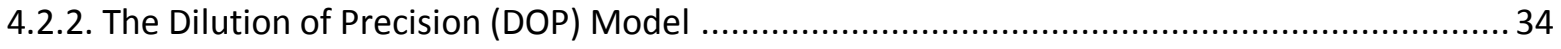

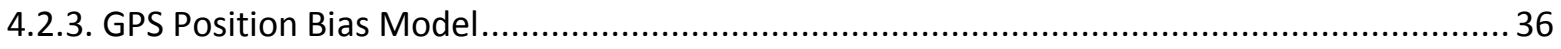

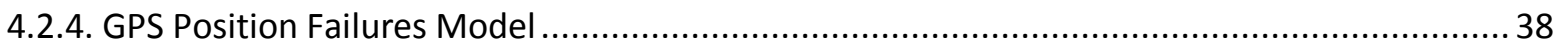

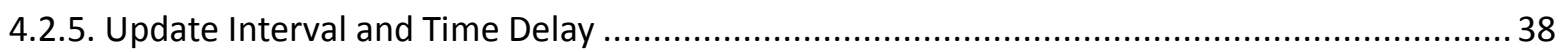

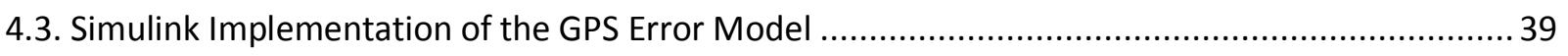

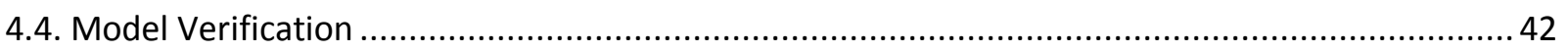

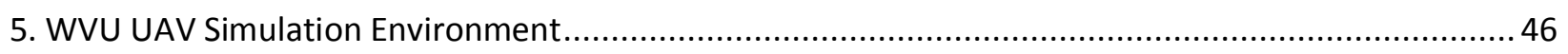

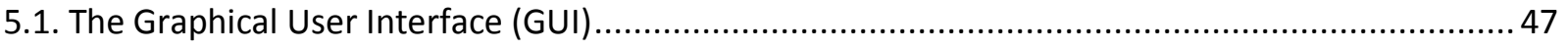

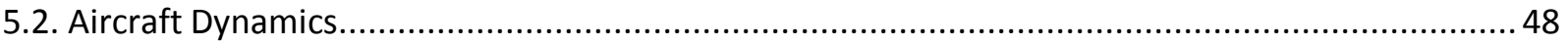

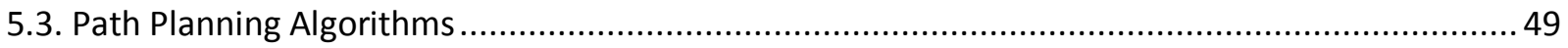

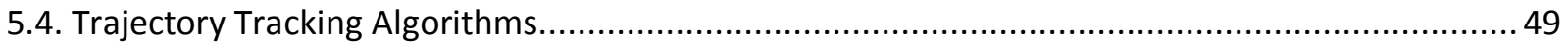

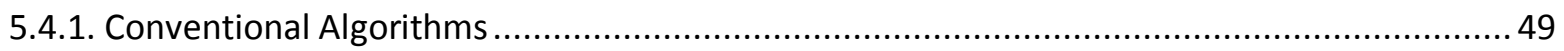

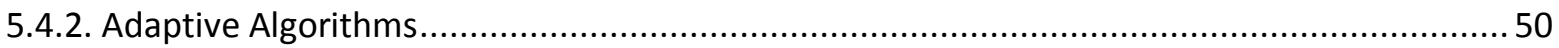

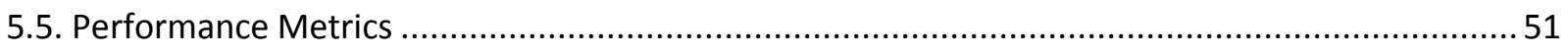

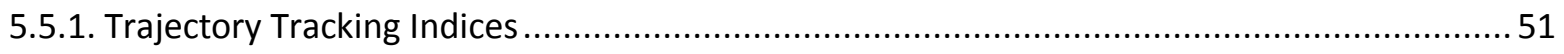

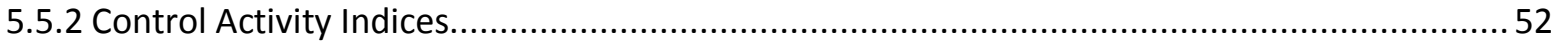

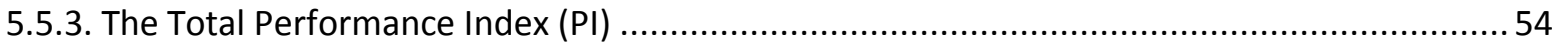

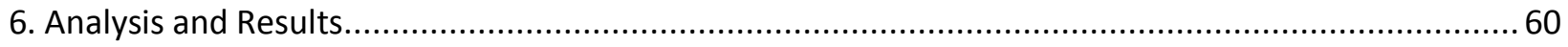

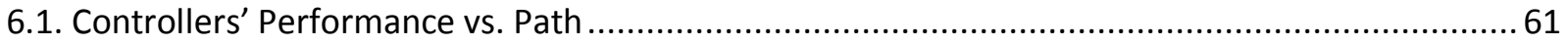

6.2. Variable Weight vs. Fixed Weight Performance Algorithm..................................................... 65

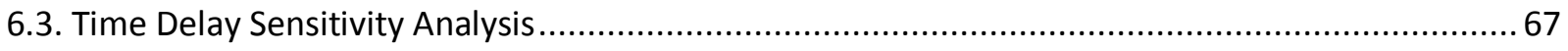

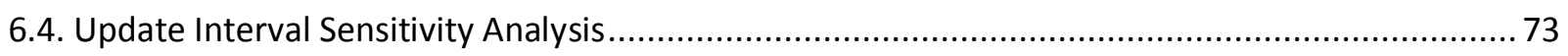

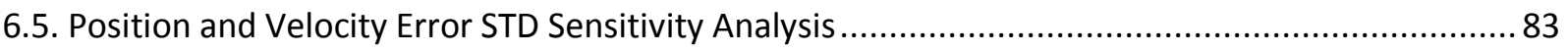

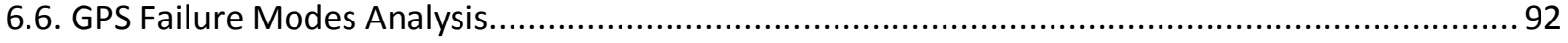


6.6.1. Noise Sensitivity Analysis.

6.6.2. Step Sensitivity Analysis

6.6.3. Ramp Sensitivity Analysis .... 100

6.6.4. Sinusoidal Sensitivity Analysis 104

6.7. Analysis Summary...... 108

7. Conclusions and Future Work . 109

8. Bibliography ..... 111 


\section{LIST OF FIGURES}

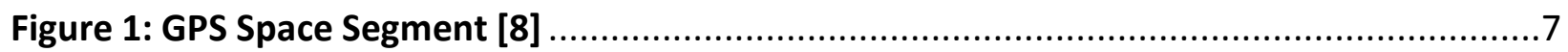

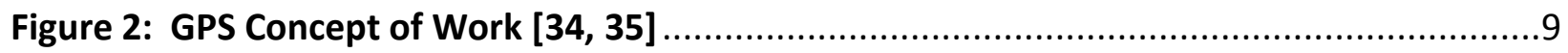

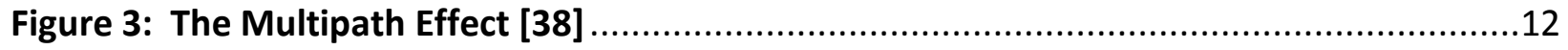

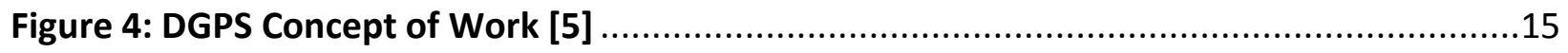

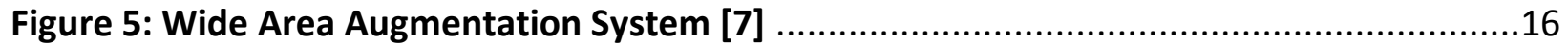

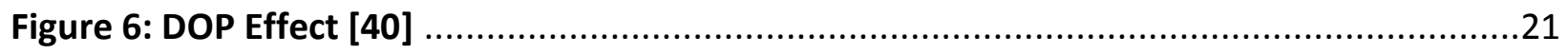

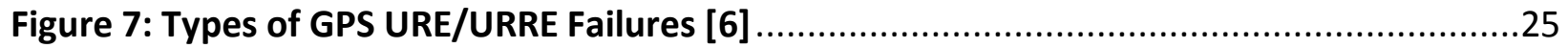

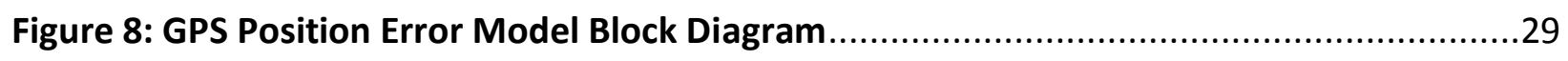

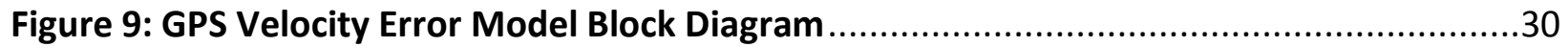

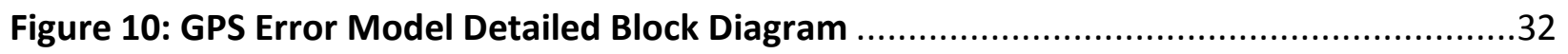

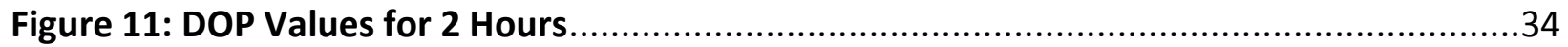

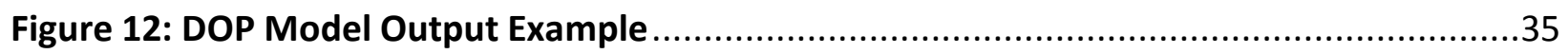

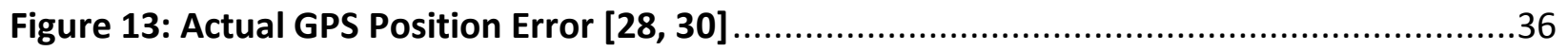

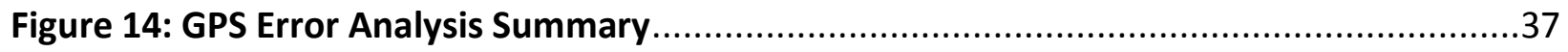

Figure 15: GPS Error Model Simulink Implementation .....................................................

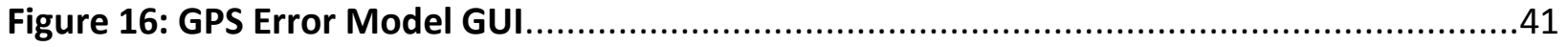

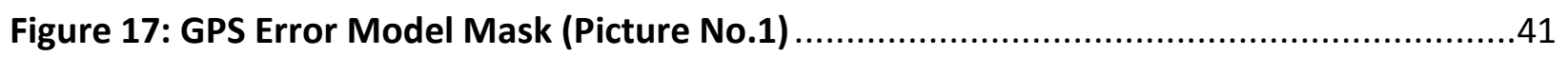

Figure 18: GPS Error Model Mask (Picture No.2) .............................................................42

Figure 19: GPS Error Model Position Error Example .....................................................4

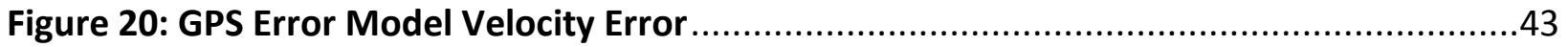

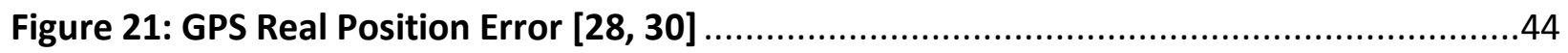

Figure 22: GPS Error Model Generated Position Error.......................................................44

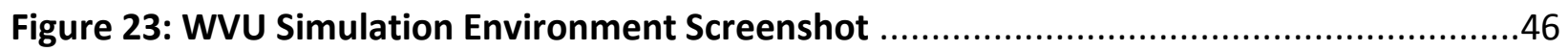

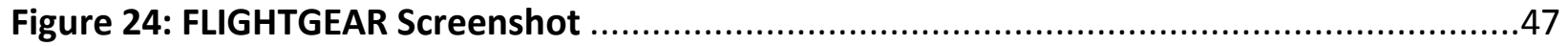

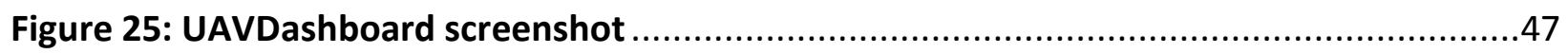

Figure 26: WVU UAV Simulation Environment GUI ..........................................................48

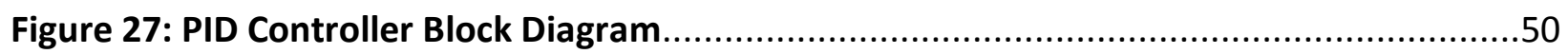


Figure 28: Actual and Commanded Trajectory for a Controller with "Good" Performance Index .56

Figure 29: Controller Surface Deflection for a Controller with “Good” Performance Index .....56 Figure 30: Actual and Commanded Trajectory for a Controller with “Adequate" Performance Index. .57

Figure 31: Controller Surface Deflection for a Controller with “Adequate" Performance Index .57

Figure 32: Actual and Commanded Trajectory for a Controller with "Poor" Performance Index .58

Figure 33: Controller Surface Deflection for a Controller with “Poor" Performance Index ......58

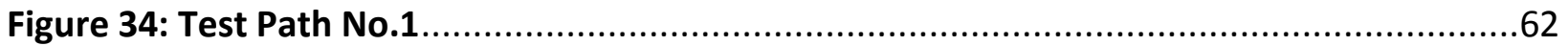

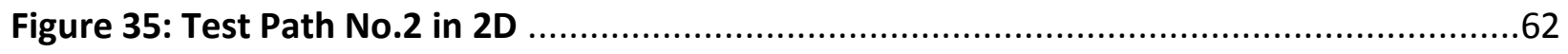

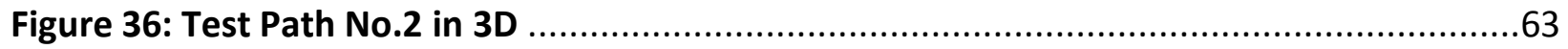

Figure 37: Controllers' PI vs. Time Delay (Path No.1 Analysis) ......................................63

Figure 38: Controllers' PI vs. Time Delay (Path No.2 Analysis) ....................................64

Figure 39: Controllers' PI vs. Update Interval (Path No.1 Analysis) .................................64

Figure 40: Controllers' PI vs. Update Interval Analysis (Path No.2 Analysis) ........................65

Figure 41: Controllers' PI vs. Position Noise Error STD (Using Variable Weight Algorithm) ......66

Figure 42: Controllers' PI vs. Position Noise Error STD (Using Fixed Weight Algorithm) ..........66

Figure 43: Controllers' TTI vs. Position Noise Error STD .................................................67

Figure 44: Controllers' CAI vs. Position Noise Error STD ................................................67

Figure 45: Controllers Performance Index vs. Time Delay ...........................................68

Figure 46: Controllers' TTI Index vs. Time Delay .........................................................70

Figure 47: Controllers' CAI Index vs. Time Delay .....................................................71

Figure 48: Commanded and Actual Trajectory for Position PID at $0.54 \mathrm{sec}$ Time Delay ..........72

Figure 49: Commanded and Actual Trajectory for Adaptive Outer at 0.54 sec Time Delay ......72 Figure 50: Controller Surfaces Deflections for Position PID at $0.54 \mathrm{sec}$ Time Delay ...............73

Figure 51: Controller Surfaces Deflections for Adaptive Outer at 0.54 sec Time Delay ...........73

Figure 52: Controllers Performance Index vs. Position and Velocity Update Interval .............74 
Figure 53: Controllers' TTI Index vs. Position and Velocity Update Interval ........................75

Figure 54: Controllers' CAI Index vs. Position and Velocity Update Interval .........................76

Figure 55: Commanded and Actual Trajectories for Position PID at $0.44 \mathrm{sec}$ Position and

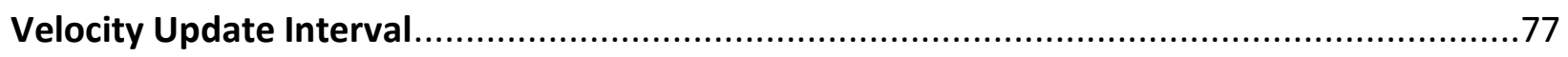

Figure 56: Commanded and Actual Trajectories for NLDI extended at $0.44 \mathrm{sec}$ Position and

Velocity Update Interval.

Figure 57: Controller Surfaces Deflections Position PID at 0.44 sec Position and Velocity

Update Interval .78

Figure 58: Controller Surfaces Deflections NLDI Extended at $0.44 \mathrm{sec}$ Position and Velocity

Update Interval 78

Figure 59: Controllers' Performance Index vs. Velocity Update Interval .............................79

Figure 60: Controllers' TTI Index vs. Velocity Update Interval .......................................80

Figure 61: Controllers' CAI Index vs. Velocity Update Interval ....................................80

Figure 62: Commanded and Actual Trajectories for Adaptive Position PID at 0.98 sec Velocity

Update Interval

Figure 63: Commanded and Actual Trajectories for NLDI Extended at $0.98 \mathrm{sec}$ Velocity Update Interval. .81

Figure 64: Controller Surface Deflection for Adaptive Position PID at $0.98 \mathrm{sec}$ Velocity Update Interval . .82

Figure 65: Controller Surface Deflection for NLDI Extended at $0.98 \mathrm{sec}$ Velocity Update Interval .82

Figure 66: Controllers' Performance Index vs. Position Error STD ....................................83

Figure 67: Controllers' TTI Index vs. Position Error STD ..................................................84

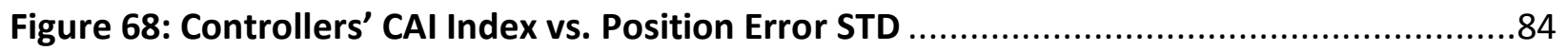

Figure 69: Controllers' Performance Index vs. Velocity Error STD ..................................86

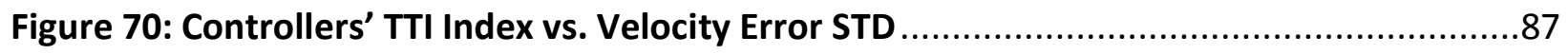

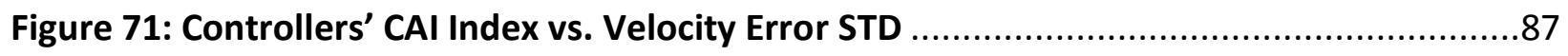

Figure 72: Controllers' Performance Index vs. Position and Velocity Error STD (Case 1) ..........88

Figure 73: Controllers' Performance Index vs. Position and Velocity Error STD (Case 2) ..........89 
Figure 74: Controllers' Performance Index vs. Position and Velocity Error STD (Case 3) ..... .89

Figure 75: Commanded and Actual Trajectories for Adaptive Position PID at 2 (m) STD Position Noise, and $0.6(\mathrm{~m} / \mathrm{s})$ STD Velocity Noise .90

Figure 76: Commanded and Actual Trajectories for NLDI Extended at 2 (m) STD Position Noise, and $0.6(\mathrm{~m} / \mathrm{s})$ STD Velocity Noise 91

Figure 77: Controller Surface Deflection for Adaptive Position PID at 2 (m) STD Position Noise, and $0.6(\mathrm{~m} / \mathrm{s})$ STD Velocity Noise .91

Figure 78: Controller Surface Deflection for NLDI Extended at 2 (m) STD Position Noise, and $0.6(\mathrm{~m} / \mathrm{s})$ STD Velocity Noise .92

Figure 79: Controllers' Performance Index vs. Measurement Noise for $12 \mathrm{sec} . . . \ldots \ldots \ldots \ldots \ldots . . . . . .93$

Figure 80: Controllers' Performance Index vs. Measurement Noise for 25 sec ...................93

Figure 81: Controllers' Performance Index vs. Measurement Noise for $\mathbf{5 0 ~ s e c ~ . . . . . . . . . . . . . . . . . . . 9 4 ~}$

Figure 82: Controllers' Performance Index vs. Measurement Noise for $75 \mathrm{sec} . . . \ldots \ldots \ldots \ldots \ldots . . . . . .94$

Figure 83: Controllers' Performance Index vs. Measurement Bias for $12 \mathrm{sec} . \ldots \ldots \ldots \ldots \ldots \ldots \ldots . . . . . . . . . .66$

Figure 84: Controllers' TTI vs. Measurement Bias for 12 sec .....................................96

Figure 85: Controllers' CAI vs. Measurement Bias for 12 sec....................................97

Figure 86: Controllers' Performance Index vs. Measurement Bias for $\mathbf{2 5}$ sec.....................98

Figure 87: Controllers' Performance Index vs. Measurement Bias for $\mathbf{5 0}$ sec......................98

Figure 88: Controllers' Performance Index vs. Measurement Bias for 75 sec.....................99

Figure 89: Controllers' Performance Index vs. Ramp Measurement Error for $12 \mathrm{sec} . . . \ldots \ldots . . . .100$

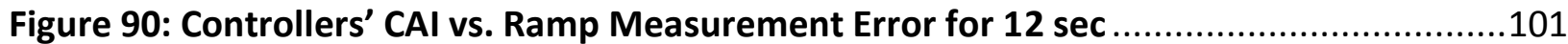

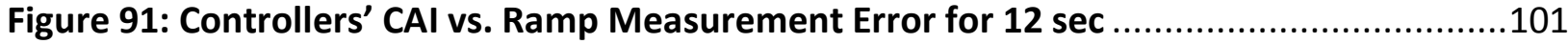

Figure 92: Controllers' Performance Index vs. Ramp Measurement Error for 25 sec ...........102

Figure 93: Controllers' Performance Index vs. Ramp Measurement Error for $50 \mathrm{sec} . . . \ldots \ldots \ldots . . .102$

Figure 94: Controllers' Performance Index vs. Ramp Measurement Error for 75 sec ...........103

Figure 95: Controllers Performance Index vs. Sinusoidal Measurement Error for $12 \mathrm{sec} . . . . . .104$

Figure 96: Controllers TTI vs. Sinusoidal Measurement Error for $12 \mathrm{sec}$...........................105

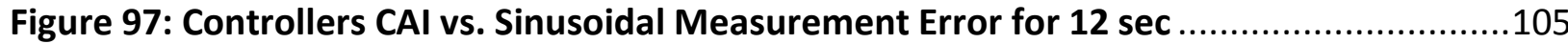

Figure 98: Controllers TTI vs. Sinusoidal Measurement Error for 25 sec ..........................106 
Figure 99: Controllers TTI vs. Sinusoidal Measurement Error for $50 \mathrm{sec}$ 106

Figure 100: Controllers TTI vs. Sinusoidal Measurement Error for $75 \mathrm{sec}$. 


\section{LIST OF TABLES}

Table 1: Error Sources Contribution to GPS UERE Budget[5] ..........................................13

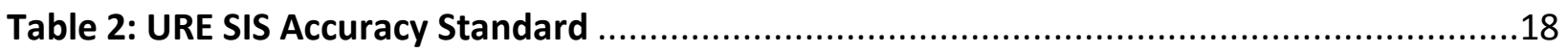

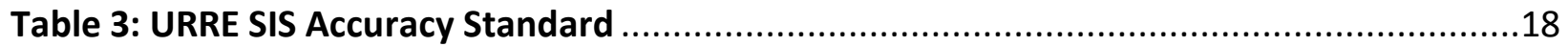

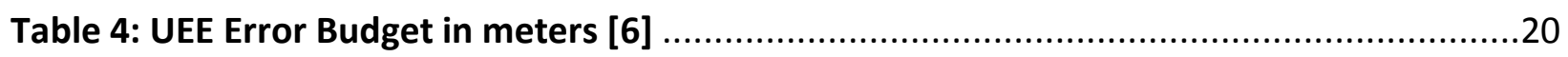

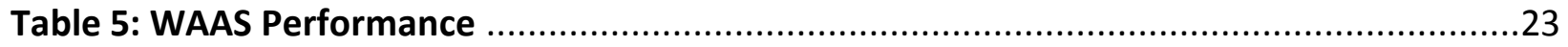

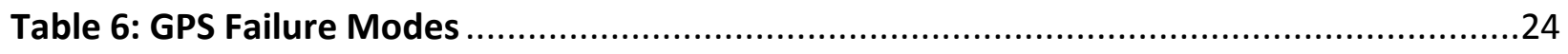

Table 7: WAAS Navigation Performance Requirements ............................................27

Table 8: Statistical Comparison between Actual and Regenerated GPS Position Error ...........45

Table 9: GPS Error Model Reference Parameters ........................................................60

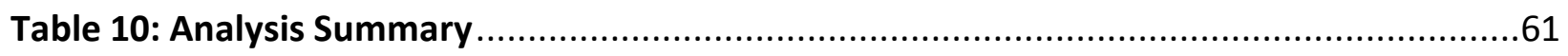

Table 11: Best and Worst Controllers in the Presence of Time Delays ...............................68

Table 12: Percentage Decrease in PI Due to Time Delay..................................................69

Table 13: Best and Worst Controllers for Different Update Interval Values..........................74

Table 14: Update Interval and Time Delay Results Comparison .....................................75

Table 15: Position and Velocity Errors STD Analysis Summery .....................................83

Table 16: Step Sensitivity Analysis Summary .............................................................99

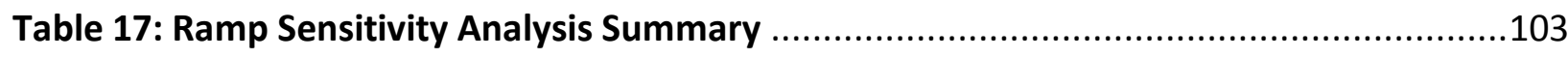

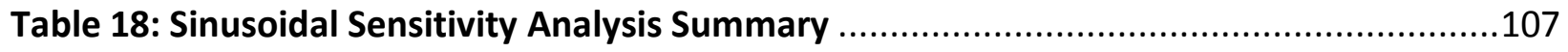

Table 19: Ranges for Good, Adequate, and Poor Performances ......................................108 


\section{LIST OF ACRONYMS AND SYMBOLS}

\begin{tabular}{|c|c|}
\hline AOD & Age Of Data \\
\hline $\mathrm{C} / \mathrm{A}$ & Coarse Acquisition \\
\hline CAI & Controller Activity Index \\
\hline DOP & Dilution Of Precision \\
\hline FAA & Federal Aviation Administration \\
\hline GNSS & Global Navigation Satellite System \\
\hline GPS & Global Positioning System \\
\hline GUI & Graphical User Interface \\
\hline HDOP & Horizontal Dilution Of Precision \\
\hline IGS & International GNSS Service \\
\hline PI & Performance Index \\
\hline PPS & Precise Positioning Service \\
\hline PVT & Position, Velocity, and Time \\
\hline SBAS & Satellite Based Augmentation System \\
\hline SIS & Signal In Space \\
\hline SPS & Standard Positioning Service \\
\hline STD & Standard Deviation \\
\hline SV & Single Vehicle \\
\hline TOA & Time of Arrival \\
\hline TTA & Time To Alert \\
\hline TTA & Time To Alert \\
\hline TTI & Trajectory Tracking Index \\
\hline UAVs & Unmanned Arial Vehicles \\
\hline UERE & User Equivalent Range Error \\
\hline UERE & User Equivalent Range Error \\
\hline
\end{tabular}




$\begin{array}{ll}\text { UERRE } & \text { User Equivalent Range Rate Error } \\ \text { URA } & \text { User Range Accuracy } \\ \text { URE } & \text { User Range Error } \\ \text { UTC } & \text { Universal Coordinated Time } \\ \text { VDOP } & \text { Vertical Dilution Of Precision } \\ \text { WAAS } & \text { Wide Area Augmentation System } \\ \text { WMS } & \text { WAAS Master Station } \\ \text { WRS } & \text { WAAS Reference Station } \\ \text { WVU } & \text { West Virginia University }\end{array}$




\section{Introduction}

\subsection{Background and Motivation}

The use of the Unmanned Arial Vehicles (UAVs) is becoming increasingly popular in military and civil applications. This popularity comes from the UAV ability to fly autonomously and perform a wide range of repetitive, long, tedious tasks with minimum human operator interaction [1]. The UAVs utilize different control algorithms for the purpose of autonomous flight such as trajectory tracking algorithms, or flight path stabilization algorithms [2].

The trajectory tracking algorithms are expected to fly an aircraft from one point to another on a pre-defined path, as well as preserve the aircraft safety in abnormal flight conditions caused by sensor failures, mechanical failures, or equipment deterioration. The trajectory tracking algorithms utilize a set of sensors to determine the next control action needed to preserve the UAV safety on the specified path. Most of the UAVs depend on the Global Positioning System (GPS) [3] or the similar Global Navigation Satellite System (GNSS) [4] to precisely determine their position and velocity at each point on the trajectory.

The GPS is a satellite based navigation system designed to provide precise position, velocity, and time (PVT) information to civil and military users all over the world [5]. The system is capable of providing up to centimeter-level accuracies under nominal conditions. Unfortunately, the system is susceptible to different source of errors such as atmosphere effect, signal attenuation, and multipath signals that degrade the system range accuracy up to 7.8 meters at a $95 \%$ confidence level. The GPS system is also susceptible to major service failures, in which the range accuracy exceeds 4.42 times the defined User Range Accuracy (URA) [6].

Different GPS augmentation systems have been developed to enhance GPS accuracy and integrity by compensating for the GPS errors. The Wide Area Augmentation System (WAAS) developed mainly for civil aviation navigation by Federal Aviation Administration (FAA) is a good example of these systems. The WAAS provides better accuracy and integrity than the GPS alone; however, more than 110 different technical failures have been reported since 2005 [7] besides the coverage limitation of this system [8]. 
Although critical for the development of autonomous flight control laws, a thorough investigation of the effects of GPS normal and abnormal operation on the performance of the control system has not yet been attempted. Therefore, a sensitivity analysis is performed in this research effort. A simplified GPS error model was developed and different accuracy parameters considered for the sensitivity study. Trajectory tracking control laws of different complexity were used to confirm the trend in the results. These control laws include fixed parameter control laws and adaptive control laws with increased fault tolerant capabilities.

\subsection{Research Objectives}

This thesis has the objective of developing a simplified GPS position and velocity error model, and using it to study the sensitivity of different UAV trajectory tracking controllers to GPS position and velocity error. The GPS error model was developed in Matlab/Simulink, with Graphical User Interface (GUI) and Matlab subsystem mask and integrated within the WVU UAV simulation environment. The error model statistically generates position and velocity errors, simulates the effect of GPS satellite configuration on the position measurement accuracy, and implements a set of failures to the GPS measurements. The controllers' performances were evaluated against the position and velocity time delay, update rate, error STD, biases, as well as failure conditions. The controllers' trajectory tracking performances were calculated using several metrics capturing performance in terms of tracking errors and control activity. Variable weights of different elements were considered depending on the local characteristics of the commanded trajectory. Detailed performance analysis and comparisons results are presented.

\subsection{Thesis Outline}

This thesis is organized in 8 chapters. After this brief introduction, Chapter 2 consists of a literature review mainly focused on development of GPS models and IGS product use. Chapter 3 introduces the Global Positioning System overview, its measurement error sources, some of its augmentation systems and its performance standard. Chapter 4 introduces the GPS error model, its mathematical expression, along with Simulink subsystem implementation and verification. Chapter 5 describes the WVU UAVs simulation environment, the controllers used in the analysis, as well as the controllers' trajectory tracking performance calculation algorithms. Chapter 6 
presents the controllers' performance sensitivity analysis results in the presences of different alterations of the GPS parameters. Chapter 7 consists of the conclusions of the comparison between the six controllers' performance. Chapter 8 is the bibliographical section. 


\section{Literature and Historic Review}

Within the literature review, three sections of previous work are discussed. These three sections are the modeling and simulation of the global positioning system, GPS failures, and the use of International GNSS Service's data and products.

\subsection{Modeling and Simulating the GPS}

The development of GPS error models for several purposes was the topic of many research papers. GPS and Differential GPS (DGPS) error modeling in a software format was introduced by Rankin [9]. The software package statically generates position error in a locally level coordinates and simulates the effect of the GPS satellite geometry on the position error. This software was used by NASA Langley Research Center for real time simulation purposes. Redmill used a slowly - time varying bias, a constant error bias, and Gaussian random noise generator to construct a GPS model for the evaluation and testing of on-vehicle sensors and control suites [10]. Perhinschi considered update rate, time delay, accuracy, and error dynamic to construct a GPS error model for small helicopter simulation [11]. Various contributors to GPS measurement errors were studied, modeled and then simulated to estimate their impact on the user segment performance in the aerospace navigation applications in [12]. Numerous GPS error model have been created based on the shaping filter approach in the frequency domain [13-15]. A model for GPS SPS receivers timing error using a multilayered neural network prediction was proposed by [16]. The GPS Signal In Space (SIS) error statistical characteristics for the recent three year were calculated by Heng [17].

Besides these GPS error models, several GPS simulators have been implemented. An Intermediate Frequency (IF) GPS signal simulator was implemented in Matlab by Dong, and a mathematical signal model was developed to express the IF GPS signal as a function of errors such as clock error, ionosphere errors, and troposphere error [18]. A GPS signal multipath simulator, which considers the effect of the signal reflection surrounding environment has been developed by Byun to estimate GPS multipath error in different environments [19]. A MATLAB toolbox has been developed by NAVSYS that allows modeling effects of interfering signal sources, 
antenna, and receiver characteristics on the received GPS signals [20]. A single frequency GPS receiver using the C/A code on the L1 carrier was implemented in Matlab Simulink [20].

The effects of scintillation on the availability and accuracy of GPS and Satellite Based Augmentation System (SBAS) was studied in [21]. The impact of the GPS receiver quality on the GPS position accuracy was studied in [22]. The effect of GPS receiver (station) position on the earth on the GPS positioning precision was studied by Wang [23]. Most of the GPS models implemented were designed mainly with the objective to reduce or eliminate the positioning error. In this approach the user does not have the direct control over the GPS position error. Even the GPS error models that were implemented for simulation purposes were limited by modeling the position errors only; furthermore, none of the models were aimed at of simulating the GPS failure conditions.

\subsection{GPS Failures}

GPS is a sophisticated communication system and different failures have been identified that can potentially affect the system. Ochieng presented a list of GPS failures that could occur on different levels from the data message production on GPS satellites until data reception and processing by the GPS receiver. He concluded the inadequacy of GPS to provide the required real time integrity information for different flight operations [24]. Bahtti summarized the stand alone GPS failure modes, the Inertial Navigation System (INS) failure modes, and the integrated GPS/INS failures modes on his research to improve integrated GPS/INS integrity [25]. The Failures Modes and Effect Analysis (FMEA) project run by Imperial College of London to capture and characterize GPS failure modes was overviewed by Minler. He developed a model of GPS failure occurrence for integrity monitoring algorithms design. The GPS failure modes, their probability of occurrence, and their effects were included in his summary [26]. The total failure of the GPS and its outage for 15 minutes on Dec 13, 2006 was studied by Afraimovich [27].

\subsection{The Use of International GNSS Service's (IGS) Data}

The IGS consists of more than 200 voluntary working agencies, universities, and research centers [28]. IGS collects, archives, and distributes GPS pseudorange and phase observations, broadcast

ephemerides and supporting types of raw data. IGS analyzes GPS raw data sets and uses them 
to form IGS products. IGS products support a wide range of scientific research such as: monitoring the troposphere and ionosphere, determination of satellites' orbits, and Earth rotation parameters [29]. The IGS data were used in this paper to study the GPS position characteristics.

\subsubsection{The gLAB Software}

gLAb is an advanced open source software package for GNSS data processing [30]. It was developed by a group of Astronomy and Geomatics (gAGE) researchers from the Technical University of Catalonia (UPC) for both educational and professional uses. The gLAB software is capable of processing both standard position service (SPS) and precise position service (PPS) with high accuracy; furthermore, the gLAB allows assessing every GPS source of error independently. gLAB features a graphical user interface (GUI) which allows the user easy uploading of the required files and setting of the analysis parameters. gLAB output data can be easily transferred to Matlab for further processing, thus it was used as a GPS data processor in this project. 


\section{The Global Positioning System}

The Global Positioning System (GPS) is a satellite based navigation system that consists of a group of 24 operational satellites, master control station, network of ground tracking stations, and users. The system continuously provides accurate position, velocity, and timing (PVT) information to an infinite number of users with appropriate receiving devices for any weather condition all over the world $[5,6]$. It was developed by the United States Department of Defense (DoD) originally for U.S. military use; however, it was made available to civil use as a secondary objective $[5,31]$. The system consists of three main segments: the space segment, the control segment, and the user segment [32].

\subsection{The Space Segment}

The GPS space segment (Figure 1) consists of a group of at least 24 operational satellites at 12,000 miles in altitude transmitting radio signals to users [5]. The 24 satellites are arranged in 6 orbital planes with 4 satellites per plane, and they are managed to ensure the availability of at least 4 GPS satellites for coverage of any point on the Earth [8]. Each satellite transmits two sine wave signals (carrier frequencies), two (or more) digital codes, and a navigation message. The receiver uses carriers and the codes to determine the distance to the GPS satellites while the navigation message contains the satellite coordinates (satellite position) as a function of time necessary to determine the receiver's position [33].

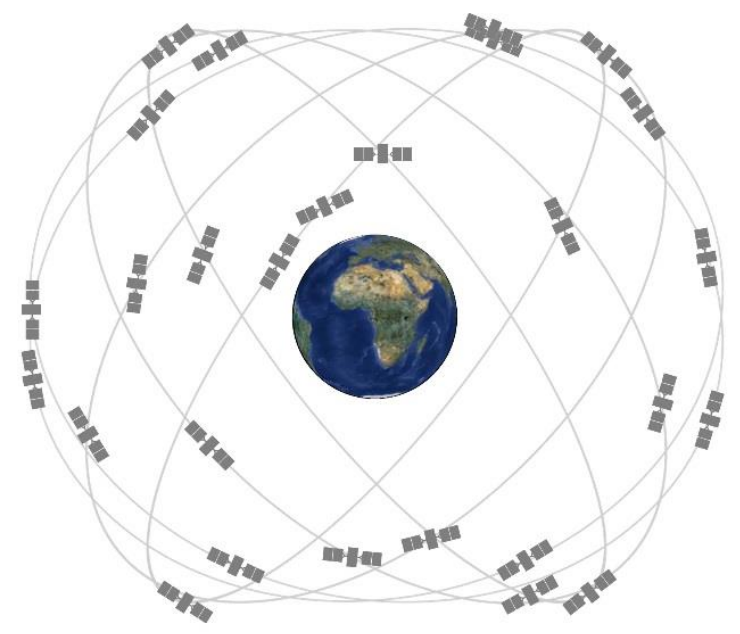

Figure 1: GPS Space Segment [8] 


\subsection{The Control Segment}

The GPS control segment consists of a network of ground stations. These stations include: the Master Control Station (MCS), an alternate master control station, global network of monitor stations, and ground antennas $[8,33]$. The purpose of the monitor stations is to collect GPS data and send that information to the Master Control Station for further processing. The Master Control Station performs further analysis on the GPS data collected to determine the GPS health and accuracy status. The MCS then generate a navigation message and update GPS satellites position data and sends this information to the space segment through antennas on the ground [5].

\subsection{The User Segment}

The third segment of the GPS is the user with an appropriate GPS receiver. The GPS provides two kinds of positioning services - one to the civil users and the second to the military (or DoD authorized) users. Civil users have access to the single frequency positioning service "Standard Positioning Service (SPS)", while the military users have the access to the dual frequency positioning service or the "Precise Positioning Service (PPS)" [6, 32].

\subsubsection{Standard Positioning Service (SPS).}

The GPS Standard Positioning Service (SPS) is defined in the GPS SPS performance standard document as "the positioning and timing service provided by way of ranging signals broadcast at the GPS L1 frequency. The L1 frequency, transmitted by all satellites, contains a coarse/acquisition (C/A) code ranging signal with a navigation data message that is available for peaceful civil, commercial, and scientific use" [6]. Before May 1, 2000 the accuracy of SPS was intentionally degraded by dithering the satellite's clock that introduces position error up to 100 meters through the Selective Availability (SA) policy; however, as of May 1, 2000 the SA would be turned off and currently remains off per United States government policy $[5,8]$. The performance standard of SPS signal in space (SIS) is defined by the United States government in the "Global Positioning Service Standard Positioning Service Performance Standard" document. 


\subsubsection{Precise Positioning Service (PPS)}

The GPS Precise Positioning Service (PPS) is defined in the GPS PPS performance standard document as "the positioning and timing service provided by way of authorized access to ranging signals broadcast at the GPS L1 and L2 frequencies. The L1 frequency, transmitted by all GPS satellites, contains a coarse/acquisition $(C / A)$ code ranging signal, and a precision $(P)$ code ranging signal with a navigation data message that is reserved for authorized use" [32]. The performance standard of the PPS signal in space (SIS) is defined by the United States government in the "Global Positioning Precise Standard Positioning Service Performance Standard" document.

\subsection{GPS Concept of Work}

The GPS concept of work is quite simple (Figure 2). It defines the user unknown position by using the user distance from at least four available satellites. Each satellite orbits the Earth continuously and transmits two frequencies: L1 at $1575.42 \mathrm{MHz}$ and L2 at $1227.60 \mathrm{MHz}$. The signals contain the information necessary to calculate the satellite precise position, the time the signal was sent, and information about satellite status. By using the difference between the time in which the signal was sent and its time of arrival (TOA) multiplied by the speed of light, the receiver will obtain the distance from each satellite. Therefore, by using the satellite position information, the receiver will have the capability to accurately determine its 3-dimensional position [5, 33].
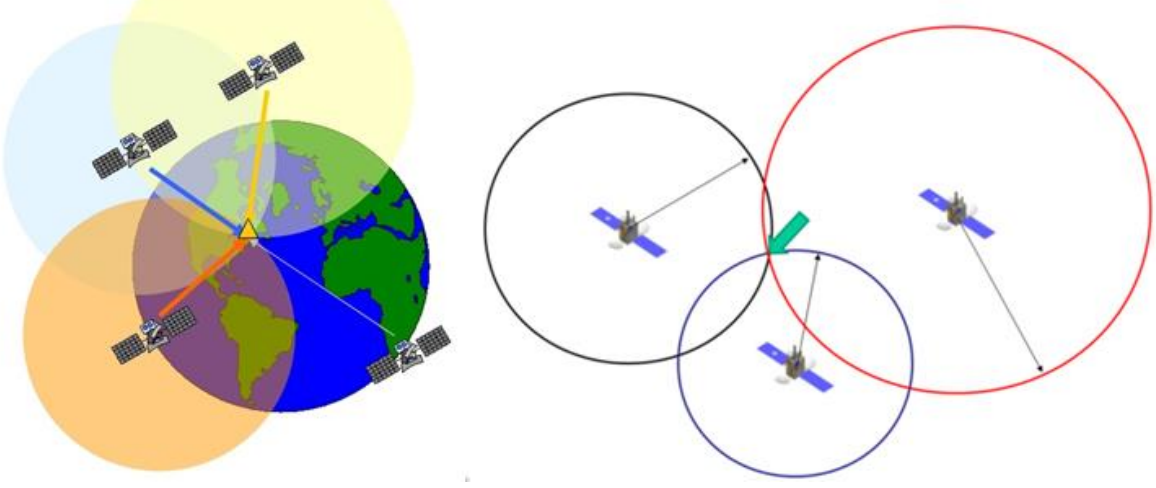

Figure 2: GPS Concept of Work [34, 35] 


\subsection{Performance of GPS}

The GPS system is subjected to some sources of error, just like any other communication system. Each source of error contributes somehow in the position, velocity, and time solution. Some of these errors can be corrected to some extent using some mathematical models; others need GPS receiver modification or external sensors to be corrected. Some sources of error will be briefly introduced next.

\subsubsection{Satellite Clock and Ephemeris Errors}

Each satellite has a highly stable atomic clock that controls all the timing operations [5]. However, these clocks are not perfect and their stability is approximately 1 to 2 parts in $10^{13}$ or better over a period of one day [33]. According to [36], satellite and receiver clock errors will produce three types of errors at the measurement level, which should be corrected. First, any clock error of $\delta$ t will cause a pseudo-range error of $c \delta t$, where ' $c$ ' is the speed of light. The second type of error produced by clock imperfection, consists of a computation error in the position of the satellite equal to $v_{s} \delta t$, where $v_{s}$ is velocity of the satellite. Finally, the third type of error affects the phase and is equal to $c \delta t / \lambda$, where $\lambda$ is GPS wave length.

Ephemeris errors are due to satellite position uncertainty. The satellites broadcast the information needed to calculate their precise position, but since the satellites are orbiting the Earth continuously and the modeling of the satellite movement is not ideal, the broadcasted information might not be accurate to precisely determine the satellite's actual position. The errors in the satellite's position lead to the ephemeris errors [5, 37]. The Ephemeris error might introduce a user position error of $1.6 \mathrm{~m}$ according to the International GNSS Service (IGS).

Master Control Station (MCS) monitors each satellite clock and position parameter to ensure the integrity of these parameters and periodically broadcast correction parameters to satellite that are included in the navigation message to keep the user position error within a specific range [36]. 


\subsubsection{Ionosphere Error}

One of the major sources of error in GPS measurement is the ionosphere effect. The ionosphere is located on the upper part of the atmosphere between $50 \mathrm{~km}$ and $1000 \mathrm{~km}$ or even higher above the Earth's surface $[5,33]$. In the ionosphere, the ultraviolet rays from the sun cause ionization of some of the gas molecules and free up some of their electrons. These free electrons will affect the GPS electromagnetic signal causing the carrier phase to advance the signal information (i.e., PRN code and navigation data) that will be delayed by the same amount of carrier phase advance $[5,37]$. This will affect the receiver-satellite measured distance, which will be shorter than the real distance if measured by the carrier phase, and longer if measured by the carrier code [33]. The ionospheric effect depends on the frequency of the passing-through signals. This property is very useful when using dual frequency (i.e., the PPS) in which the ionosphere effect can be measured and corrected to some extent [5].

\subsubsection{Tropospheric Effect}

The troposphere lies on the lower region of the atmosphere. It is electrically neutral, a nondispersive medium for the GPS signals [5, 33]. The troposphere delays both the GPS carrier phase and carrier code by the same amount, thus as a result the receiver- satellite measured distance will be longer than the real distance $[5,36]$. The amount of the delay in the troposphere is frequency independent; however, it depends on the temperature, pressure, humidity, and the zenith angle of sight to satellite [5]. The troposphere effect produces a receiver-satellite range error of the order of $2.4 \mathrm{~m}$ when the satellite is at the zenith (the satellite directly above the receiver). This error increases with the increase of the zenith angle to reach up to $28 \mathrm{~m}$ at $15^{\circ}$ elevation angle [33]. For this reason, it is always better to neglect satellites which have small elevation angles. As mentioned earlier, the troposphere effect is frequency-independent; therefore, using the dual frequency receiver (i.e., PPS) will not be useful to correct the troposphere effect; however, the troposphere effect can be calculate and corrected to some extend using mathematical models [33]. 


\subsubsection{Multipath Effect}

Multipath effect can potentially cause a considerable error in GPS measurement [5]. It occurs when a GPS signal arrives at the receiver's antenna through more than one path, primarily as the result of wave reflection by structures on the ground as shown in Figure $3[5,36]$. The multipath effect depends on the environment surrounding the receiver's antenna, as well as, the satellite movement relative to the receiver. The error caused by the multipath depends mainly on the receiver signal processing, the receiver antenna, and the reflected signal characteristics [5]. One of the simplest strategies to reduce the multipath effect is by installing a right-handed polarization antenna, which matches the GPS signal polarization. The reflection will change the signal polarization; therefore, the antenna will accept the direct GPS signal and reject the reflected signal [33].

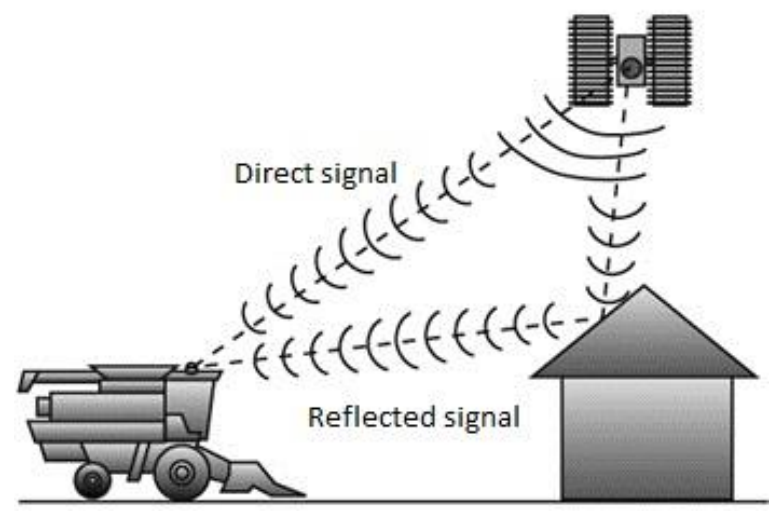

Figure 3: The Multipath Effect [38]

\subsubsection{Receiver Noise}

The receiver noise is introduced by the receiver's signal tracking loops. In general, the Delay Lock Loop (DLL) is used to track the code phase while the Phase Lock Loop (PLL) is used to track the carrier phase. The DLL introduces thermal noise and interference effects errors. The magnitude of DLL error is of the order of $10 \mathrm{~cm}(1 \sigma)$. While for the phase lock loop (PLL), the error is caused by the receiver noise and resolution. The magnitude of PLL error is of the order of $1.2 \mathrm{~mm}(1 \sigma)$ for $C / A$ code and $1.6 \mathrm{~mm}(1 \sigma)$ for $P(Y)$ code [5]. 


\subsubsection{Other Sources of Errors}

There are other sources of errors that affect the GPS accuracy. These effects should be considered and corrected especially when high accuracy is needed. These errors include but are not limited to:

Hardware delays: They include the satellites and user equipment time delays. Satellite time delay is the time difference between generating the signal and the time of its transmission. Receiver time delay is the difference in time between signal reception and its output to other user devices (e.g. controller, displays) due to signal processing by the GPS receiver [36].

Saganc Effect: It represents the error introduced because of the Earth rotation during the time the signal travels from the satellite to the receiver. The Earth rotation correction model should be applied or the Saganc effect might lead to a positioning error of the order of $30 \mathrm{~cm}$ [36].

Earth Tides: Earth tides are the displacement of the solid Earth body (i.e., the GPS station) due to the gravitation forces of the Sun and the Moon $[33,36]$. The Earth tides result in changes of the Earth's surface point location with respect to the axis of rotation [33]. The tide effect may reach a $60 \mathrm{~cm}$ displacement world-wide [5].

Table 1 summarizes the contribution of major sources of error to the User Equivalent Range Error (UERE) GPS measurement.

\begin{tabular}{|l|c|c|}
\cline { 2 - 3 } \multicolumn{1}{c|}{} & \multicolumn{2}{c|}{ UERE Contribution (1 $\sigma)(\mathrm{m})$} \\
\hline Error Source & SPS (Single Freq.) & PPS (Dual Freq.) \\
\hline Broadcast Clock & 1.1 & 1.1 \\
\hline Ephemeris Error & 0.8 & 0.8 \\
\hline lonosphere Error & 7.0 & 0.2 \\
\hline Tropospheric Effect & 0.2 & 0.2 \\
\hline Multipath Effect & 0.2 & 0.2 \\
\hline Receiver Noise & 0.1 & 0.1 \\
\hline Total UERE (RSS) & 7.1 & 1.4 \\
\hline
\end{tabular}

Table 1: Error Sources Contribution to GPS UERE Budget[5] 


\subsection{GPS Augmentation}

As of Dec 2012, the GPS SPS is expected to have less than $9 \mathrm{~m} 95 \%$ horizontal position accuracy and less than $15 \mathrm{~m}$ 95\% vertical position accuracy. In other words, the GPS SPS horizontal position error should be equal or smaller than $9 \mathrm{~m}$ for $95 \%$ of the time [6]. This accuracy might be good enough for some civil applications; however, it will not be sufficient for many civil applications such as aviation. For better GPS performance, GPS augmentation is required. According to [5] there are two different types of GPS augmentation: the differential GPS (DGPS) and external sensors aided GPS.

\subsubsection{Differential GPS (DGPS)}

The differential GPS ( Figure 4 ) is one of the methods used to improve GPS accuracy and integrity [37]. The idea behind the differential GPS is that some of the errors that cause GPS inaccuracy have similar effect on two receiver within a few hundreds of kilometers [33]. By setting one or more receivers at a precisely known position (i.e., station), this station will be able to calculate the biases of pseudorange of all seen satellites and then broadcast correction parameters to the in range compatible receivers (i.e., users) via radio frequencies [37]. According to [5], the correction parameters might include:

- Corrections to the user-satellites range measurements and corrections to GPS clock and ephemeris data.

- Pseudo-range and carrier phase measurements of the reference station.

- "Use" or "Do not use" for each seen satellite.

- Location, health, and meteorological information of the reference station. 


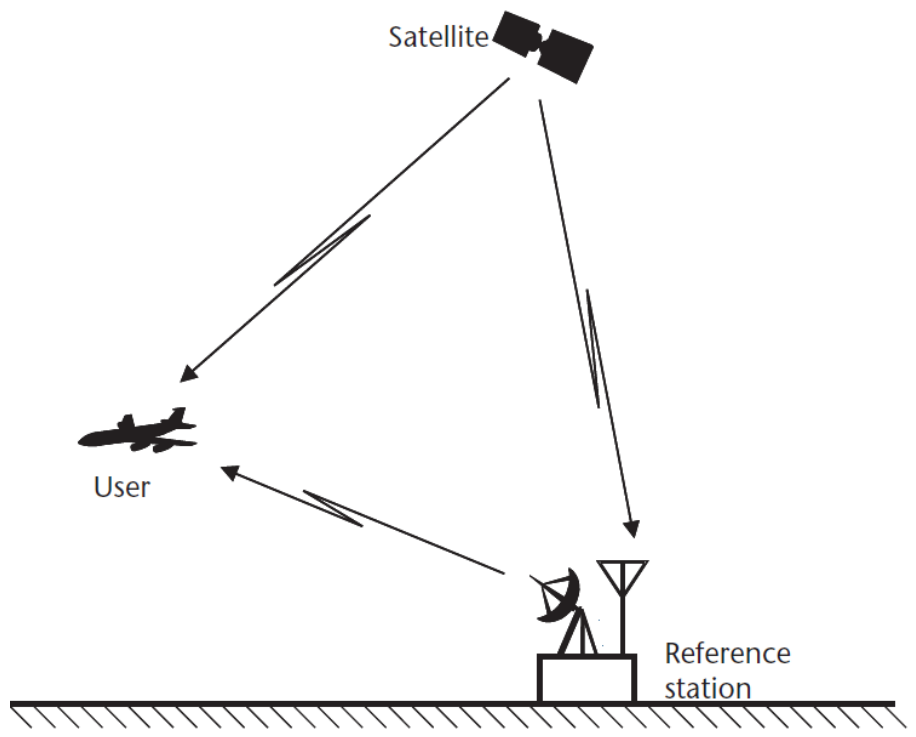

Figure 4: DGPS Concept of Work [5]

Public and private sectors have developed different GPS augmentation systems. These systems include but are not be limited to:

- Wide Area Augmentation System (WAAS). WAAS (Figure 5) is a satellite based augmentation system (SBAS). It was developed by the Federal Aviation Administration (FAA) mainly for civil aviation use; however, a recent study by FAA shows an increased use of this augmentation system in different civil applications. The WAAS covers the United States, Alaska, Hawaii, and parts of Puerto Rico, Mexico, and Canada. The WAAS structure consists of 38 Wide area Reference Station (WRS), Master Station (WMS), and Geostationary Commendation Satellites. The WRSs collect GPS data and then forward them to WAAS master station. The WMS collects and processes all the data received to determine the necessary corrections to be included in the augmentation signal. The augmentation message is then forwarded to a ground uplink station and will be broadcasted via the communication satellites. The WAAS was chosen as an example for differential GPS because of the wide-scale use of this system, as well as the availability of system performance and system failure technical reports. 


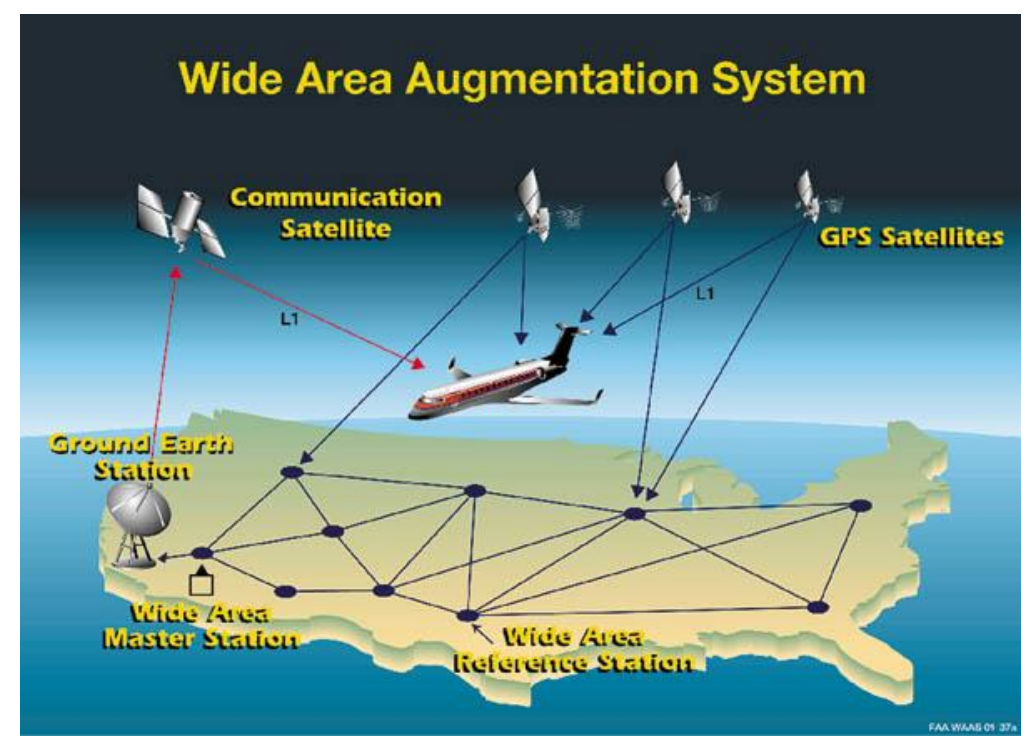

Figure 5: Wide Area Augmentation System [7]

- The Nationwide Differential GPS System (NDGPS). NDGPS is a ground based augmentation system (GBAS) that consists of two segments, the maritime segment which is operated by the United States Coast Guard, and the inland segment which is funded by the United States Department of Transportation. NDGPS provides accurate, highly reliable, real-time GPS correction signals to the users in coverage areas that include the entire Continental United States, the Great Lakes region, Puerto Rico, portions of Alaska, and Hawaii [39].

- The International GNSS Service (IGS). The IGS includes more than 200 voluntary working agencies that collaborate to generate precise GPS and other GNSS data and products. The IGS has a worldwide system that includes satellite tracking stations, data centers, and analysis centers. The IGS provides high quality GPS data and products that are used by scientists, engineers, and educators for a variety of purposes [28].

\subsection{GPS Position and Velocity at Normal and Abnormal Conditions}

In this section, the GPS normal and failure conditions will be defined based on:

- The GPS and WAAS performance standard documents.

- The GPS failure modes and WAAS technical reports. 
- The specification of GPS receivers available in the markets

\subsubsection{GPS Performance Standard}

The GPS SPS (or PPS) performance standard defined by the United States government is the level of SIS performance to be provided to the SPS (or PPS) users. The performance standards are defined by accuracy, availability, healthy, continuity, and Coordinated Universal Time (UTC) accuracy.

\subsubsection{SPS (or PPS) SIS Accuracy}

In the performance standard document, the SPS (or PPS) SIS position and velocity accuracy is defined by three aspects, all these four aspects are defined at $95 \%$ probability, and they are globally averaged by root mean square (rms) across part of the globe for enough period of time.

1) The User Rang Error (URE) (or the pseudorange accuracy).

2) The User Range Rate Error (URRE).

3) The User Range Acceleration Error (URAE).

The SPS (or PPS) accuracy standards do not include the effect of:

- Signal distortion caused by the atmosphere (i.e., ionosphere and troposphere) or any residual receiver delay compensation error.

- Receiver hardware or software noise and faults.

- Multipath and antenna errors or any user related errors.

For the purpose of this research, modified tables of the URE and URRE accuracy are presented; however, these two tables do not show all the information included in the original document. Age Of Data (AOD) in the tables refers to the time interval after the corrections have been sent to a satellite by the MCS. 


\begin{tabular}{|c|c|}
\hline \multicolumn{2}{|c|}{ URE SIS Accuracy Standard } \\
\hline SPS & PPS \\
\hline URE @ Normal Operation, Globally Averaged & URE @ Normal Operation, Globally Averaged \\
\hline Single-Frequency C/A Code & Dual-Frequency $\mathrm{P}(\mathrm{Y})$ Code \\
\hline 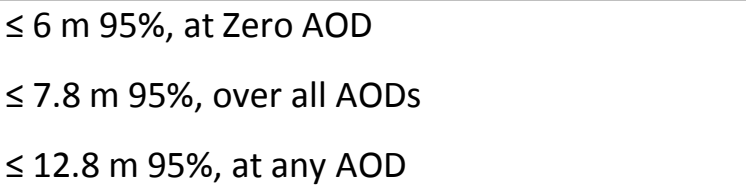 & 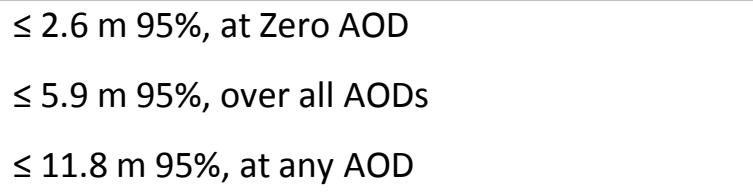 \\
\hline \multicolumn{2}{|l|}{$\begin{array}{l}\leq 30 \mathrm{~m} 99.94 \% \\
\leq 30 \mathrm{~m} 99.79 \% \text {, worst case single point }\end{array}$} \\
\hline URE @ Extended Operation & URE @ Extended Operation \\
\hline$\leq 388 \mathrm{~m}$, after 14 day without uploads & $\leq 388 \mathrm{~m}$, after 14 day without uploads \\
\hline
\end{tabular}

Table 2: URE SIS Accuracy Standard

\begin{tabular}{|c|c|}
\hline \multicolumn{2}{|c|}{ URRE SIS Accuracy Standard } \\
\hline SPS & PPS \\
\hline URRE @ Normal Operation, Globally Averaged & URRE @ Normal Operation, Globally Averaged \\
\hline Single-Frequency C/A Code & Dual-Frequency $\mathrm{P}(\mathrm{Y})$ Code \\
\hline $\begin{array}{l}\leq 0.006 \mathrm{~m} / \mathrm{s} 95 \% \text {, over any } 3 \mathrm{sec} \text { interval, any } \\
\text { AOD }\end{array}$ & $\begin{array}{l}\leq 0.006 \mathrm{~m} / \mathrm{s} 95 \% \text {, over any } 3 \text { sec interval, any } \\
\text { AOD }\end{array}$ \\
\hline
\end{tabular}

Table 3: URRE SIS Accuracy Standard

\subsubsection{SPS (or PPS) SIS Integrity}

The SPS (or PPS) integrity is the level of confidence that can be placed in the accuracy of the information provided by the GPS signal. The GPS service integrity is defined by four aspects:

- SIS URE Not-to-Exceed (NTA) tolerance represents, the boundaries of URE for which the satellite is considered to be "healthy".

- $\quad$ The probability of major service failure represents the probability that a satellite SIS URE will exceed the SIS URE NTA value without an alert being issued.

- Time to Alert (TTA) is the time from the failure occurrence until an alert is being issued and received by the GPS receiver antenna. 
- Alert-Alarm Indications are expected to ensure maximum SIS integrity. A set of alert and alarm will be indicated and included in the navigation message. Nine different alert will be indicated when the SIS might carry incorrect information, while an alert will be indicated when SIS has a MSI (Miss Leading Information).

\subsubsection{Other SPS (or PPS) Performance Characteristics}

There are other SPS (or PPS) performance characteristics specified in the performance documents. Below is a brief definition of each one of them.

- SPS (or PPS) SIS Availability: the probability that the slots in GPS constellation, (i.e., the 24 slots), will have satellites broadcasting a trackable and healthy SPS (or PPS) signal. It is either expressed by per slot availability or by the constellation availability. Per slot availability is the portion of time that a slot in GPS constellation will have a satellite broadcasting trackable and healthy signals, while constellation availability is the portion of time a known number of slots in GPS constellation will have satellites that are broadcasting trackable and healthy signal [6, 32].

- SPS (or PPS) SIS Health: each GPS satellite broadcast real-time health status as an integral part of the SPS (or PPS) SIS. The satellite status can take one value of the three healthy, marginal, or unhealthy. The satellite status depends on information being broadcasted by that satellite. The healthy satellite is broadcasting information within the allowed operation limits. The marginal satellite might be broadcasting inaccurate information, while the unhealthy satellite is broadcasting information outside the allowed range [6, 32].

- SPS (or PPS) SIS Continuity: the probability that, over an hour time duration, the SIS will continue to provide accurate information to the users without any unscheduled interruption [6, 32].

- SPS (or PPS) SIS UTC Accuracy: the accuracy of SPS (or PPS) SIS information that relates GPS time to UTC. 


\subsubsection{Position, Velocity, and Time (PVT) Accuracy Estimation}

In the GPS SPS (or PPS) performance document, the position accuracy is expressed in the term of pseudorange error (i.e. URE), and the velocity accuracy in term of pseudorange rate error (i.e. URRE); furthermore, these accuracy parameters exclude any error outside the control of GPS space or control segments. To calculate the final receiver position, velocity, and time accuracy, the excluded error should be included and the effect of the range error on the final position and velocity solution should be studied.

\subsubsection{User Equivalent Range Error (UERE)}

According to [5], the User Equivalent Range Error (UERE) is the statistical summation of contribution of space, control, and user segment errors. The contribution of space and control segments to UERE error budget was defined by the URE. The contribution of the user segment is defined by the User Equivalent Error (UEE). The UEE error budget includes the contribution of atmosphere effect, multipath effect, and any receiver related errors. The UEE budget differs from one receiver to another. Table 4 shows the UEE budget for different receivers. The ionospheric delay compensation does not apply to a single frequency receiver so it does not appear in the table.

\begin{tabular}{|c|c|c|c|c|}
\hline Error Source & $\begin{array}{c}\text { Traditional } \\
\text { Receiver (single } \\
\text { Freq.) }\end{array}$ & $\begin{array}{c}\text { Improved } \\
\text { Receiver } \\
\text { (single Freq.) }\end{array}$ & $\begin{array}{c}\text { Modern } \\
\text { Receiver } \\
\text { (Single Freq.) }\end{array}$ & $\begin{array}{c}\text { Dual } \\
\text { Frequency } \\
\text { Receiver }\end{array}$ \\
\hline $\begin{array}{c}\text { lonospheric Delay } \\
\text { Compensation }\end{array}$ & N/A & N/A & N/A & 0.8 \\
\hline $\begin{array}{c}\text { Tropospheric Delay } \\
\text { Compensation }\end{array}$ & 3.9 & 4.0 & 3.9 & 1.0 \\
\hline Receiver Errors & 2.9 & 2.0 & 2.0 & 4.0 \\
\hline Multipath & 2.4 & 0.5 & 0.2 & 0.2 \\
\hline Other Users Segment error & 1.0 & 1.0 & 1.0 & 0.8 \\
\hline UEE (m) 95\% & 5.5 & 4.6 & 4.5 & 1.6 \\
\hline
\end{tabular}

Table 4: UEE Error Budget in meters [6] 
Equation (1) is used to calculate the UERE from the appropriate GPS SPS (or PPS) SIS URE value with the UEE for the GPS receiver used.

$$
U E R E=\sqrt{U R E^{2}+U E E^{2}}
$$

\subsubsection{Dilution of Precision (DOP)}

The Dilution of Precision (DOP) is a geometric factor used to express the effect of GPS satellites geometry seen by a receiver on the precision of the receiver measurements [33]. The easiest way to understand the effect of DOP on the precision of a GPS receiver measurement is by using the geometry approach. Figure 6 illustrates the effect of DOP on the GPS receiver measurement. The black circles represent the real distance between point $A$ and the satellites, while the gray area represents the URER for each satellite. In Figure 6-a, the two satellites have good geometry configuration relative to point $A$, whose measured position lies in the small blue area. In Figure 6-b, the satellites have poor geometry configuration with respect to point $A$, whose measured position now lies in a larger blue area, thus affected by a larger error.

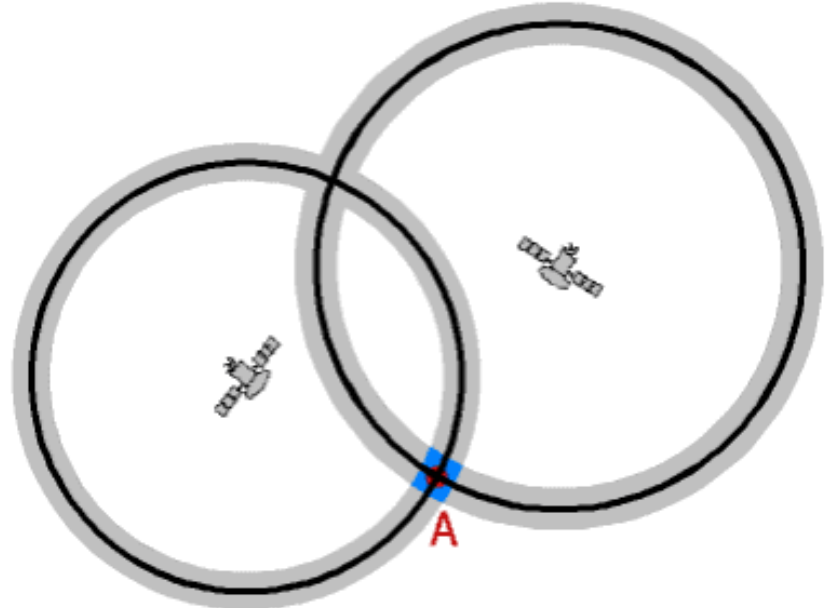

(a)

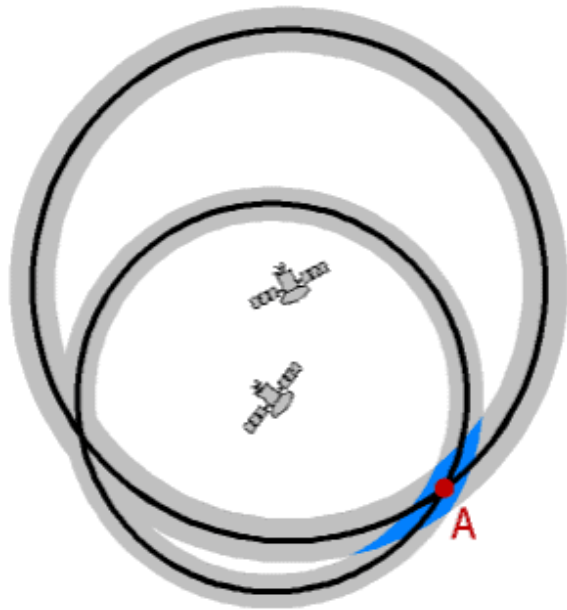

(b)

Figure 6: DOP Effect [40]

There are several DOP parameters used to calculate position, velocity, or time accuracy. These parameters are the Geometric Dilution of Precision (GDOP), Position Dilution of Precision (PDOP), Horizontal Dilution of Precision (HDOP), Vertical Dilution of Precision (HDOP), and Time Dilution 
of Precision (HDOP) [5, 33]. Equations 2 and 3 show the relationships between these different parameters.

$$
\begin{aligned}
& G D O P=\sqrt{P D O P^{2}+T D O P^{2}} \\
& P D O P=\sqrt{H D O P^{2}+V D O P^{2}}
\end{aligned}
$$

\subsubsection{The Basic Equations of PVT Accuracy}

As seen before, each and every segment of the GPS has introduced errors in the GPS pseudorange measurements (i.e. UERE). These errors will affect the position, velocity, and time measurement accuracy in a complicated way [5]; however, equation (4) has been found to be sufficient for many applications $[5,6,33]$ to calculate the effect of pseudorange, pseudorange rate, and time UTC accuracy on the finial receiver position, velocity, and time solution.

GPS Solution Accuracy $=$ An Error Factor $*$ Corresponding DOP factor

Using equation (4), the following accuracy variables can be derived:

$$
\begin{aligned}
& \text { User Horizontal Navigation Error }(U H N E)=U E R E * H D O P \\
& \text { User Vertical Navigation Error }(U V N E)=U E R E * V D O P \\
& \text { User Horizontal Velocity Error }=U E R R E * H D O P \\
& \text { User Vertical Velocity Error }=U E R R E * V D O P
\end{aligned}
$$

\subsection{WAAS Performance Standard}

The WAAS performance standard document follows the structure of the GPS performance documents; however, the WAAS accuracy is given in terms of user end performance. Table 5 summarizes the WAAS minimum performance parameters. 


\begin{tabular}{|l|l|}
\hline Integrity & Performance \\
\hline Time To Alert & $8 \mathrm{sec}$ \\
\hline Continuity & $1-8^{*} 10^{-7} / 15 \mathrm{sec}$ \\
\hline Horizontal Accuracy Nominal & $1.6 \mathrm{~m}(95 \%)$ \\
\hline Horizontal Accuracy Maximum & $12 \mathrm{~m}$ (maximum observed) \\
\hline Horizontal Accuracy Limit & $4 \mathrm{~m}$ (conservative 95\%) \\
\hline Vertical Accuracy Nominal & $1.6 \mathrm{~m}(95 \%)$ \\
\hline Vertical Accuracy Maximum & $12 \mathrm{~m}$ (maximum observed) \\
\hline Vertical Accuracy Limit & $4 \mathrm{~m}$ (conservative 95\%) \\
\hline
\end{tabular}

Table 5: WAAS Performance [8]

\subsection{GPS Integrity Failures}

GPS accuracy is monitored by the control segments to ensure that SPS (or PPS) SIS URE are within the limits of SPS (or PPS) SIS URE NET [6, 32]; however, failures might occur causing that SPS (or PPS) SIS exceed the SPS (or PPS) URE limits [26]. Failures when the SPS (or PPS) SIS continues to be healthy, but the URE exceeds the URE NET are called "soft" failures, while the "hard" failures might occur when the SPS (or PPS) is not trackable anymore [6]. The probability of failures occurrence is very low $\left(10^{-4}\right.$ per hour for stand-alone GPS) and some of these failures are detectable by the Receiver Autonomous Integrity Monitoring (RAIM) [6, 26]. Below is a modified version of a table summarized by [26], which contains the failures modes that might introduce an integrity risk because they might not be detectable by satellite or receiver faults detecting algorithms. Table 6 contains the failure modes, their probability of occurrence, along with their effects. 


\begin{tabular}{|c|c|c|c|}
\hline Failure Mode & Probability of Occurrence & Type & Magnitude \\
\hline Clock Jump & 1.0e-1/SV/year & Step & $0-30 \mathrm{~m}$ \\
\hline Clock Frequency Jump/Drift & 1.0e-1/SV/year & Ramp & $2.5 \mathrm{~m} / \mathrm{sec}$ \\
\hline $\begin{array}{l}\text { Clock Drift/Phase-Runoff } \\
\text { (Atomic Clock Power Supply) }\end{array}$ & 6.6e-3/SV/year & Ramp & $54 \mathrm{~km} / \mathrm{hr}$ \\
\hline $\begin{array}{l}\text { Clock Drift/Phase-Runoff } \\
\text { (Atomic Clock Electronics) }\end{array}$ & 1.1e-2/SV/year & Ramp & $10 \mathrm{~m} / \mathrm{hr}$ \\
\hline $\begin{array}{l}\text { Clock Drift/Phase-Runoff } \\
\text { (Atomic Clock Servo Mechanism) }\end{array}$ & 1.0e-2/SV/year & Ramp & $10 \mathrm{~m} / \mathrm{hr}$. \\
\hline $\begin{array}{l}\text { Clock Drift/Phase-Runoff } \\
\text { (Atomic Clock CS/Rb tube) }\end{array}$ & 3.3e-1/SV/year & Ramp & $10 \mathrm{~m} / \mathrm{hr}$. \\
\hline $\begin{array}{c}\text { Clock Drift/Phase-Runoff } \\
\text { (Atomic Clock Tuning Register) }\end{array}$ & 2.1e-5/SV/year & Ramp & $2164 \mathrm{~m} / \mathrm{hr}$. \\
\hline Clock Frequency Jump & 1.0/SV/year & Ramp & $2.0 \mathrm{~m} / \mathrm{hr}$. \\
\hline FSDU Upsets & 3.0e-1/SV/year & Step & $0-30 \mathrm{~m}$ \\
\hline Meteor Impact (Delta_V) & TBD & Ramp & $0-10 \mathrm{~m} / \mathrm{hr}$. \\
\hline $\begin{array}{c}\text { MCS Upload Error } \\
\text { (Bad Earth Orientation Data) }\end{array}$ & 5.2e-8/const/year & Ramp & $.00056 \mathrm{~m} / \mathrm{s}$ \\
\hline $\begin{array}{c}\text { MCS Upload Error } \\
\text { (Single Freq. Ion. Model) }\end{array}$ & 2.4e-2/upload & Noise & $13.7 \mathrm{~m}(1 \sigma)$ \\
\hline $\begin{array}{c}\text { Operational Error } \\
\text { (Too early return to 'healthy 'status) }\end{array}$ & 5.0e-5/const/year & Step: Ramp & $120 \mathrm{~m}: 5 \mathrm{~m} / \mathrm{hr}$. \\
\hline $\begin{array}{l}\text { Operational Error } \\
\text { (Not flagged 'unhealthy') }\end{array}$ & 5.0e-5/const/year & Ramp & $120 \mathrm{~m} / \mathrm{hr}$. \\
\hline $\begin{array}{l}\text { Operational Error } \\
\text { (Incorrect database Control element) }\end{array}$ & 6.2e-9/const/year & Ramp & $0.0-0.3 \mathrm{~m} / \mathrm{sec}$ \\
\hline
\end{tabular}

*SV refer to Single Vehicle

*const refer to the GPS consolation

Table 6: GPS Failure Modes [26] 
The GPS receiver velocity reading accuracy is a function of URRE (see equation 7, and 8 ), and since the URRE is a function of the URE; therefore, the failures in the URE (i.e., position failures) will always lead to failures in the URRE (i.e., velocity failure) [6]. Figure 7 shows the URE and URRE failures type.

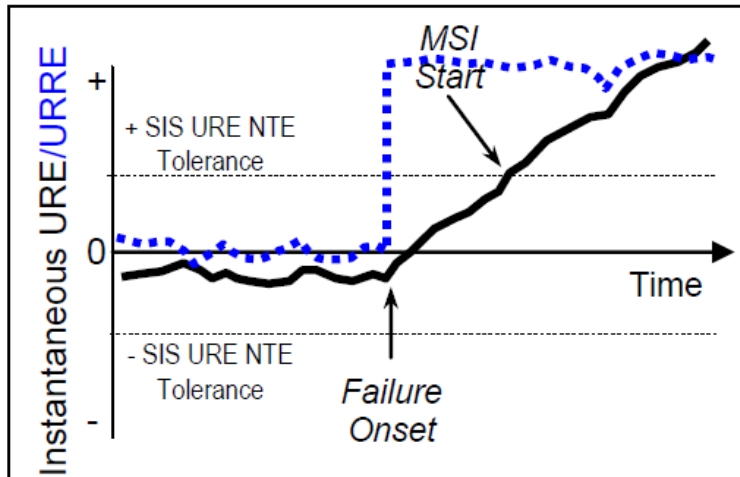

a. URE Ramp Error - URRE Step Error

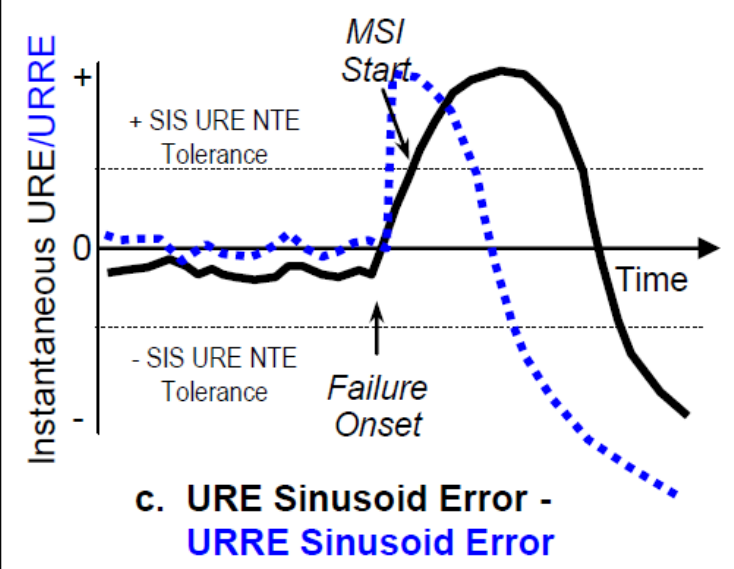

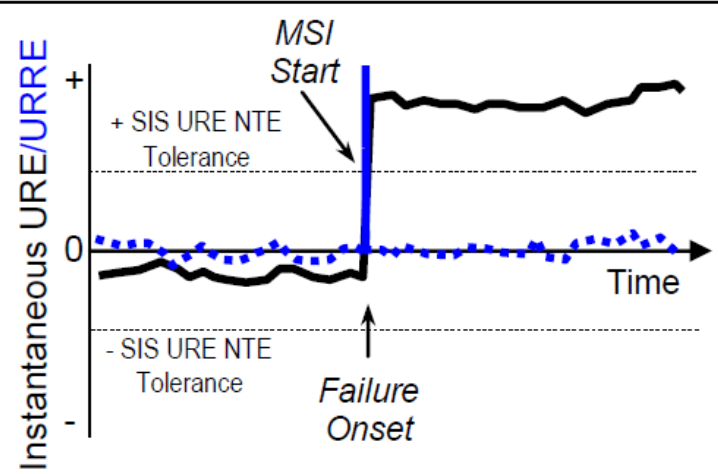

b. URE Step Error - URRE Spike

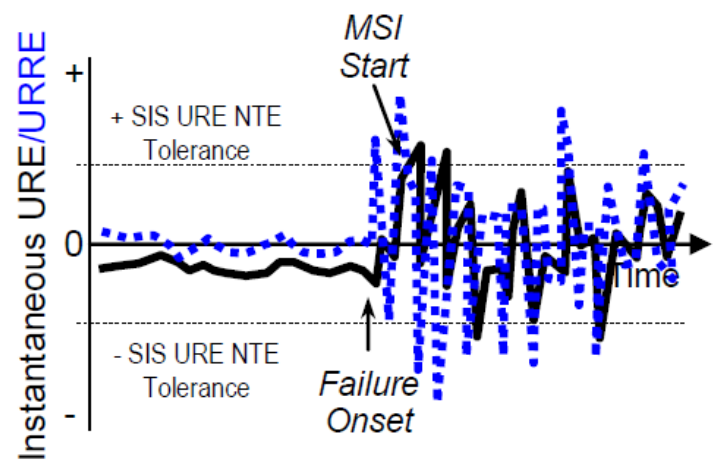

d. URE Noise Error - URRE Noise Error

Figure 7: Types of GPS URE/URRE Failures [6]

\subsection{WAAS Failures}

The WAAS was mainly designed to correct for GPS errors and enhance overall GPS performance; however, the Federal Aviation Administration (FAA) has reported many failures that affected the performance of the WAAS. These failures along with the causes and their effects on the WAAS are available online at [7]. A brief description of the most common WAAS failures, the causes, and their effects are listed below. 
- WAAS SIS outage: The SIS outage means the loss of SIS transmission from the WAAS geostationary satellites. The SIS outage may occur in single vehicle or multiple vehicles at the same time. It is typically caused by major software failures, equipment failures, or storms. The SIS outages have been recorded to last for a period between $110 \mathrm{sec}$ and $2096 \mathrm{sec}$.

- Loss of availability: The loss of availability refers to the failures in the GPS system that affect the WAAS capability to calculate and send the correction parameters to the users. Such failures include unscheduled GPS satellites maintenance, too many unhealthy GPS satellites at the same time, or very poor GPS satellite geometry.

- Loss of service: The FAA formulates the required WAAS performance specifically for each phase of flight operation. These performance requirements are presented in Table 7. They are subdivided into En-route, Terminal, LNAV (Lateral Navigation), LNAV/VNAV (Lateral/Vertical Navigation), LPV (Localizer Performance with Vertical guidance), and LPV-200 flight operations. The loss of service indicates that the WAAS is no more able to satisfy one or more integrity requirement of that category. WAAS loss of service has been recorded to be caused by ionospheric storms, radio frequency interference, software failure, or minor hardware failure. 


\begin{tabular}{|c|c|c|c|c|c|c|}
\hline & EnRoute & Terminal & LNAV & $\begin{array}{l}\text { LNAV } \\
\text { /VNAV }\end{array}$ & LPV & LPV200 \\
\hline TTA & $15 \mathrm{~s}$ & $15 s$ & $10 \mathrm{~s}$ & $10 \mathrm{~s}$ & $6.2 \mathrm{~s}$ & $6.2 \mathrm{~s}$ \\
\hline HAL & $2 \mathrm{~nm}$ & $1 \mathrm{~nm}$ & $556 \mathrm{~m}$ & $556 \mathrm{~m}$ & $40 m$ & $40 m$ \\
\hline VAL & $\mathrm{N} / \mathrm{A}$ & N/A & $\mathrm{N} / \mathrm{A}$ & $50 \mathrm{~m}$ & $50 \mathrm{~m}$ & $35 \mathrm{~m}$ \\
\hline Probability of HMI & $\begin{array}{l}10^{-7} \\
\text { per hour }\end{array}$ & $\begin{array}{c}10^{-7} \\
\text { per hour }\end{array}$ & $\begin{array}{c}10^{-7} \\
\text { per hour }\end{array}$ & $\begin{array}{l}2 \times 10^{-7} \text { per } \\
\text { approach }\end{array}$ & $\begin{array}{c}2 \times 10^{-7} \text { per } \\
\text { approach } \\
\text { (150 seconds) }\end{array}$ & $\begin{array}{c}2 \times 10^{-7} \text { per } \\
\text { approach } \\
\text { (150 seconds) }\end{array}$ \\
\hline Zone 1 Continuity & $\begin{array}{l}1-10^{-5} \\
\text { per hour }\end{array}$ & $\begin{array}{c}1-10^{-5} \\
\text { per hour }\end{array}$ & $\begin{array}{l}1-10^{-5} \\
\text { per hour }\end{array}$ & $\begin{array}{l}1-5.5 \times 10^{5} \\
/ 15 \text { seconds }\end{array}$ & $\begin{array}{l}1-8 \times 10^{-6} \\
/ 15 \text { seconds }\end{array}$ & $\begin{array}{l}1-8 \times 10^{-6} \\
/ 15 \text { seconds }\end{array}$ \\
\hline $\begin{array}{c}\text { Horizontal } \\
\text { Accuracy } \\
(95 \%)\end{array}$ & $0.4 \mathrm{~nm}$ & $0.4 \mathrm{~nm}$ & $220 m$ & $220 m$ & $16 \mathrm{~m}$ & $16 m$ \\
\hline $\begin{array}{c}\text { Vertical Accuracy } \\
(95 \%)\end{array}$ & N/A & $\mathrm{N} / \mathrm{A}$ & $\mathrm{N} / \mathrm{A}$ & $20 m$ & $20 m$ & $4 m$ \\
\hline $\begin{array}{c}\text { Availability } \\
\text { (Zone } 1 \text { Coverage) }\end{array}$ & $\begin{array}{c}0.99999 \\
(100 \%)\end{array}$ & $\begin{array}{c}0.99999 \\
(100 \%)\end{array}$ & $\begin{array}{c}0.99999 \\
(100 \%)\end{array}$ & $\begin{array}{c}0.99 \\
(100 \%)\end{array}$ & $\begin{array}{c}0.99 \\
(80-100 \%)\end{array}$ & $\begin{array}{c}0.99 \\
(40-60 \%)\end{array}$ \\
\hline $\begin{array}{c}\text { Availability } \\
\text { (Zone } 2 \text { Coverage) }\end{array}$ & $\begin{array}{c}0.999 \\
(100 \%)\end{array}$ & $\begin{array}{c}.999 \\
(100 \%)\end{array}$ & $\begin{array}{c}.999 \\
(100 \%)\end{array}$ & $\begin{array}{c}.95 \\
(75 \%)\end{array}$ & $\begin{array}{c}0.95 \\
(75 \%)\end{array}$ & $\mathrm{N} / \mathrm{A}$ \\
\hline $\begin{array}{c}\text { Availability } \\
\text { (Zone } 3 \text { Coverage) }\end{array}$ & $\begin{array}{c}0.999 \\
(100 \%)\end{array}$ & $\begin{array}{c}.999 \\
(100 \%)\end{array}$ & $\begin{array}{c}.999 \\
(100 \%)\end{array}$ & $\mathrm{N} / \mathrm{A}$ & $\mathrm{N} / \mathrm{A}$ & N/A \\
\hline $\begin{array}{c}\text { Availability } \\
\text { (Zone } 4 \text { Coverage) }\end{array}$ & $\begin{array}{c}0.999 \\
(100 \%)\end{array}$ & $\begin{array}{c}.999 \\
(100 \%)\end{array}$ & $\begin{array}{c}.999 \\
(100 \%)\end{array}$ & $\mathrm{N} / \mathrm{A}$ & $\mathrm{N} / \mathrm{A}$ & $\mathrm{N} / \mathrm{A}$ \\
\hline $\begin{array}{c}\text { Availability } \\
\text { (Zone } 5 \text { Coverage) }\end{array}$ & $\begin{array}{c}0.99999 \\
(100 \%)\end{array}$ & $\begin{array}{c}.999 \\
(100 \%)\end{array}$ & $\begin{array}{c}.999 \\
(100 \%)\end{array}$ & $\mathrm{N} / \mathrm{A}$ & $\mathrm{N} / \mathrm{A}$ & N/A \\
\hline
\end{tabular}

Table 7: WAAS Navigation Performance Requirements 


\section{GPS Error Model}

\subsection{General Model Architecture}

To analyze the effect of GPS position and velocity errors on autonomous flight control systems, a GPS error model is needed to transform the actual position and velocity produced by the simulation model into measured position and velocity values. The GPS error model should also be capable of generating various GPS position and velocity error scenarios to cover all GPS normal and abnormal operations of interest.

Let the actual position vector of the center of mass of the aircraft $A$ with respect to the origin 0 of the Earth Centered, Earth Fixed (ECEF) fixed reference frame E be $\vec{r}_{a}^{O A}$. The components of the actual position vector with respect to the Earth axes are denoted as:

$$
\left[\vec{r}_{a}^{O A}\right]_{E}=\left[\begin{array}{c}
X_{a} \\
Y_{a} \\
Z_{a}
\end{array}\right]_{E}
$$

In a similar manner, the measured components of the position vector are expressed as:

$$
\left[\vec{r}_{m}^{O A}\right]_{E}=\left[\begin{array}{c}
X_{m} \\
Y_{m} \\
Z_{m}
\end{array}\right]_{E}
$$

Let the position error components generated by the GPS error model be denoted as:

$$
[\overrightarrow{p e}]_{E}=\left[\begin{array}{c}
p e_{x} \\
p e_{y} \\
p e_{z}
\end{array}\right]_{E}
$$

then

$$
\left[\vec{r}_{m}^{O A}\right]_{E}=\left[\vec{r}_{a}^{O A}\right]_{E}+[\overrightarrow{p e}]_{E}
$$

In a similar manner, let the actual velocity vector of the center of mass of the aircraft A with respect to the origin $\mathrm{O}$ of the Earth-fixed reference frame $\mathrm{E}$ be represented by ${ }^{O} \vec{v}_{a}^{A}$. The components of the actual velocity vector with respect to the Earth axes are denoted as: 


$$
\left[{ }^{O} \vec{v}_{a}^{A}\right]_{E}=\left[\begin{array}{c}
\dot{X}_{a} \\
\dot{Y}_{a} \\
\dot{Z}_{a}
\end{array}\right]_{E}
$$

and the measured components of the velocity vector are expressed as

$$
\left[{ }^{O} \vec{v}_{m}^{A}\right]_{E}=\left[\begin{array}{c}
\dot{X}_{m} \\
\dot{Y}_{m} \\
\dot{Z}_{m}
\end{array}\right]_{E}
$$

Let the velocity error components generated by the GPS error model be denoted as:

$$
[\overrightarrow{v e}]_{E}=\left[\begin{array}{c}
v e_{x} \\
v e_{y} \\
v e_{z}
\end{array}\right]_{E}
$$

Then

$$
\left[{ }^{O} \vec{v}_{m}^{A}\right]_{E}=\left[{ }^{O} \vec{v}_{a}^{A}\right]_{E}+[\overrightarrow{v e}]
$$

Figure 8 illustrates the general block diagram of the GPS position error model. The GPS error model parameter will specify the position error characteristics that will be added to the actual position data.

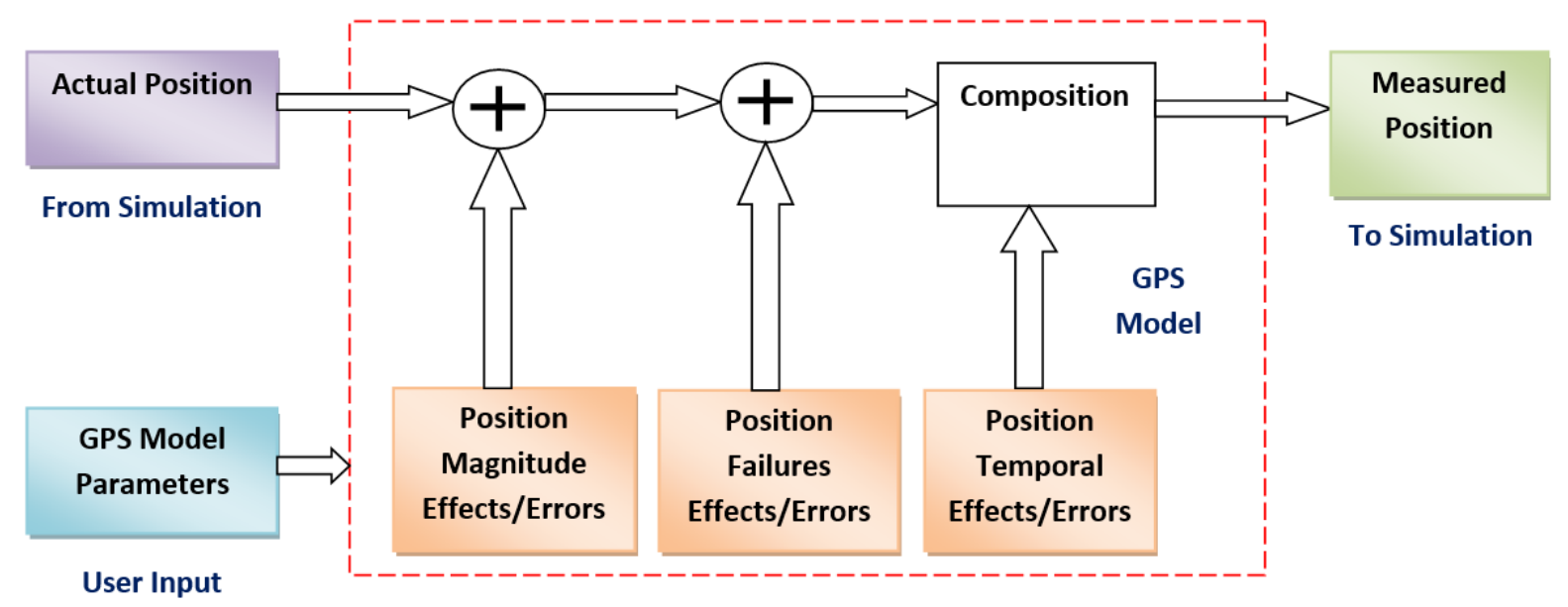

Figure 8: GPS Position Error Model Block Diagram

The proposed GPS velocity error model (see Figure 9) depends on the GPS position error model because the GPS position accuracy will affect the velocity accuracy to a certain degree depending on the algorithm used to calculate the velocity. 


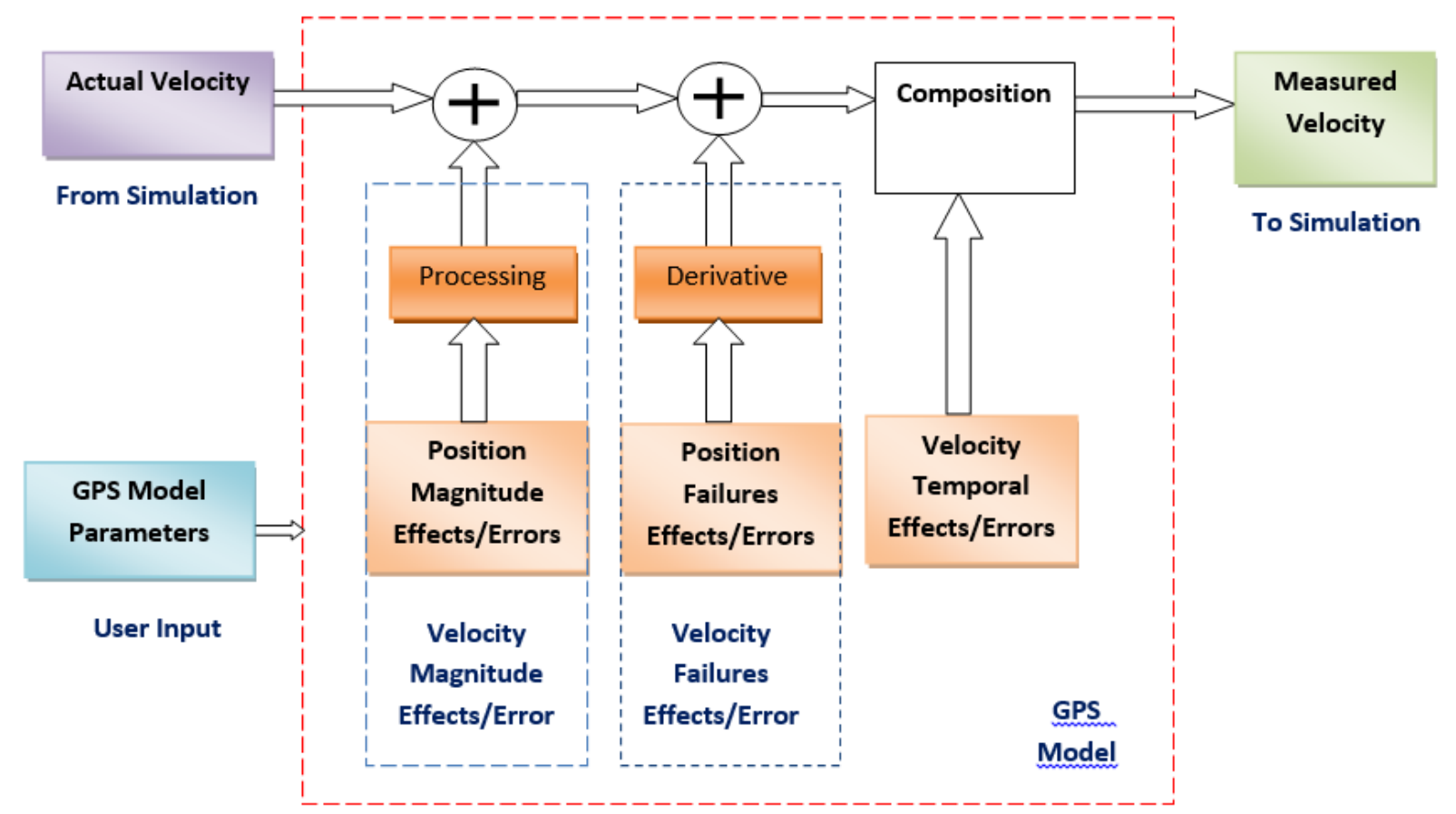

Figure 9: GPS Velocity Error Model Block Diagram

Equations (5) and (6) from the basic equation of PVT accuracy provide an accuracy estimation of the GPS position error based on the UERE and the DOP, thus the position error will be:

$$
[\overrightarrow{p e}]_{E}=\left[\begin{array}{l}
U E R E(t) * \operatorname{HDOP}(t) \\
\operatorname{UERE}(t) * \operatorname{HDOP}(t) \\
\operatorname{UERE}(t) * \operatorname{VDOP}(t)
\end{array}\right]
$$

However, for the purpose of increasing model flexibility, the UERE error will be considered to be independent on each axis. Furthermore, biases and other abnormal output variations, referred to as failures should be considered in order to simulate a wide range of GPS normal and abnormal conditions. Therefore, the mathematical expression of the GPS position error will be:

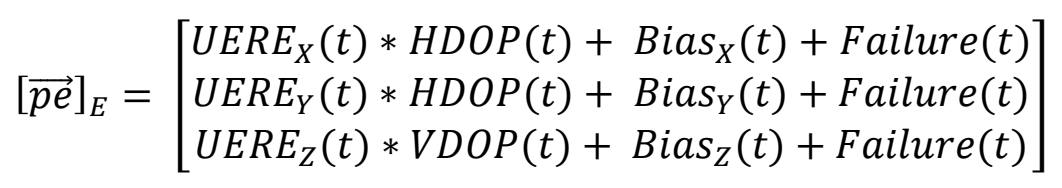

Different approaches are commonly used to measure the GPS receiver velocity. The typical approach for providing velocity measurements is by using the derivative of position data points [5]. In other words, if the receiver's position at time $t 1$ was $p(t 1)$, and the receiver's position at time $t 2$ was $p(t 2)$, then the receiver's velocity $V$ would be: 


$$
V=\frac{p(t 2)-p(t 1)}{t 2-t 1}
$$

This approach provides a "good" velocity estimate for a receiver moving at a constant speed; however, small position errors will lead to a large velocity error, if the (t2-t1) value is relatively small. A modified approach is to use a larger time interval to minimize the position error effect; however, this will not eliminate the position error effect on the velocity.

A better velocity measurement accuracy was achieved by using the Doppler shift. The Doppler shift is the change of a wave frequency due to the relative movement of the source and the receiver. Different algorithms are using the Doppler shift phenomenon to determine the receiver velocity, and high accuracy GPS velocity measurements can be achieved.

Whether using the position track point or the Doppler approach, the receiver position accuracy will affect the calculation of the receiver velocity $[5,6]$ and since the velocity accuracy follows the basic equation of PVT accuracy equation, an assumption has been made to calculate the velocity error as a scaled version of the corresponding position error. Furthermore, the position failure will appear as a time derivative on the velocity error (see Figure 7). In other words, the velocity error components will be:

$$
[\overrightarrow{v e(t)}]_{E}=\left[\begin{array}{l}
k * U E R E_{X}(t) * \operatorname{HDOP}(t)+\frac{d}{d t}(\text { Failure }(t)) \\
k * U E R E_{Y}(t) * \operatorname{HDOP}(t)+\frac{d}{d t}(\text { Failure }(t)) \\
k * U E R E_{Z}(t) * \operatorname{VDOP}(t)+\frac{d}{d t}(\text { Failure }(t))
\end{array}\right]
$$

The detailed GPS error model block diagram is shown in Figure 10. This block diagram represents the GPS error model for one position and velocity channel, total of three identical models with different parameters will be used to generate GPS position and velocity measurements on $x, y$, and $z$ axes. The input signals to the model are the actual position and velocity in the orange color. The purple block represents the model parameter that must be configured by the user. The blue blocks represent the system models. The outputs, measured position and velocity, are represented as green blocks. 


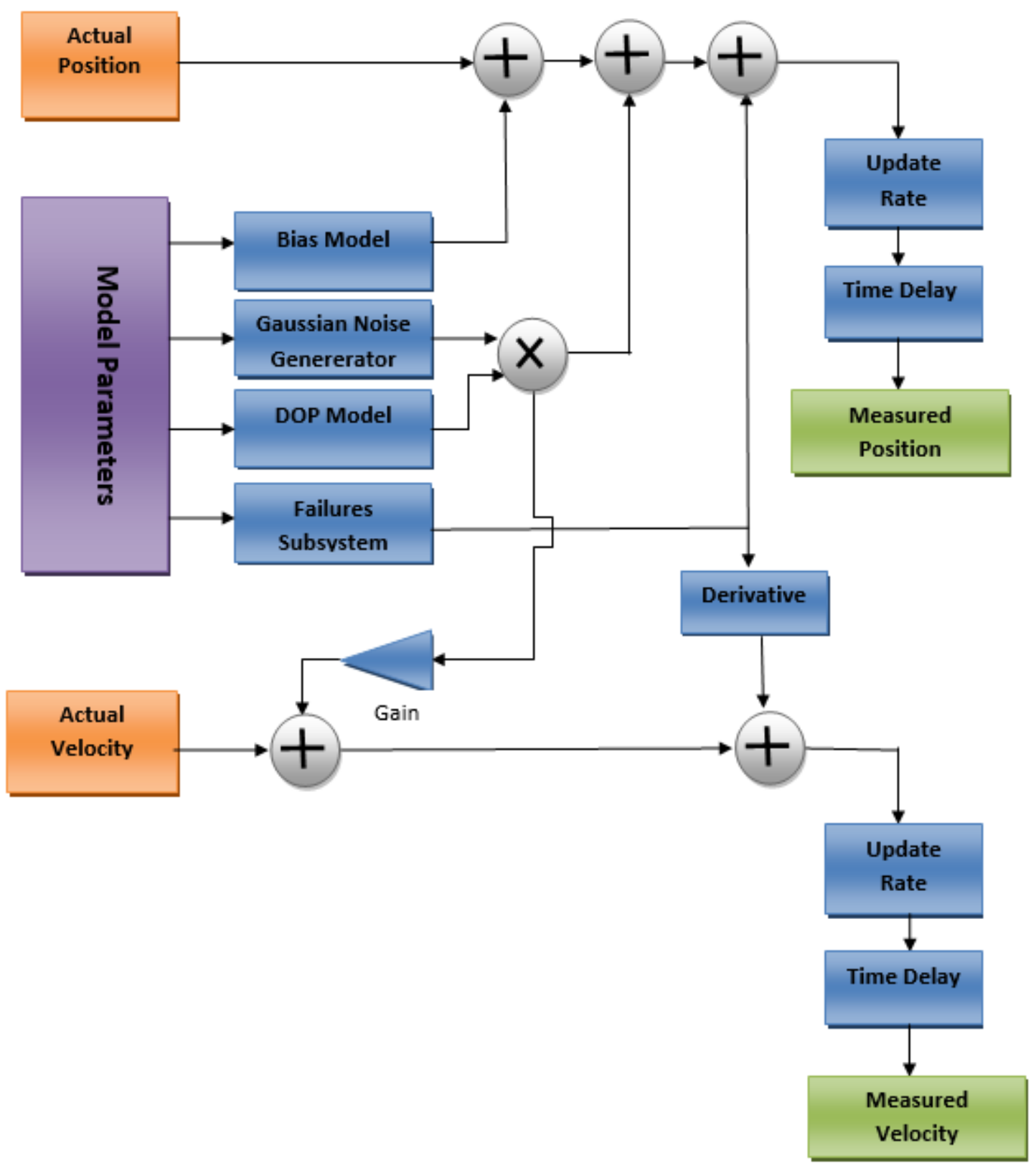

Figure 10: GPS Error Model Detailed Block Diagram 


\subsection{GPS Magnitude Errors}

In this section a detailed description about the GPS error model components is provided along their mathematical expressions.

\subsubsection{The UERE Model}

As seen before, the GPS sources of error will directly affect the receiver - satellite range or the pseudorange measurement. Pseudorange measurement refers to the measured range (R) between one satellite and the receiver [5].

$$
R=r+c * E_{\text {Clk }}+E_{\text {Iono }}+E_{\text {Trop }}+E_{m p}+E_{\text {recv }}
$$

Where, $\mathrm{r}$ is the actual receiver - satellite distance, $\mathrm{c}$ is the speed of light, $E_{C l k}$ is the receiver clock error, $E_{\text {Iono }}$ is the ionosphere error, $E_{\text {Trop }}$ is the troposphere error, $E_{m p}$ is the multipath error, and $E_{r e c v}$ is the receiver measurement error. The measurement errors (e.g. $E_{C l k}, E_{I o n o}$, etc.) will affect each satellite-to-receiver range measurement differently. In addition, the range measurement error for some satellites may exhibit a certain degree of correlation; therefore, a complex method should be used to estimate the range error on the position accuracy [33]. A simplified method to study the effect of the range error assumes that error sources will affect all satellite pseudoranges equally and independently, then the effect of the ranging errors on the positioning error can be studied using the UERE value and a geometric factor $[5,33,36]$. The UERE can be approximated as zero mean Gaussian variable $[5,6]$ and its value can be calculated using the URE and UEE as seen in equation (1). Therefore, the UERE measurement was modeled using Gaussian noise with a mean of zero. To allow for greater flexibility, the variance of each position components was considered to be independent. For example, the UERE value of the $x$ axis position component of STD $x$ will be:

$$
-2 * S T D_{x} \leq U E R E_{x} \leq 2 * S T D_{x}, \text { for } 95 \% \text { of the time }
$$

In the same manner, the $y$-axis UERE value will be:

$$
-2 * S T D_{y} \leq U E R E_{y} \leq 2 * S T D_{y} \text {, for } 95 \% \text { of the time }
$$

and the z-axis position UERE will be 


$$
-2 * S T D_{z} \leq U E R E_{z} \leq 2 * S T D_{z} \text {, for } 95 \% \text { of the time }
$$

\subsubsection{The Dilution of Precision (DOP) Model}

The Dilution of Precision (DOP) is a geometric factor used to state the effect of the UERE on the final GPS receiver position and velocity measurement accuracy. DOP is a dimensionless number that can be calculated using the unit vectors from the receiver to each satellite within view. The smaller the DOP number, the better the geometry, and the better position and velocity precision [33]. The DOP value varies with time due to movement of the receiver or satellites; however, the change of DOP values will be small over time unless the satellite is rising or falling with respect to the receiver [33]. Figure 11 provides an example of 2 hours of measured DOP values for a static GPS receiver.

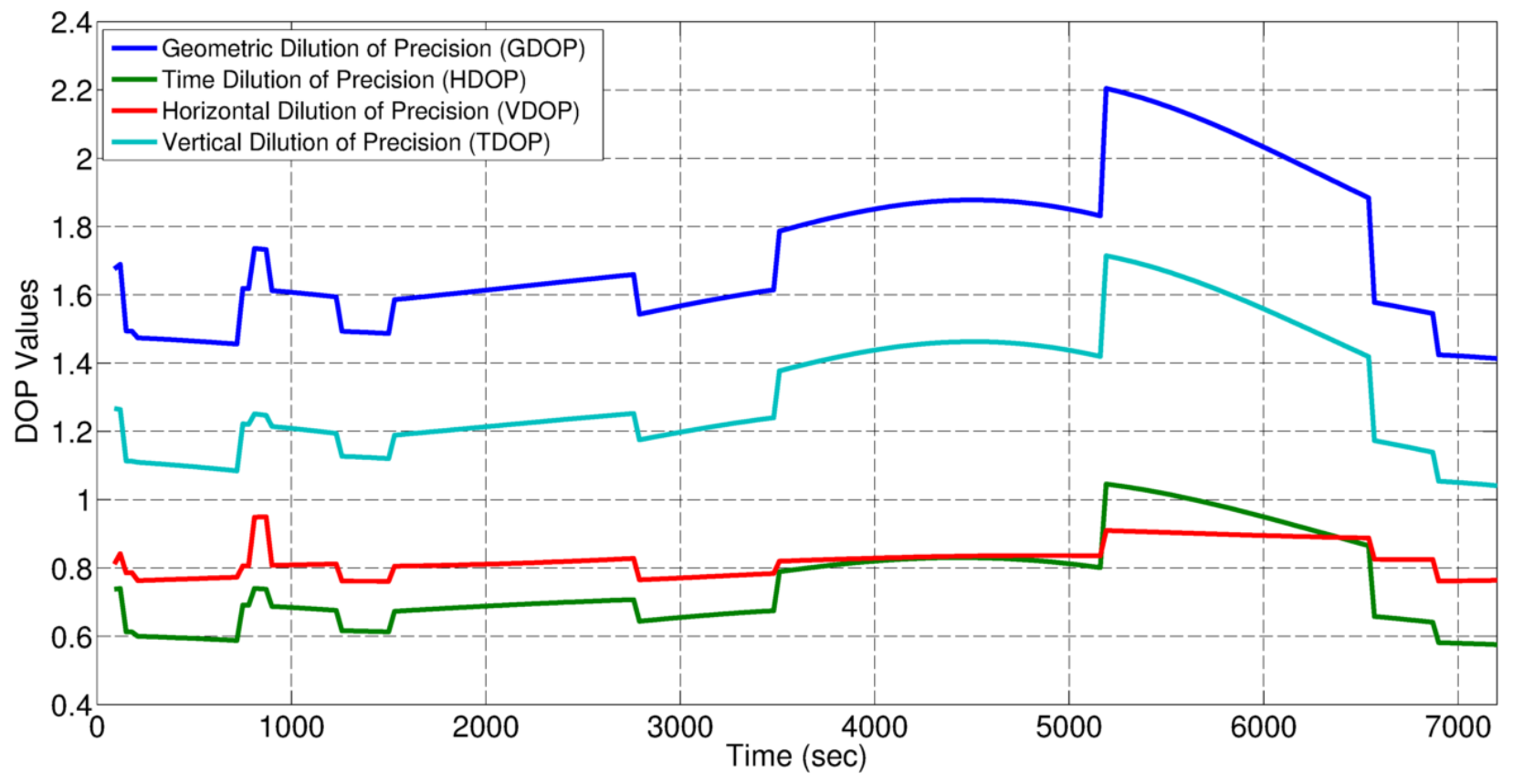

Figure 11: DOP Values for 2 Hours

The HDOP and VDOP were modeled in Simulink using the repeating interpolated sequence block. The repeating interpolated sequence block creates a repeating signal based on the two vectors provided as input. The first $\mathrm{n}$-dimensional row vector provides the values for the repeating sequence, while the second n-dimensional row vector correlates these values to time. For example the following signal shown in Figure 12 is obtained by using a value vector of [1.5 1.75 1.61 .51 .3111 .5 ] and a time vector of [0 200250300150017502250 2500] 


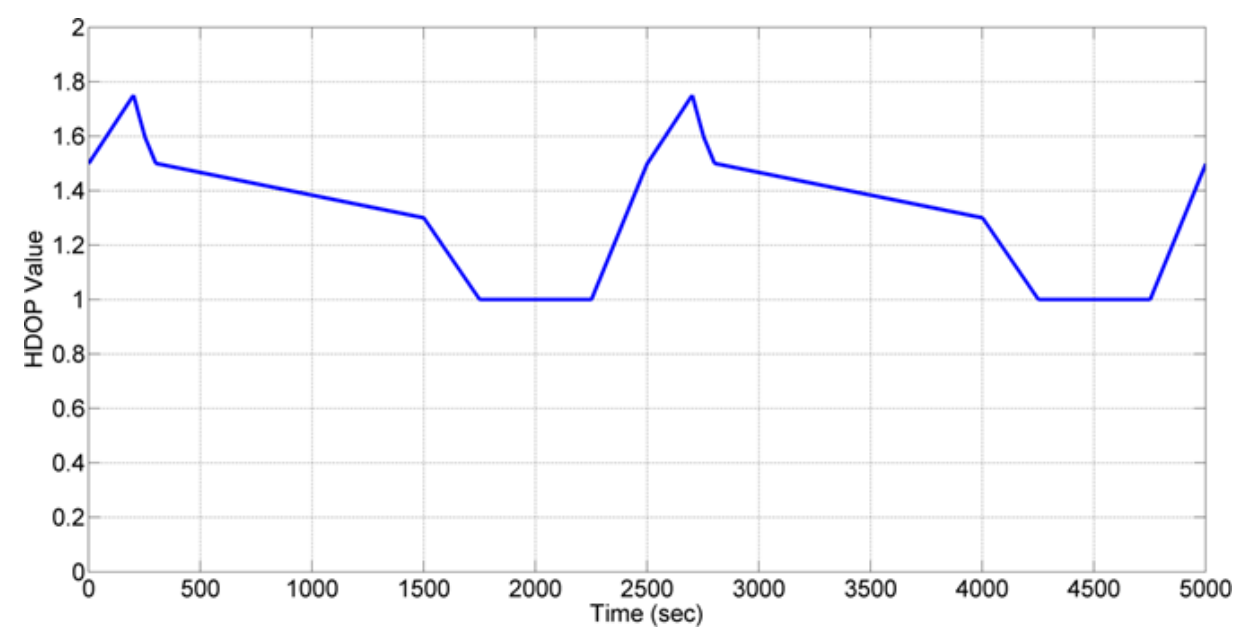

Figure 12: DOP Model Output Example

Let the HDOP values vector be represented as $h=[H(1) H(2) H(3) \ldots H(n)]$, and let corresponding time vector be represented as $t=\left[t_{H}(1) t_{H}(2) t_{H}(3) \ldots t_{H}(n)\right]$, then the output of the HDOP block at time $t$ is given by:

$$
H D O P(t)= \begin{cases}H(1), & t=t_{H}(1) \\ H(1)+\frac{H(2)-H(1)}{t_{H}(2)-t_{H}(1)} *\left(t-t_{H}(1)\right), & t_{H}(1)<t \leq t_{H}(2) \\ H(2)+\frac{H(3)-H(2)}{t_{H}(3)-t_{H}(2)} *\left(t-t_{H}(2)\right), & t_{H}(1)<t \leq t_{H}(2) \\ \cdot & \cdot \\ \cdot & \cdot \\ H(n-1)+\frac{H(n)-H(n-1)}{t_{H}(n)-t_{H}(n-1)} *\left(t-t_{H}(n-1)\right), & t_{H}(n-1)<t \leq t_{H}(n)\end{cases}
$$

where $\operatorname{HDOP}\left(t+n * t_{H}(n)\right)=\operatorname{HDOP}(t), \mathrm{n}=[1,2,3, \ldots]$;

Let the VDOP values vector be represented as $v=[V(1) V(2) V(3) \ldots V(n)]$, and let corresponding time vector be represented as $t=\left[t_{V}(1) t_{V}(2) t_{V}(3) \ldots t_{V}(n)\right]$, then the output of the VDOP block at time $t$ is given by:

$$
V D O P(t)= \begin{cases}V(1), & t=t_{V}(1) \\ V(1)+\frac{V(2)-V(1)}{t_{V}(2)-t_{V}(1)} *\left(t-t_{V}(1)\right), & t_{V}(1)<t \leq t_{V}(2) \\ V(2)+\frac{V(3)-V(2)}{t_{V}(3)-t_{V}(2)} *\left(t-t_{V}(2)\right), & t_{V}(1)<t \leq t_{V}(2) \\ \cdot & \cdot \\ \cdot & \cdot \\ V(n-1)+\frac{V(n)-V(n-1)}{t_{V}(n)-t_{V}(n-1)} *\left(t-t_{V}(n-1)\right), & t_{V}(n-1)<t \leq t_{V}(n)\end{cases}
$$


where $\operatorname{VDOP}\left(t+n * t_{H}(n)\right)=\operatorname{VDOP}(t), \mathrm{n}=[1,2,3, \ldots]$;

\subsubsection{GPS Position Bias Model}

The value of the error mean varies for different longitude, latitude, and altitude combinations. The value of the bias is generally small on the latitude direction, and larger on the altitude direction. An example of real GPS position error is shown in Figure 13 below. This GPS data was downloaded from the IGS website and it was processed using the gLAB software $[28,30]$.

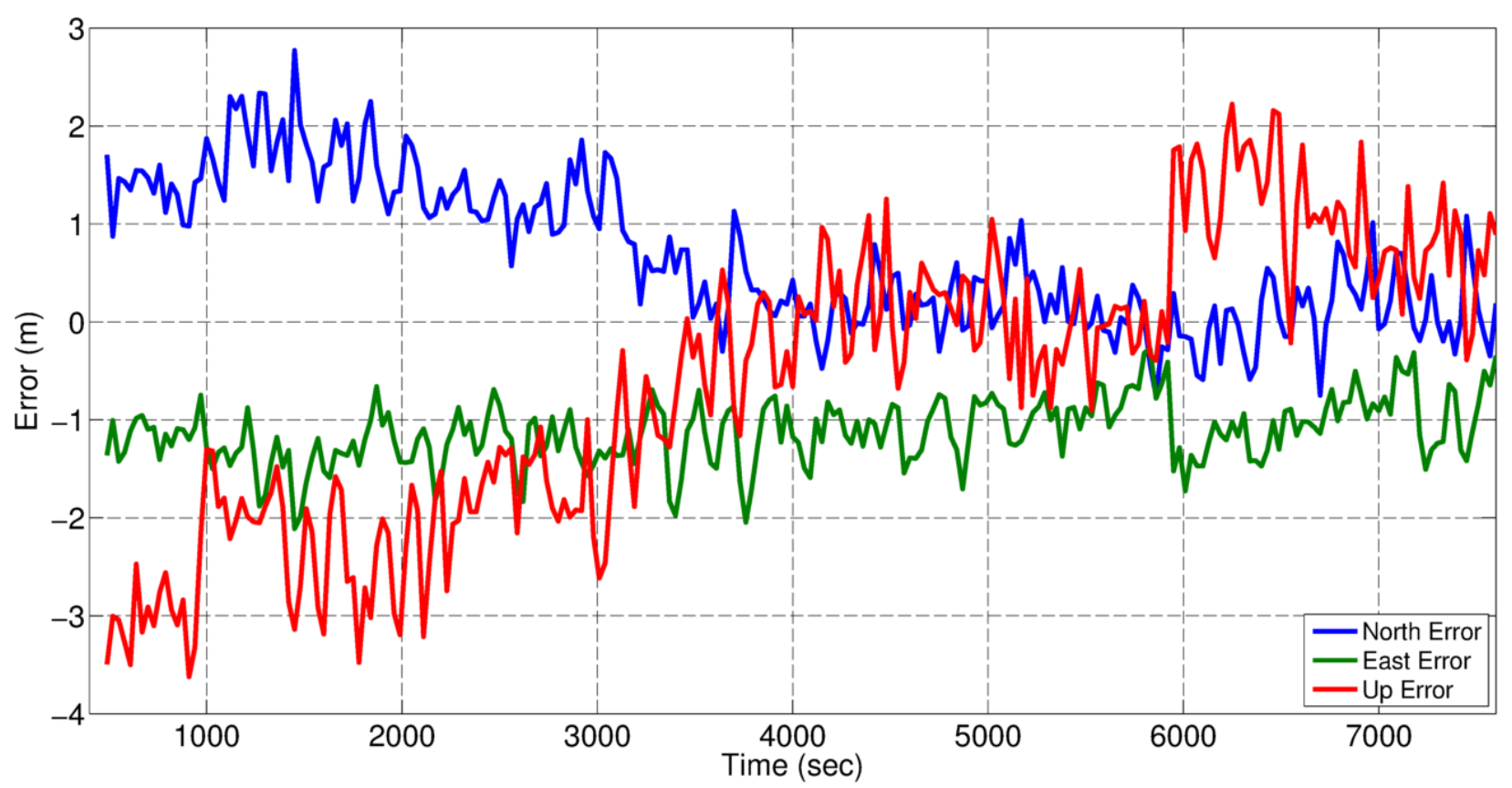

Figure 13: Actual GPS Position Error [28, 30]

Based upon the GPS data analysis which was conducted, the mean value of the GPS position error is not zero. The analysis of GPS positioning involved using GPS SPS data from six different distinct days. The analysis involved measuring maximum horizontal error, maximum vertical error, the standard deviation of horizontal error, the standard deviation of vertical error, the mean of horizontal error, and mean of the vertical error. The summary of this analysis is presented in Figure 14 below. 

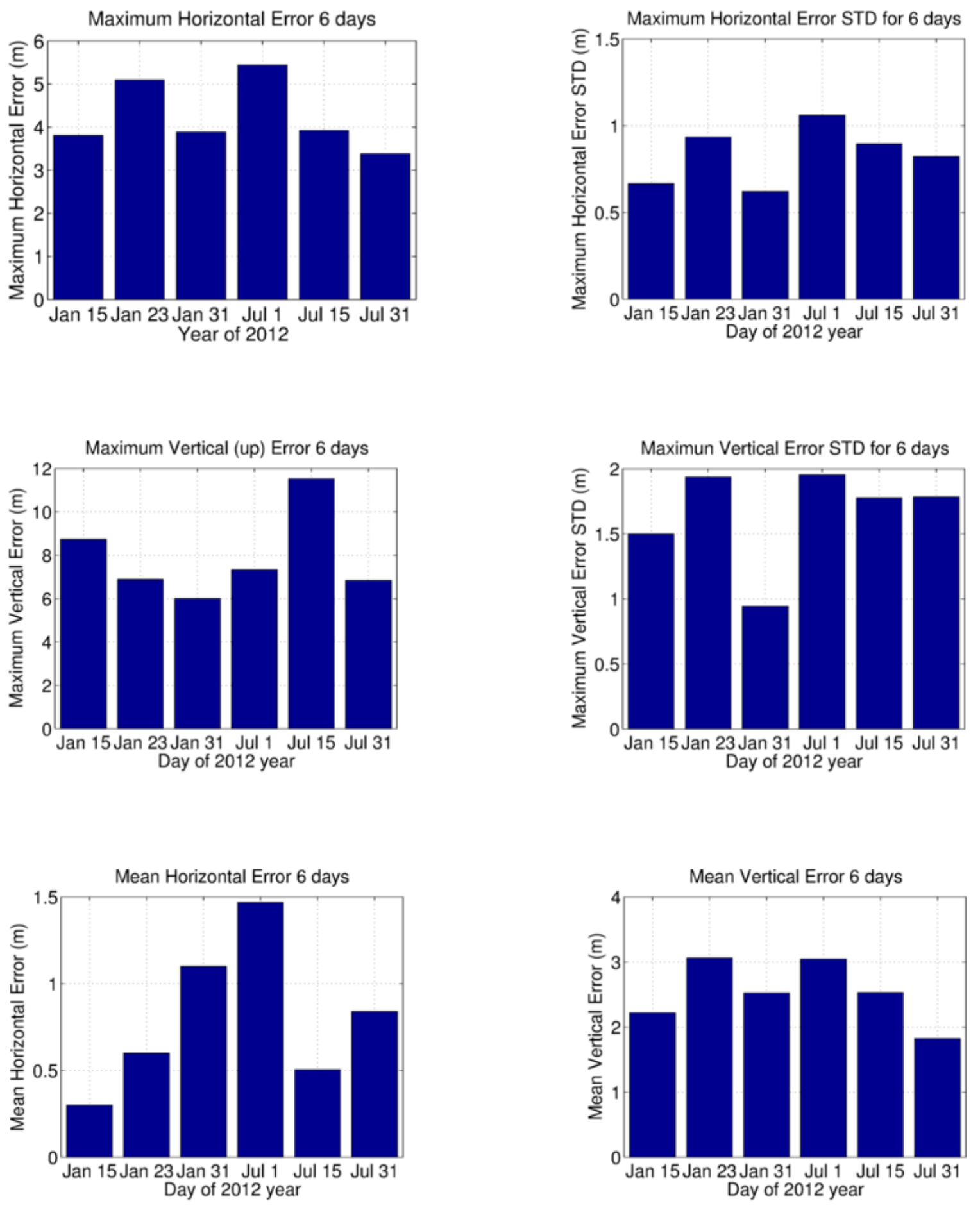

Figure 14: GPS Error Analysis Summary

The GPS position error bias was also modeled in Simulink using the repeating interpolated sequence block. Each of the position error components was modeled separately. Let the $\alpha$-axis bias value vector be represented as $B_{\alpha}=\left[B_{\alpha}(1) B_{\alpha}(2) B_{\alpha}(3) \ldots B_{\alpha}(n)\right]$, where $\alpha$ can be $\mathrm{x}, \mathrm{y}$, or 
$z$ and let the corresponding time vector be represented as $t_{\alpha B}=$ $\left[t_{\alpha B}(1) t_{\alpha B}(2) t_{\alpha B}(3) \ldots t_{\alpha B}(n)\right]$, then the bias of $\alpha$-axis error at time $=t$ will be:

$$
\operatorname{Bias}_{\alpha}(t)= \begin{cases}B_{\alpha}(1), & t=t_{\alpha B}(1) \\ B_{\alpha}(1)+\frac{B_{\alpha}(2)-B_{\alpha}(1)}{t_{\alpha B}(2)-t_{\alpha B}(1)} *\left(t-t_{\alpha B}(1)\right), & t_{\alpha B}(2) \geq t>t_{\alpha B}(1) \\ B_{\alpha}(2)+\frac{B_{\alpha}(3)-B_{\alpha}(2)}{t_{\alpha B}(3)-t_{\alpha B}(2)} *\left(t-t_{\alpha B}(2)\right), & t_{\alpha B}(3) \geq t>t_{\alpha B}(2) \\ \cdot & \cdot \\ \cdot & \cdot \\ \cdot & \cdot \\ B_{\alpha}(n-1)+\frac{B_{\alpha}(n)-B_{\alpha}(n-1)}{t_{\alpha B}(n)-t_{\alpha B}(n-1)}\left(t-t_{\alpha B}(n-1)\right), & t_{\alpha B}(n) \geq t>t_{\alpha B}(n-1) \\ B_{\alpha}(n), & t=t_{\alpha B}(n)\end{cases}
$$

where $\operatorname{Bias}_{\alpha}\left(t+n * t_{\alpha B}\right)=\operatorname{Bias}_{\alpha}(t)$, where $\mathrm{n}=[1,2,3, \ldots]$.

\subsubsection{GPS Position Failures Model}

According to the GPS performance standards document and according to table (7), the GPS UERE might suffer four types of failures: excessive noise, step, ramp, or sinusoidal output variation. The failure subsystem in the model is responsible of generating the required failures according to the provided failure parameters: failure type (type), failure value $\left(F_{V}\right)$, start time $\left(t_{S}\right)$, duration (dur), and the frequency (Freq) in the case of sinusoidal failures. Note that the failure value will represent the standard deviation (if excessive noise is simulated), the magnitude of the step (for the step failure), the slope of the linear variation (for the ramp failure), or the amplitude (for the sinusoidal failure). In other words, the failure model output FM at time $t$ will be:

$$
F M(t)= \begin{cases}0, & \text { type }=\text { "None" } \\ -2 * F_{V} \leq F_{M} \leq 2 * F_{V}, & \text { type }=\text { "Noise" and } t_{S} \leq t \leq t_{S}+\text { dur } \\ F_{V}, & \text { type }=" \text { Step" and } t_{S} \leq t \leq t_{S}+\text { dur } \\ F_{V}\left(t-t_{S}\right), & \text { type }=" \text { Ramp" and } t_{S} \leq t \leq t_{S}+d u r \\ F_{V} * \sin (\text { freq } * t), & \text { type }=\text { "Sinusoidal" and } t_{S} \leq t \leq t_{S}+d u r\end{cases}
$$

\subsubsection{Update Interval and Time Delay}

The update Interval is the amount of time the GPS receiver takes to process and send new position or velocity readings. Different GPS receivers have different update Interval; the values of update Intervals vary significantly from 0.02 to $1 \mathrm{sec}$ or even larger. Some receivers may have a position update Interval smaller than the velocity update rate. Let the receiver update Interval 
be represented as $\delta t$ and the input signal be defined as Input, then the output signal Output at time equal $t$ will be:

$$
\operatorname{Output}(t)=I(n * \delta t) \quad \text { if } n * \delta t \leq t<n * \delta t \quad \text { where } \mathrm{n}=[1,2,3, \ldots . .] .
$$

Time Delay is the time difference between receiver signal acquisition and the time the measurement was made. The time delay does not affect stationary or low-speed moving objects; however, the time delay will affect high speed moving vehicles (e.g. aircraft). The time delay will add to position error by giving old position measurements, and this error will become larger in the case of high-speed vehicles. Assume the receiver time delay be represented as $\tau$, the input signal be defined as Input, and the output signal as Output The regular delay function will assume Output to be zero for any time $t$ less than $\tau$, but the aircraft position cannot be zero at any time; thus, a special delay function was used where

$$
\operatorname{Output}(t)=\left\{\begin{array}{cc}
\operatorname{Input}(t) & t \leq \tau \\
\operatorname{Input}(t-\tau) & t>\tau
\end{array}\right.
$$

\subsection{Simulink Implementation of the GPS Error Model}

The Simulink implementation of the GPS error model is shown in Figure 15 below. Three Gaussian noise generators were used to generate the required UERE. The output of the Gaussian generator will be multiplied by HDOP for horizontal position accuracy, and by the VDOP for the vertical position accuracy. The product of the Gaussian noise generator will be scaled for corresponding velocity accuracy by a simple gain block in the velocity subsystem block. Biases and failures will be applied to each position channel, while the failure time derivative will be applied on the velocity channel. 


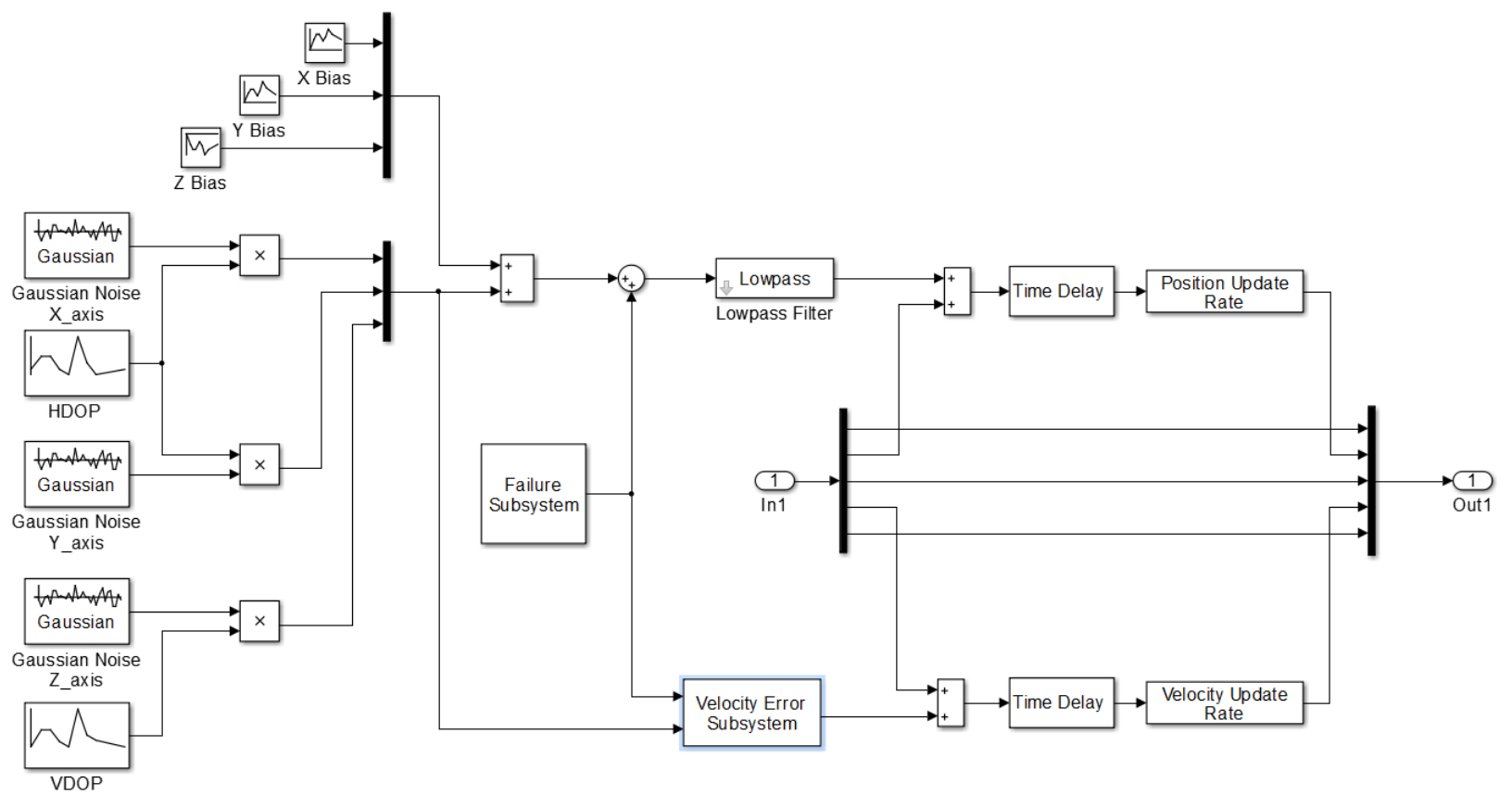

Figure 15: GPS Error Model Simulink Implementation

Simple low pass filters were used on both position and velocity error channels. The update rate transaction and the time delay subsystems applied on the measured position and velocity. Both position and velocity channels will have delayed outputs by the same amount; however, they might have different update Intervals.

A Graphical User Interface (GUI) was used to initialize all required parameters needed to generate the proper position and velocity error. These parameters include error standard deviation, error biases, the HDOP and VDOP vectors, the velocity to position standard deviation ratio, position and velocity update Intervals, time delay, and failure type, if any. The GUI also simplifies the process of entering the parameter through choosing pre-set parameters using the pop up menu, or by using check boxes that minimize the number of needed parameters; furthermore, Simulink subsystem mask was designed for the GPS error model for easier parameter access during the simulation. Figure 16 show the GPS error model GUI, while Figure 17 and Figure 18 display different screen shots for the model mask. 


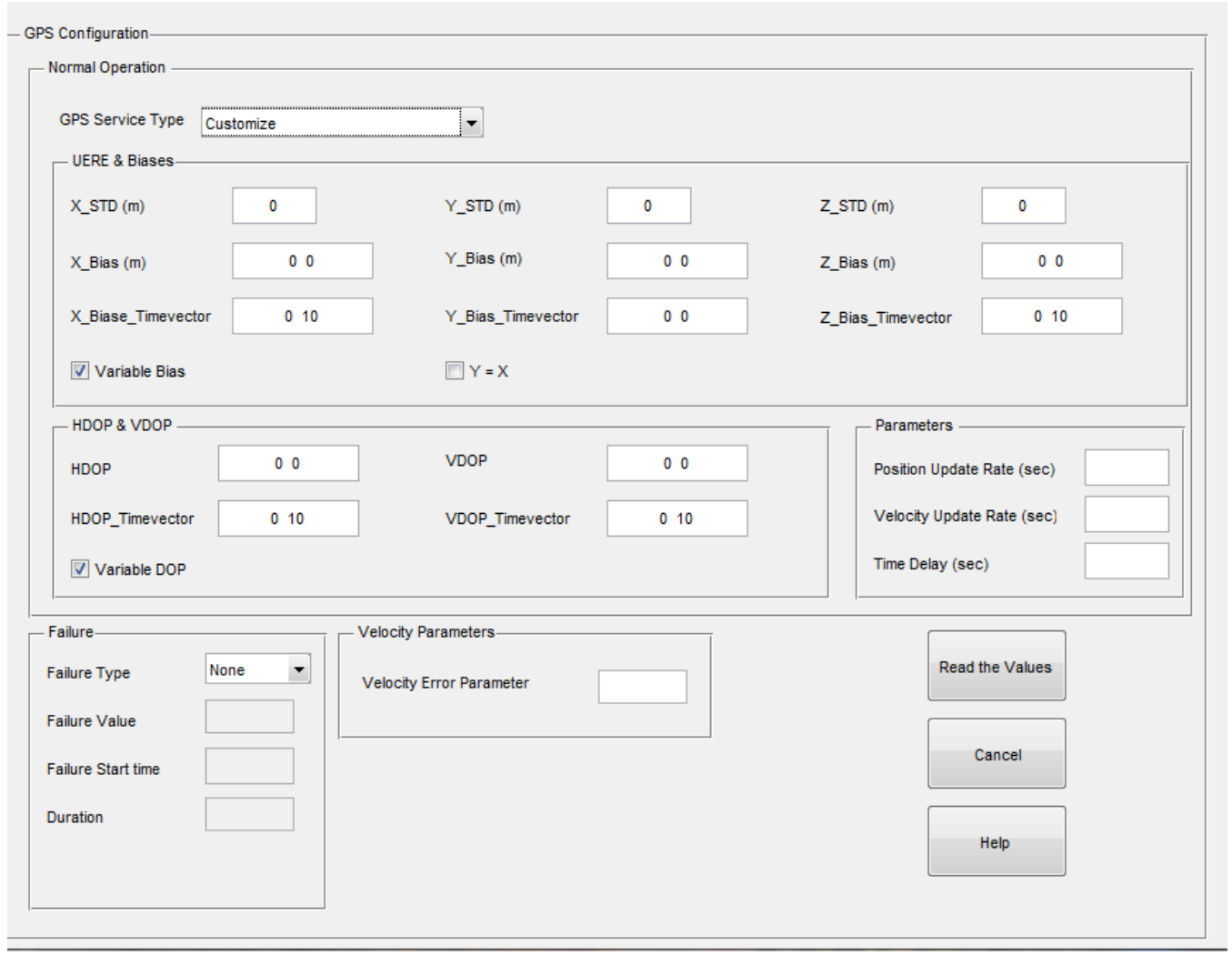

Figure 16: GPS Error Model GUI

\begin{tabular}{|c|c|c|c|c|c|}
\hline \multicolumn{6}{|c|}{ Function Block Parameters: GPS Block1 } \\
\hline \multicolumn{6}{|l|}{ GPS Block (mask) } \\
\hline \multicolumn{6}{|c|}{$\begin{array}{l}\text { GPS Block adds a random gaussian and ( constant or variable bias ) } \\
\text { error to the three position axes. } \\
\text { you can change the variance of the noise by the DOP factor, HDOP will } \\
\text { affect both } x \text { and } y \text { axes, while VDOP will affect only z axis. } \\
\text { Note: Press APPLY so the code can verify the inputs }\end{array}$} \\
\hline System Type & $\mathrm{X}$ axis & $Y$ axis & $Z$ axis & DOP & Failure \\
\hline Operation Mode & Custom & & & & $\boldsymbol{\nabla}$ \\
\hline \multicolumn{6}{|c|}{ Position Update Rate (sec) } \\
\hline \multicolumn{6}{|l|}{0.02} \\
\hline \multicolumn{6}{|c|}{ Velcoity Update Rate (sec) } \\
\hline \multicolumn{6}{|l|}{0.02} \\
\hline \multicolumn{6}{|l|}{ Time Delay (sec) } \\
\hline \multicolumn{6}{|l|}{0.04} \\
\hline \multicolumn{6}{|c|}{ Velocity to Position Standard Deviation ratio } \\
\hline \multicolumn{6}{|l|}{0.3} \\
\hline & OK & & ncel & Help & Apply \\
\hline
\end{tabular}

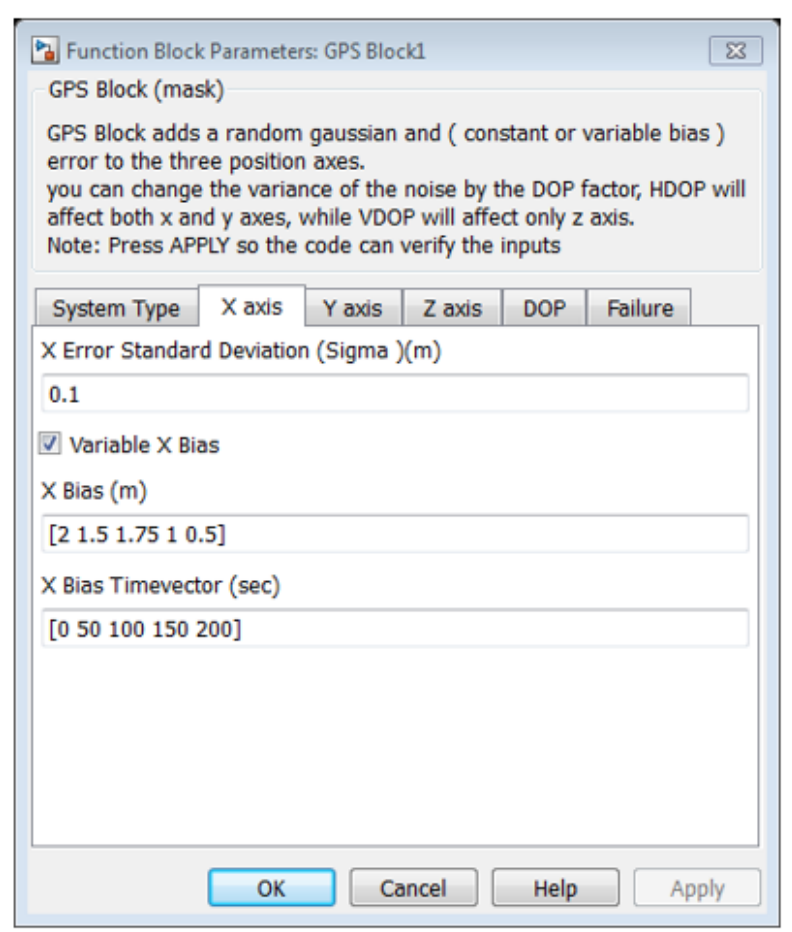

Figure 17: GPS Error Model Mask (Picture No.1) 

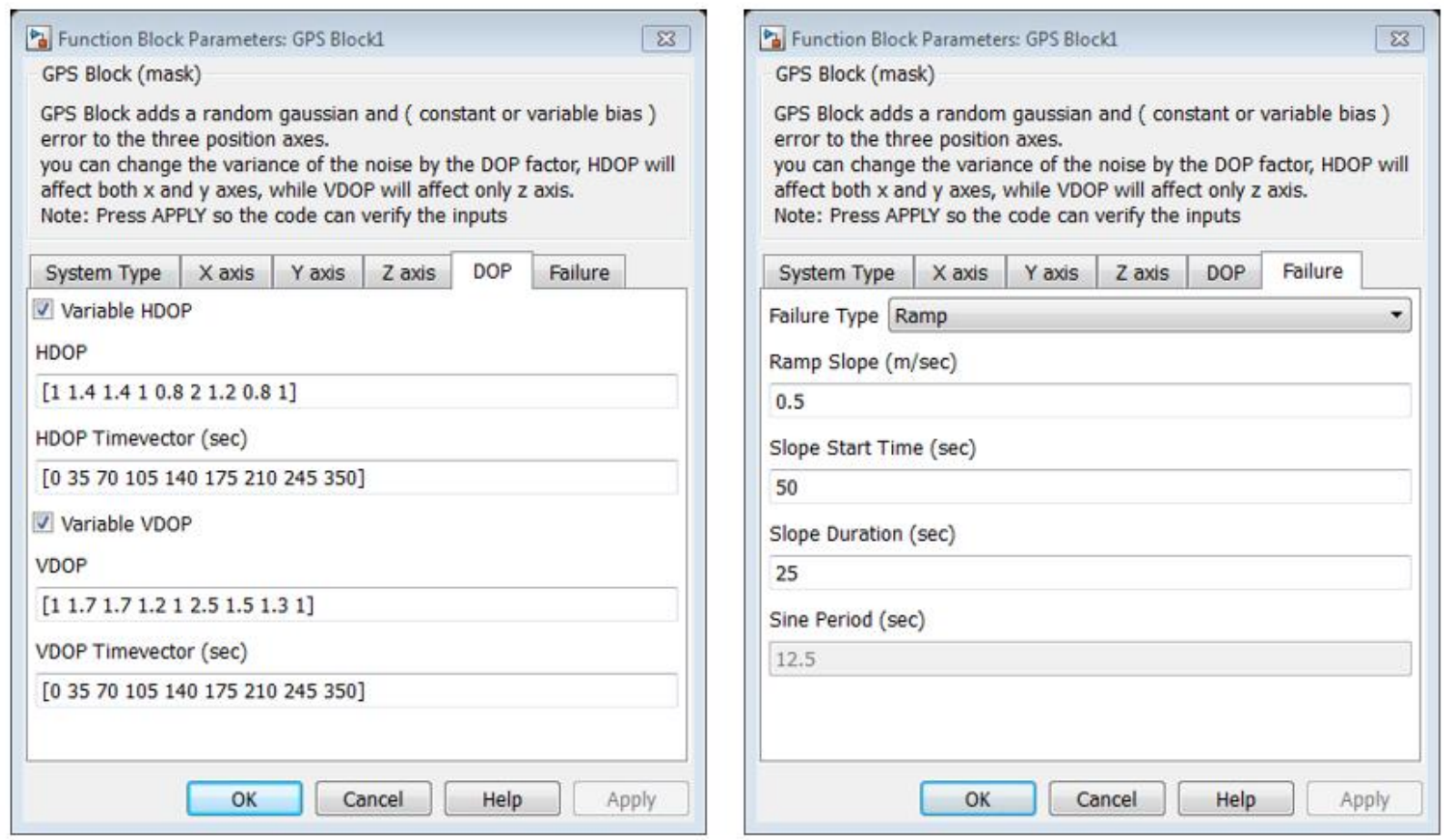

Figure 18: GPS Error Model Mask (Picture No.2)

\subsection{Model Verification}

A) Simulation inspection: in this verification approach, the model was used within the simulation environment to generate different position and velocity errors, then it was verified by inspection that the model is generating the expected outputs. Different failure operation was considered too, and both position and velocity errors were verified to have the intended characteristics. Figure 19 and Figure 20 illustrate an example of this approach.

In this example a ramp failure to the position is simulated at time equals 50 seconds for a duration of 25 seconds. As can be seen in the above figures, it can be easily verified that the position error is computed as expected; furthermore, the above figure also can verify the position-velocity error relationship. 


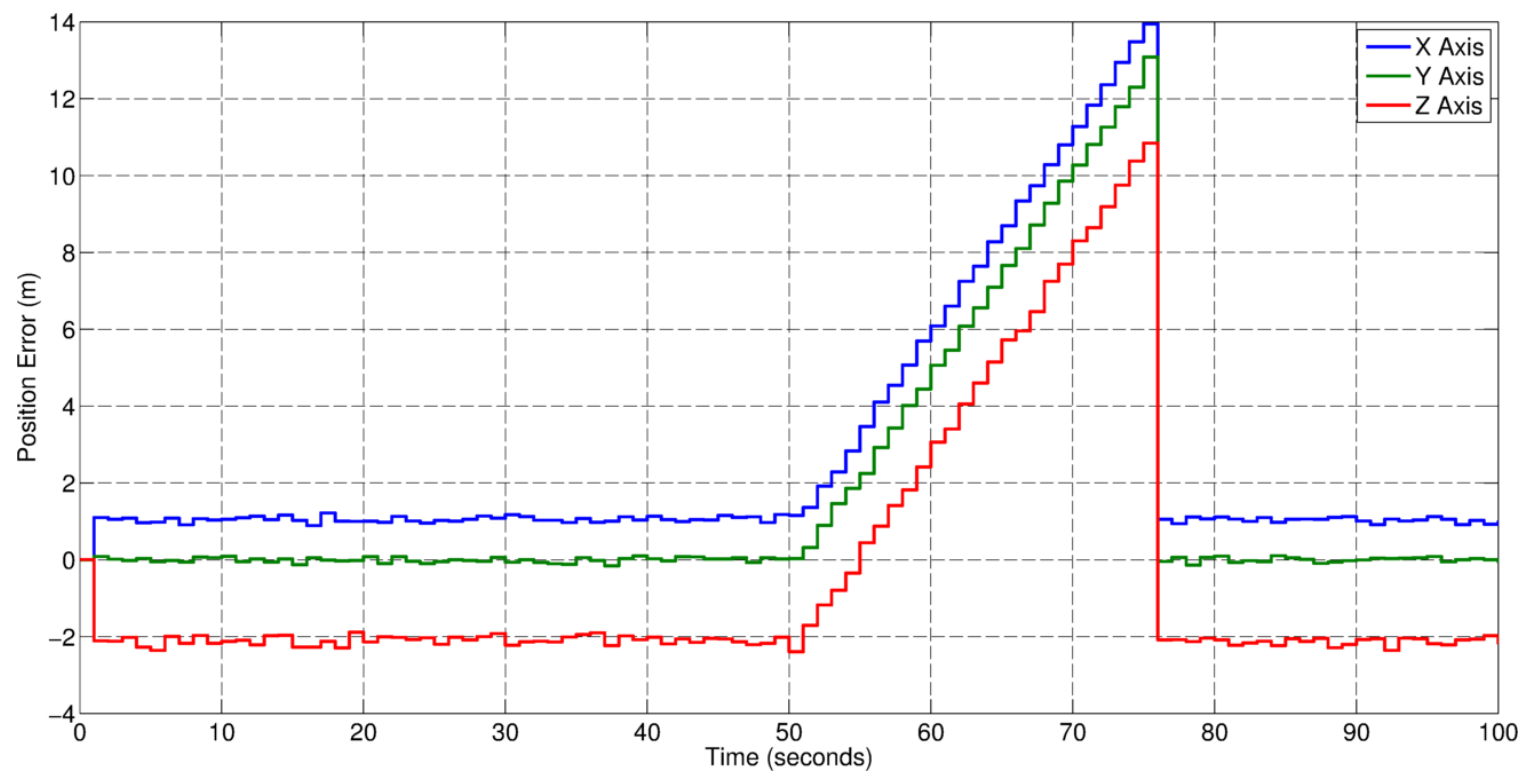

Figure 19: GPS Error Model Position Error Example

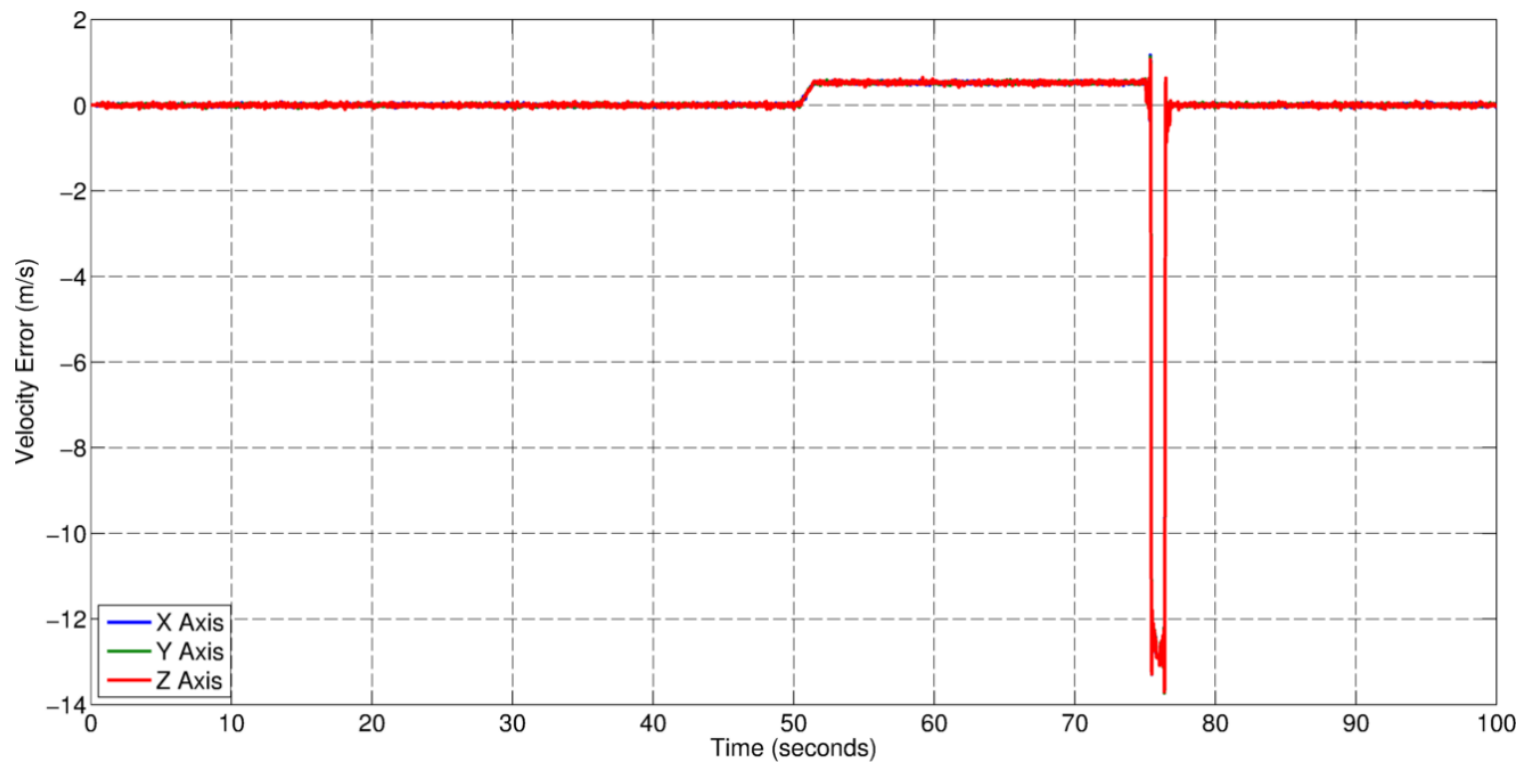

Figure 20: GPS Error Model Velocity Error

B) The actual GPS error verification approach: this model verification process involved reading the actual GPS position error data using the gLAB software. The error data were transformed into GPS error model parameters by Matlab, then finally a comparison between the original error statistics and the GPS block generated error statistics was performed. Figure 21 shows an example of a real GPS position error and Figure 22 displays the GPS block generated error. 


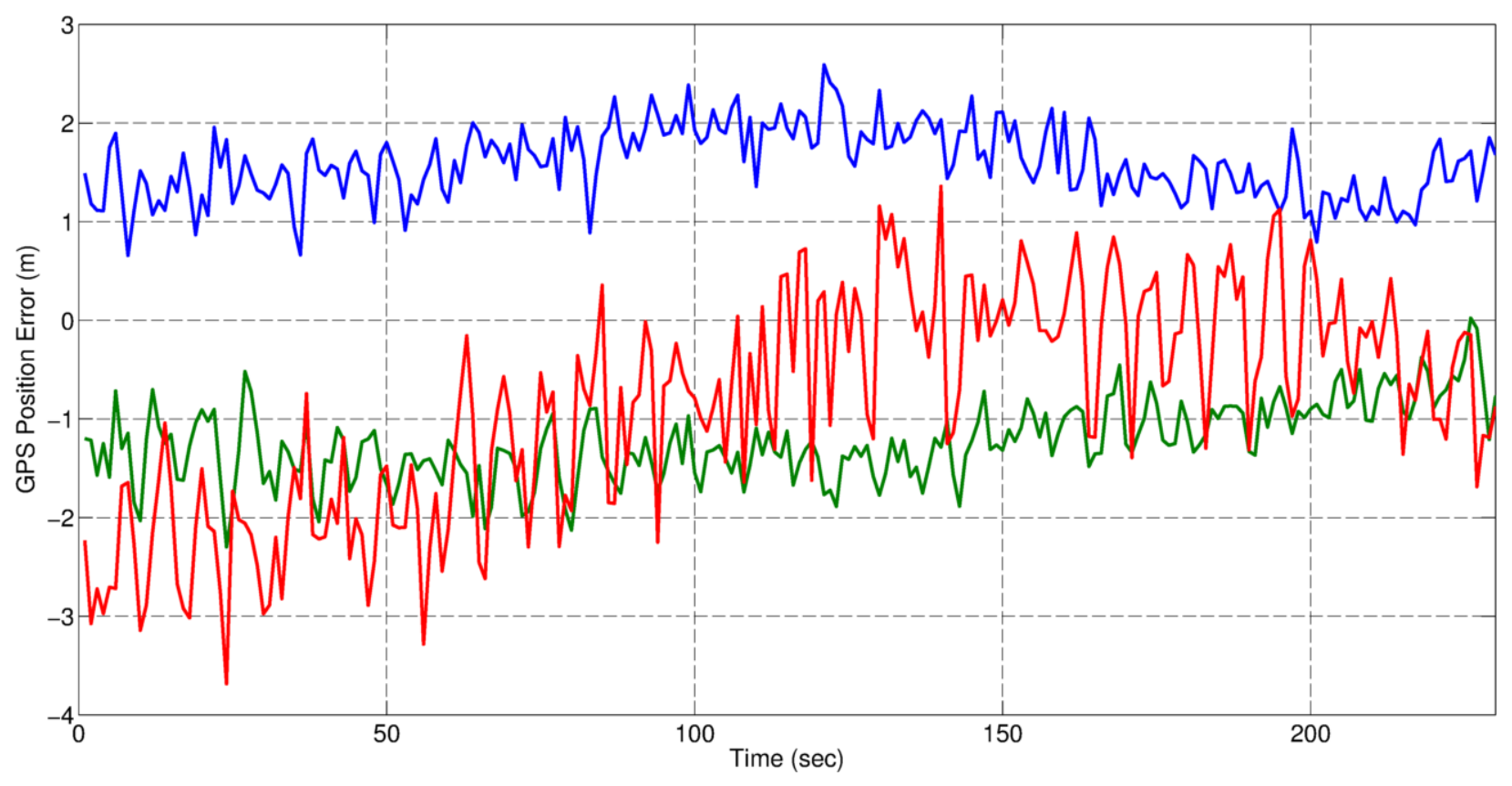

Figure 21: GPS Real Position Error [28, 30]

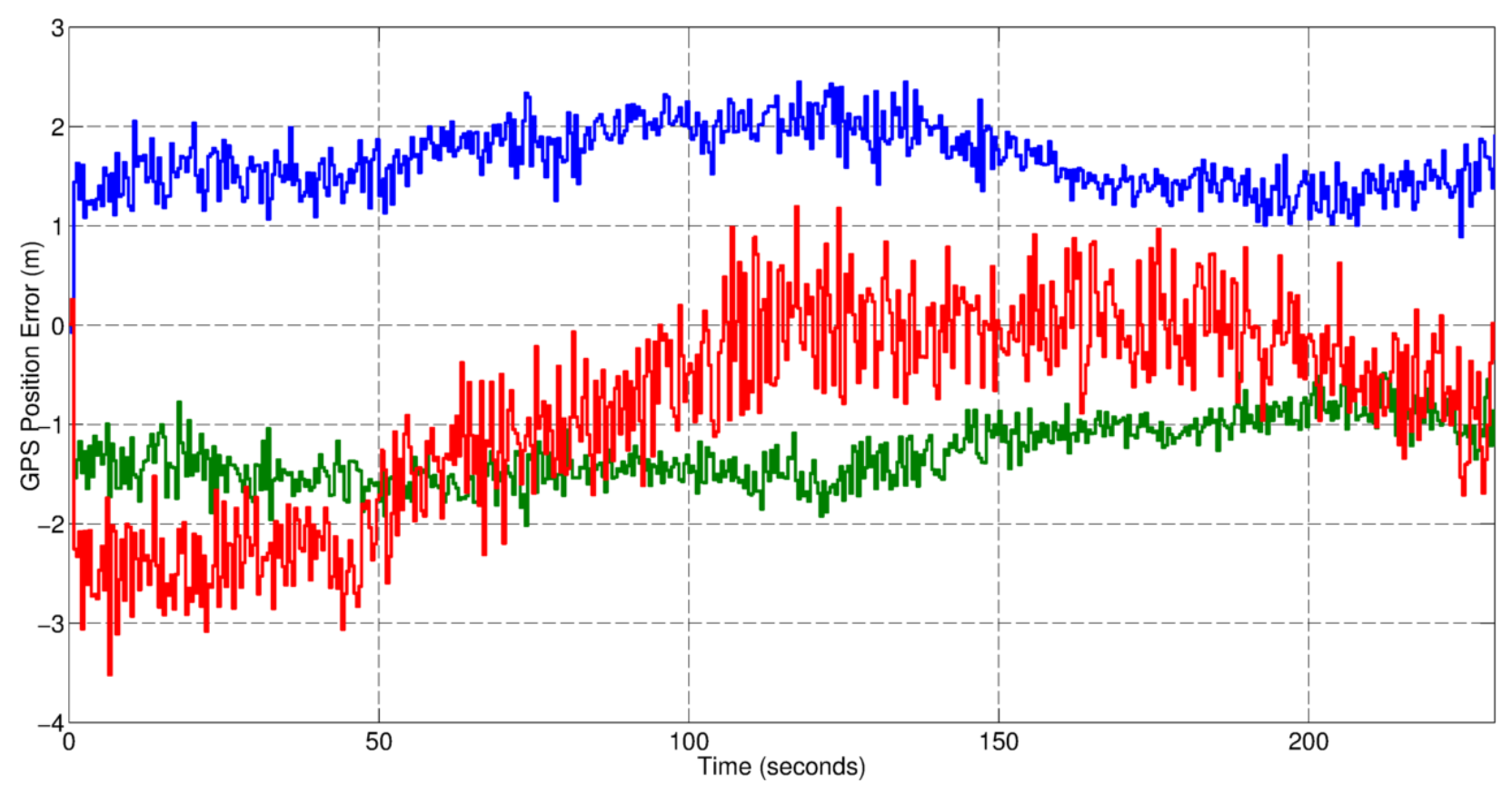

Figure 22: GPS Error Model Generated Position Error

Table 8 display a statistical comparison between the actual GPS position error and the regenerated error. 


\begin{tabular}{|c|c|c|}
\hline & Real GPS Error & Re-Generated Error \\
\hline Standard Deviation & {$[0.350 .391 .09]$} & {$[0.330 .311 .01]$} \\
\hline Mean & {$[1.5-1.20-0.86]$} & {$[1.65-1.28-0.82]$} \\
\hline Maximum & {$[2.590 .021 .35]$} & {$[2.440 .111 .19]$} \\
\hline
\end{tabular}

Table 8: Statistical Comparison between Actual and Regenerated GPS Position Error

The model verification process proves that the model has sufficient parameter and good structure capable of producing errors very similar to real GPS position error; furthermore, the GPS block parameter can be set using the verification process to analyze the controllers' performance against real GPS errors. 


\section{WVU UAV Simulation Environment}

The WVU UAV Simulation Environment has been implemented in MATLAB Simulink [41] (Figure 23), which facilitates the design, implementation, and comparison of autonomous flight control laws.

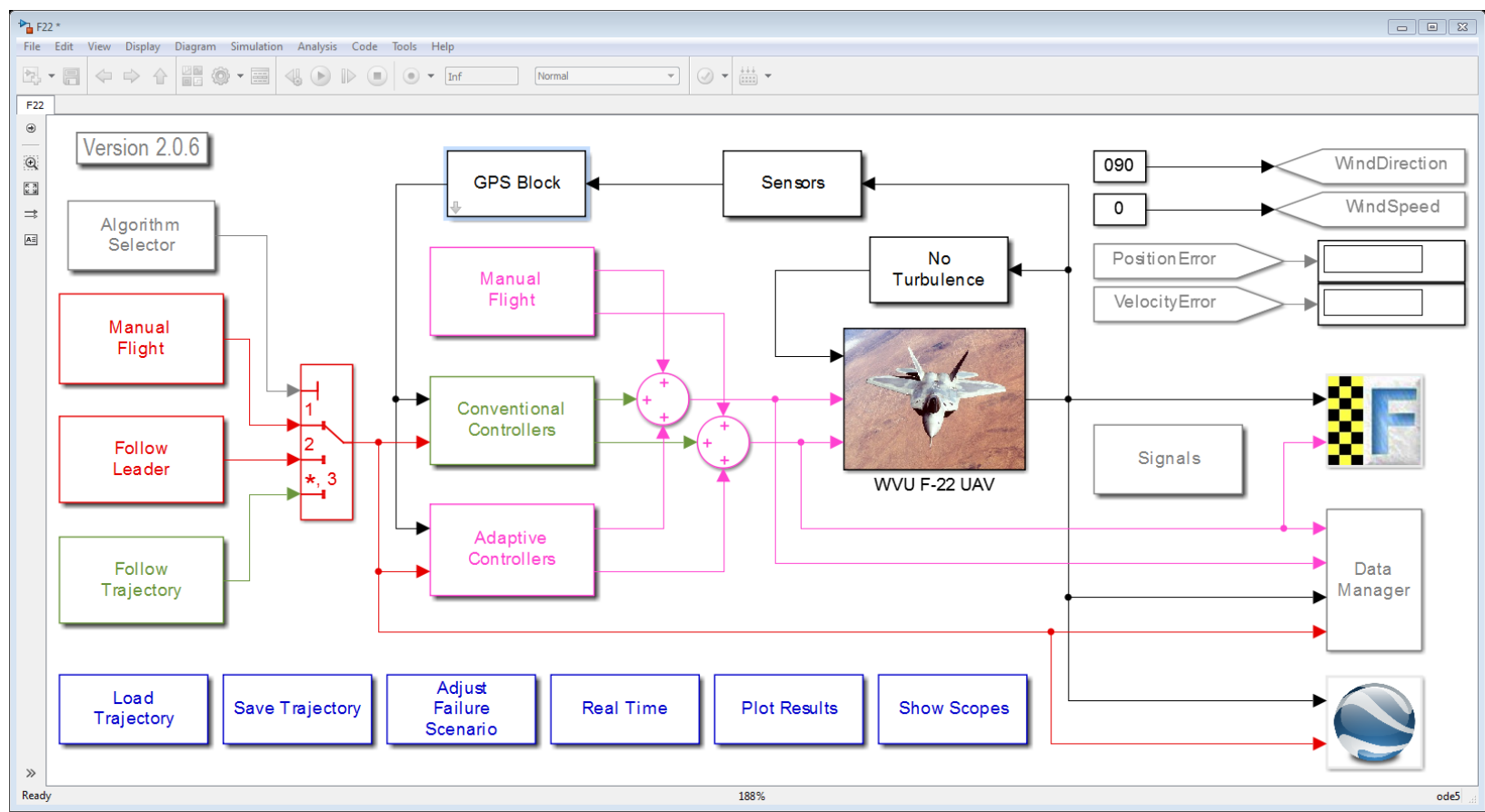

Figure 23: WVU Simulation Environment Screenshot

The simulation environment utilizes FlightGear (Figure 24), an open source aircraft visualization software, for visualization. Furthermore the simulation environment was interfaced with a custom map generation and visual feedback program, called UAVDashboard (Figure 25). 


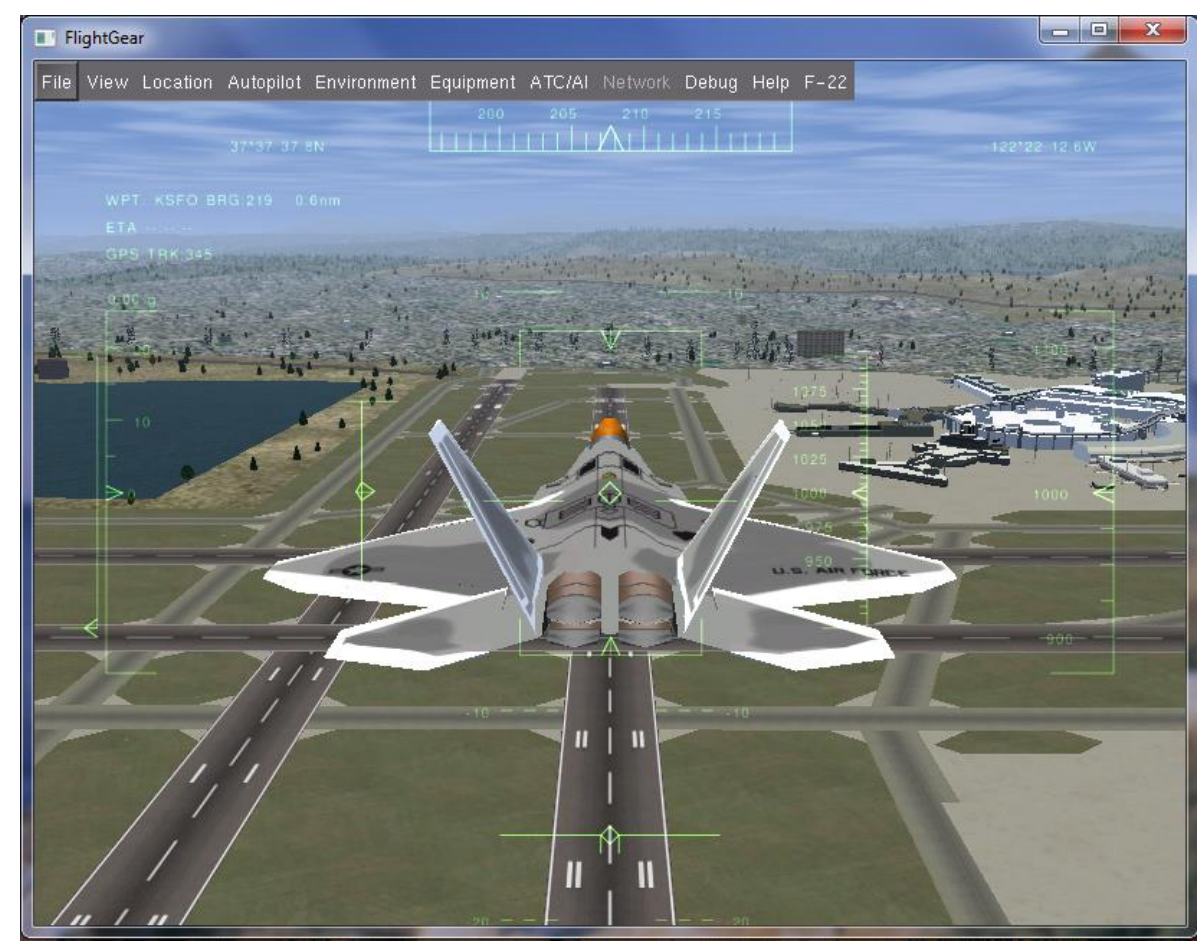

Figure 24: FLIGHTGEAR Screenshot

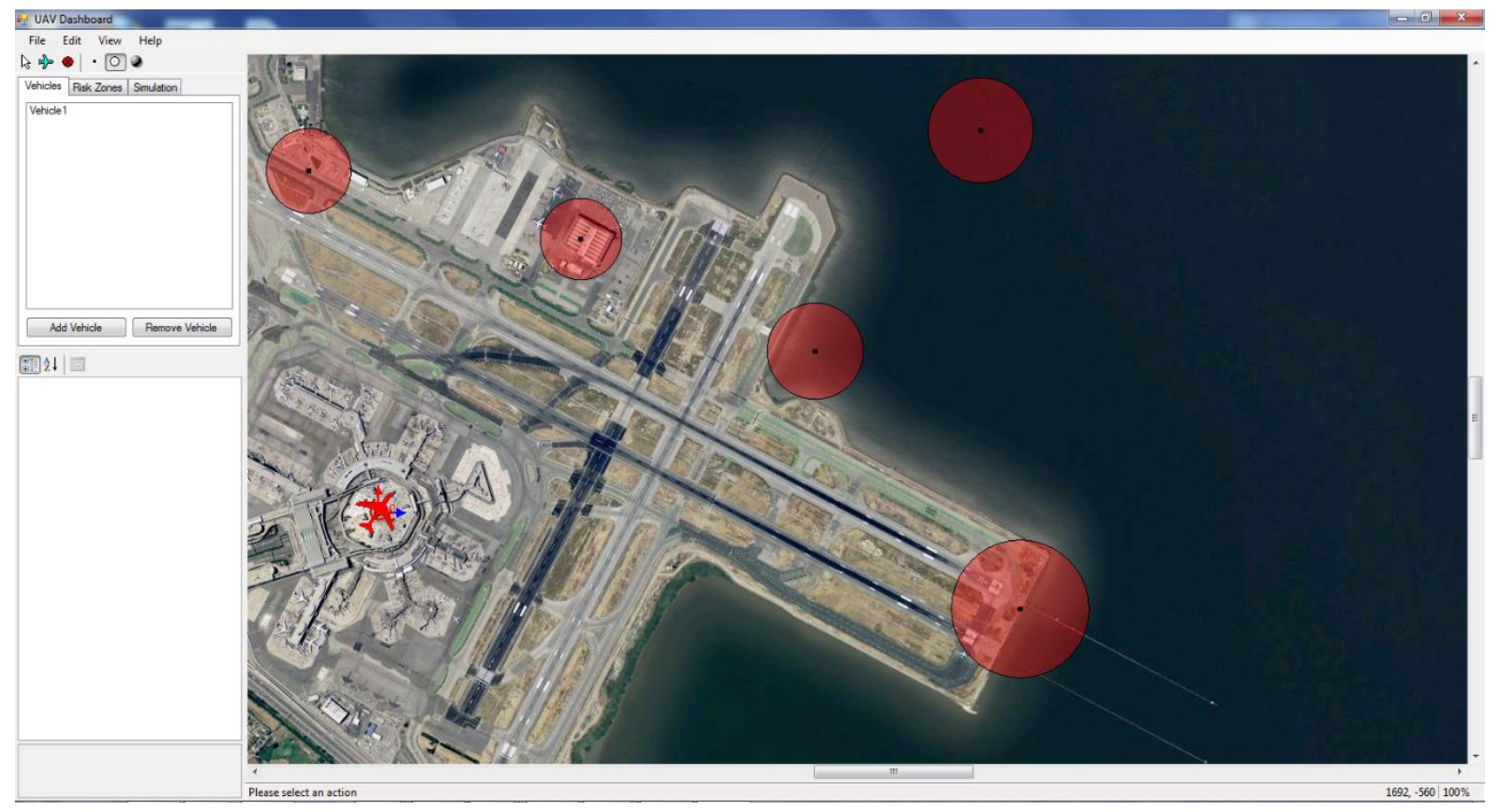

Figure 25: UAVDashboard screenshot

\subsection{The Graphical User Interface (GUI)}

To initialize all the parameter required to run the simulation, a graphical user interface has been implemented (Figure 26). From the GUI, the user has the option to select the aircraft model, the trajectory planning algorithm, the controller, and a set of parameter for normal and abnormal 
operations. Once all the desired parameters have been selected, the FlightGear and UAVDashboard software can be lunched from the GUI.

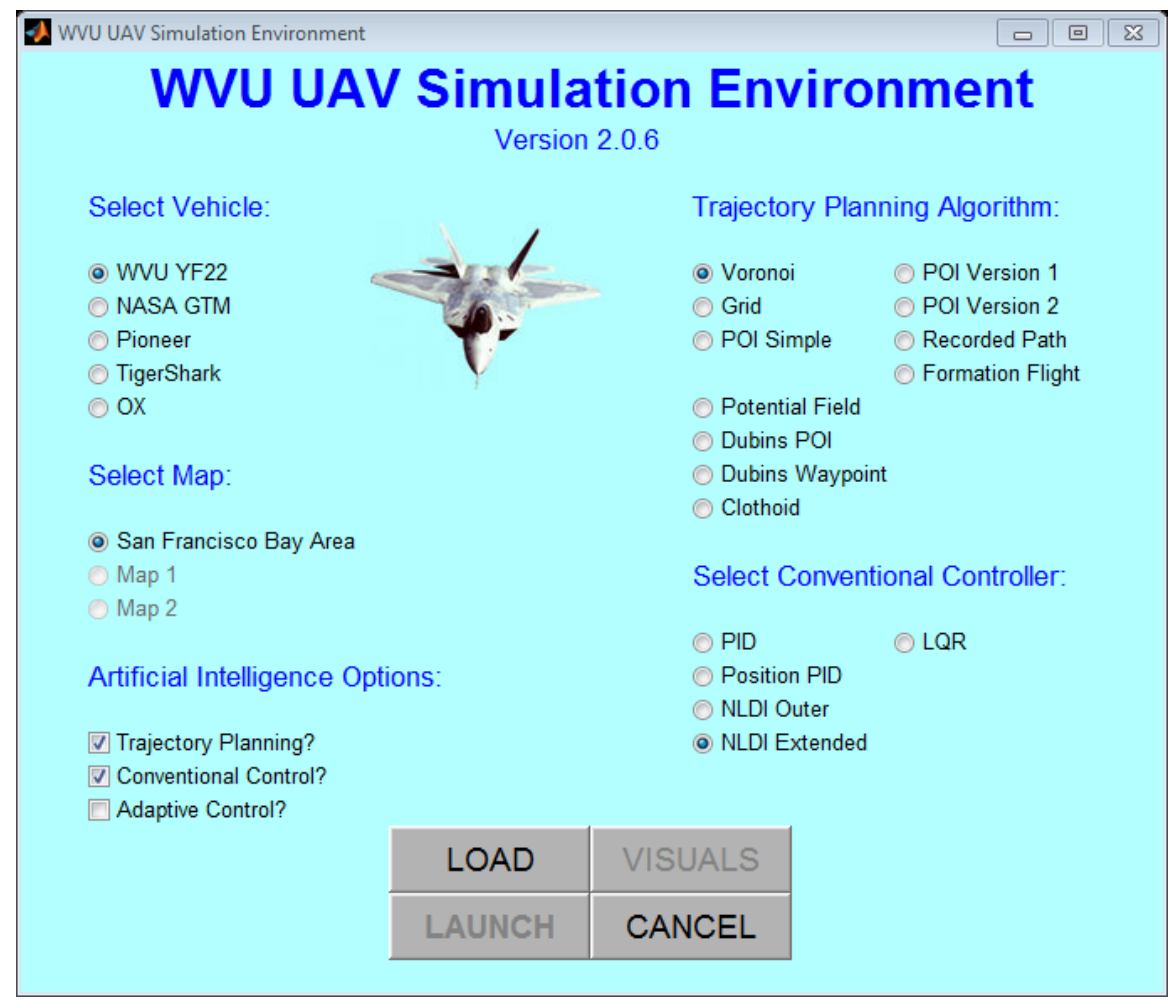

Figure 26: WVU UAV Simulation Environment GUI

On the UAVDashboard, the user can specify the initial position of the aircraft and the set of risk zones to be avoided. Alternatively only the initial position is needed if the user wishes the aircraft to flow a predefined path, which can be loaded from the Simulink model.

In addition to FlightGear and UAVDashboard as a visual feedback output, the simulation environment provides the user with plots that help to evaluate the performance of controllers under that specific scenario. These plots include the 2D trajectory, 3D trajectory, and the performance indices.

\subsection{Aircraft Dynamics}

The WVU UAV simulation environment includes five aircraft models that have various aerodynamics characteristics. These models are: WVU YF-22, NASA GTM, Pioneer, TigerShark, and OX. The WVU YF-22 research UAV was designed based on the prototype of the U.S Air Lockheed/Boeing F-22 fighter aircraft. The purpose behind designing the WVU UAV was mainly 
for testing various fault tolerant control algorithms. It is a small UAV that is powered by a miniature jet engine and has limited fuel capacity to allow only about 12 minutes of flight. This UAV is useful for testing short duration flight scenarios. It was used in this research for the purpose of analyzing the controllers' performances sensitivity to the GPS errors.

\subsection{Path Planning Algorithms}

The WVU UAV simulation environment includes several path planning algorithms. These algorithms fall into two categories: the risk zone avoidance algorithms, and the point of interest algorithms. . A variety of different approaches are implemented ranging from grid, Voronoi, and potential field methods to 2- and 3-dimensional Dubins and clothoid-based methods. For more information about these algorithms please refer to [42].

\subsection{Trajectory Tracking Algorithms}

The WVU UAV simulation environment has various trajectory tracking algorithms implemented [43-46]; however, only six different control algorithms have been chosen to investigate their fault tolerant capabilities in the presence of GPS abnormal conditions. The first three of these algorithms are conventional control systems with fixed architecture and parameters, while the last three sets are immunity-inspired adaptive control systems. Below is a brief description of each control system that has been used in this study.

\subsubsection{Conventional Algorithms}

\subsubsection{Position Proportional, Integral, and Derivative (PID) Controller}

The position PID controller works by minimizing the errors in the position between the commanded trajectory and the actual aircraft position [43]. The position PID controller calculates the position error between the desired position and the aircraft actual position (GPS reading). The outer loop controller uses this error to calculate the required bank, pitch, and thrust values needed to compensate for this error, while the inner loop controller use the bank and pitch 
angles to determine the actuator and throttle values needed to accomplish the required bank, pitch and thrust values. Figure 27 illustrate a block diagram for the position PID controller.

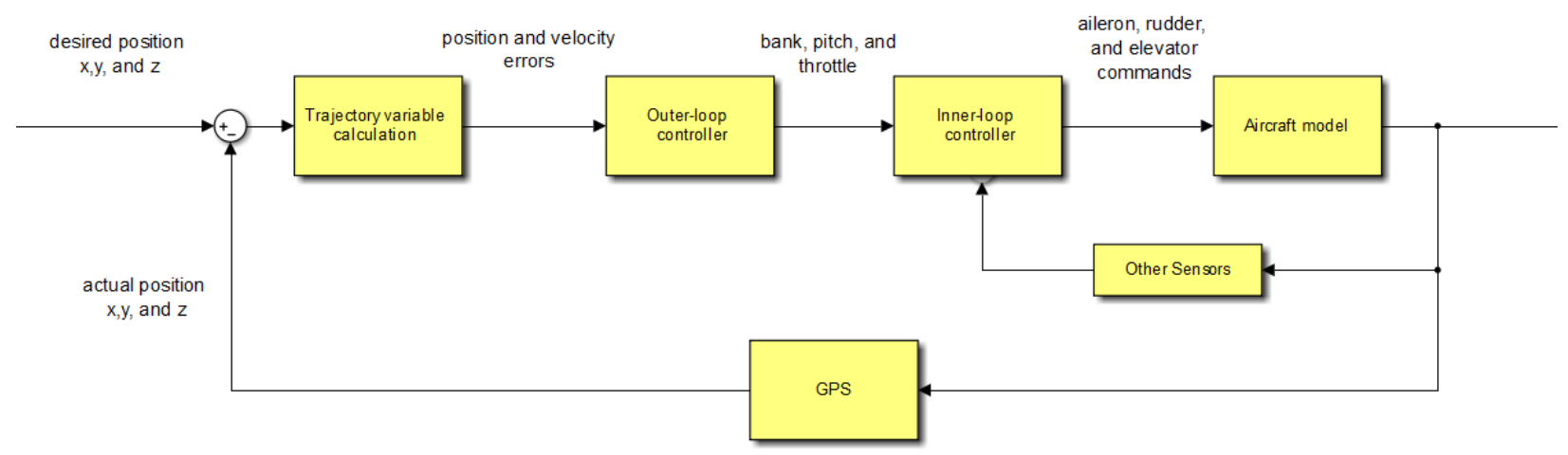

Figure 27: PID Controller Block Diagram

\subsubsection{Outer-Loop NLDI Controller}

The non-linear dynamic inversion (NLDI) outer-loop controller utilizes the inner-loop PID controller from the above described position PID controller, but replaces the outer-loop PID controller with a NLDI approach. This controller utilizes NLDI to obtain bank angle and throttle commands. The pitch angle command is still calculated by a PID controller. By utilizing a dynamic inversion approach, non-linearities in the system are cancelled out, thus resulting in a better performing controller [44].

\subsubsection{Extended NLDI Controller}

The extended NLDI controller builds upon the concept of the outer-loop NLDI controller; in the outer-loop NLDI, only the outer-most control loop is controlled by NLDI. However, in this controller, the NLDI approach has been "extended" into the inner-loop as well. This is accomplished by breaking the inner controller down into two sub-controllers. The outer-most controller in the inner loop, described as the slow-mode, uses bank angle and pitch angle commands to command pitch, roll, and yaw rate commands. These commands are used as inputs by the fast-mode controller, which produces the control surface commands for the ailerons, elevators, and rudder[45].

\subsubsection{Adaptive Algorithms}


As opposed to the conventional controllers, which have constant gains, adaptive controllers have gains that are adjusted during flight to cope with changing conditions [46]. The approach is expected to allow for better performance and increased fault tolerant capability. Both position PID and outer loop NLDI controllers use the adaptive gains to scale the change in Euler angle error to produce a control response proportional with the value of that rate. Extended NLDI controller uses the adaptive gain to scale the angular rate instead of using Euler angle to produce corresponding control response. It should be noted that these algorithm have been designed with the primary objective of improving fault tolerant capabilities in the presence of actuator failures

\subsection{Performance Metrics}

The overall performance of a controller will be defined using two performance criteria [47]. The first criterion is based on the trajectory tracking accuracy and is supported by a set of trajectory tracking indices. These indices are expected to capture the performance of a controller in terms of its capability to track the trajectory with as minimum errors as possible. The second criterion is based on the amount of control actuation and is supported by a set of control activity indices which attempt to assess performance in terms of the controller's ability to keep track of the trajectory with as minimum commands as possible, and without reaching surface saturation.

\subsubsection{Trajectory Tracking Indices}

The trajectory tracking performance is evaluated using maximum, mean, and standard deviation of the trajectory tracking errors in the horizontal XY plane, along the vertical Z-axis, and in the 3dimensional space. Let $[x(t), y(t), z(t)]$ be the commanded trajectory point at time $t$, and $\left[x_{a}(t) y_{a}(t) z_{a}(t)\right]$ the actual aircraft position at time $t$, then the horizontal xy plane tracking error is defined as:

$$
x y_{\text {error }}=\sqrt{\left[x_{a}(t)-x(t)\right]^{2}+\left[y_{a}(t)-y(t)\right]^{2}}
$$

and the vertical $Z$ direction tracking error is defined as:

$$
z_{\text {error }}=\sqrt{\left[z_{a}(t)-z(t)\right]^{2}}
$$

while the three dimensional tracking error is defined as: 


$$
x y z_{\text {error }}=\sqrt{\left[x_{a}(t)-x(t)\right]^{2}+\left[y_{a}(t)-y(t)\right]^{2}+\left[z_{a}(t)-z(t)\right]^{2}}
$$

Then for the total simulation time or for a pre-defined length of time, all the nine trajectory tracking indices can be defined as follows.

The horizontal $x y$ plane maximum tracking error:

$$
x y_{\text {max error }}=\max \left(\left|x y_{\text {error }}\right|\right)
$$

The vertical maximum tracking error:

$$
z_{\text {max error }}=\max \left(\left|z_{\text {error }}\right|\right)
$$

The maximum three dimensional tracking error:

$$
x y z_{\text {max error }}=\max \left(\left|x y z_{\text {error }}\right|\right)
$$

The horizontal $x y$ plane average tracking error:

$$
x y_{\text {Ave error }}=\operatorname{mean}\left(\left|x y_{\text {error }}\right|\right)
$$

The vertical average tracking error:

$$
z_{\text {Ave error }}=\operatorname{mean}\left(\left|z_{\text {error }}\right|\right)
$$

The average 3-dimensional tracking error:

$$
x y z_{\text {Ave error }}=\operatorname{mean}\left(\left|x y z_{\text {error }}\right|\right)
$$

The standard deviation of horizontal xy tracking error

$$
x y_{\text {error Std }}=\operatorname{STD}\left(\left|x y_{\text {error }}\right|\right)
$$

The standard deviation of vertical tracking error

$$
\boldsymbol{z}_{\text {error std }}=\operatorname{STD}\left(\left|z_{\text {error }}\right|\right)
$$

The standard deviation of the 3-dimensional tracking error

$$
x y z_{\text {error Std }}=\operatorname{STD}\left(\left|x y z_{\text {error }}\right|\right)
$$

\subsubsection{Control Activity Indices}

The control activity indices are defined using two primary parameters: the integral of the absolute value of the rate of change was used to best capture the controller activity, and the saturation percentage for stabilator, aileron, rudder, and throttle. Let $\boldsymbol{\delta}_{\boldsymbol{e}}, \boldsymbol{\delta}_{\boldsymbol{a}}, \boldsymbol{\delta}_{r}$, and $\boldsymbol{\delta}_{\boldsymbol{t}}$ be the commanded deflections of the stabilator, aileron, rudder, and throttle respectively, then the control activity indices are defined as [47]: 
Integral of stabilator deflection rate of change

$$
I \dot{\delta}_{e}=\frac{1}{T} \int_{0}^{T}\left|\dot{\delta}_{e}(t)\right| d t
$$

Integral of aileron deflection rate of change

$$
I \dot{\delta}_{a}=\frac{1}{T} \int_{0}^{T}\left|\dot{\delta}_{a}(t)\right| d t
$$

Integral of rudder deflection rate of change

$$
I \dot{\delta}_{r}=\frac{1}{T} \int_{0}^{T}\left|\dot{\delta}_{r}(t)\right| d t
$$

Integral of the throttle command rate of change

$$
I \dot{\delta}_{t}=\frac{1}{T} \int_{0}^{T}\left|\dot{\delta}_{t}(t)\right| d t
$$

The stabilator saturation index is defined as:

$$
S_{\delta e}=\frac{100}{T} \int_{0}^{T}\left(\tilde{\delta}_{e 1}(t)+\tilde{\delta}_{e 2}(t)\right) d t
$$

where

$$
\tilde{\delta}_{e 1}=\left\{\begin{array}{l}
0 \text { for } \delta_{e}<\delta_{e \max } \\
1 \text { for } \delta_{e} \geq \delta_{e \max }
\end{array}\right.
$$

and

$$
\tilde{\delta}_{e 2}=\left\{\begin{array}{l}
0 \text { for } \delta_{e}>\delta_{e \min } \\
1 \text { for } \delta_{e} \leq \delta_{e \min }
\end{array}\right.
$$

The aileron saturation index is defined as:

$$
S_{\delta a}=\frac{100}{T} \int_{0}^{T} \tilde{\delta}_{a}(t) d t
$$

where

$$
\tilde{\delta}_{a}=\left\{\begin{array}{l}
0 \text { for } S_{a}<S_{a \max } \\
1 \text { for } S_{a} \geq S_{a \max }
\end{array}\right.
$$

The rudder saturation index is defined as:

$$
S_{\delta r}=\frac{100}{T} \int_{0}^{T} \tilde{\delta}_{r}(t) d t
$$


where

$$
\tilde{\delta}_{r}=\left\{\begin{array}{l}
0 \text { for } \delta_{r}<\delta_{r \text { max }} \\
1 \text { for } \delta_{r} \geq \delta_{r \text { max }}
\end{array}\right.
$$

Finally, the throttle saturation index is defined as:

$$
S_{\delta t}=\frac{100}{T} \int_{0}^{T} \tilde{\delta}_{t}(t) d t
$$

where

$$
\tilde{\delta}_{t}=\left\{\begin{array}{l}
0 \text { for } \delta_{t}<\delta_{t \max } \\
1 \text { for } \delta_{t} \geq \delta_{t \text { max }}
\end{array}\right.
$$

\subsubsection{The Total Performance Index (PI)}

The trajectory tracking indices along with the control activity indices capture all the trajectory tracking performance characteristic of a controller; however, it will be difficult to compare all these 17 indices for one or more controller(s) under different flight conditions. Each set of the trajectory tracking indices and the control activity indices were reduced to the overall trajectory tracking index and the overall control activity index by means of a normalization and weighted sum process. The normalization values for each 17 indices were chosen based on the performance of all the controllers, while the weights of each index were chosen based on the importance of that index toward the total performance index.

The total trajectory tracking index (TTI) has a value between zero and one. A trajectory tracking index of 1 means that the controller has the best trajectory tracking capabilities and has zero tracking error, while a value of zero to the total trajectory index means that the controller is having large errors that exceed all normalization limits. The same approach is applied to the total control activity index (CAI). A value of 1 means that the controller is achieving the task with a small amount of controller activity; however, a value of zero shows that the controller is having large control activities with surfaces possibly reaching saturation.

The total performance index (PI) was calculated by two methods: the constant weight method and the variable weight method. In the constant weight method, the trajectory tracking error and the control activity index were calculated for the full length of the path, then a set of constant weights was selected to sum the overall trajectory tracking and the overall control activity index 
into the total performance index. The set of weights used were 0.7 for the trajectory tracking index and 0.3 for the control activity index based on our interest in having more accurate path tracking ability. In the second method, the path was divided into different segments based on whether the aircraft was flying in straight line or turning. In the straight path segments, more weight was giving to the control activity index to penalize the controllers for having controller action in a straight path flight, while a higher weight was given to the trajectory tracking in the turning segments to make sure that the aircraft is following the trajectory in these critical segments. The set of weights used were 0.7 for the control activity index and 0.3 for the trajectory index in the straight segments, while 0.3 was given to the control activity index and 0.7 to the trajectory tracking index in the turning segments.

For the given weights used in the fixed weight algorithm, a PI above 0.6 generally provide "good" tracking with little deviation from the commanded path and few control oscillations. Performance indices in the range of 0.3 to 0.6 provide worse tracking, with the aircraft occasionally getting lost from the path. Performance indices below 0.3 generally result in the aircraft almost immediately getting lost or crashing into the ground.

Whether using the fixed or the variable weight algorithms, the total performance index will give the controller a performance indication with respect to the algorithm criteria. For example, using the variable weight index on all curves trajectory will give the same result as using fixed weight algorithm; however, if the path was composed of straight and curves then we would have different results. It should be noted that the performance index must be designed carefully to accurately capture and reflect the objective of the user and the mission. Any change in the algorithms weights, normalization values, or even any change in the path is likely to change the performance evaluation results and possibly the controllers' ranking.

For the given weights used in variable weight algorithm, a PI above 0.7 generally provides "good" tracking with little deviation from the commanded path and few control oscillations. Performance indices in the range of 0.4 to 0.7 provide worse tracking, with the aircraft occasionally getting lost from the path. Performance indices below 0.4 results in the aircraft almost immediately getting lost or crashing into the ground. 
An example of a "good" trajectory tracking performance and its corresponding controller surface deflection in provided in Figure 28 and Figure 29 respectively. This was achieved by the position PID controller under the presence of the GPS error model reference parameter defined in next section. The total variable weight $\mathrm{PI}$ recorded was 0.87 with $\mathrm{TTI}=0.78$ and $\mathrm{CAI}=0.9$.

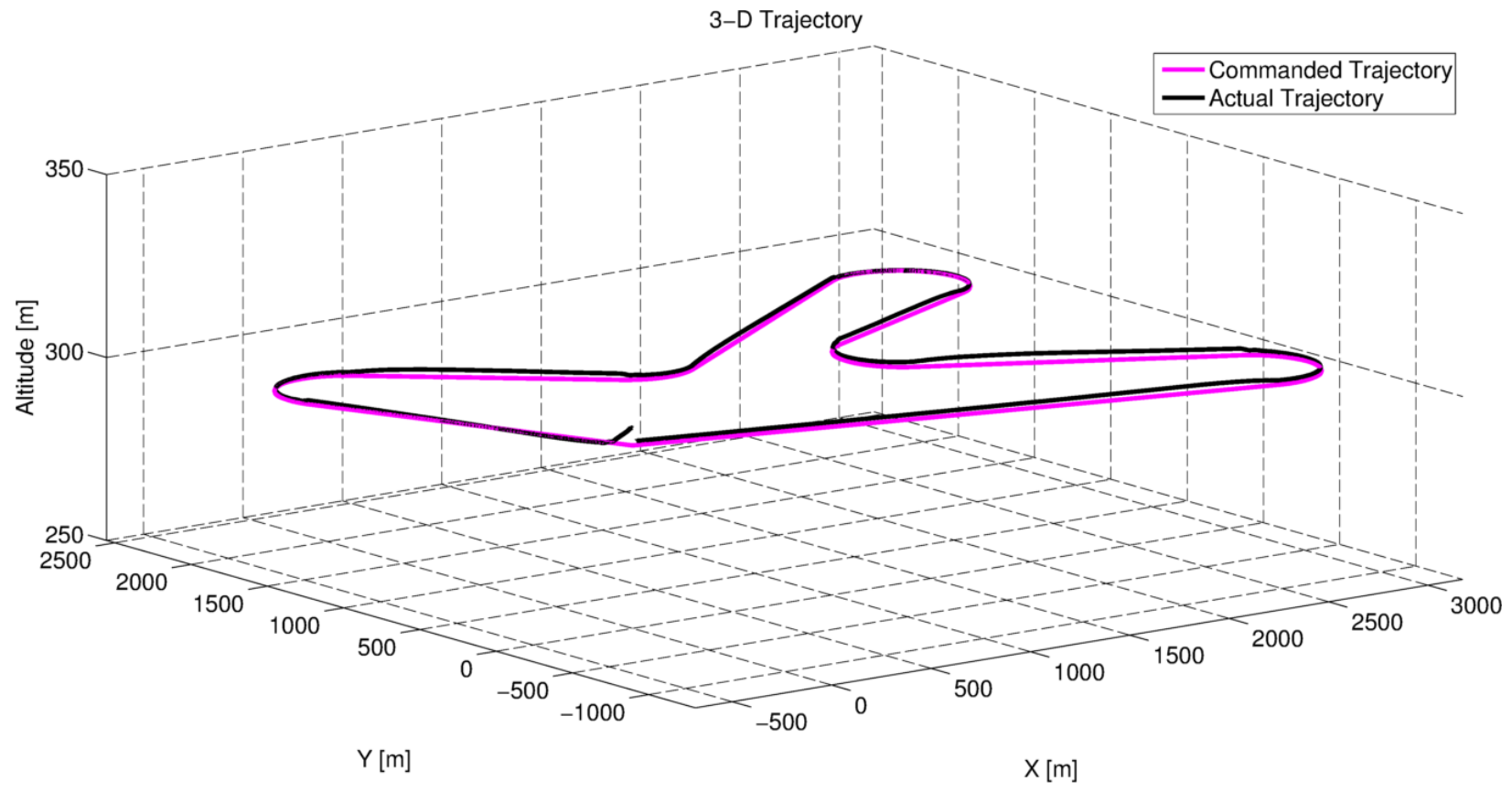

Figure 28: Actual and Commanded Trajectory for a Controller with "Good" Performance Index
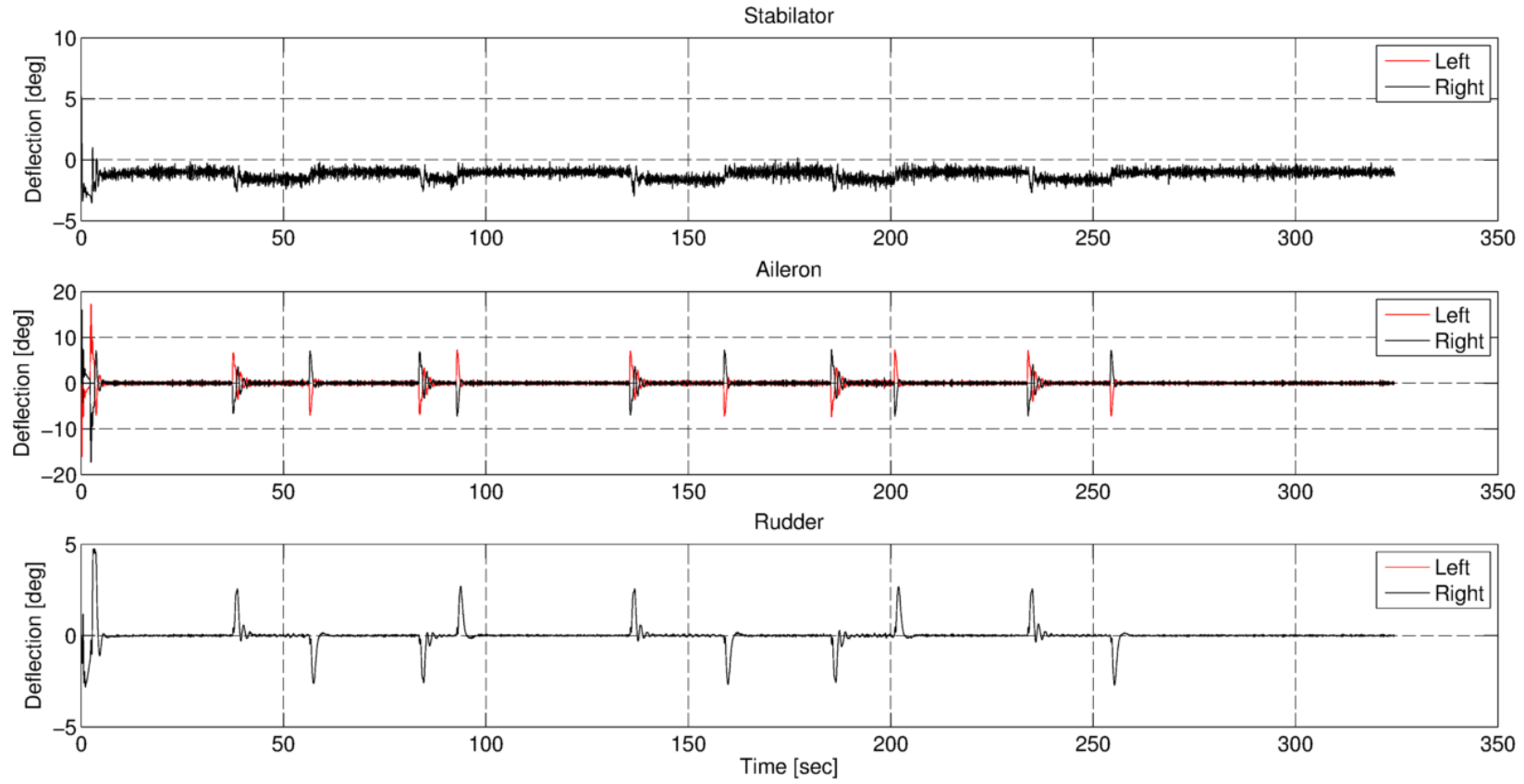

Figure 29: Controller Surface Deflection for a Controller with "Good" Performance Index 
Figure 30 and Figure 31 present an example of actual aircraft trajectory and its corresponding controller surface deflections for a controller that has an "adequate" total variable weight performance index $\mathrm{PI}=0.56$. The specific contribution to the total $\mathrm{PI}$ are: $\mathrm{TTI}=0.5$, and $\mathrm{CAI}=$ 0.58 .

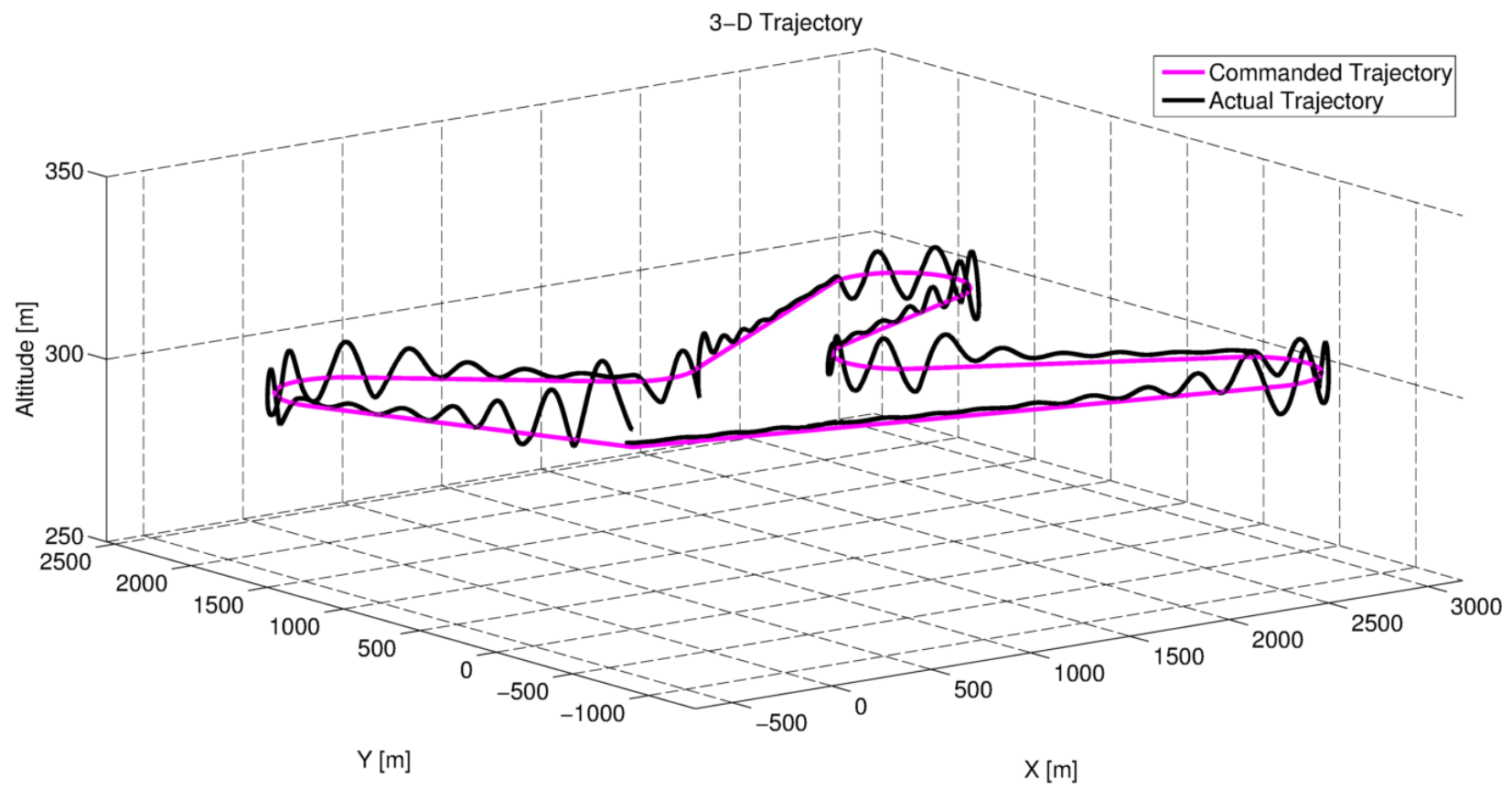

Figure 30: Actual and Commanded Trajectory for a Controller with "Adequate" Performance Index
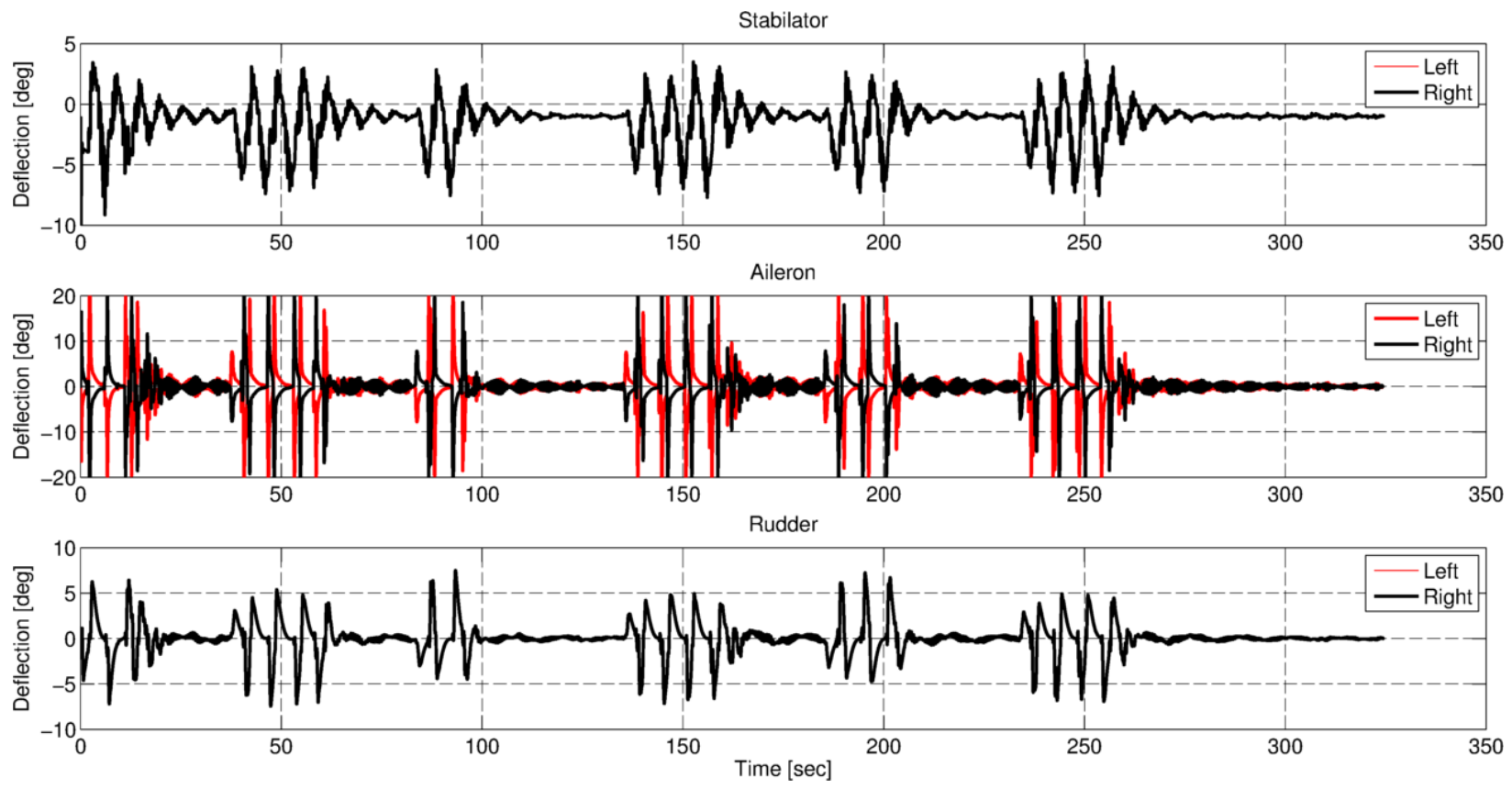

Figure 31: Controller Surface Deflection for a Controller with “Adequate" Performance Index 
Finally, an example of "poor" performance is presented in Figure 32 and Figure 33. The controller has a variable weight performance index $=0.2$, with specific contributions of TTI $=$ 0.22 , and $\mathrm{CAI}=0.18$.

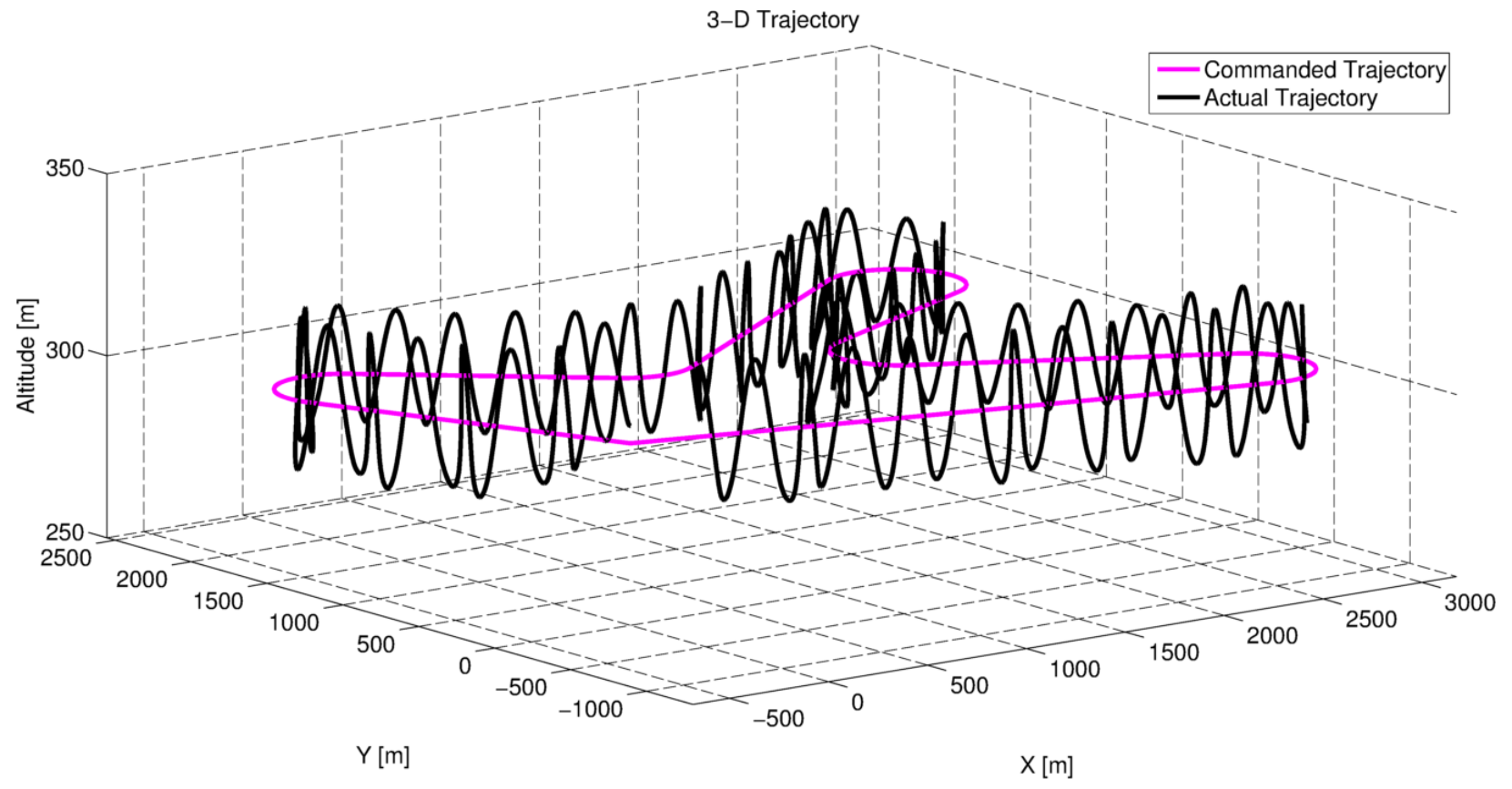

Figure 32: Actual and Commanded Trajectory for a Controller with “Poor" Performance Index
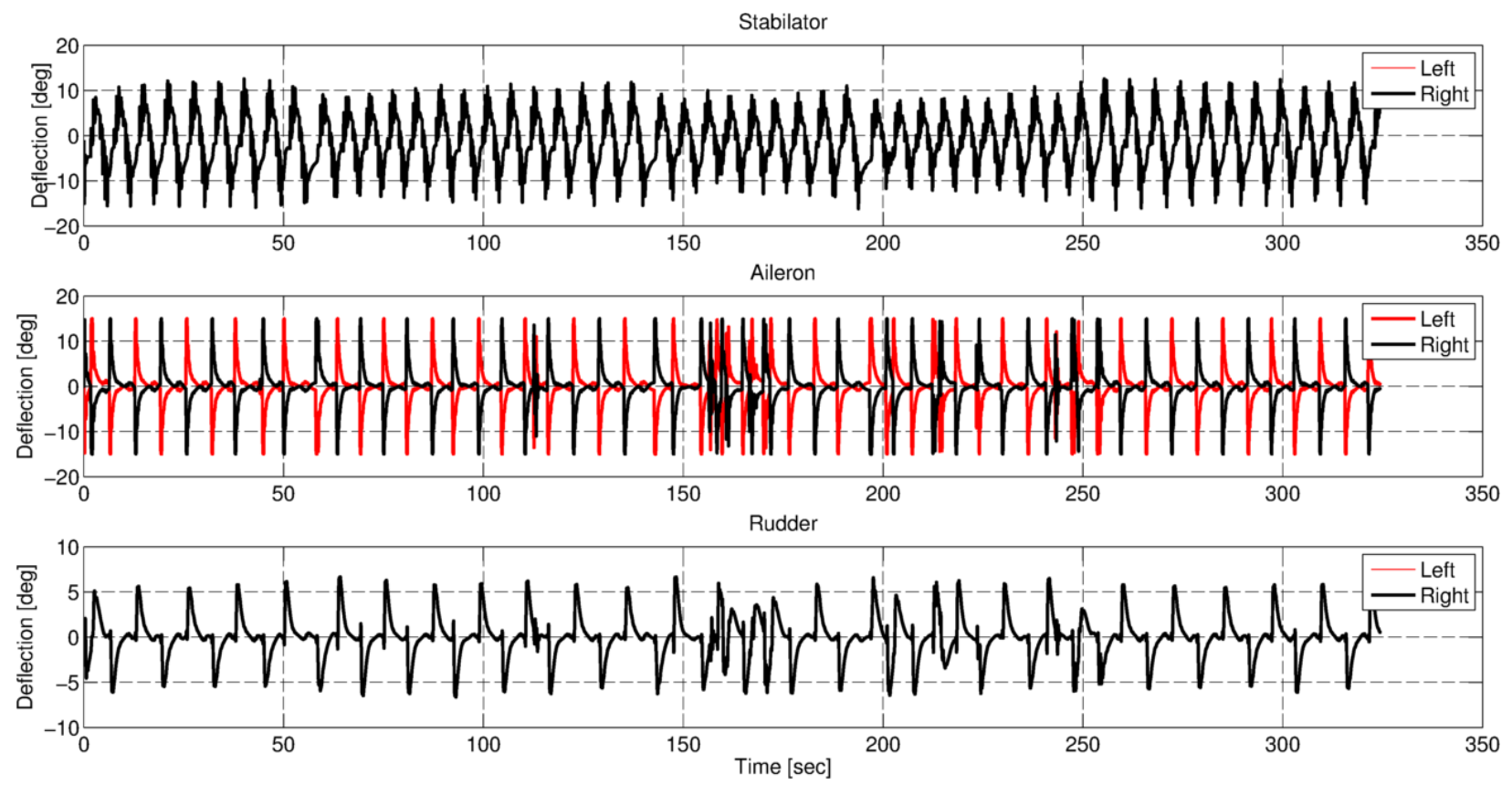

Figure 33: Controller Surface Deflection for a Controller with "Poor" Performance Index 


\section{Analysis and Results}

This chapter provides a trajectory tracking controller performance sensitivity analysis for normal and abnormal position and velocity GPS measurements. This analysis was performed in the WVU UAV simulation environment using the WVU YF22 aircraft model. The aircraft speed was constant and equal to 40 meter per second during the simulation; however, the control has the ability to change the aircraft speed, if needed. No wind, turbulence, or actuator failure where considered in these analyses. Individual parameters were varied in the GPS reference model to study their effects on the trajectory tracking performance of the controllers. The reference model for the GPS at nominal conditions can be found below in Table 9.

\begin{tabular}{|c|c|}
\hline GPS Error Model Parameter & Reference Value \\
\hline Position Update Rate & 0.02 seconds \\
\hline Velocity Update Rate & 0.02 seconds \\
\hline Time Delay & 0.04 seconds \\
\hline Position Error STD & 0.05 meters \\
\hline Velocity to Position Error STD Ratio & 0.3 \\
\hline Position Bias & 2 meters (maximum) \\
\hline HDOP & 1.5 (maximum) \\
\hline VDOP & 2.5 (maximum) \\
\hline Failures & None \\
\hline
\end{tabular}

Table 9: GPS Error Model Reference Parameters

A summary of the experiments performed is given below in Table 10. The main parameter column represents the GPS error model parameter that was varied in that particular set of tests. The range column represents the range and the increment size of the primary variable parameter. For the range, the first number provides the starting value, the second number provides the increment size, and the last number provides the final value. The secondary parameter represents any additional parameters which were varied in that analysis. The vector notation in that column means the analysis was perform $n$ times with one value of this vector each time, while the range, step and end notation means it was varied the same time with the 
main parameters. Finally, the test No. column represents the number of test that were performed for that particular analysis.

\begin{tabular}{|c|c|c|c|c|}
\hline $\begin{array}{c}\text { Main } \\
\text { Parameter }\end{array}$ & Range & $\begin{array}{c}\text { Sec. } \\
\text { Parameter }\end{array}$ & Value & Tests No. \\
\hline \multicolumn{5}{|c|}{ Normal Operation } \\
\hline Time delay & 0.02:0.04:0.98 & & & 25 \\
\hline $\begin{array}{c}\text { Position } \\
\text { Update } \\
\text { Interval (sec) }\end{array}$ & 0.02:0.04:0.98 & $\begin{array}{c}\text { Velocity } \\
\text { Update } \\
\text { Interval (sec) }\end{array}$ & 0.02:0.04:0.98 & 25 \\
\hline $\begin{array}{c}\text { Velocity } \\
\text { Update } \\
\text { Interval (sec) }\end{array}$ & 0.02:0.08:0.98 & $\begin{array}{c}\text { Position } \\
\text { Update } \\
\text { Interval (sec) }\end{array}$ & 0.02 & 13 \\
\hline $\begin{array}{l}\text { Position Error } \\
\text { STD }(\mathrm{m})\end{array}$ & $0: 0.25: 4$ & $\begin{array}{l}\text { Velocity Error } \\
\text { STD }(\mathrm{m} / \mathrm{s})\end{array}$ & $\begin{array}{c}{[00.30 .651]^{*} \text { Position }} \\
\text { Error STD }\end{array}$ & 64 \\
\hline $\begin{array}{l}\text { Velocity Error } \\
\text { STD }(\mathrm{m} / \mathrm{sec})\end{array}$ & $0: 0.25: 4$ & $\begin{array}{l}\text { Position Error } \\
\text { STD }(\mathrm{m} / \mathrm{s})\end{array}$ & 0.05 & 16 \\
\hline \multicolumn{5}{|c|}{ Failure Operations } \\
\hline $\begin{array}{l}\text { Measurement } \\
\text { Noise }(\mathrm{m})\end{array}$ & {$\left[\begin{array}{lllll}2 & 4 & 6 & 8 & 10\end{array}\right]$} & $\begin{array}{l}\text { Noise duration } \\
\text { (sec) }\end{array}$ & {$\left[\begin{array}{llll}12 & 25 & 50 & 75\end{array}\right]$} & 20 \\
\hline $\begin{array}{l}\text { Measurement } \\
\text { Step }(\mathrm{m})\end{array}$ & {$\left[\begin{array}{llllll}10 & 25 & 50 & 75 & 100\end{array}\right]$} & $\begin{array}{c}\text { Step duration } \\
\text { (sec) }\end{array}$ & {$\left[\begin{array}{llll}12 & 25 & 50 & 75\end{array}\right]$} & 20 \\
\hline $\begin{array}{l}\text { Measurement } \\
\operatorname{Ramp}(\mathrm{m} / \mathrm{s})\end{array}$ & {$\left[\begin{array}{llllll}0.6 & 1.5 & 2.5 & 5 & 10\end{array}\right]$} & $\begin{array}{l}\text { Ramp duration } \\
\text { (sec) }\end{array}$ & {$\left[\begin{array}{llll}12 & 25 & 50 & 75\end{array}\right]$} & 20 \\
\hline $\begin{array}{l}\text { Measurement } \\
\text { Sine }(\mathrm{m})\end{array}$ & {$\left[\begin{array}{llllll}10 & 25 & 50 & 75 & 100\end{array}\right]$} & $\begin{array}{c}\text { Sine duration } \\
(\mathrm{sec})\end{array}$ & {$\left[\begin{array}{llll}12 & 25 & 50 & 75\end{array}\right]$} & 20 \\
\hline
\end{tabular}

Table 10: Analysis Summary

\subsection{Controllers' Performance vs. Path}

To study the effects of path complexity on the trajectory tracking performance under the presence of GPS abnormal conditions, the tests summarized above in Table 10 were performed using two different paths of different relative complexity. The first path, shown in Figure 34 below, is composed of straight and circular path segments at a constant altitude of 300 meters which is a typical trajectory for the small UAV considered. This path was designed to have all possible left, right, and maximum bank angle turns to cover all the aircraft turnings scenarios; however, the length of this path was short due to time constraints. The second path is composed of ascending and descending turns in the shape of letter S. Figure 35 and Figure 36 provide 2 and 3 dimensional representations of this path, respectively. Qualitatively speaking, the 3 
dimensional nature of the second path makes it more difficult for the trajectory tracking controllers to follow.

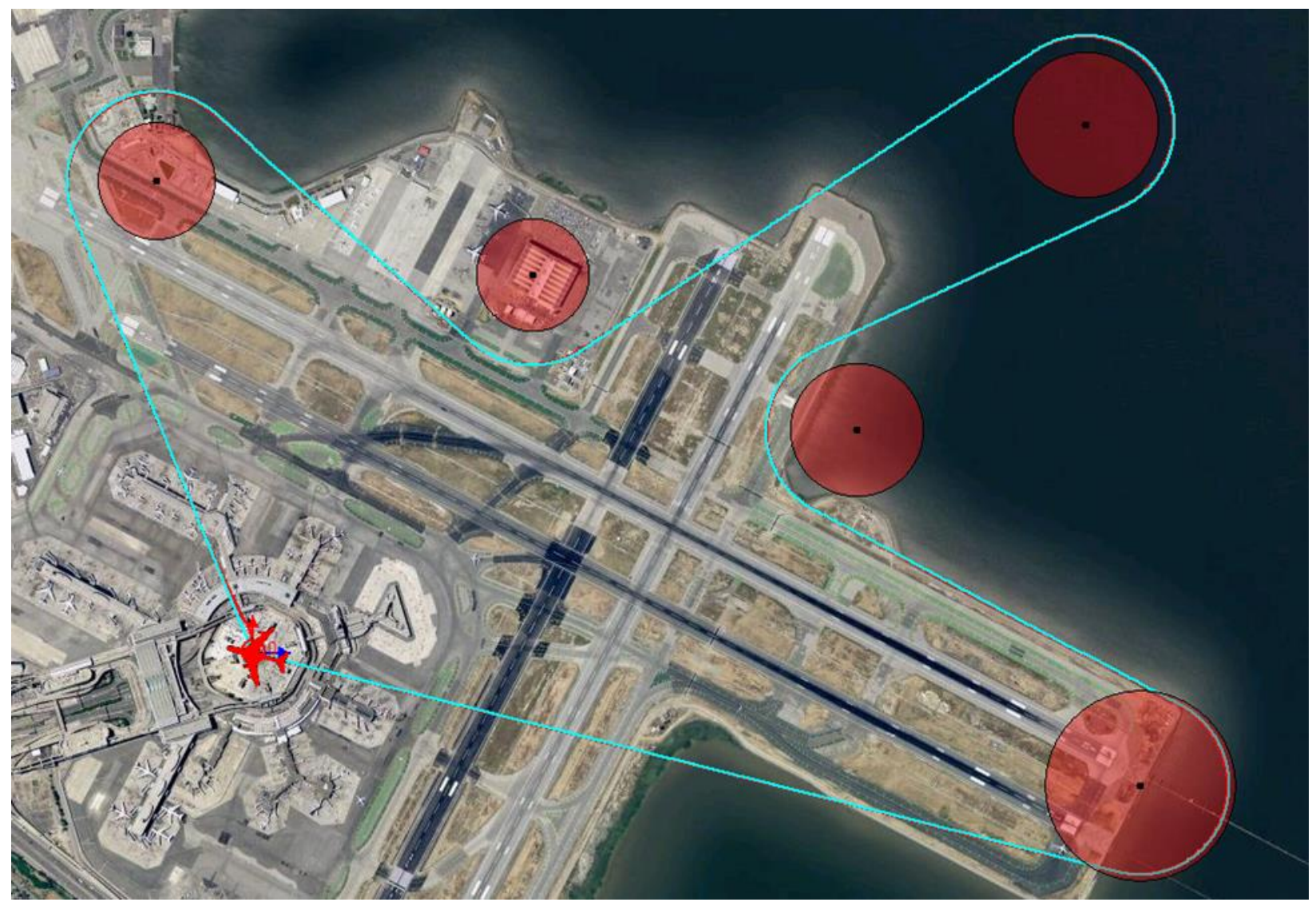

Figure 34: Test Path No.1

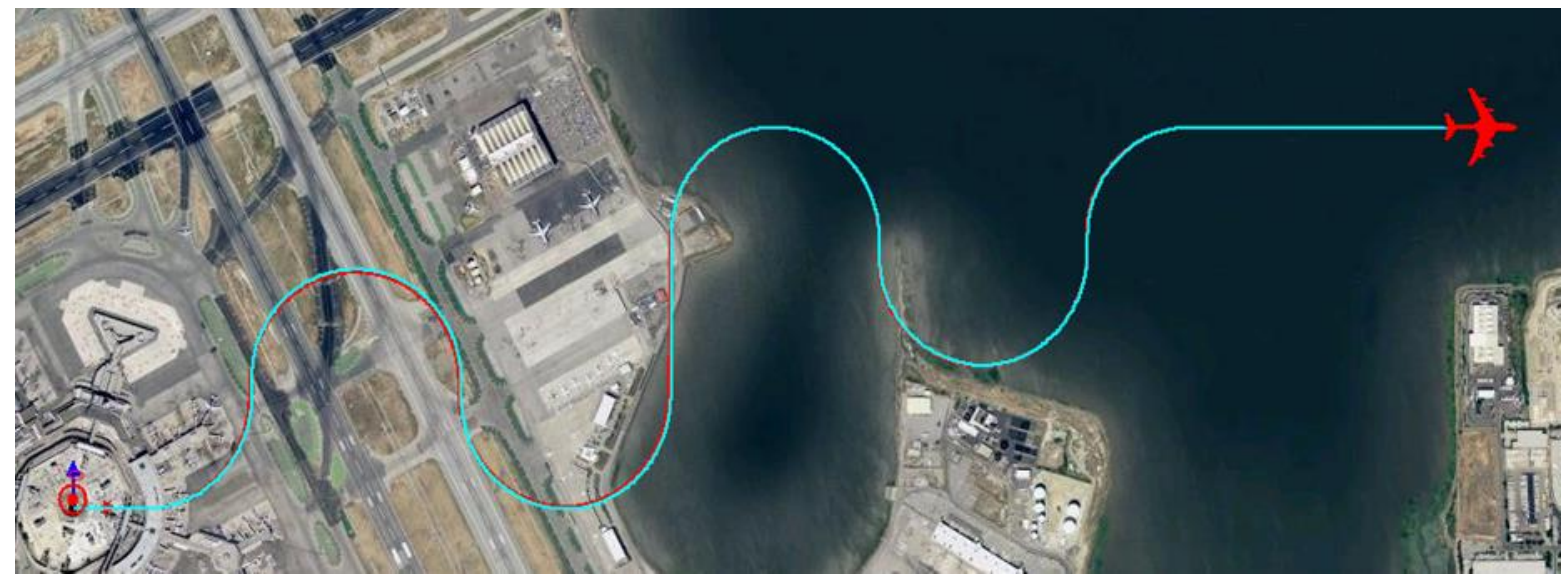

Figure 35: Test Path No.2 in 2D 


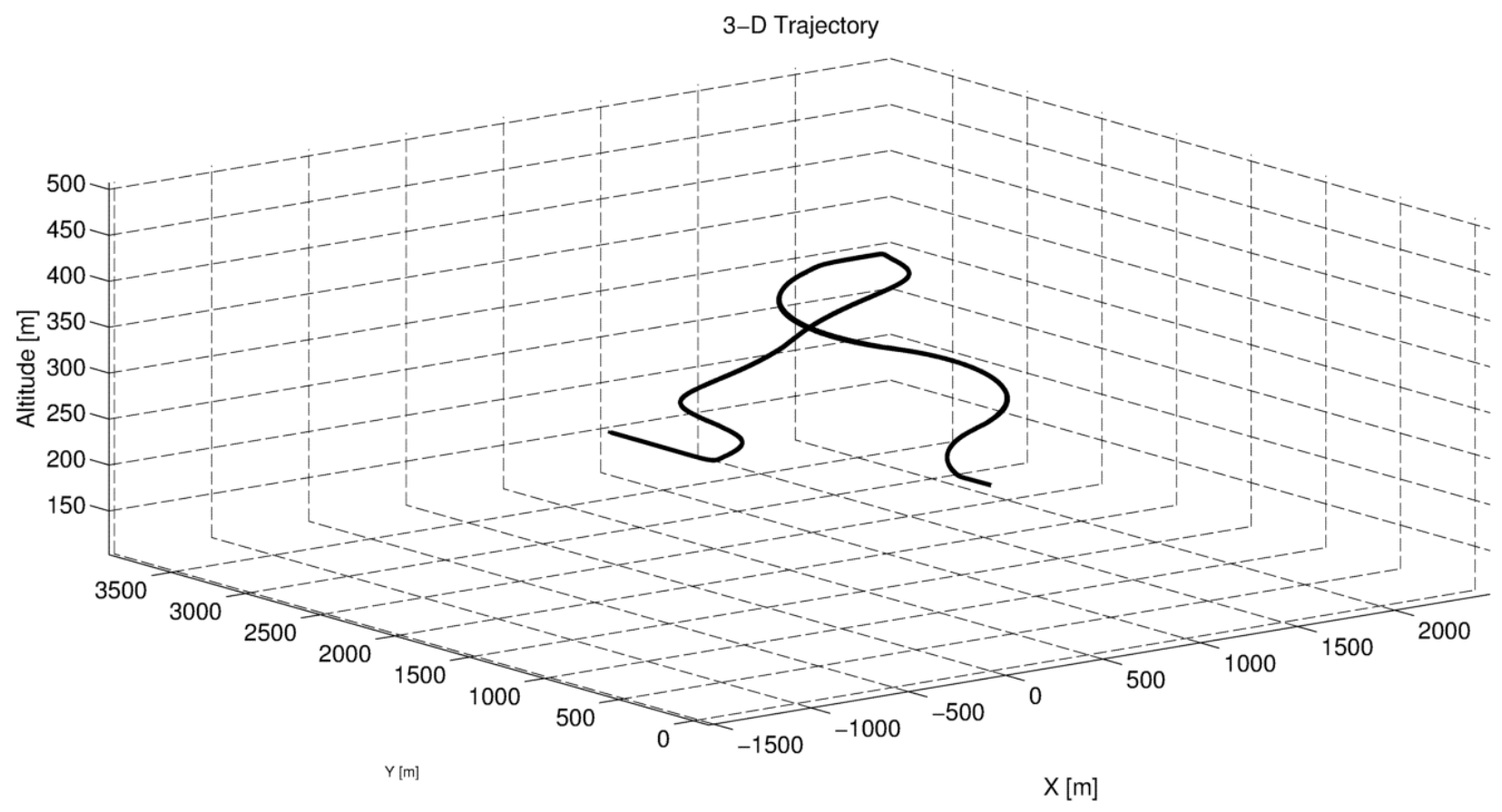

Figure 36: Test Path No.2 in 3D

In general, similar performance trends were observed in the results for both paths. However, a few tests showed more pronounced performance variations between the two paths. For example, when subjected to GPS time delays, the performance of the trajectory tracking controllers gradually decreases with the simpler, two dimensional path (see Figure 37), whereas a sharp performance decline is observed around a time delay of $0.60 \mathrm{sec}$ for the more complex three dimensional path (see Figure 38).

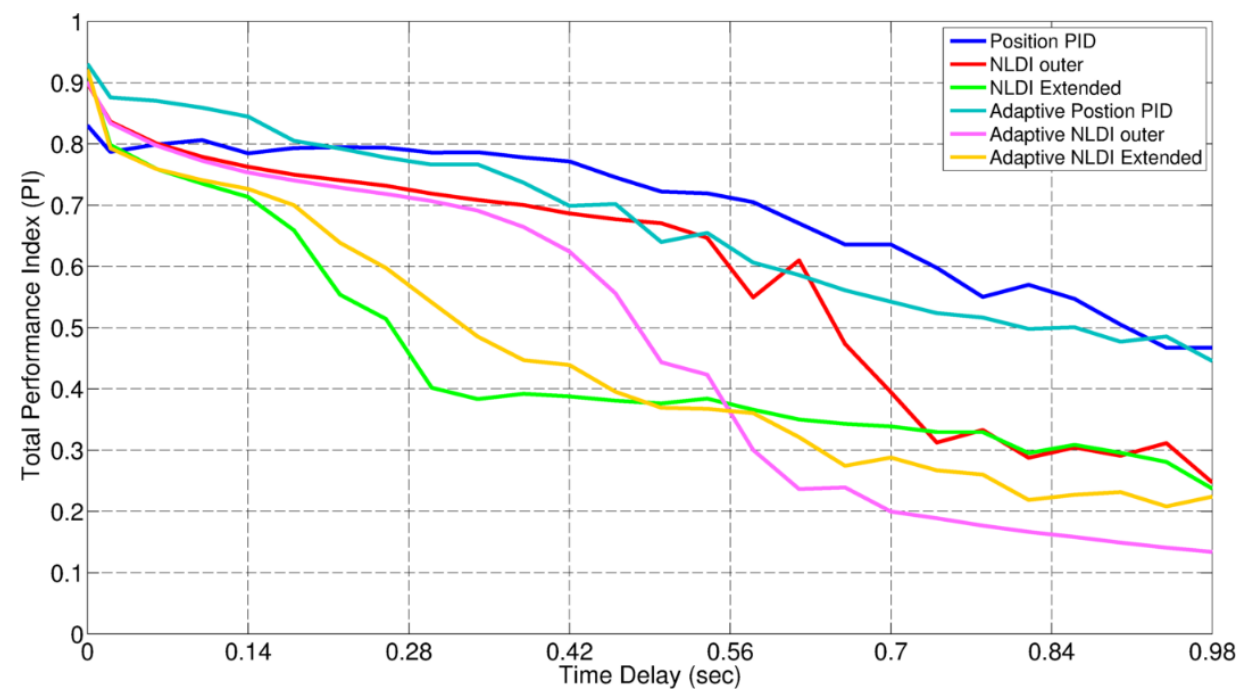

Figure 37: Controllers' PI vs. Time Delay (Path No.1 Analysis) 


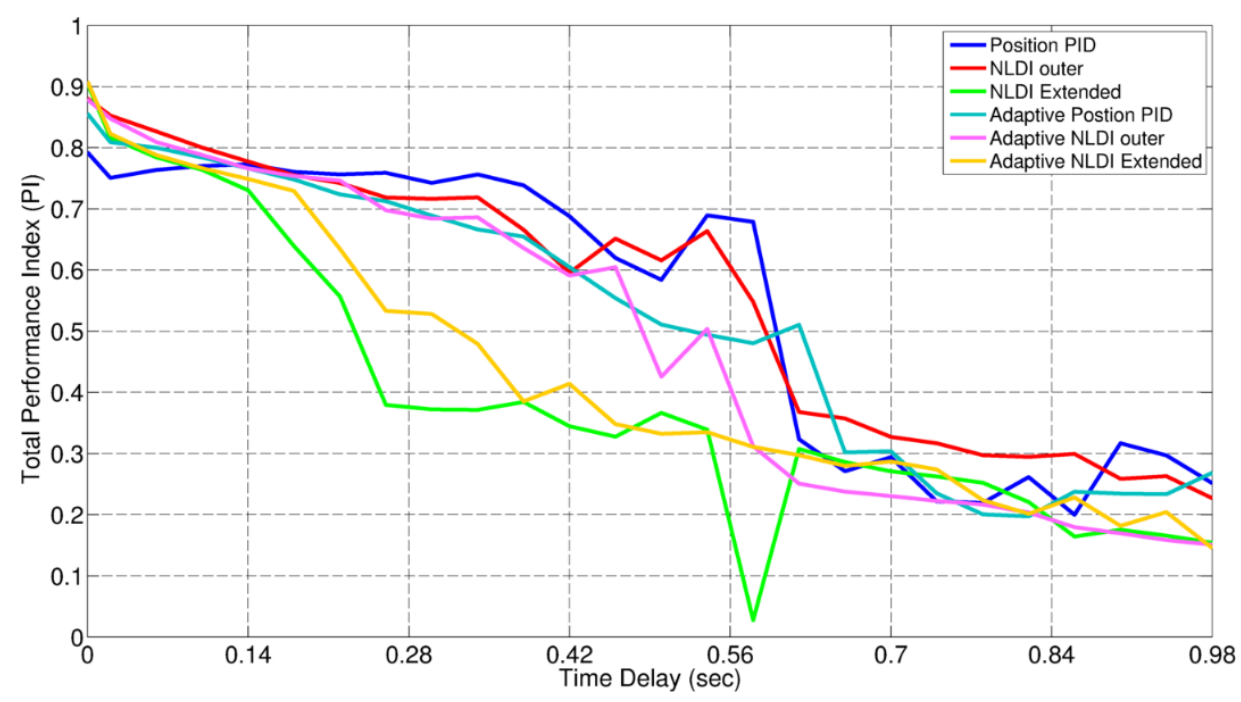

Figure 38: Controllers' PI vs. Time Delay (Path No.2 Analysis)

The position and velocity update interval analysis shows a minor difference between the two paths. The first path analysis (Figure 39) shows the NLDI extended controller to perform better than its adaptive counterpart for update intervals larger than 0.56 seconds, while the second path analysis (Figure 40) shows the reversed trend. However, the controller's ranking and its performance is the same on both cases.

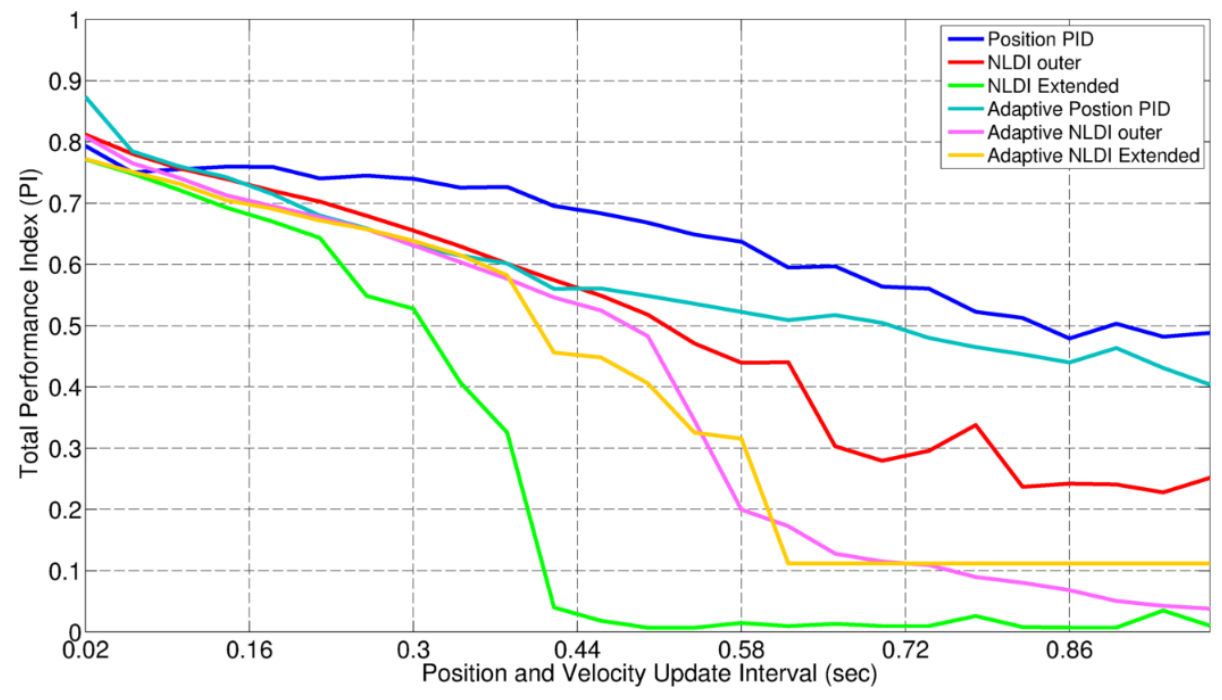

Figure 39: Controllers' PI vs. Update Interval (Path No.1 Analysis) 


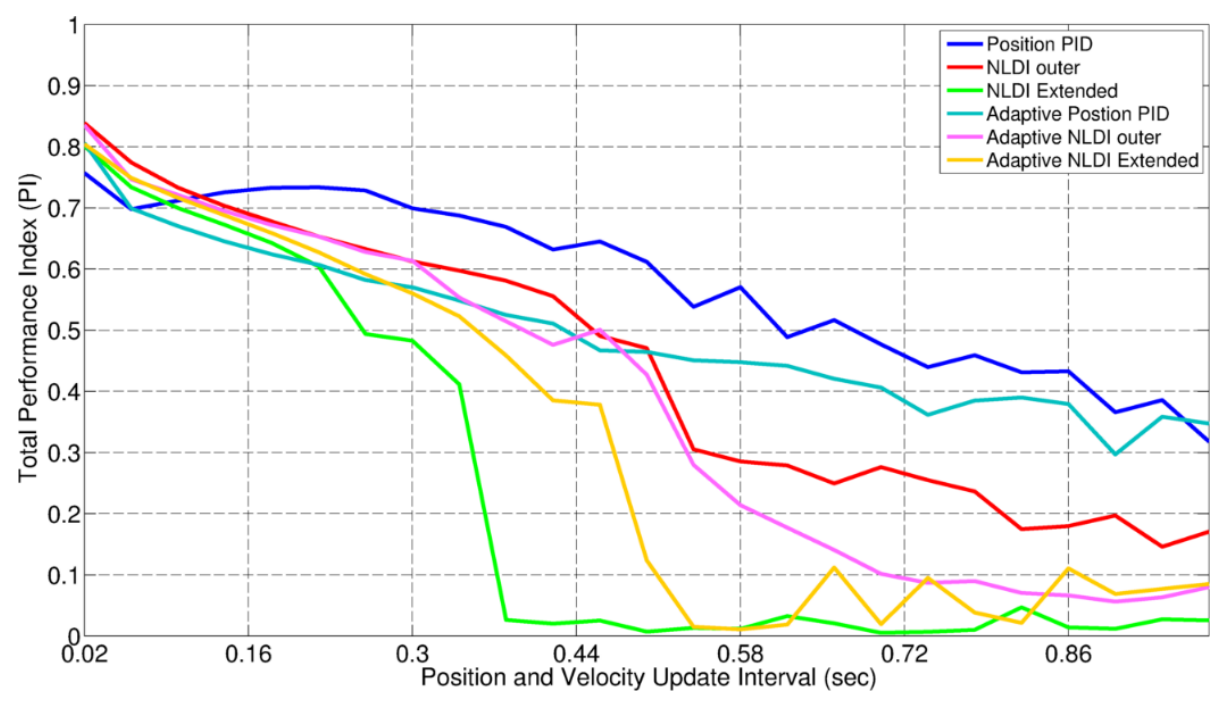

Figure 40: Controllers' PI vs. Update Interval Analysis (Path No.2 Analysis)

\subsection{Variable Weight vs. Fixed Weight Performance Algorithm}

The trajectory tracking performance index calculation algorithm is used to evaluate the performance of each controller based on a set of criteria. The variable weight algorithm evaluates the controller performance of straight path segments and turns separately. On straight path sections, this algorithm will penalize the controller for having additional unnecessary control surface activity, while giving more allowance for trajectory error. On the other hand, this algorithm will more severely penalize trajectory tracking errors on curves, while allowing more controller activity. The variable weight algorithm was used in the normal operation analysis because it is logically acceptable. The $y$-axis is labeled "Total Variable Weight Performance Index" wherever the algorithm was used. The fixed weight algorithm has different performance calculation criteria. This algorithm places more emphasis on maintaining low trajectory tracking error than control surface activity. This algorithm is used in the abnormal condition analysis section, because it provides a good indication about which controller could minimize the failure effect even with having extra controller activity. The $y$-axis is titled "Total Performance Index" wherever the algorithm was used.

A direct comparison between these two algorithms might be invalid since they have different objectives; however, a closer look at one case where the performance was evaluated using both algorithms will help better understand the similarities and differences between the two metrics. Figure 41 illustrates the controllers' trajectory tracking performance in the presence of position 
and velocity noise calculated using the variable weight algorithm, while Figure 42 shows the controllers' PI calculated using the fixed weight algorithm for the same analysis.

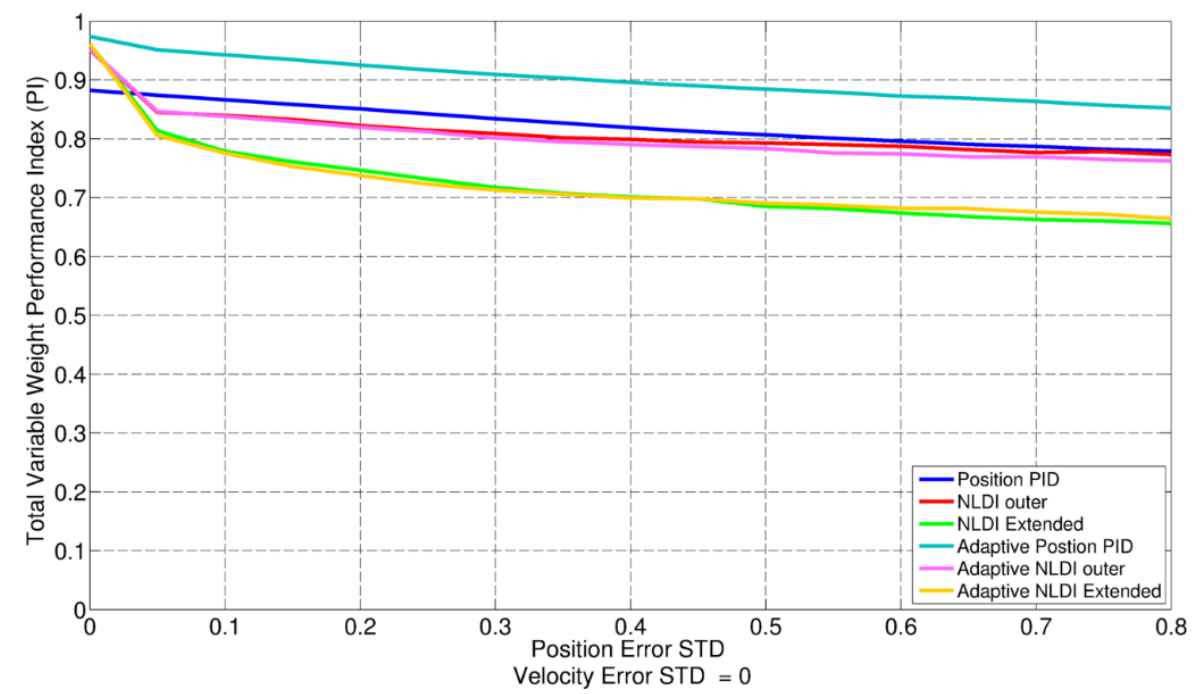

Figure 41: Controllers' PI vs. Position Noise Error STD (Using Variable Weight Algorithm)

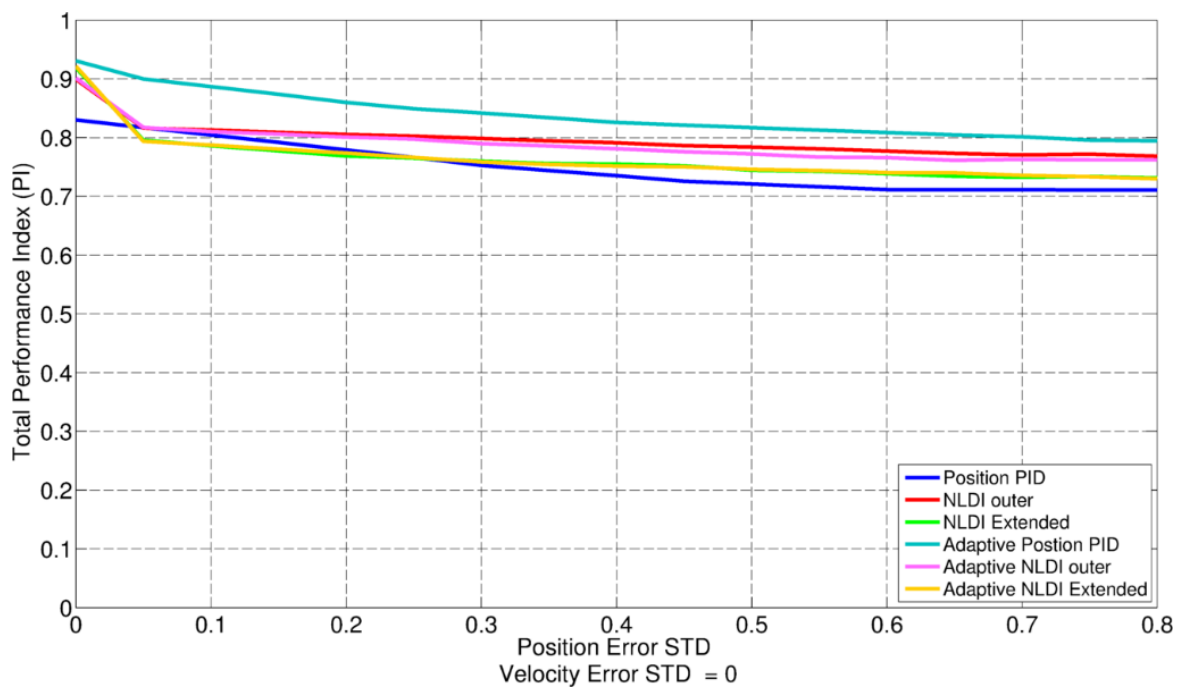

Figure 42: Controllers' PI vs. Position Noise Error STD (Using Fixed Weight Algorithm)

Both algorithms show the degradation in the controllers' performance as the measurement noise increases; however, they rank the controllers' differently. For example, the position PID is ranked the second best controller by the variable weight algorithm on Figure 41, while it is ranked the worst controller by the fixed weight algorithm on Figure 42. A closer look at the controllers' overall TTI (Figure 43) and CAI (Figure 44) helps to explain this difference. The position PID controller's TTI was the lowest (i.e., highest trajectory error), while its CAI was the highest (i.e., lowest controller activity). It is difficult to see the way the variable weight algorithm calculated 
the finial PI from these figures; however, it is obvious that the high CAI helped the controller to get a higher rank. On the other hand, the fixed weight performance index algorithm kept the controller's rank last, because it's low TTI.

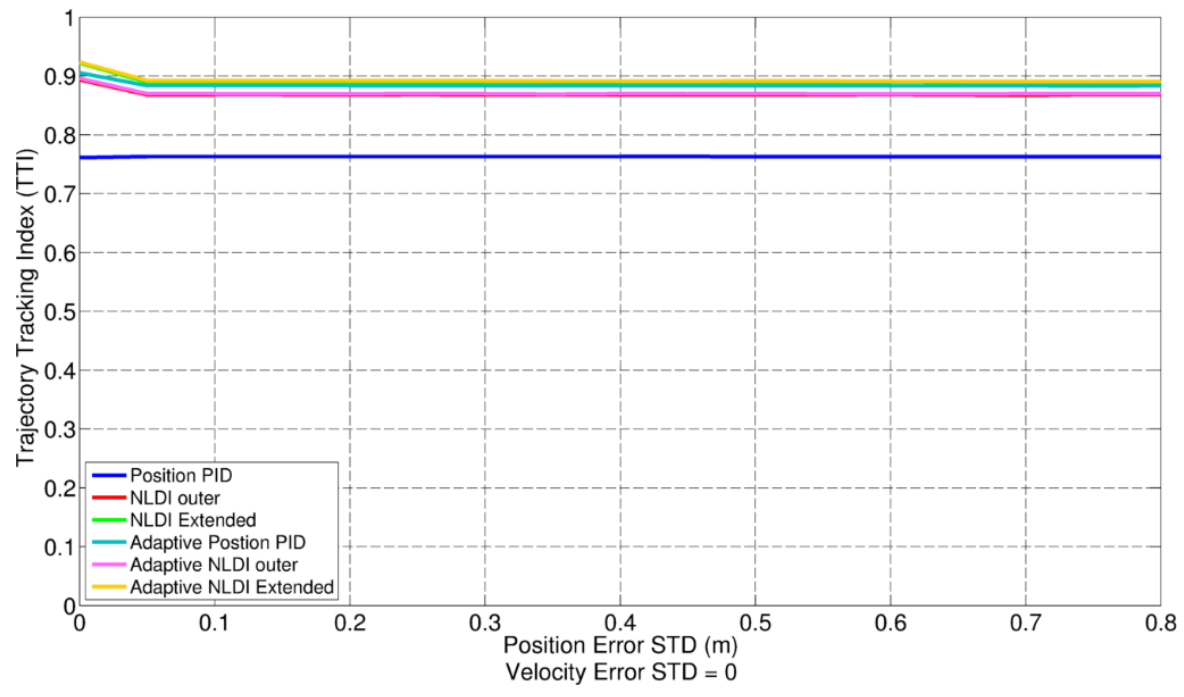

Figure 43: Controllers' TTI vs. Position Noise Error STD

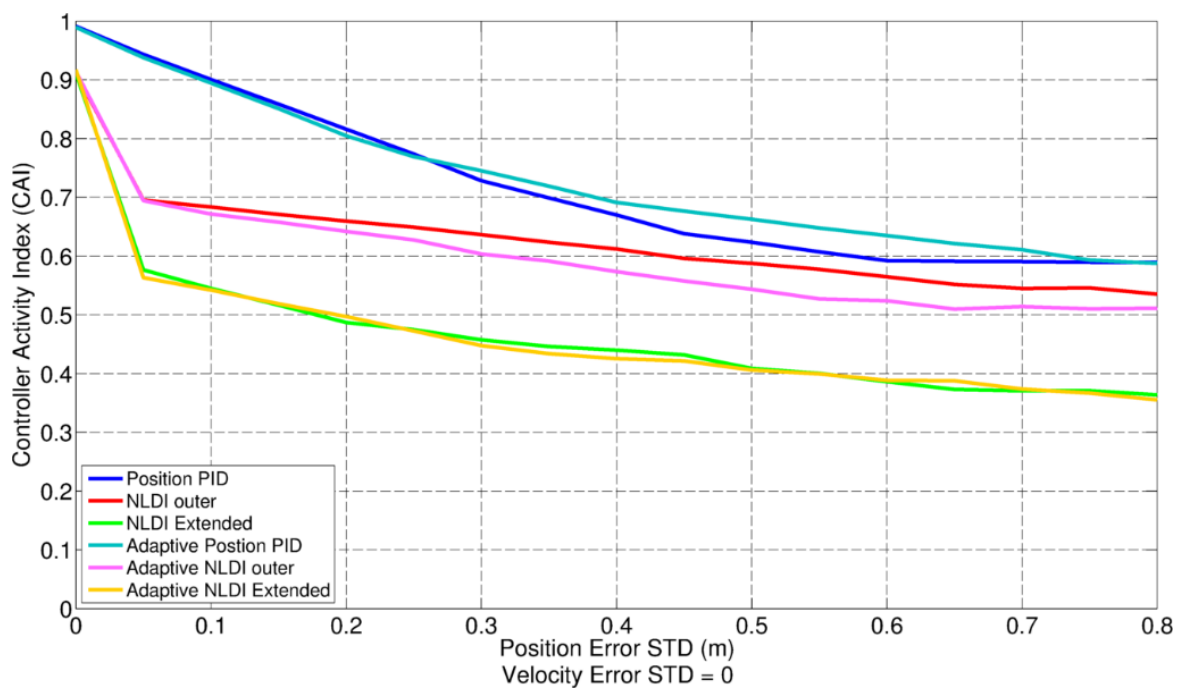

Figure 44: Controllers' CAI vs. Position Noise Error STD

\subsection{Time Delay Sensitivity Analysis}

A time delay sensitivity analysis was performed on the various aircraft trajectory tracking controllers. During this test, the position and velocity measurements from the GPS were delayed in the range of 0 to 1 seconds in increments of 0.04 seconds. First, an overview of the test results 
is provided in Figure 45 in terms of the total PI followed by a separation of the performance components, TTI and CAI, provided in Figure 46 and Figure 47 respectively.

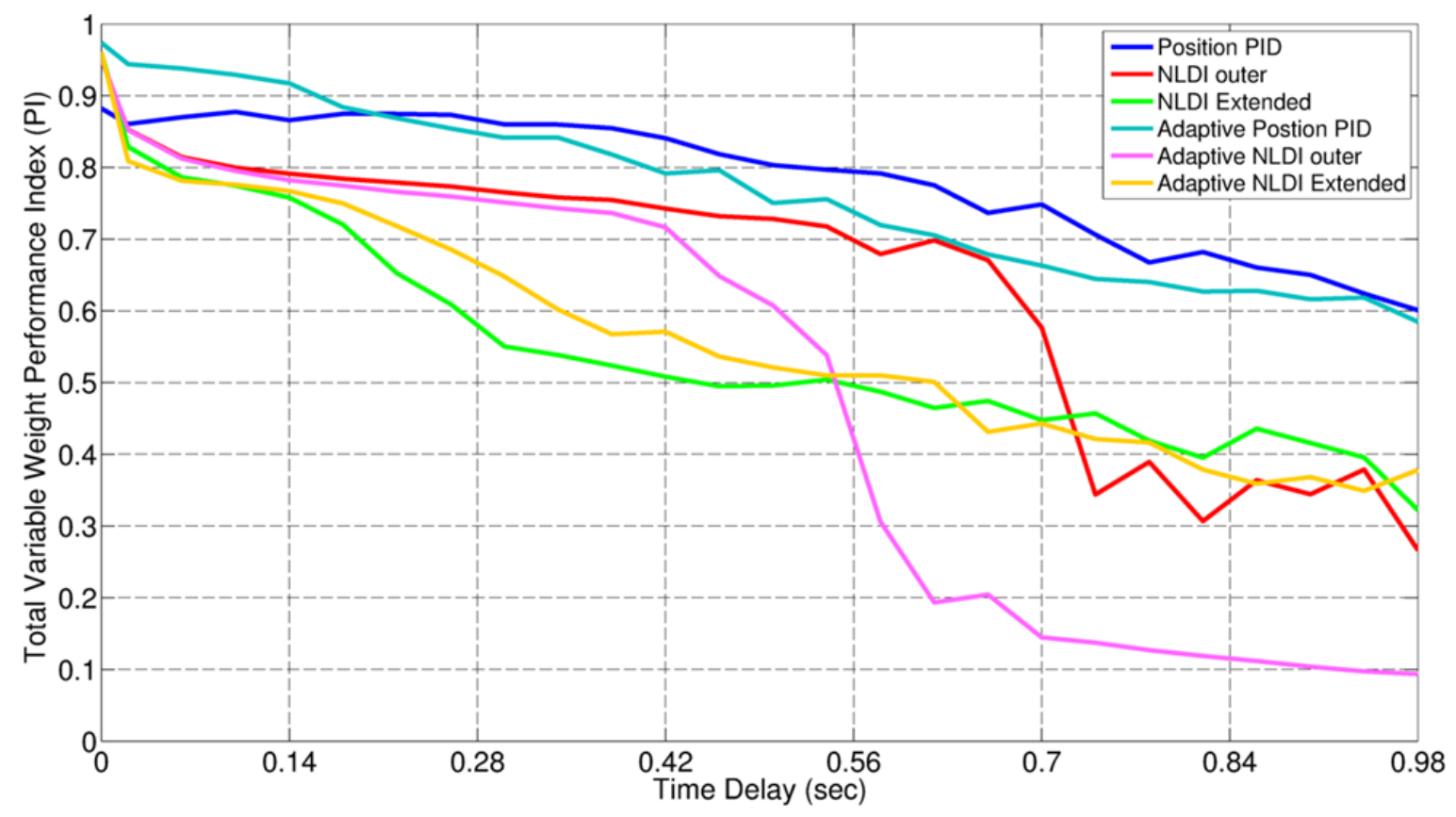

Figure 45: Controllers Performance Index vs. Time Delay

As demonstrated by the simulation results, the performance of the trajectory tracking controllers generally declines as more severe delays are introduced, as expected. Additionally, the percent decrease of controller performance is provided in Table 12. The comparative ranks of the controllers at various times are provided in Table 11 below. The percentage decrease is calculated using the performance index at zero time delay and 0.98 seconds time delay.

\begin{tabular}{|c|c|c|}
\hline Time delay & Best controller & Worst controller \\
\hline $0-0.20$ & Adaptive Position PID & NLDI Extended \\
\hline $0.20-0.52$ & Position PID & NLDI Extended \\
\hline $0.52-0.98$ & Position PID & Adaptive outer \\
\hline
\end{tabular}

Table 11: Best and Worst Controllers in the Presence of Time Delays 


\begin{tabular}{|c|c|}
\hline Controller Name & Percentage decrease in Total Performance \\
\hline Position PID & $19 \%$ \\
\hline NLDI Outer & $70 \%$ \\
\hline NLDI Extended & $66 \%$ \\
\hline Adaptive Position PID & $30 \%$ \\
\hline Adaptive NLDI Outer & $89 \%$ \\
\hline Adaptive NLDI Extended & $63 \%$ \\
\hline
\end{tabular}

Table 12: Percentage Decrease in PI Due to Time Delay

In terms of the total PI, it can be noticed that the fixed and adaptive PID control laws exhibit the highest robustness with respect to time delays. However, it should be noted that this is primarily due to a lack of control activity and that in terms of tracking errors, the PID controllers outperformed at low levels of time delays (up to approximately $0.2 \mathrm{sec}$ ). The more complex controllers experience a significant increase of control activity for delays above 0.2 sec with a tracking accuracy which was lower than expected. The adaptive mechanism applied to the fixed parameter baseline controllers appears to have a positive effect only over limited ranges of time delays.

The position PID controller, with a maximum drop of performance of $19 \%$, was the least affected by delays in GPS location and velocity measurements. The adaptive outer-loop NLDI was the least robust to GPS delays, with a maximum performance decrease of $89 \%$. Both the outer-loop NLDI and its adaptive counterpart experienced a sharp decrease of performance when the delay reached a magnitude of around 0.56 seconds, whereas the other controllers exhibited a more gradual decline in performance. The adaptive controllers exhibited little to no improvement over their fixed-parameter counterparts in response to GPS time delays.

For more insight into the effect of the time delay on the controllers' trajectory tracking performance, Figure 46 and Figure 47 illustrate the performance components, trajectory tracking index (TTI) and the controller activity index (CAI) respectively. 


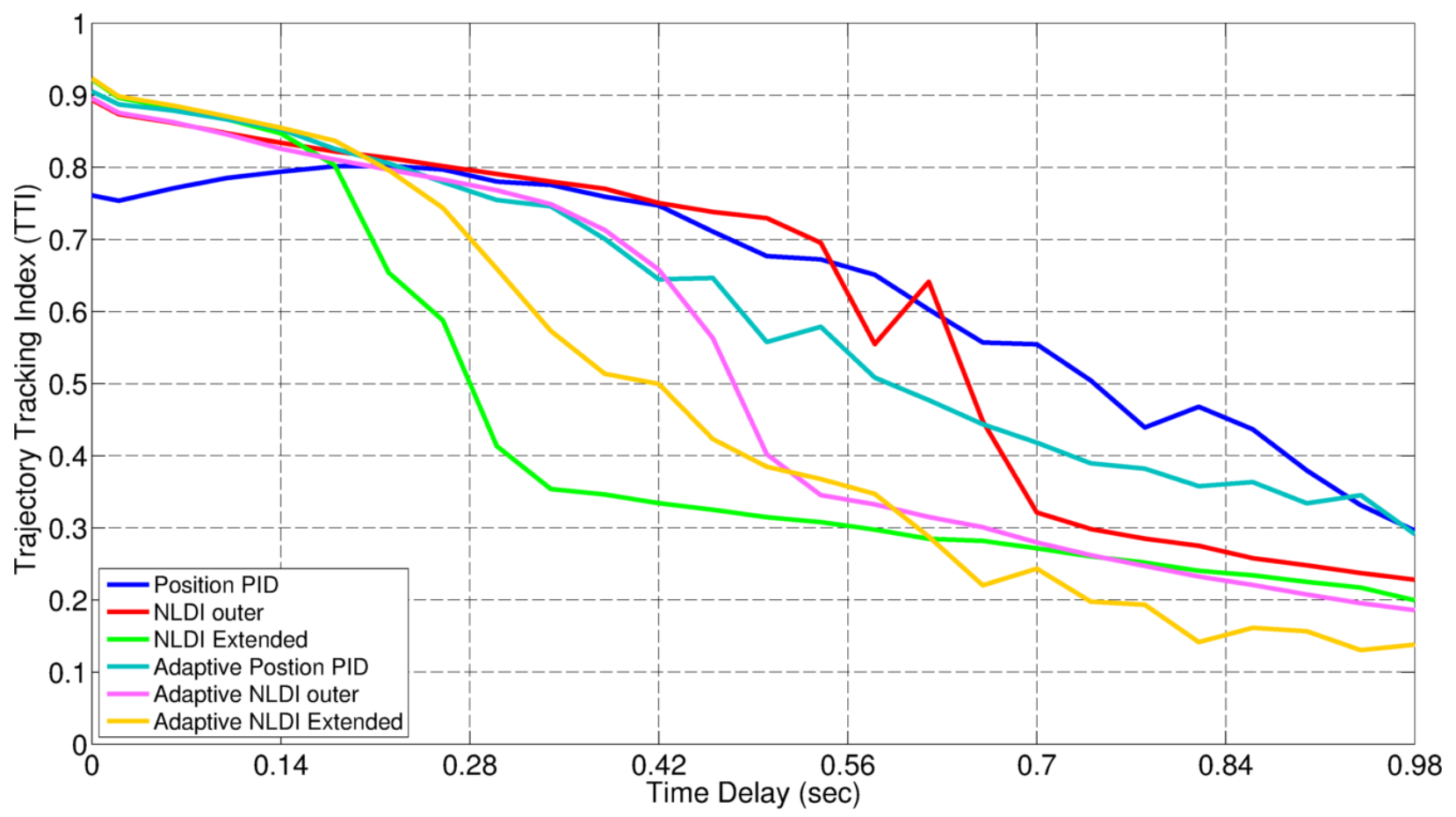

Figure 46: Controllers' TTI Index vs. Time Delay

The variation of the TTI reveals the position PID robustness to the time delay in the measured position and velocity values, while the NLDI controller has a sharp decrease in the TTI after about 0.20 seconds delay. The NLDI extended drops about $63 \%$ of its TTI at 0.42 sec time delay while the position PID controller keeps its TTI at the same level.

The variation of the CAI, shows little impact on the position PID and the adaptive position PID controllers' CAl; both controllers preserve low controller activity throughout the time delay analysis. Significant impact can be seen on the extended NLDI and adaptive extended NLDI controllers' CAI, both controllers drop $45 \%$ of their CAI at 0.14 sec time delay; however, they keep the 0.14 sec CAI value for the rest of the analysis. This effect is less severe on the NLDI outer and the adaptive NLDI outer; however, a sharp drop in the adaptive NLDI controller's CAI can be seen for time delays longer than $0.56 \mathrm{sec}$. Generally, the sudden drop in the NLDI controllers is due to the saturation in the throttle command. The NLDI controllers use a non-linear approach to calculate the throttle command based on required acceleration to compensate for position error. The approach works well for errors within a certain range. Certain time delay values (or any other source error) produce large errors that exceed this range and the throttle command saturates with damaging effects on the control performance in the aircraft plane of symmetry and 
departure from the commanded trajectory. The throttle control model must be revised to avoid this problem.

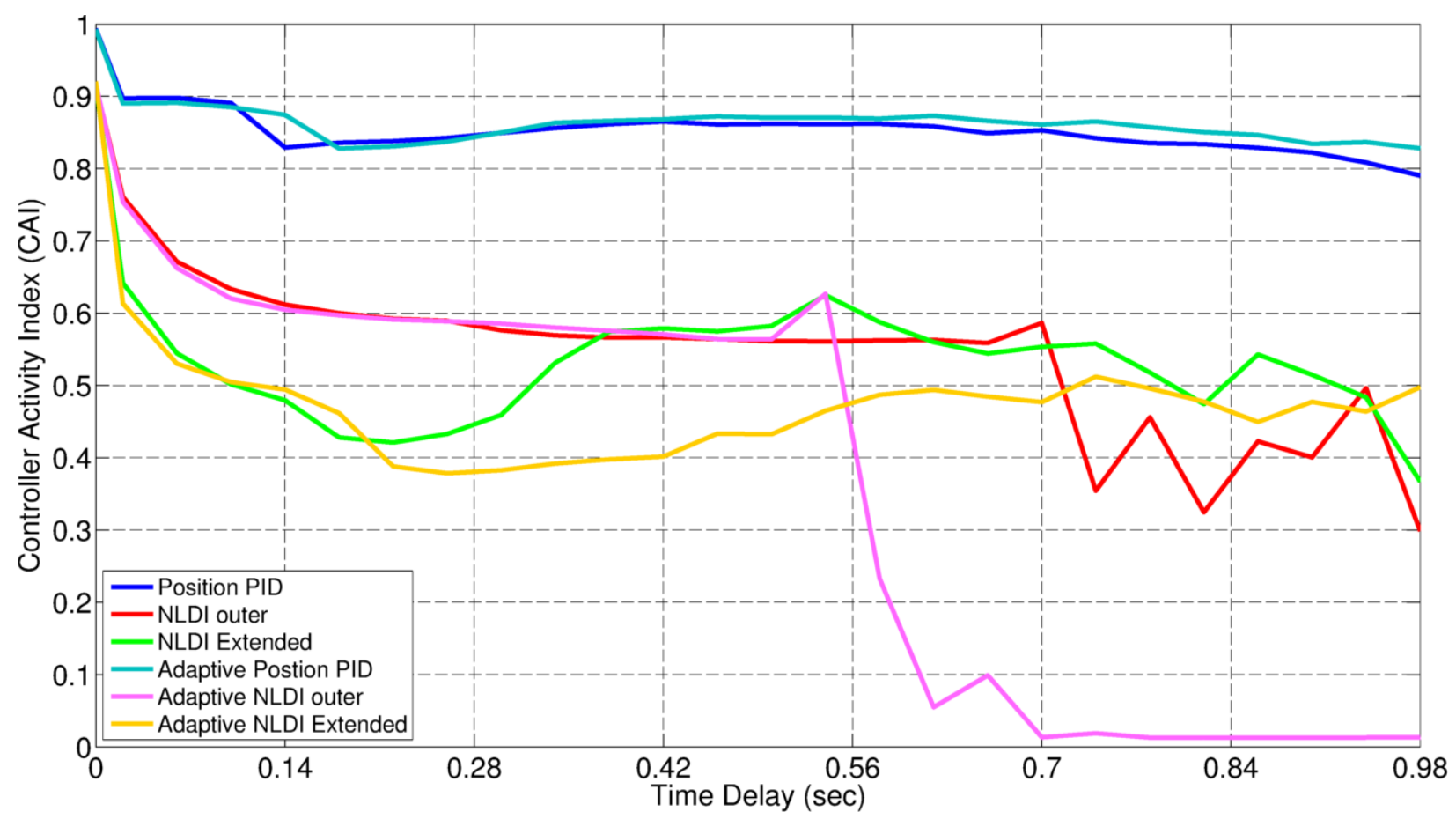

Figure 47: Controllers' CAI Index vs. Time Delay

From the tests performed, it can be concluded that "good" performance can be maintained by the controllers investigated up to time delays between 0.2 and $0.72 \mathrm{sec}$. The control of the vehicle will be lost if the time delays exceed thresholds in the range $0.56-0.94 \mathrm{sec}$, depending on the controller.

An example showing the commanded and the actual trajectory is presented in Figure 48 for the Position PID controller, and in Figure 49 for the adaptive NLDI outer controller both with a time delay of 0.54 seconds. Figure 50 and Figure 51 represent the corresponding controllers' surface deflection for each case. 


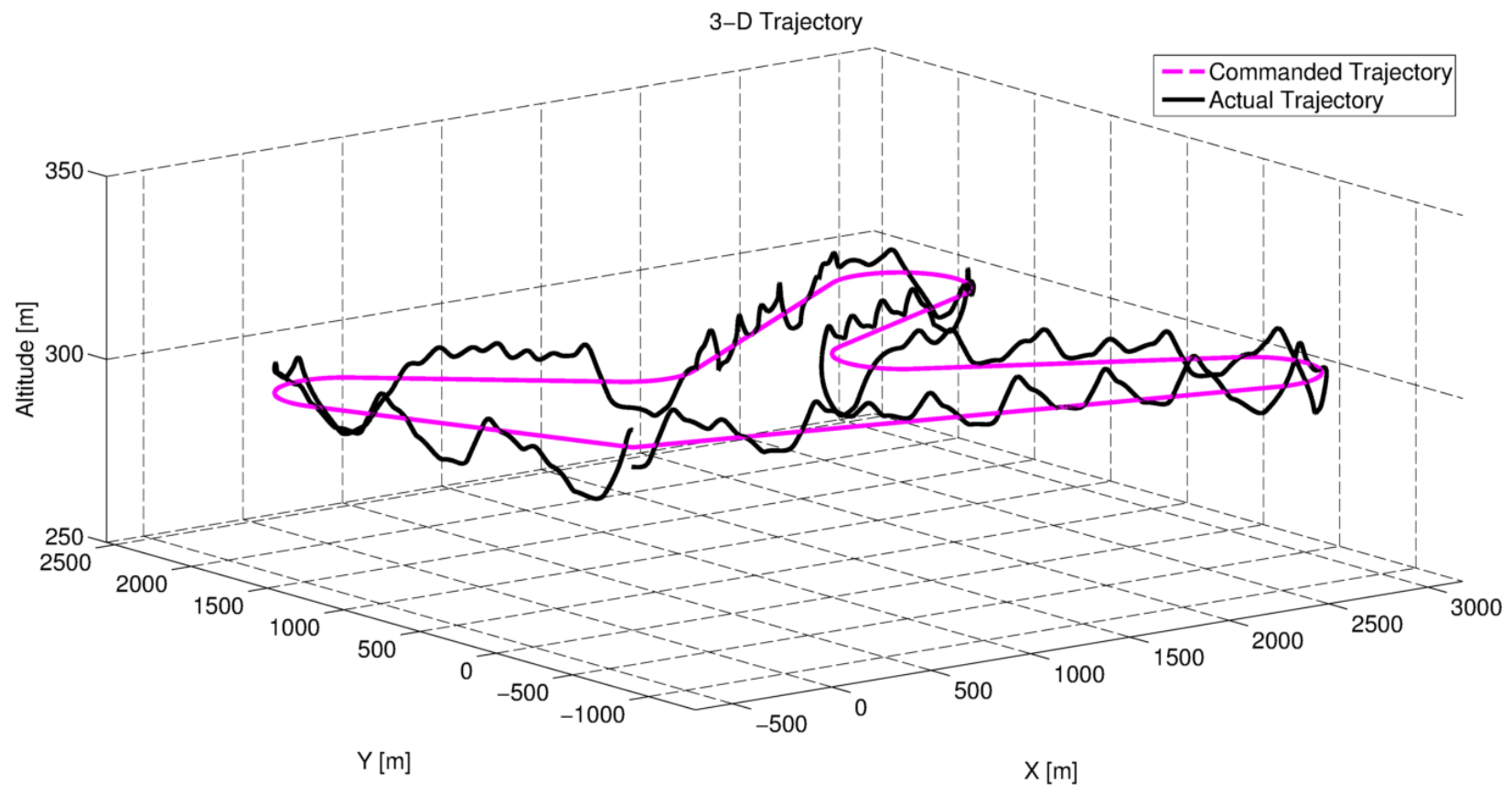

Figure 48: Commanded and Actual Trajectory for Position PID at $0.54 \mathrm{sec}$ Time Delay

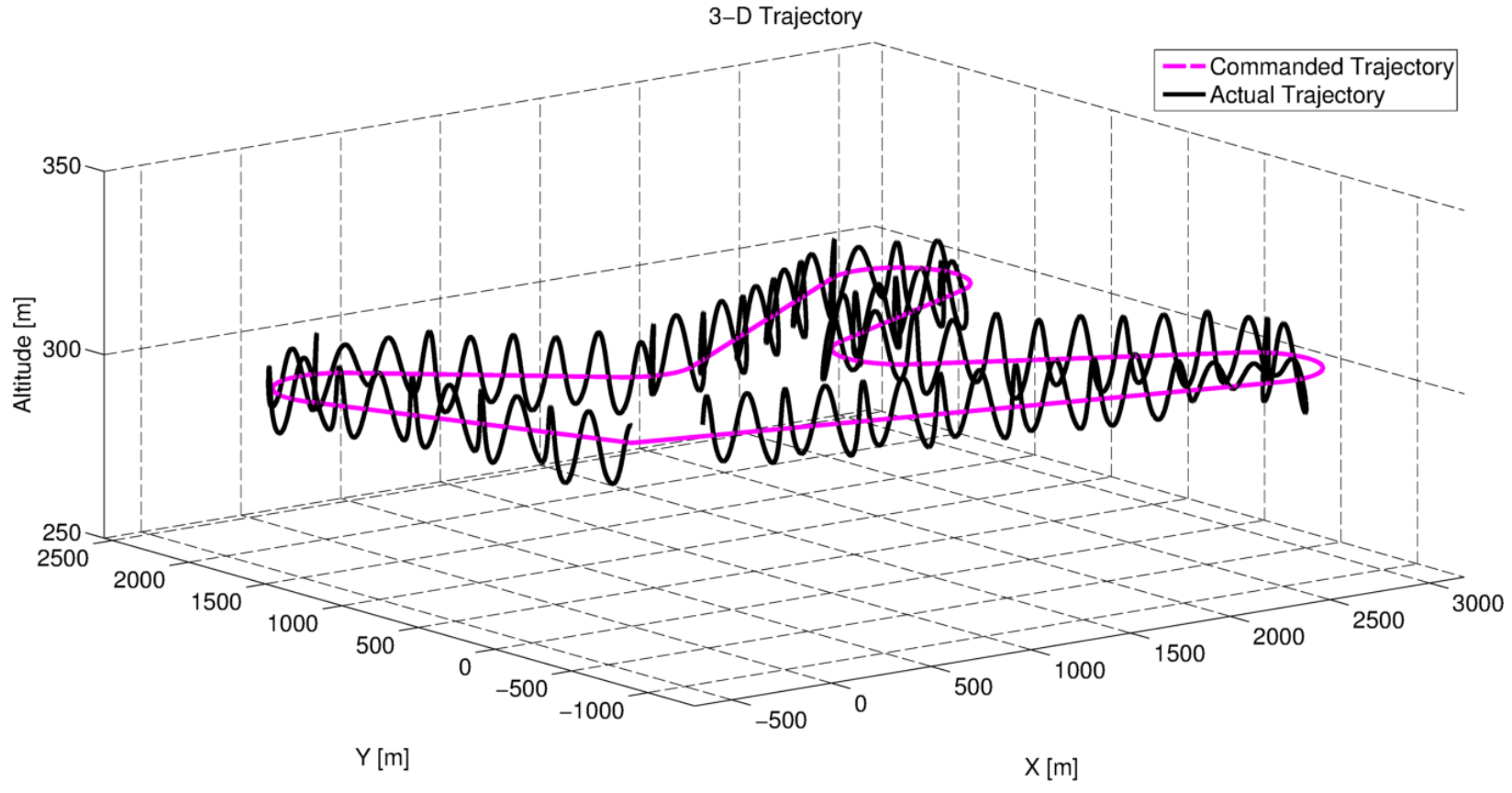

Figure 49: Commanded and Actual Trajectory for Adaptive Outer at $0.54 \mathrm{sec}$ Time Delay 

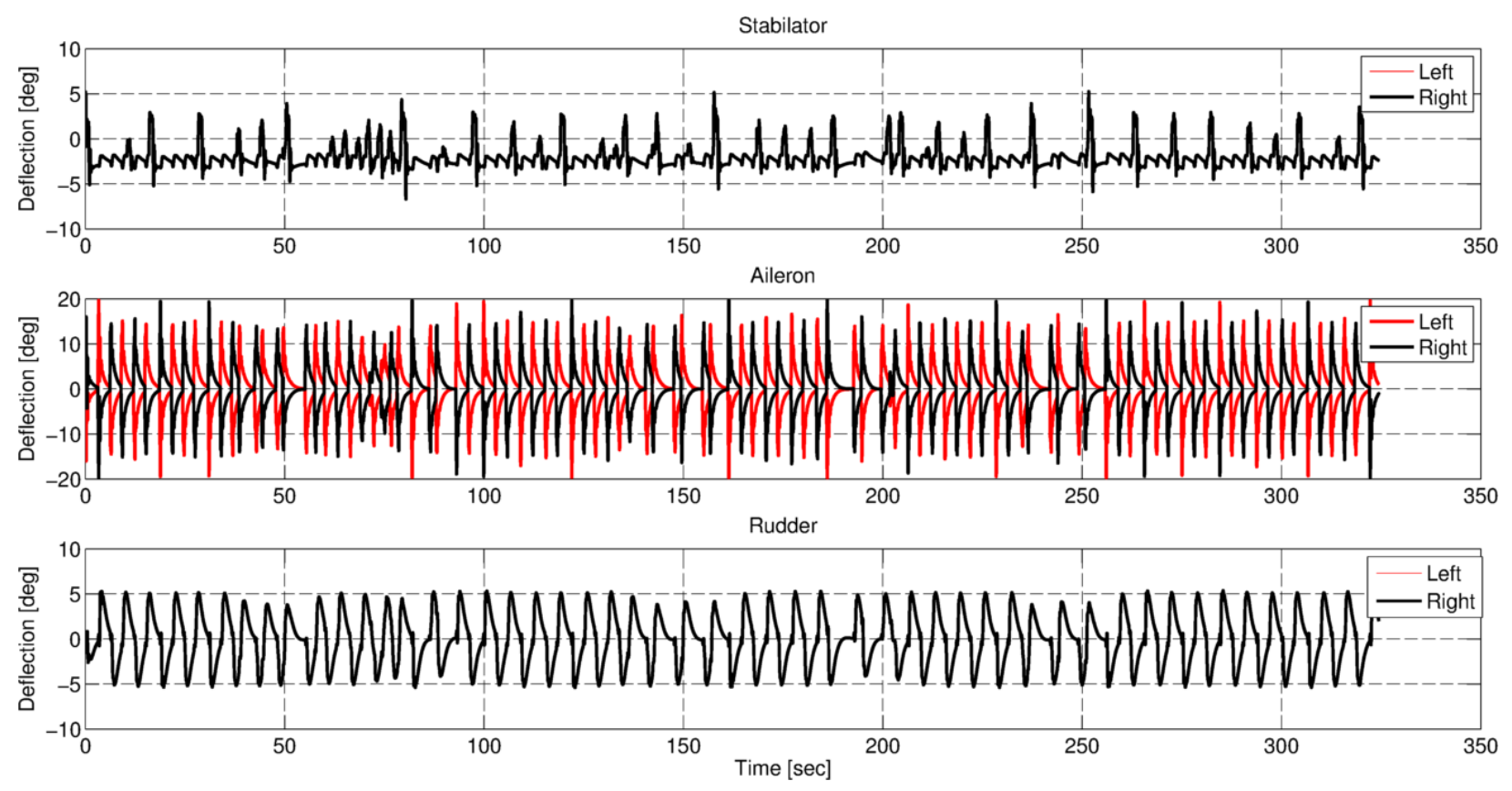

Figure 50: Controller Surfaces Deflections for Position PID at $0.54 \mathrm{sec}$ Time Delay
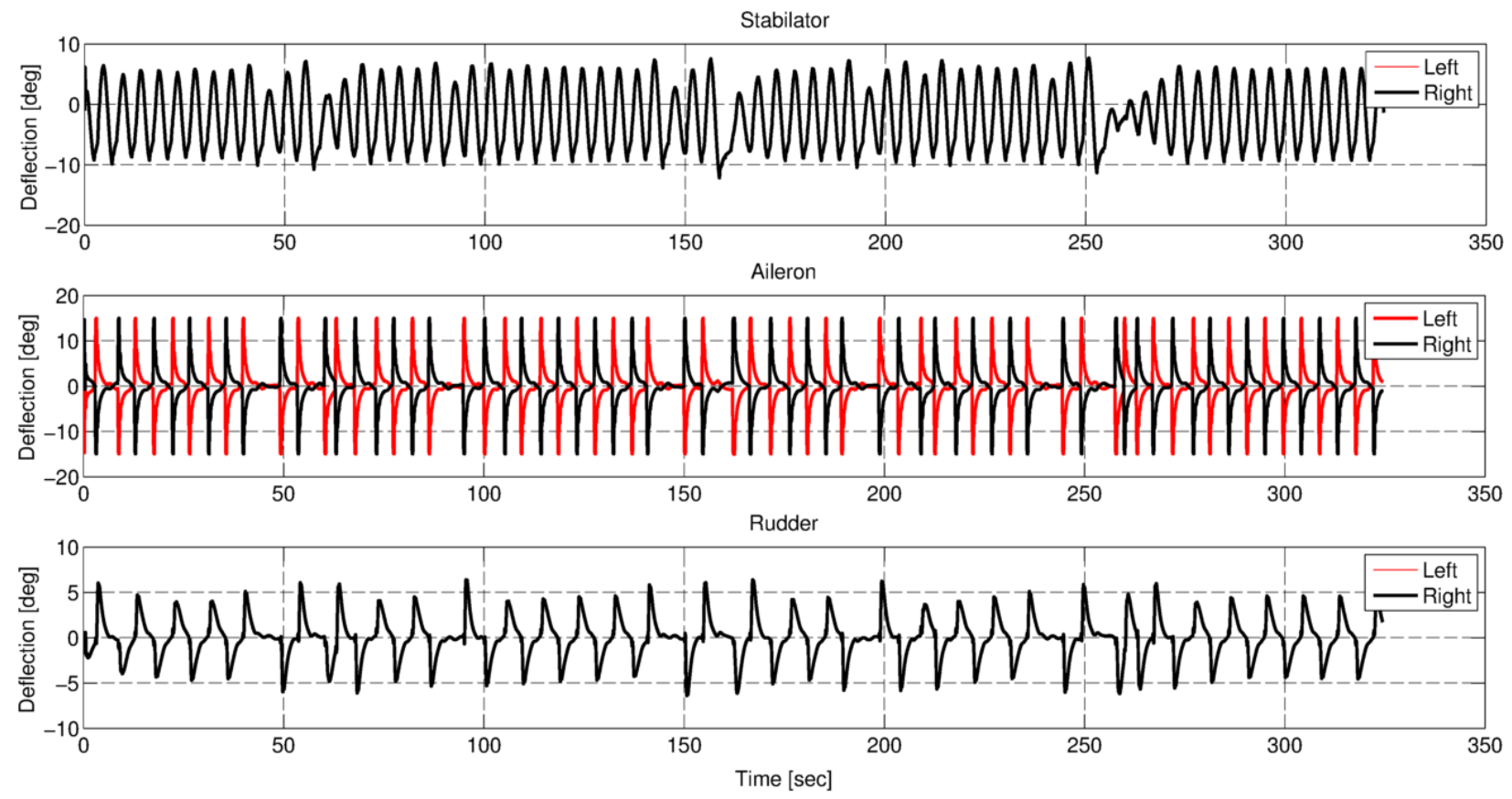

Figure 51: Controller Surfaces Deflections for Adaptive Outer at $0.54 \mathrm{sec}$ Time Delay

\subsection{Update Interval Sensitivity Analysis}

The update Interval sensitivity analysis tested the controllers' trajectory tracking performance against the position and velocity update Intervals. In the first analysis, the position and velocity have equal update Intervals in the range of 0.02 and 1 seconds. The actual receiver update varies 
from 0.02 to 1 seconds. An overview of the test results is provided in Figure 52 in terms of the total PI followed by a separation into performance index components, TTI and CAl, in Figure 53 and Figure 54, respectively. Additionally, the comparative ranking of the controllers for various update ranges is provided in Table 13 below.

\begin{tabular}{|c|c|c|}
\hline Update Interval & Best Controller & Worst Controller \\
\hline $0.02-0.14$ & Adaptive Position PID & NLDI Extended \\
\hline $0.14-0.98$ & Position PID & NLDI Extended \\
\hline
\end{tabular}

Table 13: Best and Worst Controllers for Different Update Interval Values

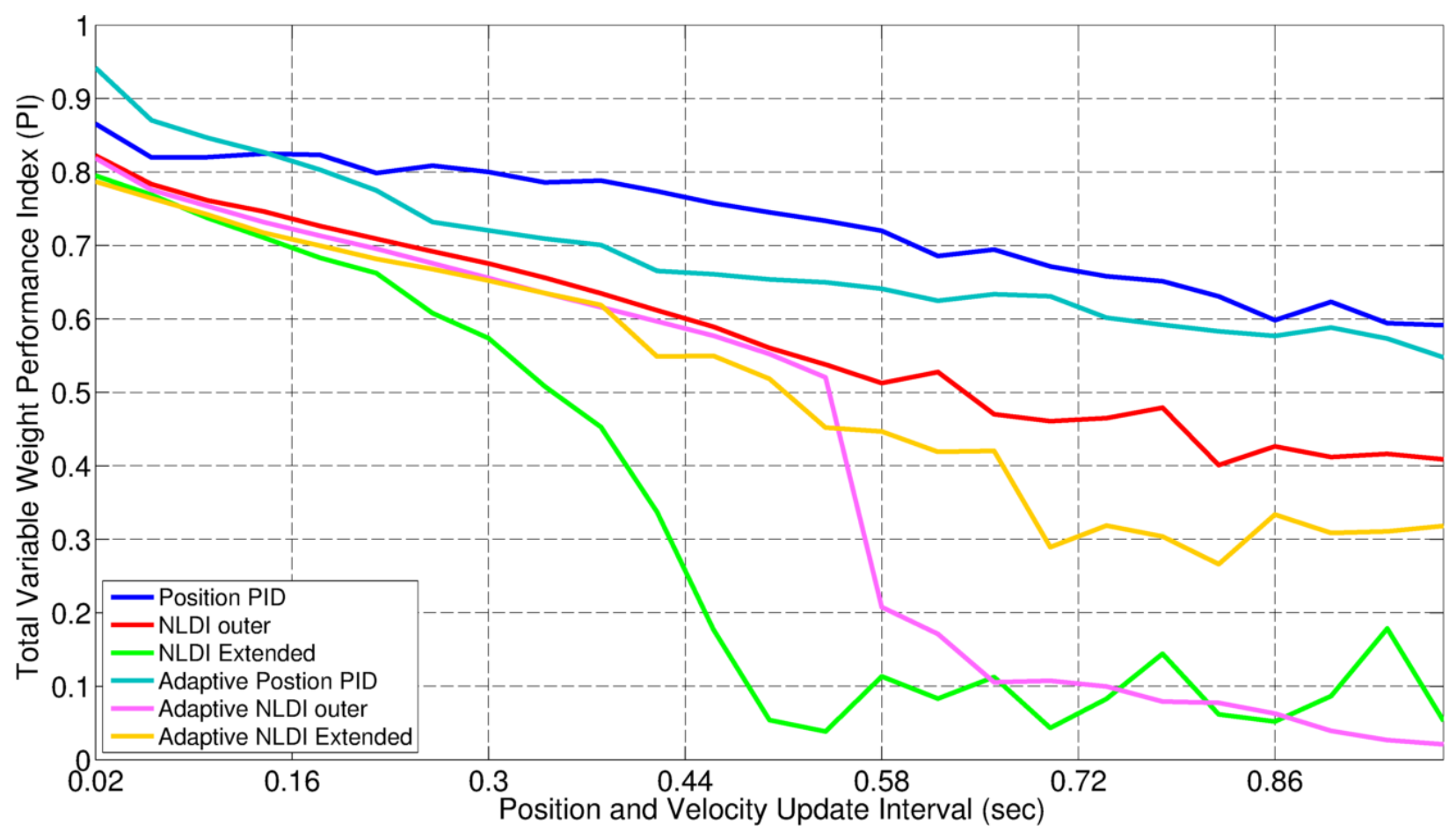

Figure 52: Controllers Performance Index vs. Position and Velocity Update Interval

Both the NLDI extended and the adaptive outer controllers exhibit a sharp decrease of their performances when the position and velocity update intervals increase to 0.38 and 0.54 seconds, respectively, while the rest of the controllers performances alter more gradually as larger update interval were introduced. Some controllers show the same behavior they exhibited in the presence of the time delay (e.g. position PID and adaptive position PID); however, some other controllers exhibit entirely different behavior. Table 14 summarizes the percentage decrease of controller performance in the update interval category along with the time delay results. 


\begin{tabular}{|c|c|c|}
\hline Controller Name & $\begin{array}{c}\text { Percentage Decrease in Total } \\
\text { Performance Due to update } \\
\text { Interval }\end{array}$ & $\begin{array}{c}\text { Percentage Decrease in Total } \\
\text { Performance Due to Time Delay }\end{array}$ \\
\hline Position PID & $17 \%$ & $19 \%$ \\
\hline NLDI Outer & $50 \%$ & $70 \%$ \\
\hline NLDI Extended & $87 \%$ & $66 \%$ \\
\hline Adaptive Position PID & $30 \%$ & $30 \%$ \\
\hline Adaptive NLDI Outer & $87 \%$ & $89 \%$ \\
\hline Adaptive NLDI Extended & $56 \%$ & $63 \%$ \\
\hline
\end{tabular}

Table 14: Update Interval and Time Delay Results Comparison

The variation of TTI shows that the position PID controller exhibits robustness with respect to the update interval as it kept or improved the TTI for update Intervals up to 0.5 seconds, while the NLDI controller totally lost the TTI index to zero for update intervals beyond 0.42 seconds. In general, the controllers' TTI exhibited more sever degradation in the update interval analysis than in the time delay analysis.

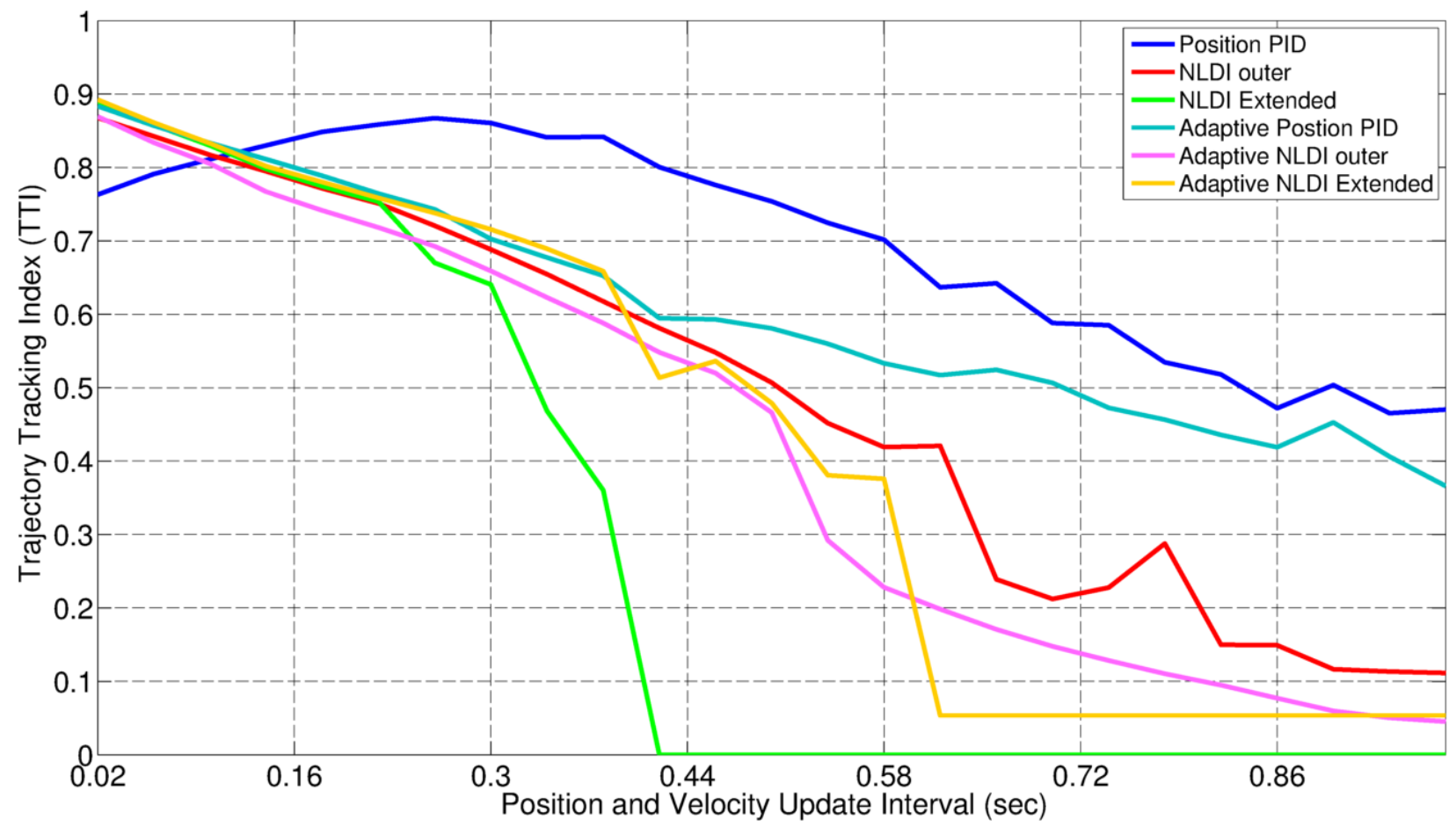

Figure 53: Controllers' TTI Index vs. Position and Velocity Update Interval 
The update interval variation not only affects the TTI of the NLDI extended controller, it also drops the NLDI extended controller's CAI to less than 0.1 for an update interval of 0.45 seconds. The adaptive outer controller also has a sharp drop in its CAI at 0.60 seconds update interval. The position PID and the adaptive position PID controllers drop $0.30 \%$ of their CAI at $0.22 \mathrm{sec}$; however, they maintain the CAI at the same level for the rest of the range.

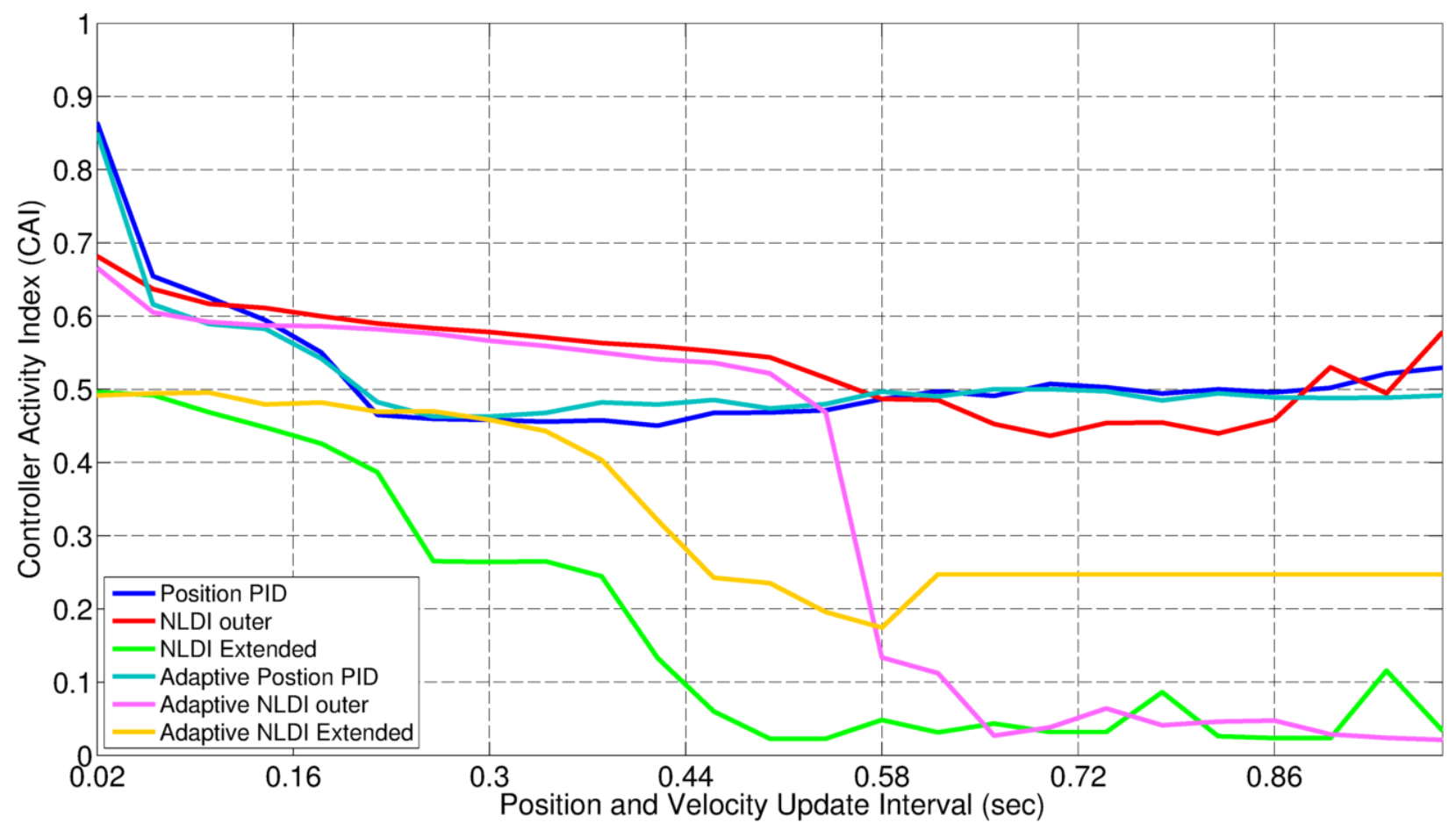

Figure 54: Controllers' CAI Index vs. Position and Velocity Update Interval

Figure 55, and Figure 56 illustrate the actual and the commanded trajectories at position and velocity update interval of 0.44 seconds for the position PID and the NLDI extended controllers respectively. 


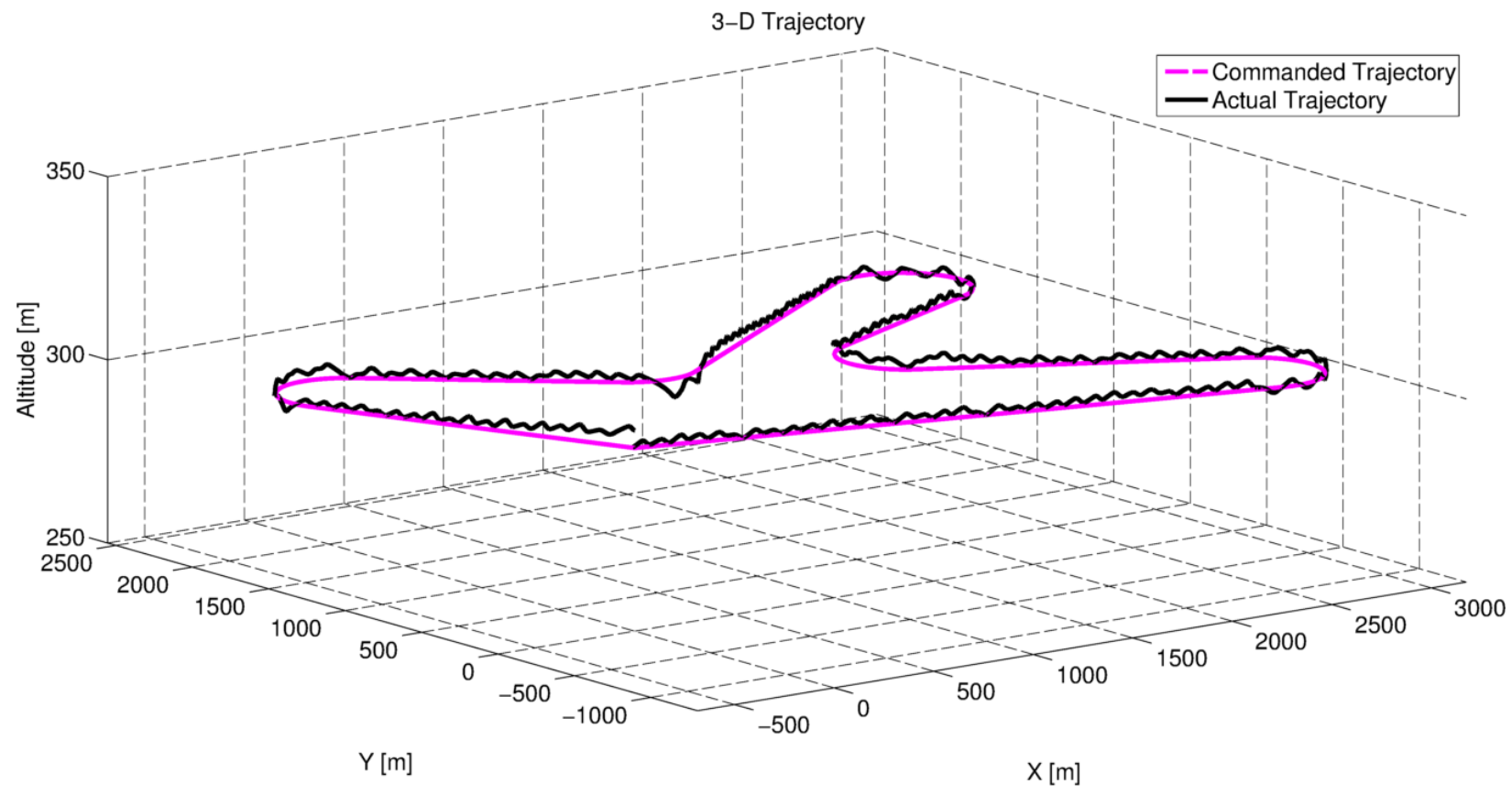

Figure 55: Commanded and Actual Trajectories for Position PID at $0.44 \mathrm{sec}$ Position and Velocity Update Interval

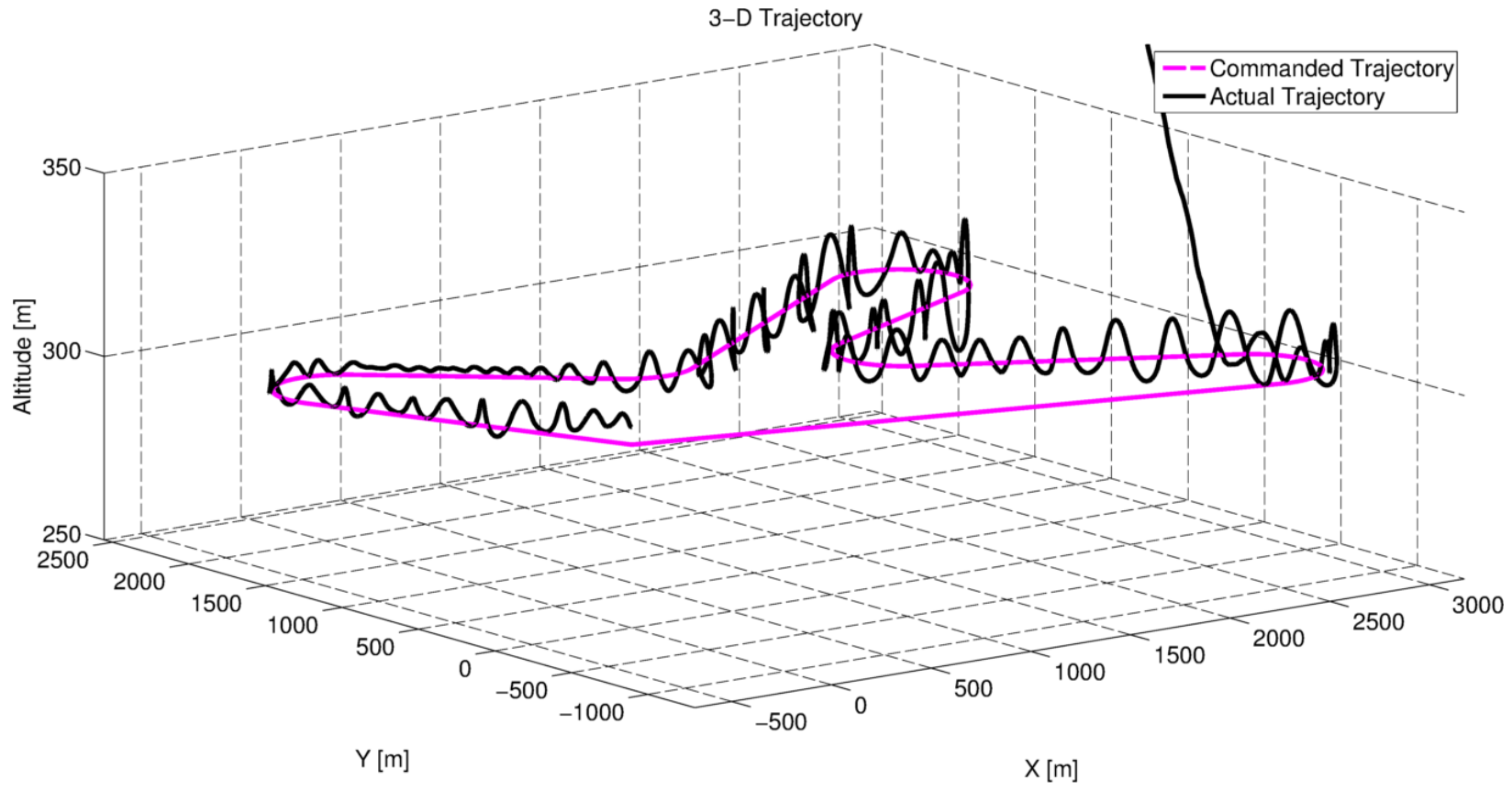

Figure 56: Commanded and Actual Trajectories for NLDI extended at 0.44 sec Position and Velocity Update Interval

Figure 61and Figure 62 illustrate the controllers' surface deflection at position and velocity update interval of 0.44 seconds for the position PID and the NLDI extended controllers respectively. 
Stabilator
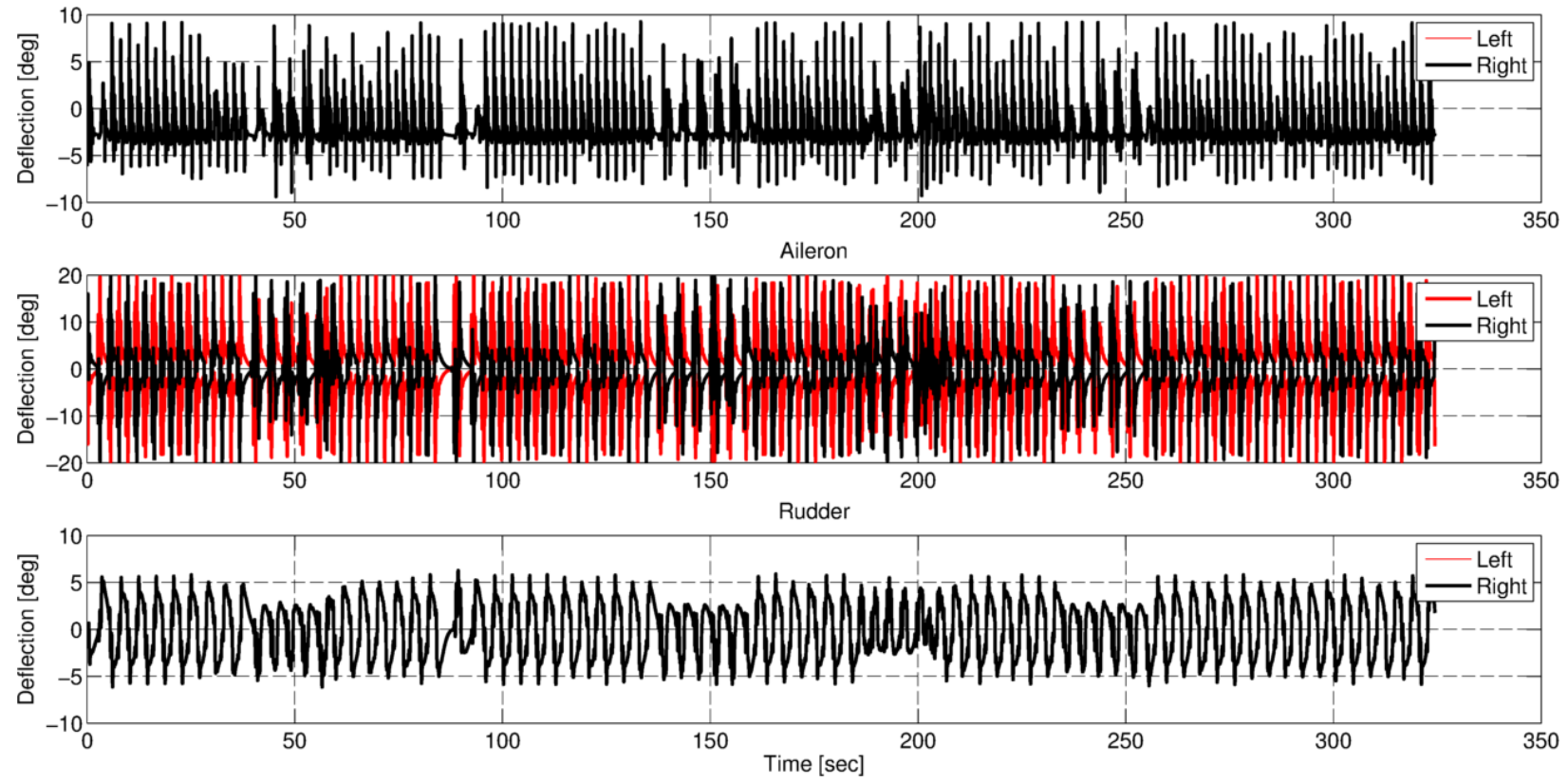

Figure 57: Controller Surfaces Deflections Position PID at $0.44 \mathrm{sec}$ Position and Velocity Update Interval
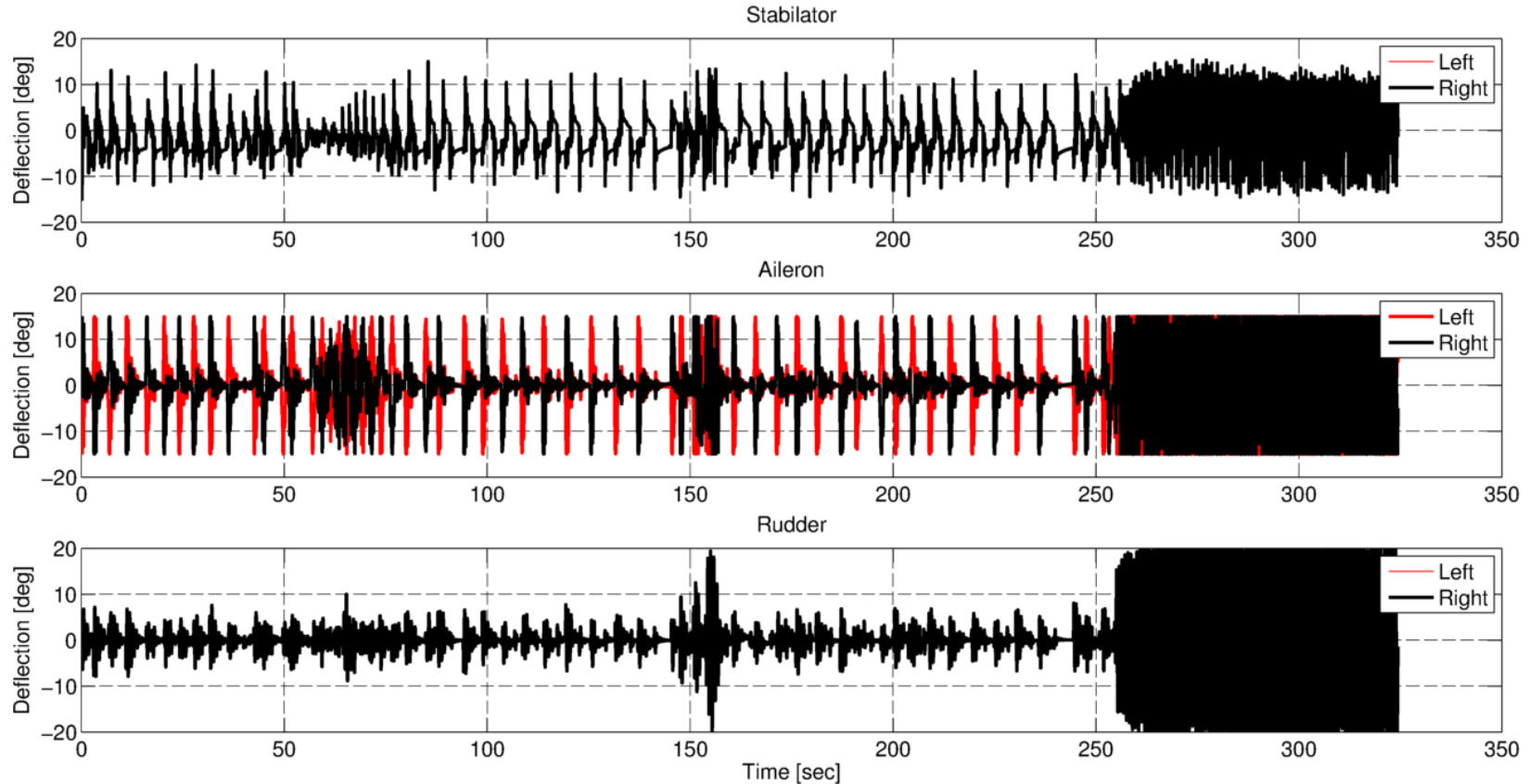

Figure 58: Controller Surfaces Deflections NLDI Extended at $0.44 \mathrm{sec}$ Position and Velocity Update Interval

The second part of the update interval sensitivity analysis, addresses the controllers' trajectory tracking performance with respect to the velocity update intervals. The motivation for this analysis is that some of the commercial receivers have a low update interval for the position and a larger one for the velocity, because their velocity calculation algorithm requires a longer time. During this analysis, the velocity measurements update interval from the GPS were varied in the 
range of 0 to 1 seconds with an increment of 0.08 seconds. The position update interval was 0.02 seconds through all this analysis. An overview of the test results is provided in Figure 59 in terms of the total PI followed by a separation of the constituent parts, TTI and CAI, in Figure 60 and Figure 61, respectively.

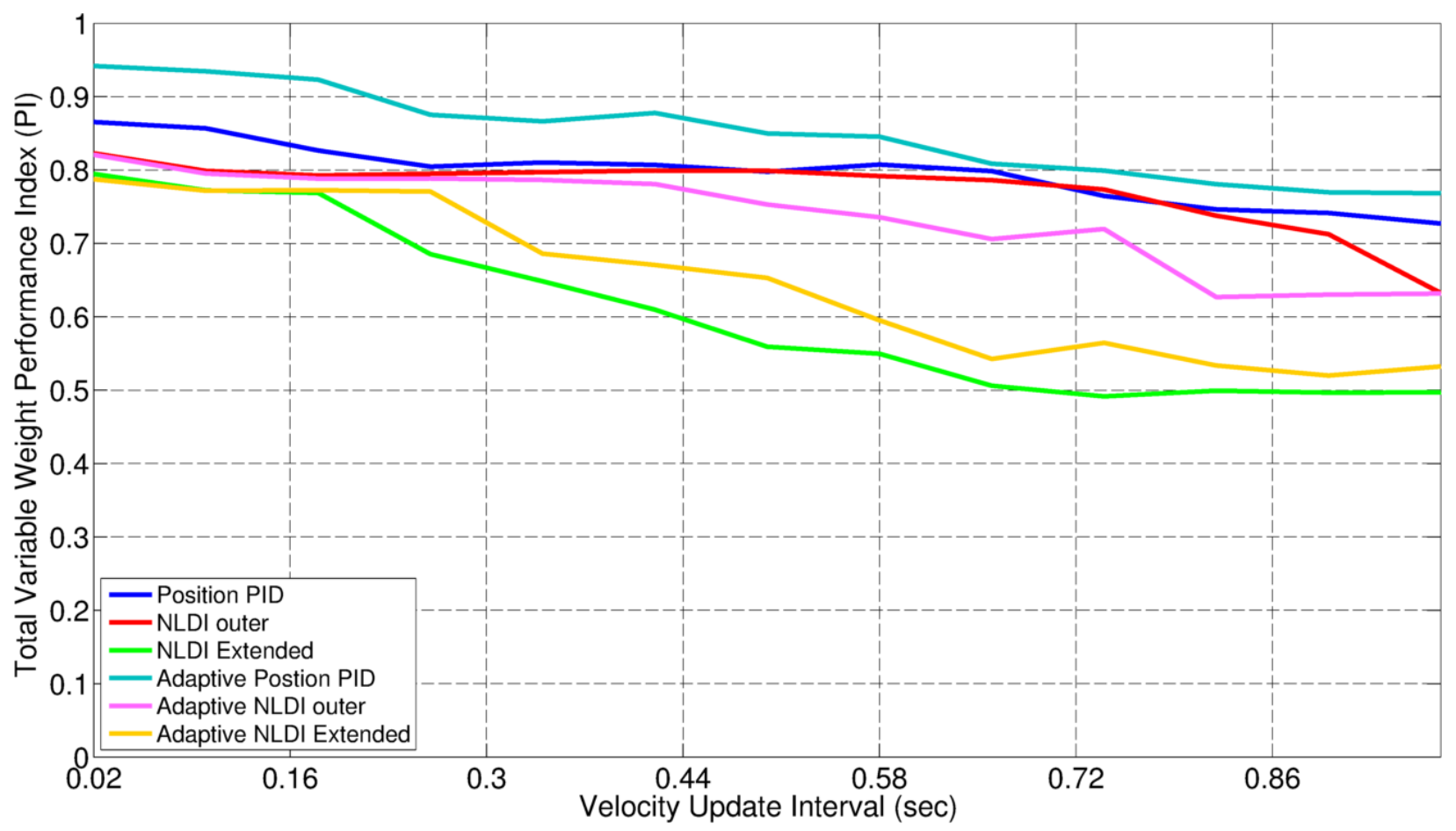

Figure 59: Controllers' Performance Index vs. Velocity Update Interval

The impact of the velocity update Interval on the controller's performances was less severe than the position and velocity update Interval since all the controllers had a PI of 0.5 or better. The position PID controllers were the best performing controllers due to their higher CAI. One explanation for this is that the position PID controller only relies upon the velocity measurements in the trajectory geometry module, whereas the NLDI controllers also uses the velocity measurements in the dynamic inversion process. 


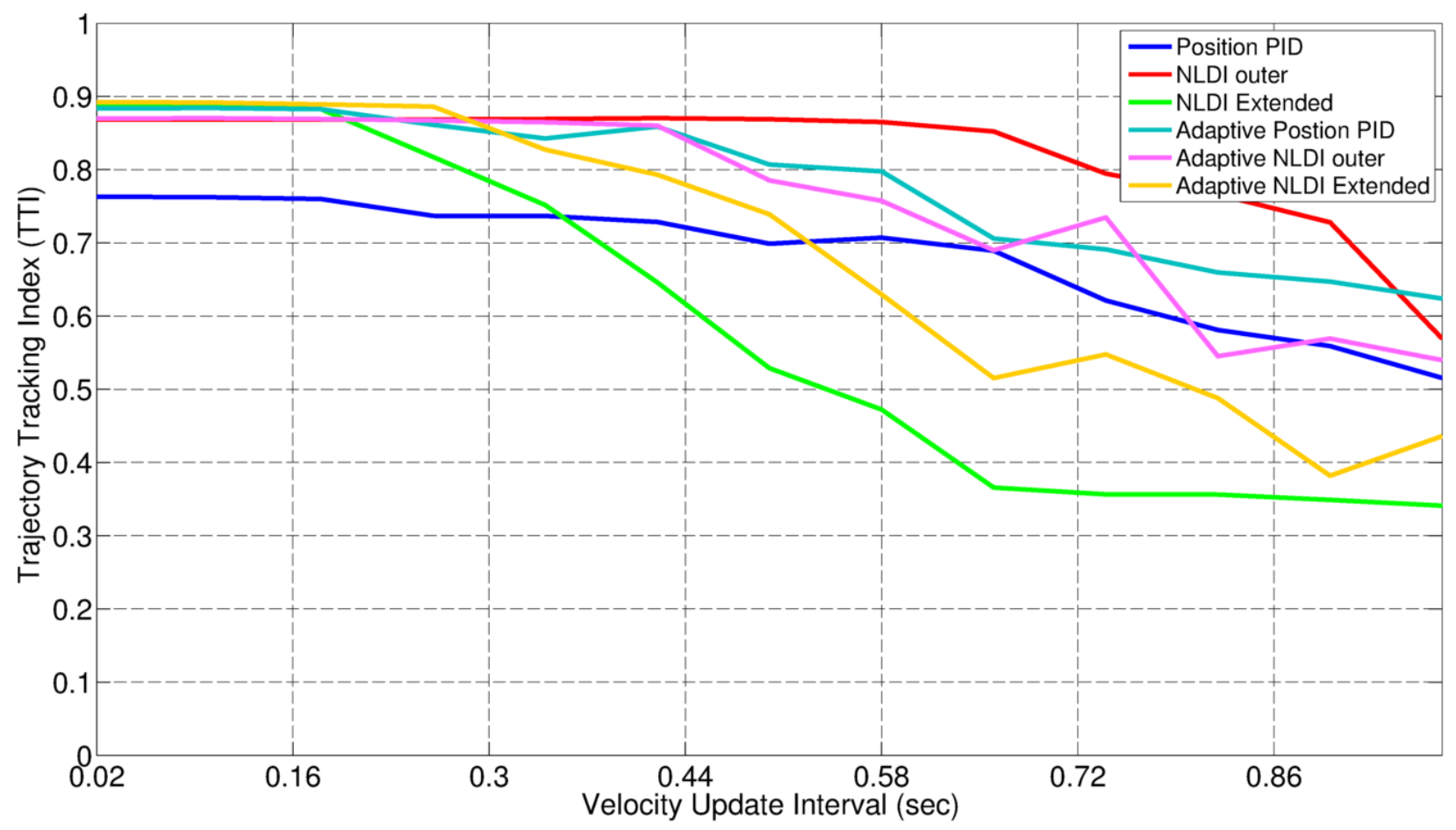

Figure 60: Controllers' TTI Index vs. Velocity Update Interval

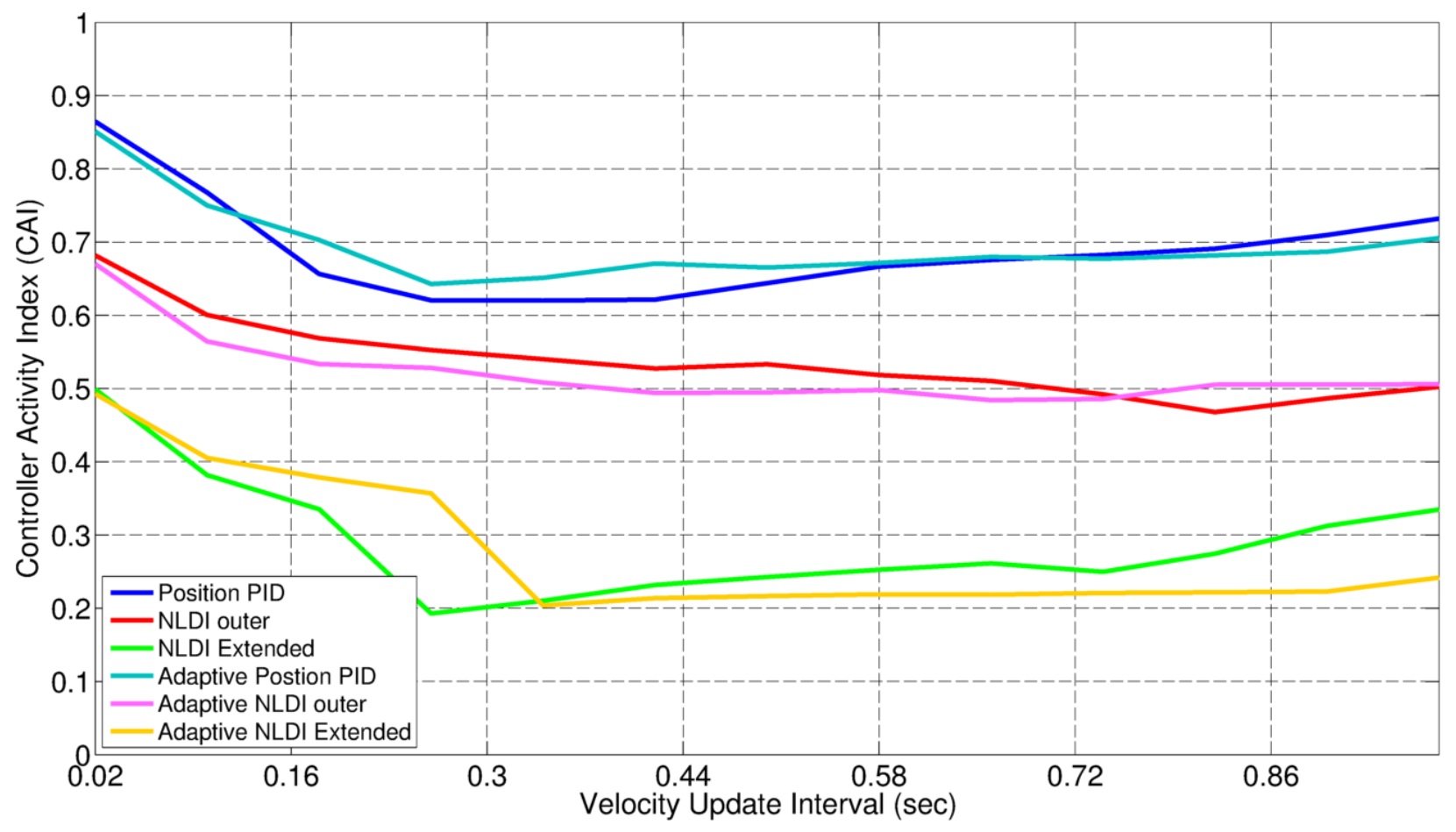

Figure 61: Controllers' CAI Index vs. Velocity Update Interval 
An example of the effect of the velocity update interval on the controllers is shown in Figure 62 and Figure 63, which present the commanded and the actual trajectories for the adaptive position PID and the extended controllers both at $0.98 \mathrm{sec}$ velocity update interval.

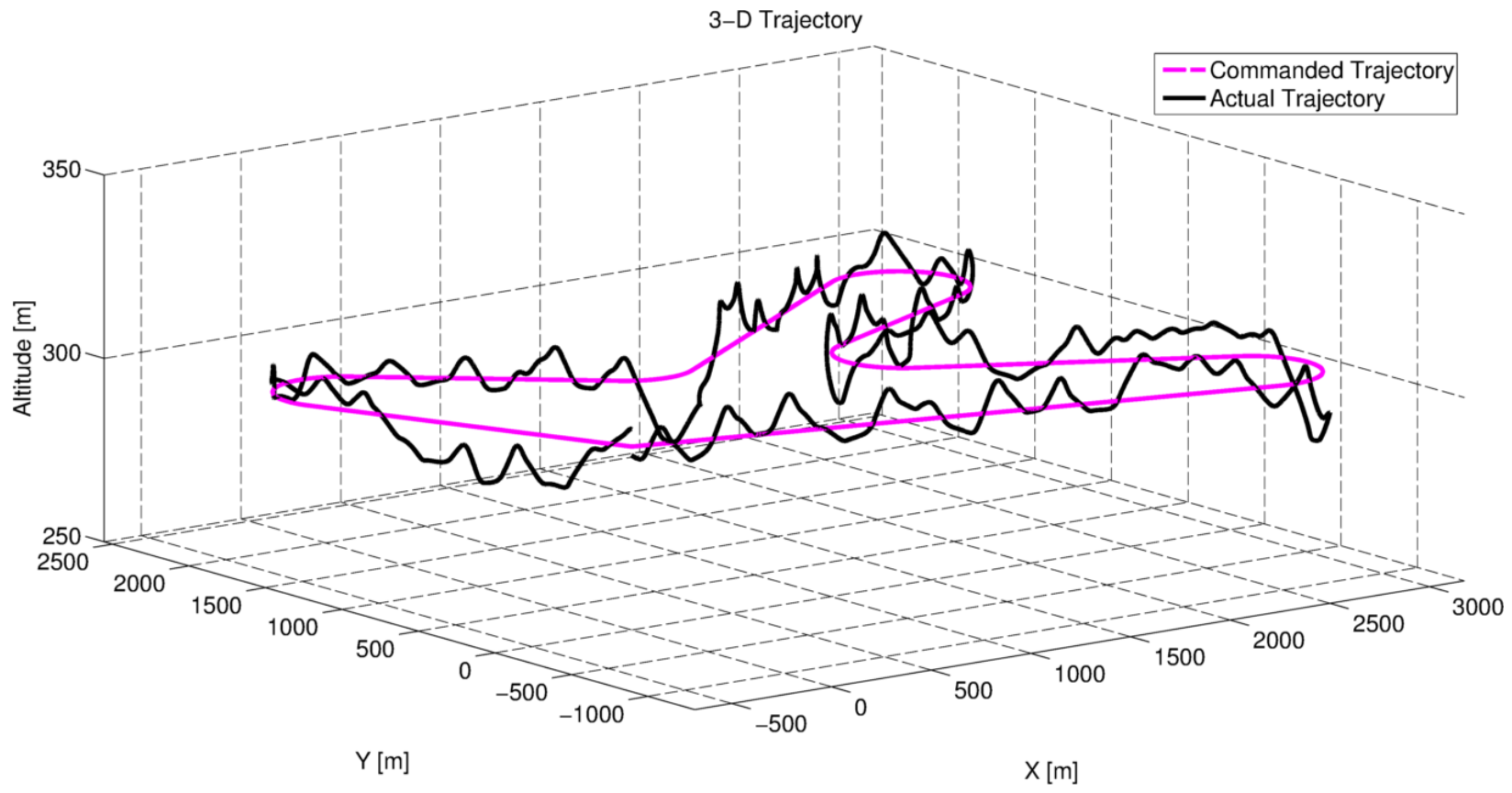

Figure 62: Commanded and Actual Trajectories for Adaptive Position PID at $0.98 \mathrm{sec}$ Velocity Update Interval

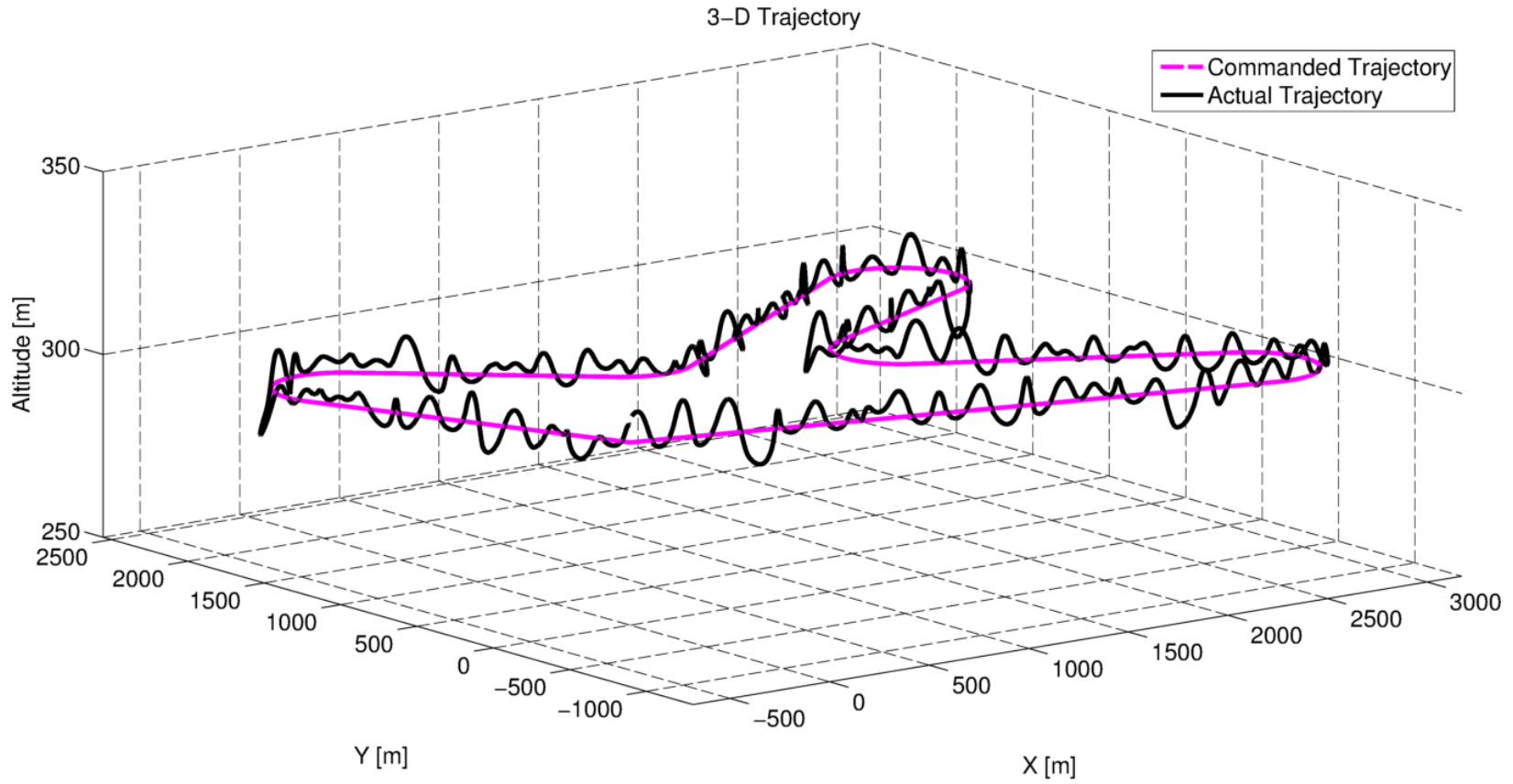

Figure 63: Commanded and Actual Trajectories for NLDI Extended at $0.98 \mathrm{sec}$ Velocity Update Interval 
The controllers' surface deflection for the previous case is presented in Figure 64 and Figure 65.

Stabilator
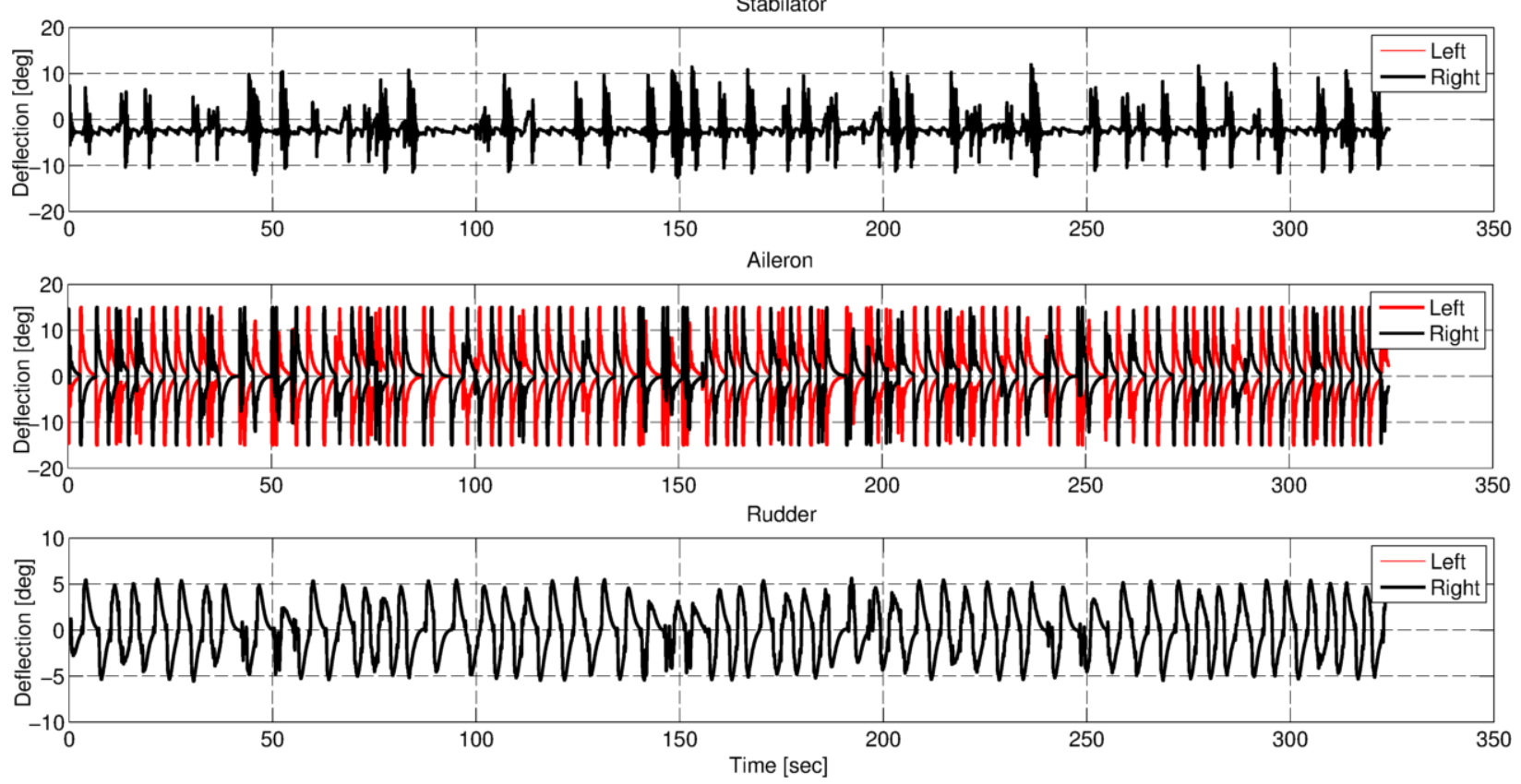

Figure 64: Controller Surface Deflection for Adaptive Position PID at $0.98 \mathrm{sec}$ Velocity Update Interval
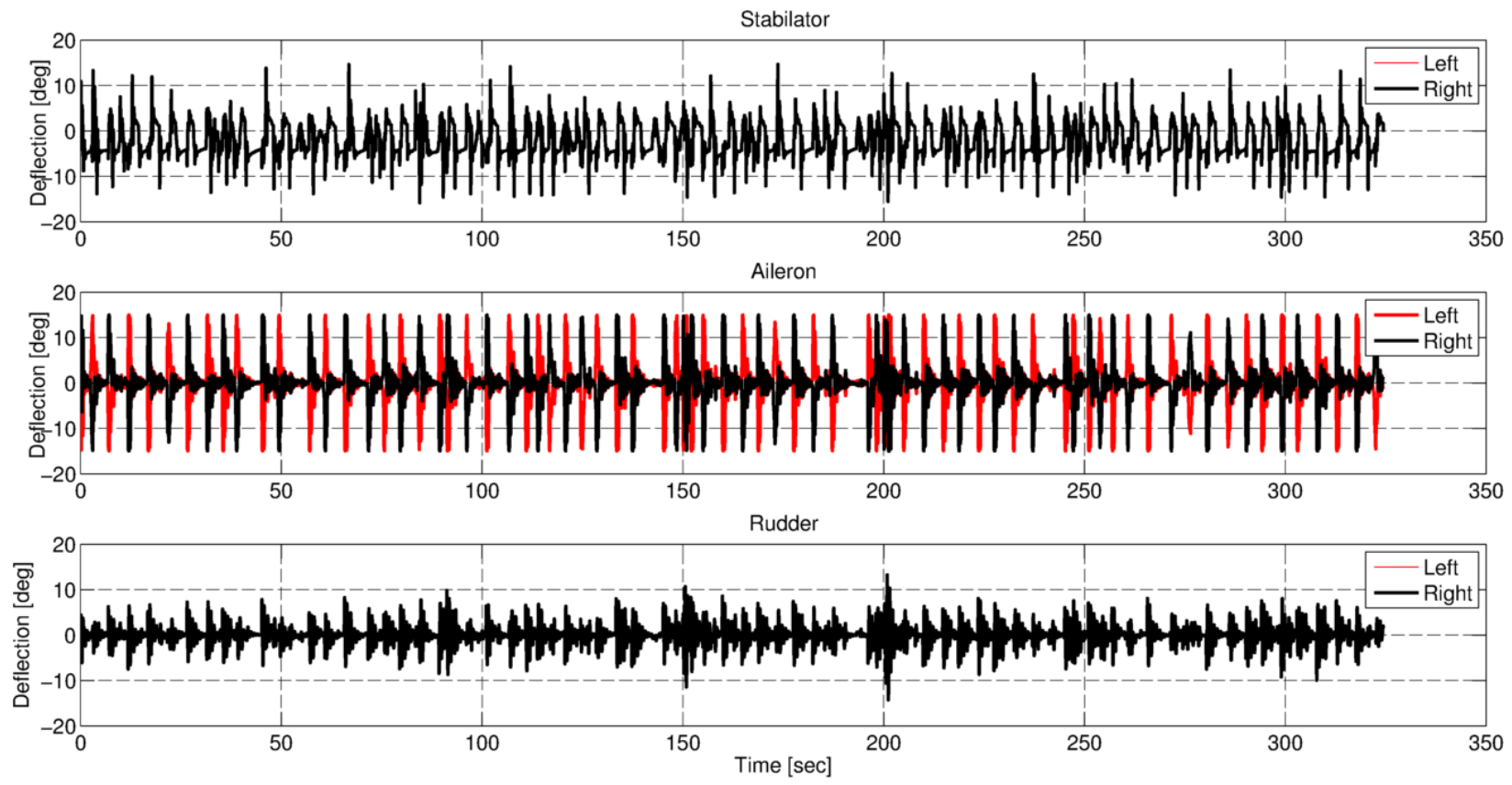

Figure 65: Controller Surface Deflection for NLDI Extended at $0.98 \mathrm{sec}$ Velocity Update Interval 


\subsection{Position and Velocity Error STD Sensitivity Analysis}

In position and velocity error standard deviation sensitivity analysis, the performances of the six controllers were evaluated against different position and velocity errors standard deviation compensations. Table 15 summarize the position and velocity error STD sensitivity analysis.

\begin{tabular}{|c|c|c|}
\hline & $\begin{array}{c}\text { Position Error STD } \\
\text { Start Value: Step: End } \\
\text { Value }\end{array}$ & $\begin{array}{c}\text { Velocity Error STD } \\
\text { Start Value: Step: End } \\
\text { Value }\end{array}$ \\
\hline Figure 66 & $0: 0.25: 4$ & 0 \\
\hline Figure 69 & 0.05 & $0: 0.25: 4$ \\
\hline Figure 72 & $0: 0.25: 4$ & $0.3 *$ Position Error STD \\
\hline Figure 73 & $0: 0.25: 4$ & $0.65 *$ Position Error STD \\
\hline Figure 74 & $0: 0.25: 4$ & Position Error STD \\
\hline
\end{tabular}

Table 15: Position and Velocity Errors STD Analysis Summery

Figure 66 illustrate the controllers' performance in the presence of position noise in the range of 0 and 4 meter $(1 \sigma)$, while Figure 67 and Figure 68 show the controllers' TTI and CAI for this analysis, respectively.

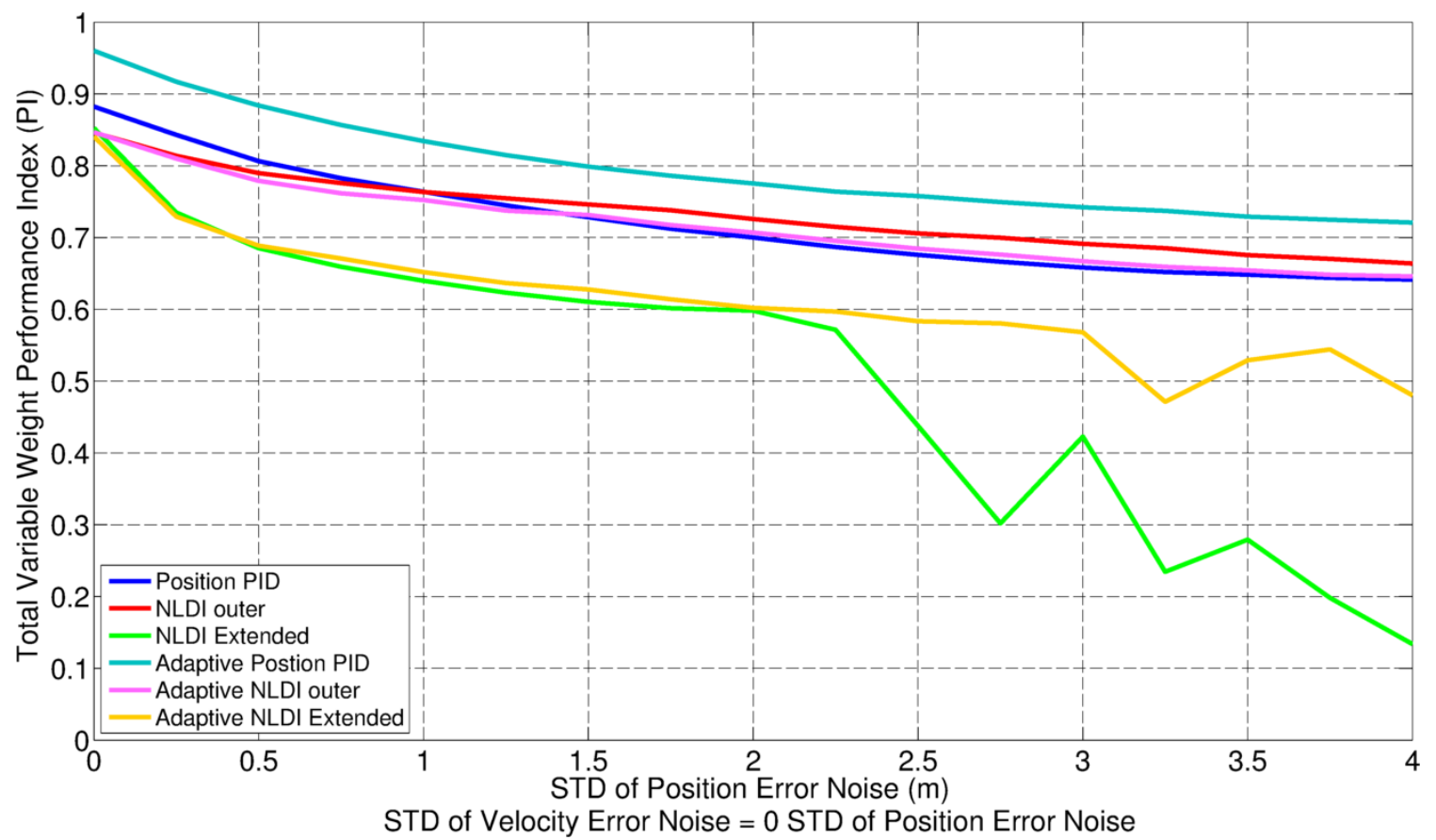

Figure 66: Controllers' Performance Index vs. Position Error STD 


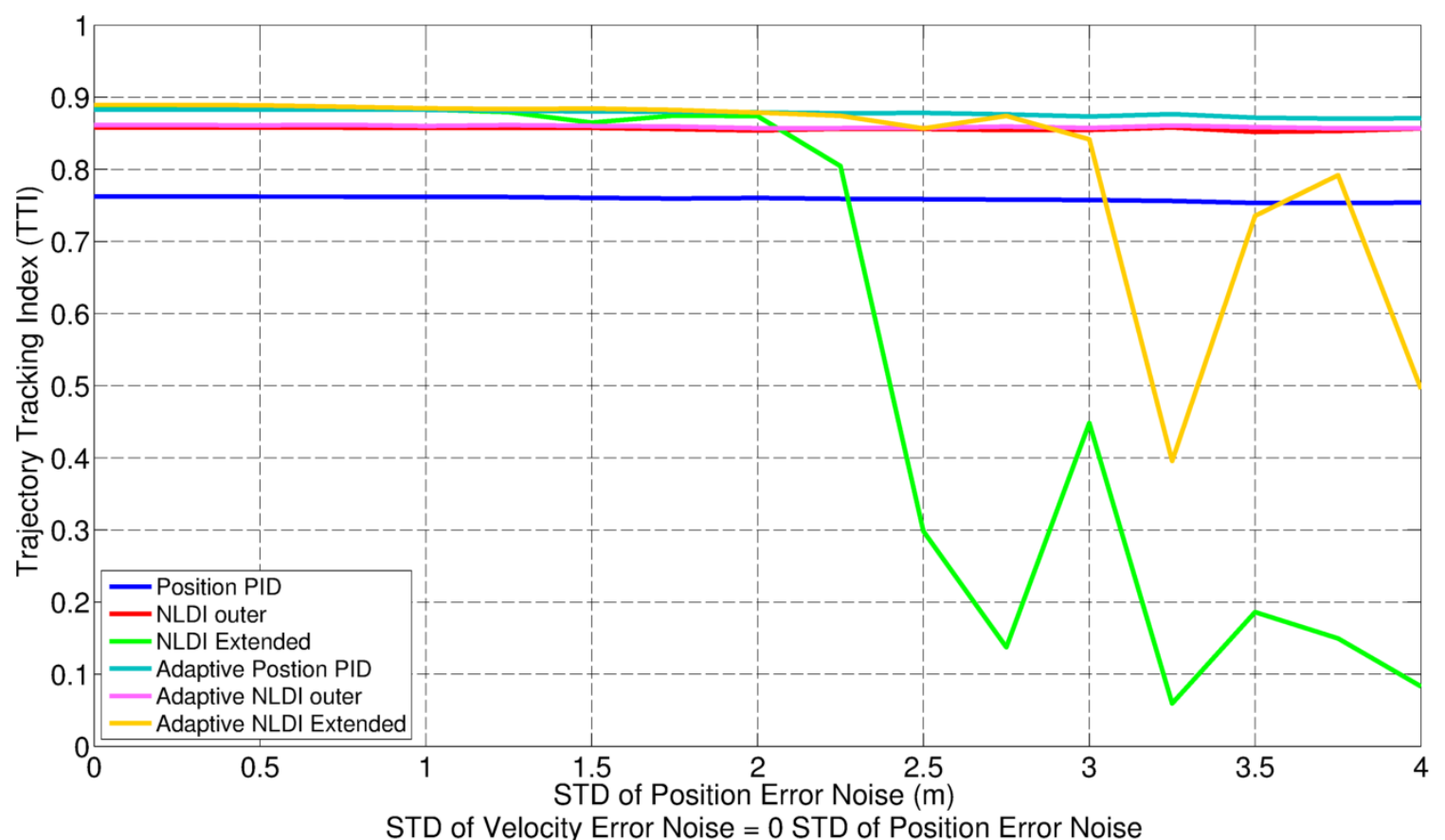

Figure 67: Controllers' TTI Index vs. Position Error STD

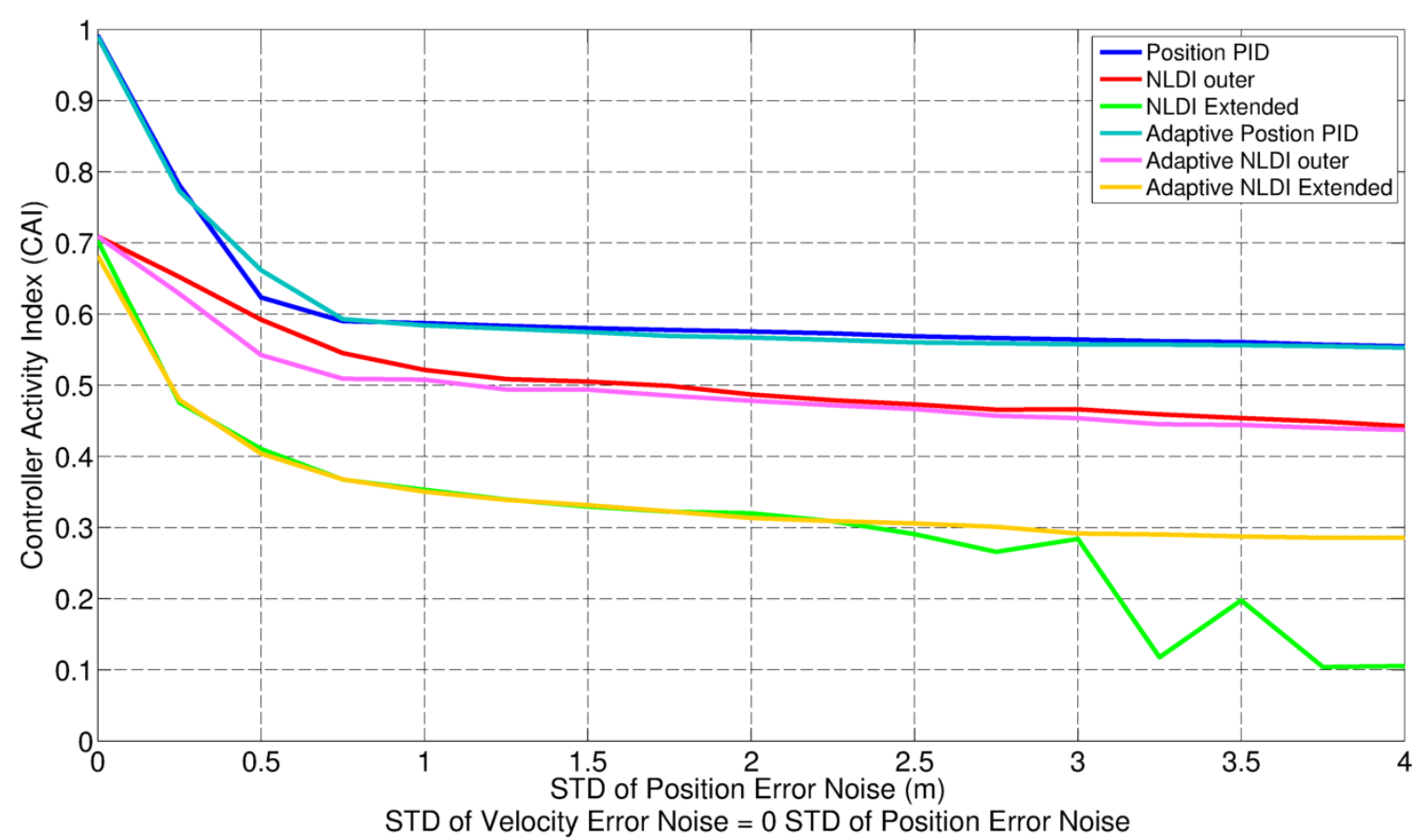

Figure 68: Controllers' CAI Index vs. Position Error STD

The position PID controller appears to be the best performing controller on this category, while the NLDI and its adaptive version share the poorest performing controller position. In fact, the NLDI controller performance goes under 0.4 after 2.5 meter $(1 \sigma)$ of position noise, then it goes up and down for the rest of this analysis. The NLDI extended controller has unpredictable 
behavior to large noises, the controller might success getting the aircraft to the goal point while fails sometimes. The position PID controller performs better than the outer-loop controllers for the first 1 meter STD of position noise; however, the NLDI outer-loop controllers start to perform better than the PID for the higher noise values.

The position error STD does not affect the trajectory tracking index of the well performing controllers (

Figure 67); however, great impact can be seen on the NLDI extended controller after 2 meters and on the adaptive NLDI extended after 3 meters. The position error noise increases the controller activity needed by the controllers to keep the aircraft on the trajectory, as expected (Figure 68). The increase in the controller activity makes the aircraft unstable when flying on the trajectory. The position error noise equally affects the well performing controllers and their adaptive version about the same, while the adaptive NLDI shows a better noise robustness than its conventional counterpart.

The second analysis in this category is the study of the velocity error STD effect on the controller's trajectory tracking performance. In this analysis, the velocity error STD was increased from 0 to 4 meter per second $(1 \sigma)$ in 0.25 meter per second increments while having a 0.05 STD position noise. This combination of position and velocity noise might not exist in a real GPS receiver since GPS velocity accuracy is better than the position accuracy. However, this analysis is meant to study the isolated effect of pure velocity noise on the controllers' performances. Figure 69 overviews the controllers' performance under these circumstances. Figure 70 shows the controllers' TTI, while Figure 71 displays the controllers' CAI. 


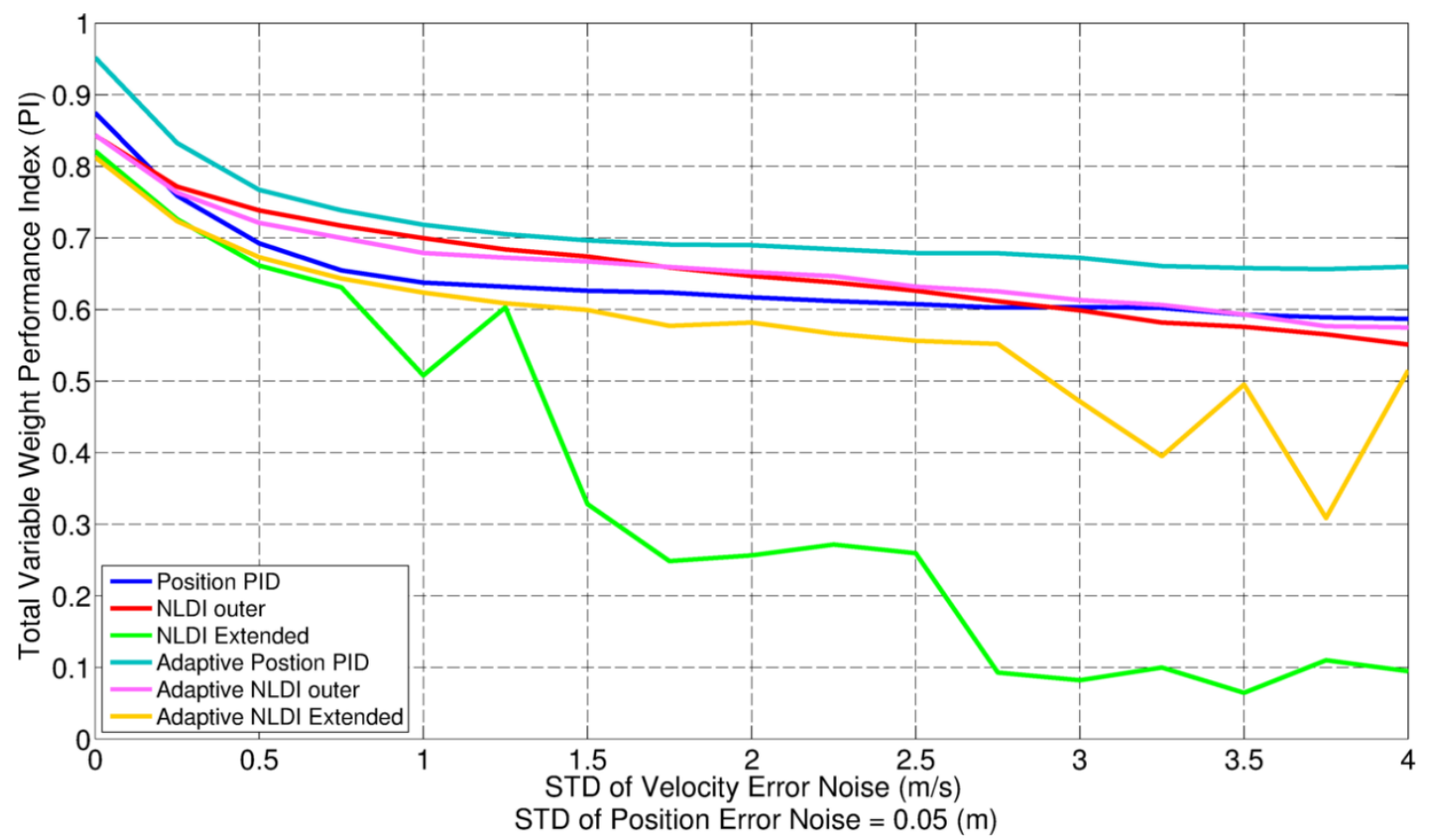

Figure 69: Controllers' Performance Index vs. Velocity Error STD

The velocity error noise has a greater impact on the controllers' performance than the position error noise. The adaptive PID controller has the highest robustness with respect to this parameter. The outer NLDI controller and its adaptive version achieve better performance index than the position PID for STD values lower than 0.25 meter per seconds, while the extended controller stays at the poorest performing level, and it fails to get the aircraft to the finish point for STD values larger than 1.5 meter per seconds. However, the adaptive NLDI extended controller has a better robustness to the velocity noise and it tolerant an STD value of $3 \mathrm{~m} / \mathrm{s}$. 


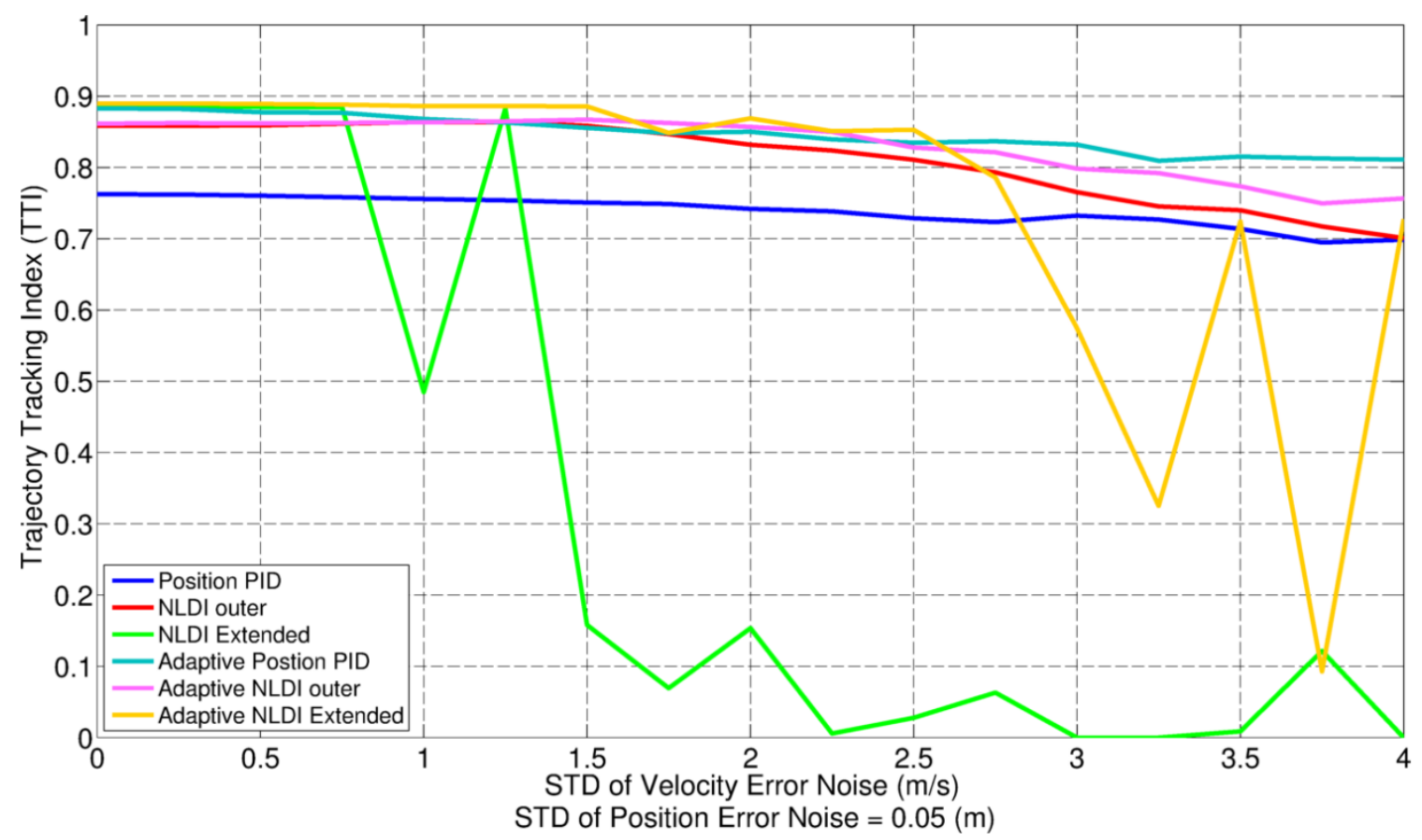

Figure 70: Controllers' TTI Index vs. Velocity Error STD

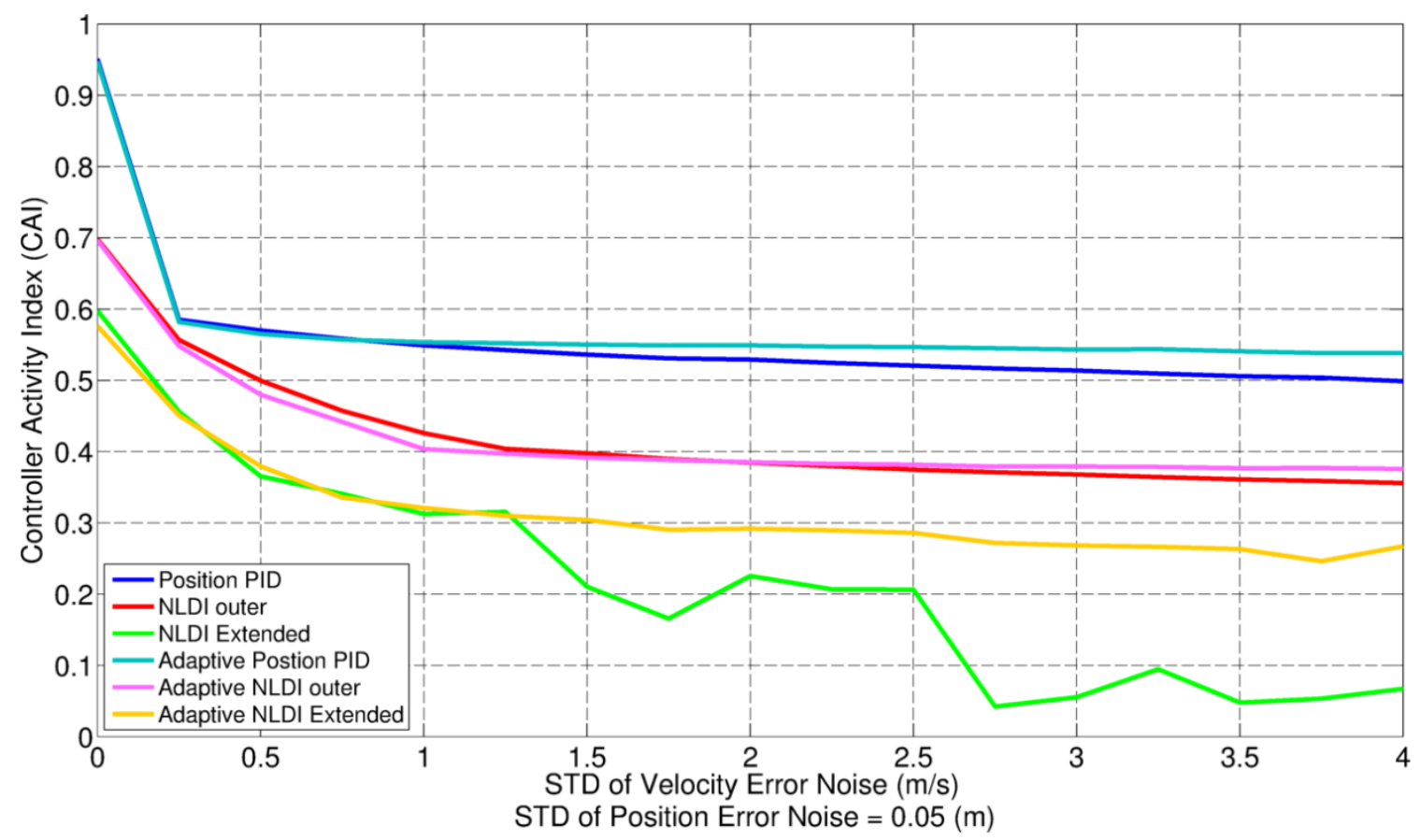

Figure 71: Controllers' CAI Index vs. Velocity Error STD

A close look to the controllers' trajectory tracking index shows that the velocity noise affects it to lower extent for the well performing controllers; however, the NLDI controllers' TTI shows more pronounced sensitivity to the velocity noise. The controllers' CAI shows the NLDI extended 
controller's index going below 0.2 at 1.5 meters per seconds STD of velocity noise due primarily to surface deflection saturation.

The last set of tests in the position and velocity noise category consists of three cases. These analyses test the controllers against three different position and velocity error noise combinations. The STD of the position error noise varies from 0 to 4 in all analyses. The STD of the velocity error noise is $30 \%$ of the STD of position error noise in the first analysis (see Figure 72 ), and it is $65 \%$ of the STD of position error noise in the second analysis (see Figure 73). Finally, position and velocity error have the same STD on the last analysis (see Figure 74). The controllers' TTI and CAI are not shown because they exhibit the same trend as in the two previous analyses, that is a rather reduced effect of STD on the TTI of the well performing controllers' and a more important impact on the poorest performing controllers and the CAI. The results show the same performance trends for all controllers. The higher the level of the position and velocity noise STD, the worse the degradation of the controllers' performance. However, generally, the controllers show better robustness with respect to this parameter as compared to the others investigated so far.

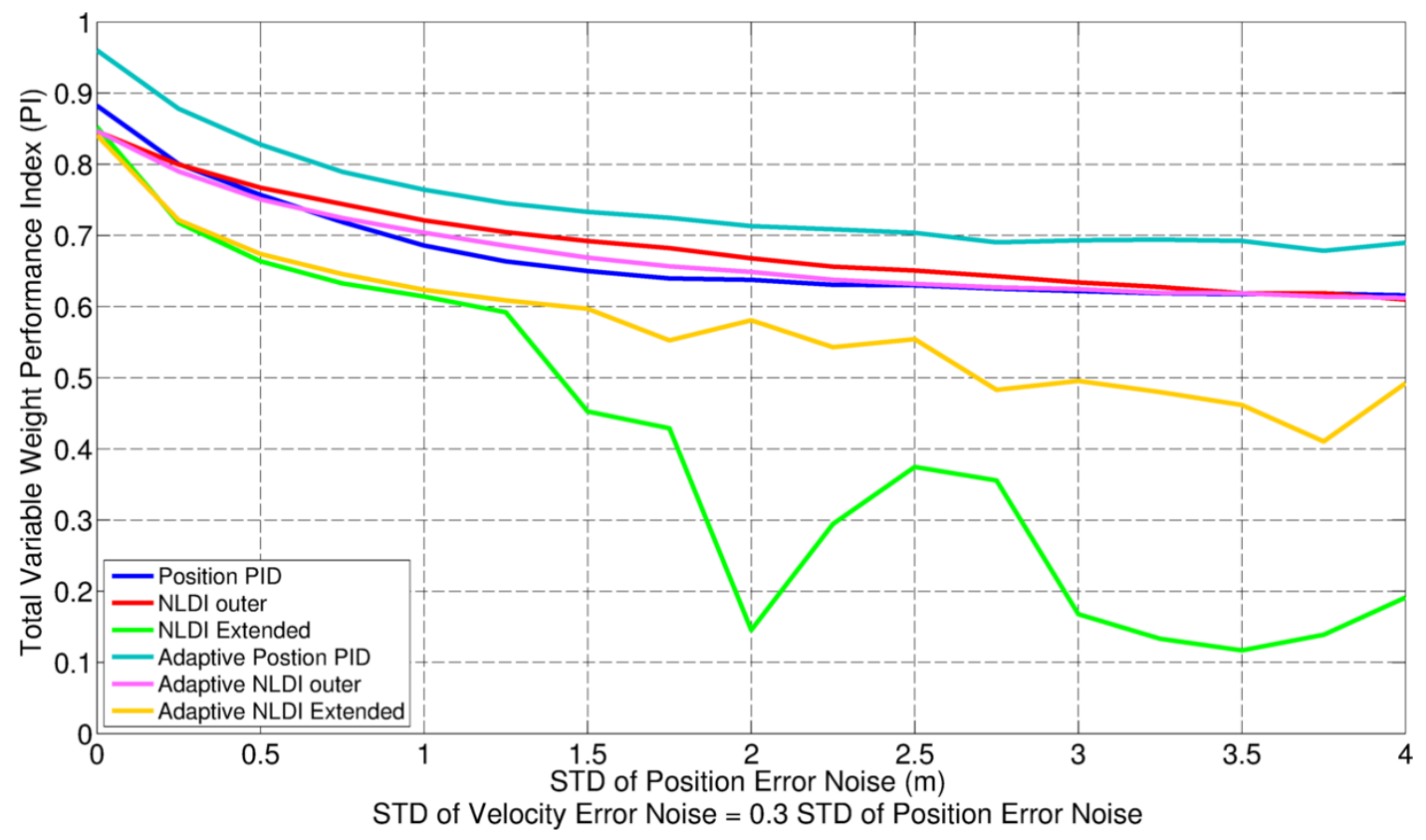

Figure 72: Controllers' Performance Index vs. Position and Velocity Error STD (Case 1) 


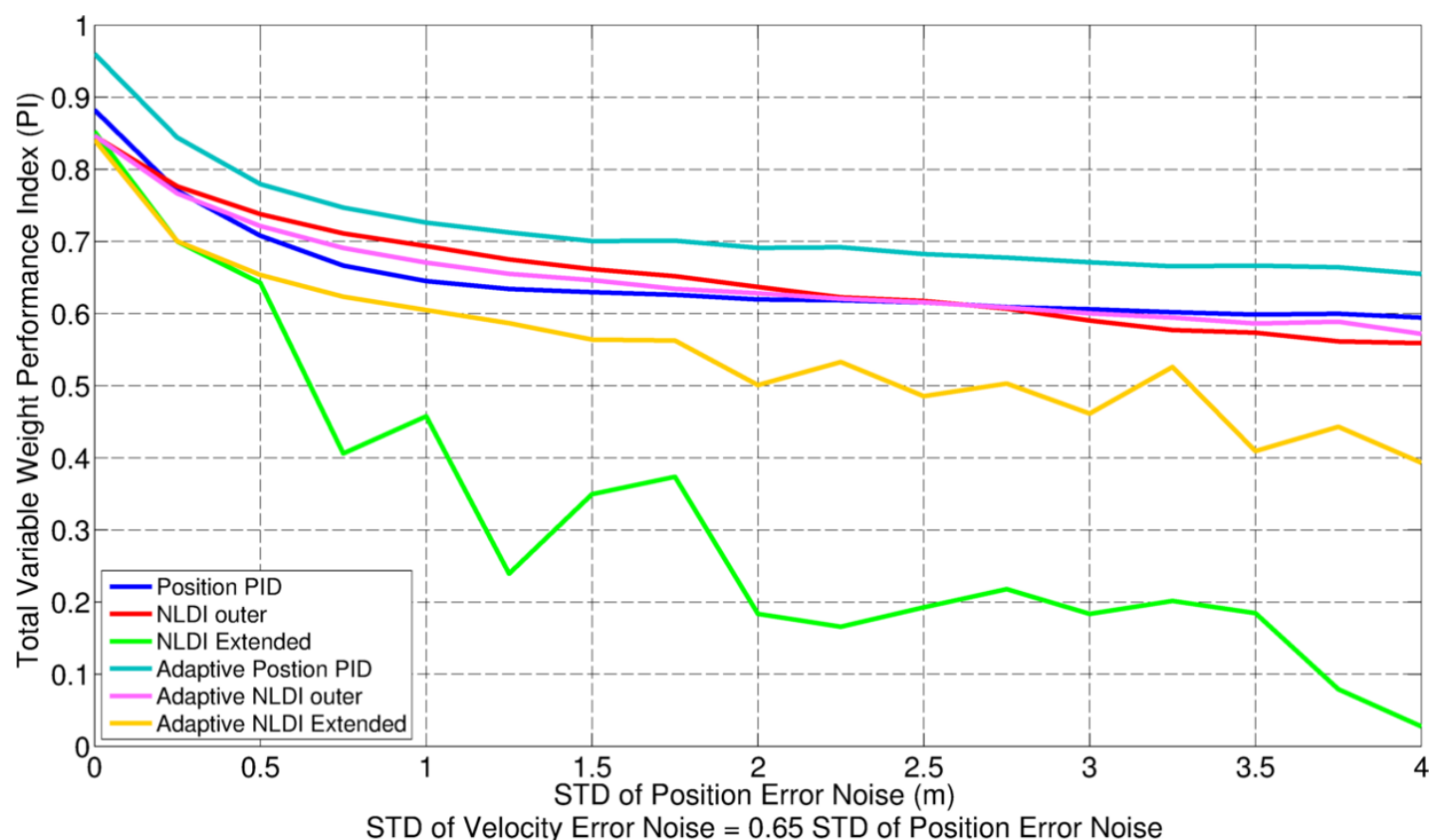

Figure 73: Controllers' Performance Index vs. Position and Velocity Error STD (Case 2)

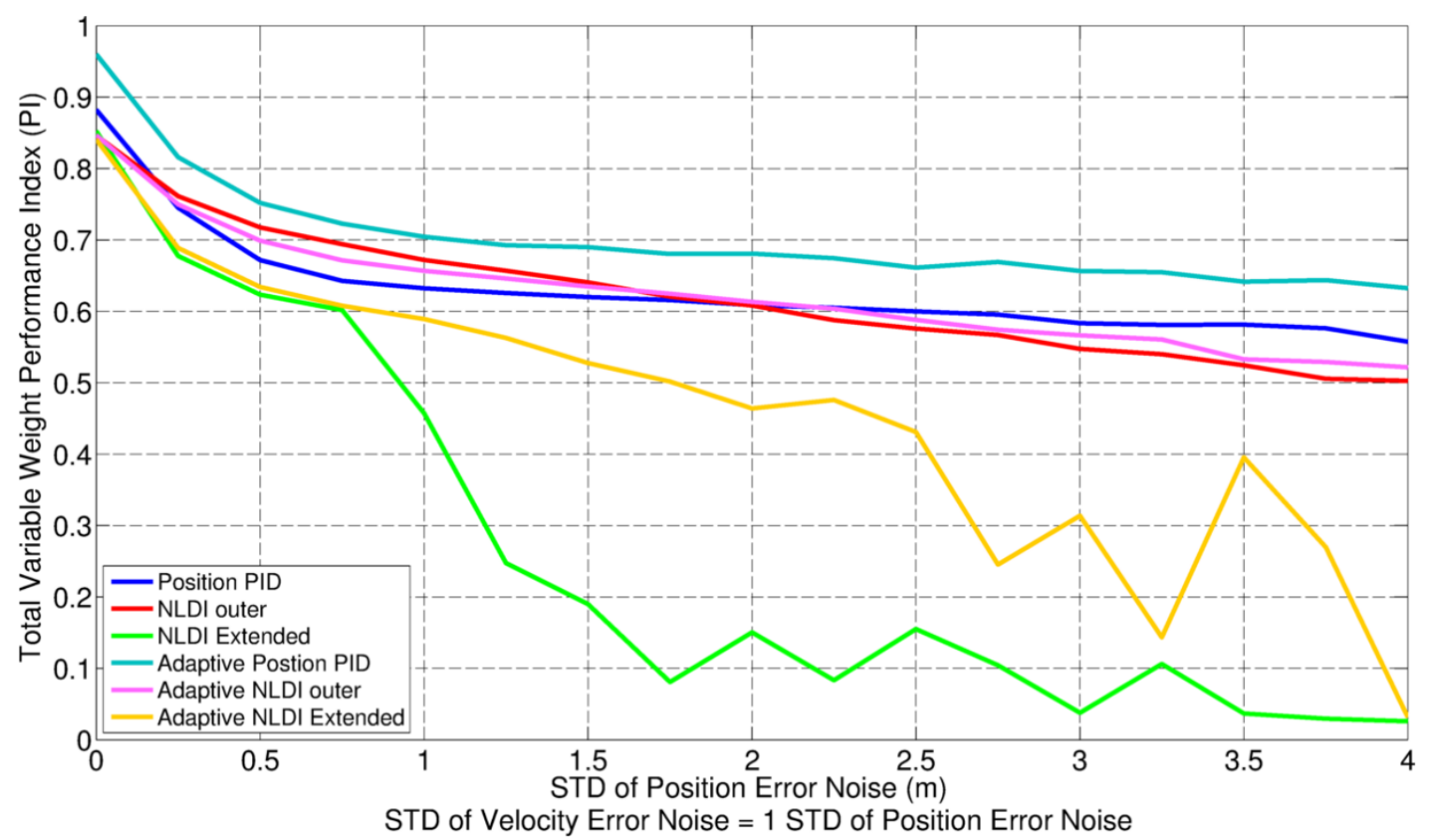

Figure 74: Controllers' Performance Index vs. Position and Velocity Error STD (Case 3)

As a summary of the STD of position and velocity error noise analysis, it can be stated that the NLDI extended controllers have good robustness with respect to only small levels of measurement noise $($ STD<0.5). The position PID and the outer-loop controllers have good 
robustness with respect to measurement noise and they can tolerate a combination of $4 \mathrm{~m}$ and $4 \mathrm{~m} / \mathrm{s}$ STD of position and velocity error noise.

An example of actual and commanded trajectories for both adaptive position PID and the NLDI extended controllers are shown in Figure 75 and Figure 76 respectively. These results are obtained for values of $2 \mathrm{~m}$ position noise STD and 0.6 second/s velocity noise STD.

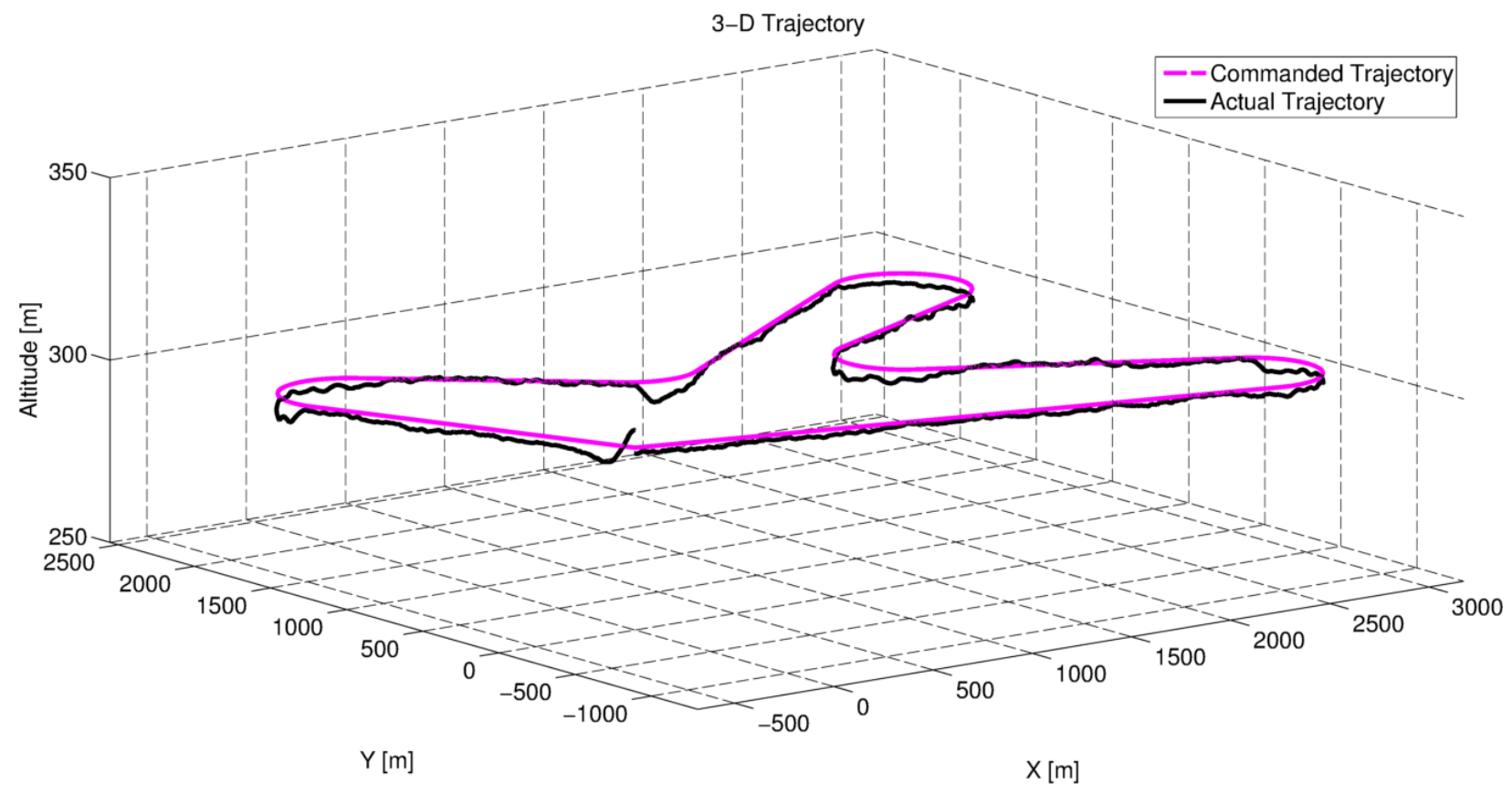

Figure 75: Commanded and Actual Trajectories for Adaptive Position PID at 2 (m) STD Position Noise, and $0.6(\mathrm{~m} / \mathrm{s})$ STD Velocity Noise 


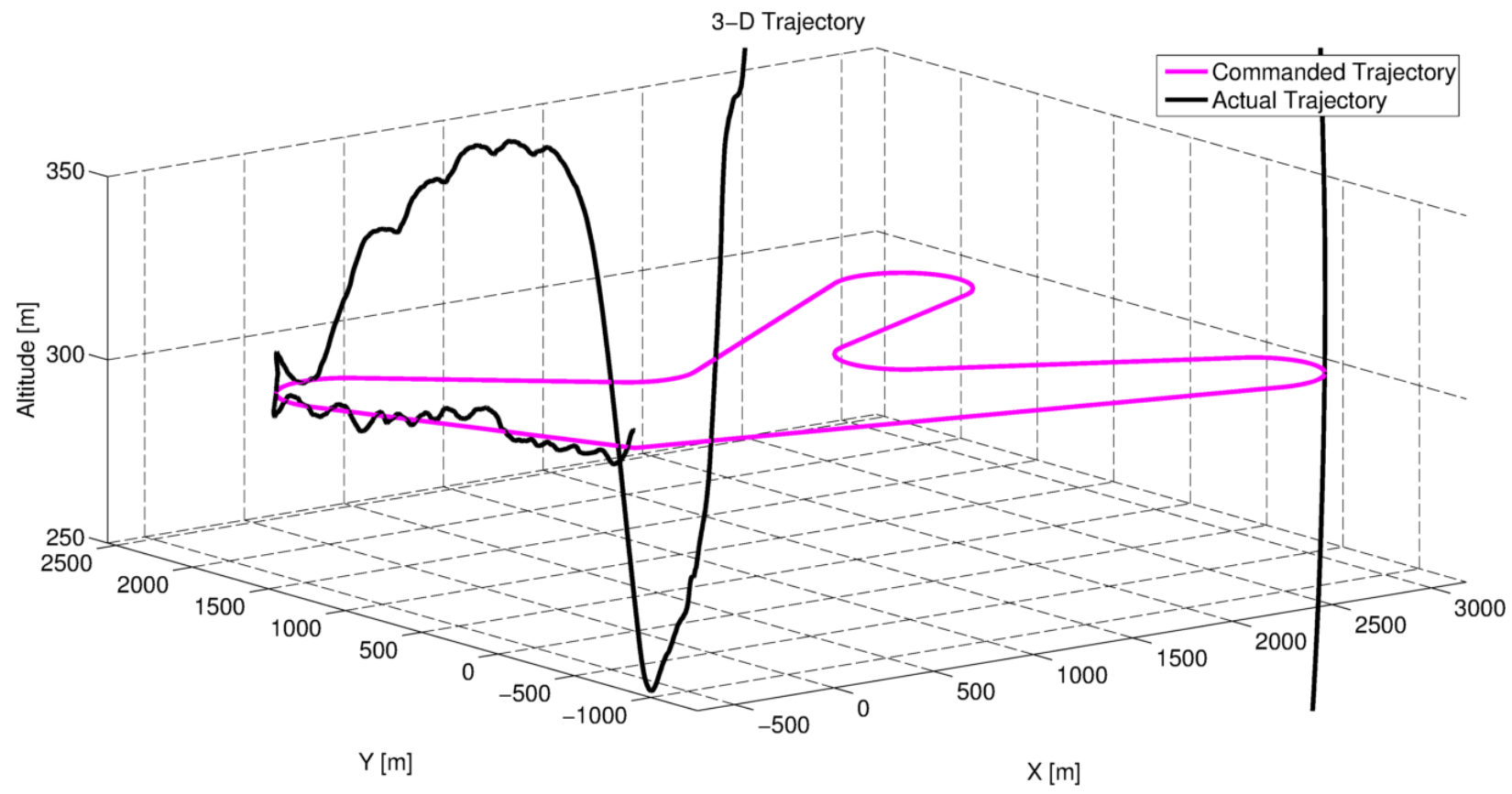

Figure 76: Commanded and Actual Trajectories for NLDI Extended at $2(\mathrm{~m})$ STD Position Noise, and 0.6(m/s) STD Velocity Noise
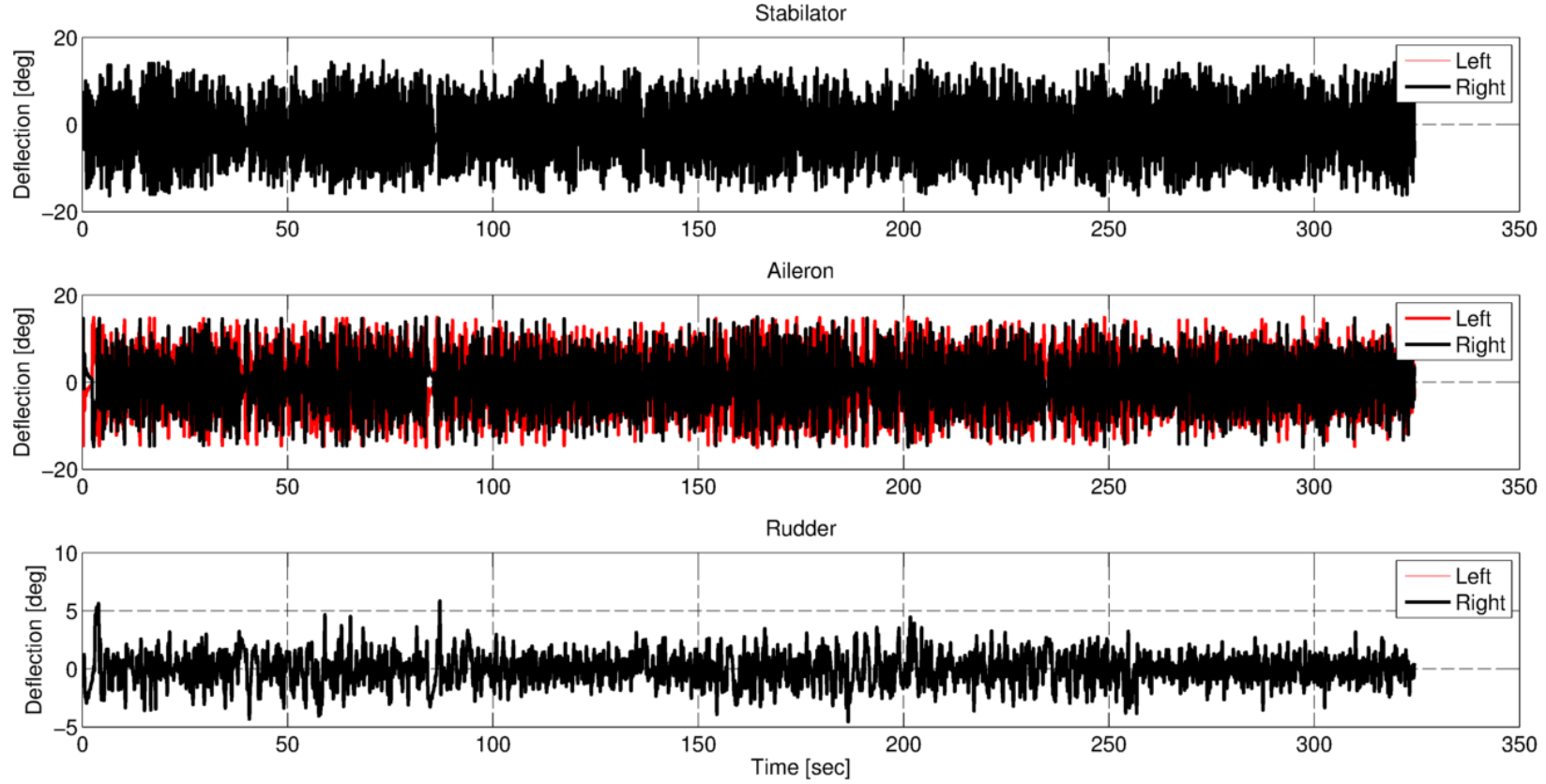

Figure 77: Controller Surface Deflection for Adaptive Position PID at $2(\mathrm{~m})$ STD Position Noise, and 0.6(m/s) STD Velocity Noise 

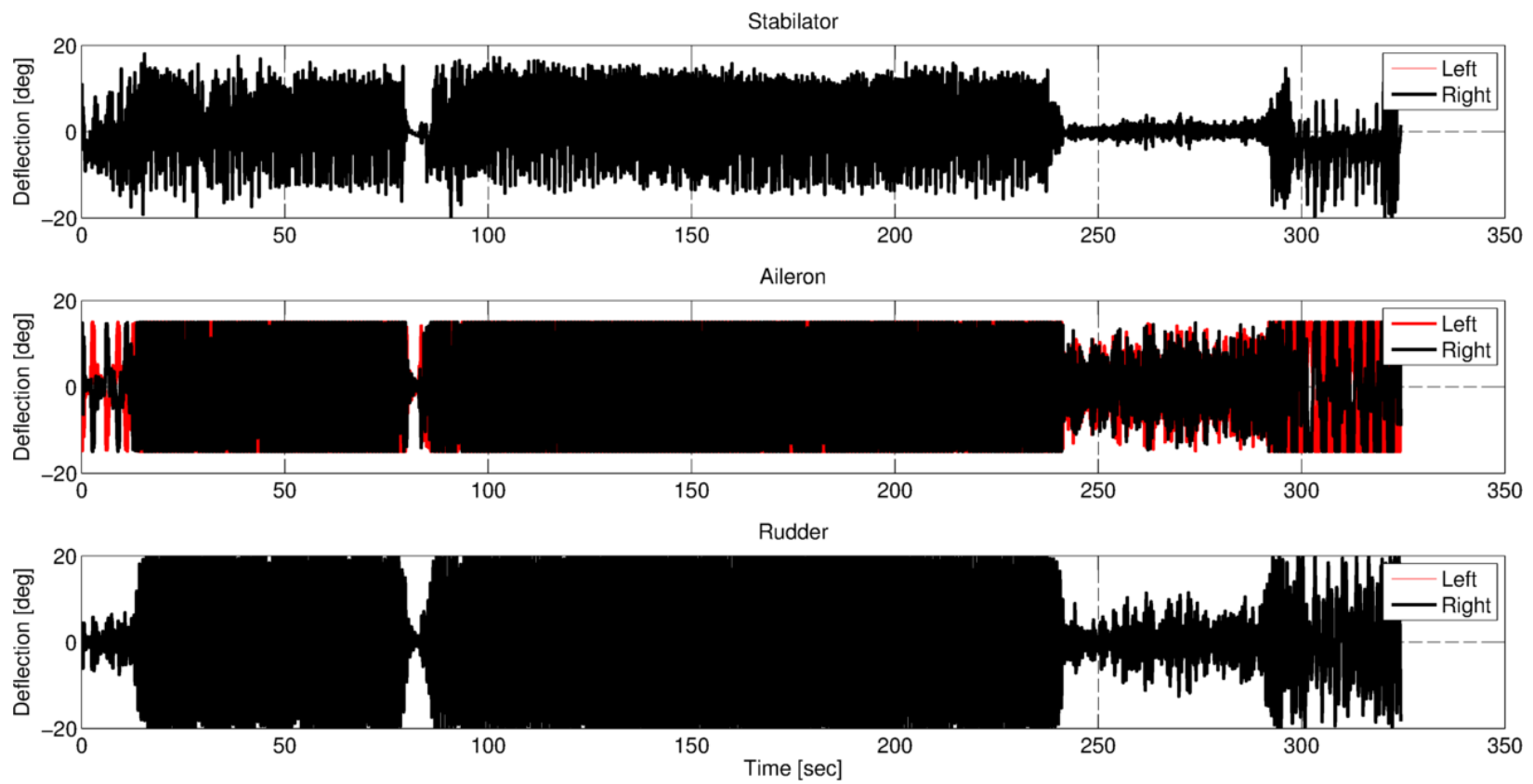

Figure 78: Controller Surface Deflection for NLDI Extended at $2(\mathrm{~m})$ STD Position Noise, and $0.6(\mathrm{~m} / \mathrm{s})$ STD Velocity Noise

\subsection{GPS Failure Modes Analysis}

According to the GPS failure mode analysis presented in section 3.9, the GPS integrity failures might introduce excessive noise, step, ramp, or sinusoidal errors in the URE measurement. At the same time, it will introduce the time derivative of URE failure to the URRE. In other words, the GPS integrity failures will affect both position and velocity measurements.

\subsubsection{Noise Sensitivity Analysis}

Excessive noise is one of the GPS integrity failure modes. For example, MCS Upload Error could lead to a noise error value of 13.7 meters $(1 \sigma)$; thus a set of tests were perform to study the effect of GPS position and velocity measurement excessive noise on the controllers' performance. The first set of tests represents the controllers' performances in the presence of position and velocity noise. The position error STD in each test ranges between 2 and 10 meters with increments of 2 meters, while the velocity error measurement is the time derivative of the position error. The noise failure was tested for durations of 12, 25, 50, and 75 seconds. Figures (57) through (60) present the performance results. 


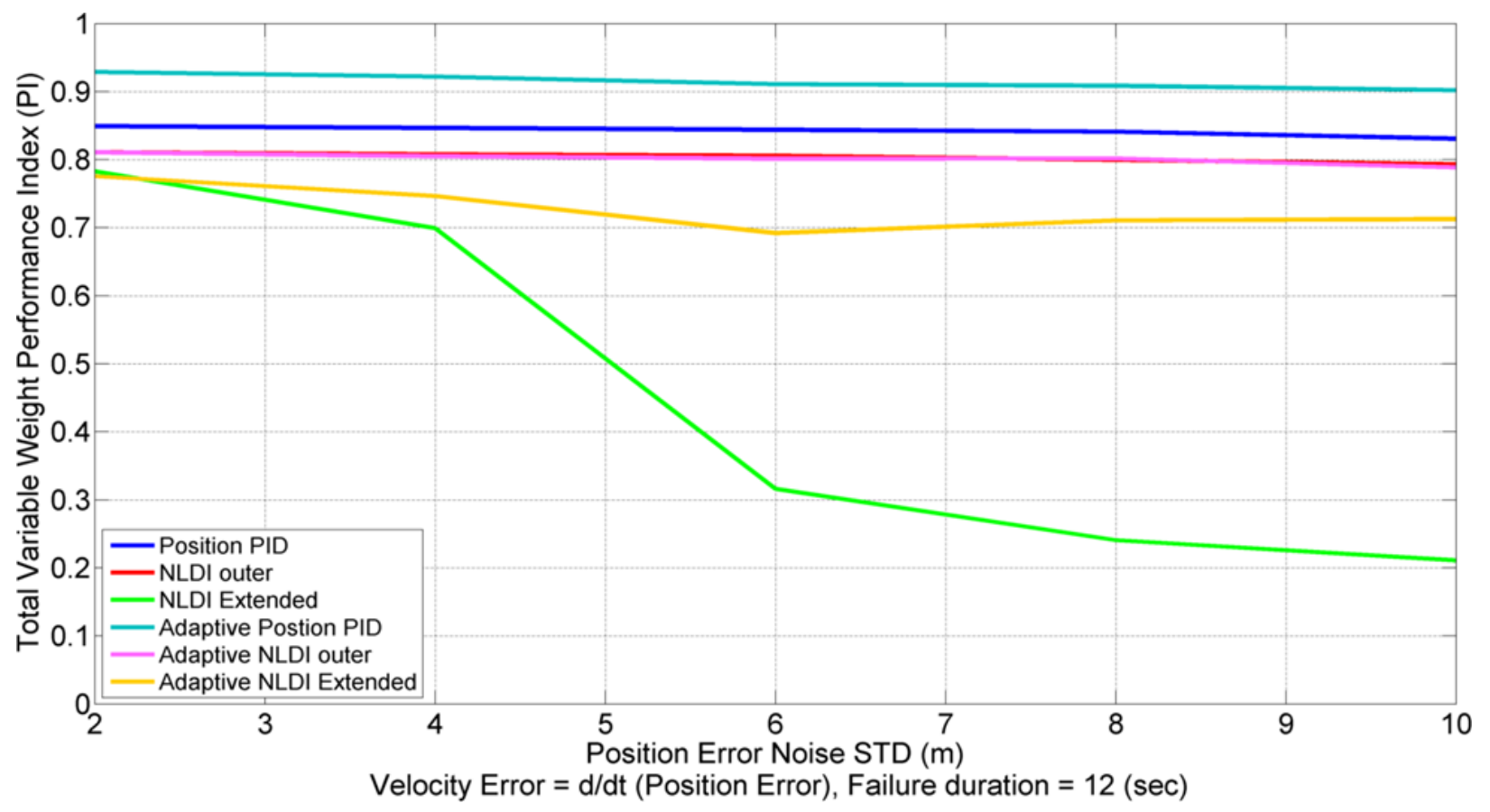

Figure 79: Controllers' Performance Index vs. Measurement Noise for $12 \mathrm{sec}$

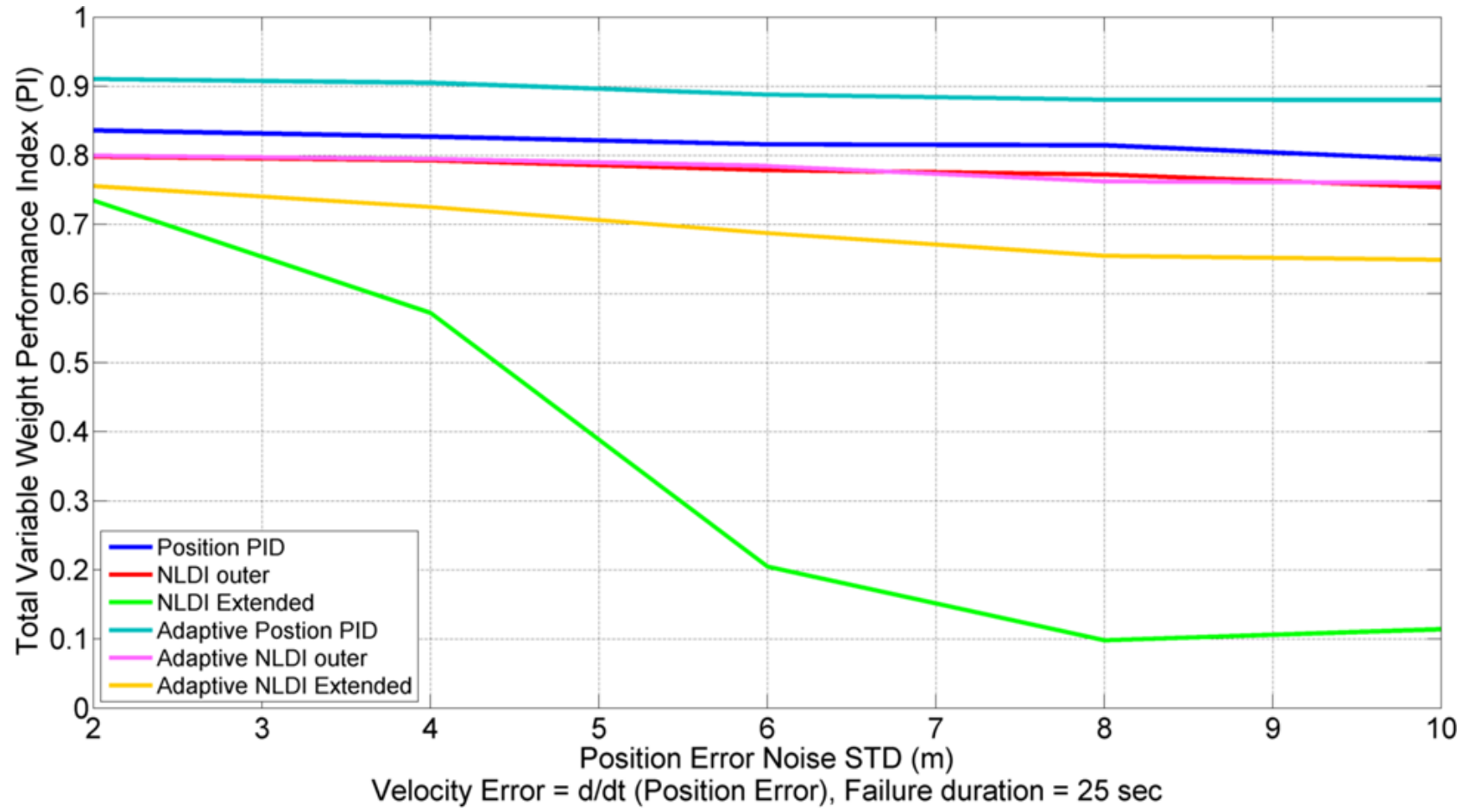

Figure 80: Controllers' Performance Index vs. Measurement Noise for $25 \mathrm{sec}$ 


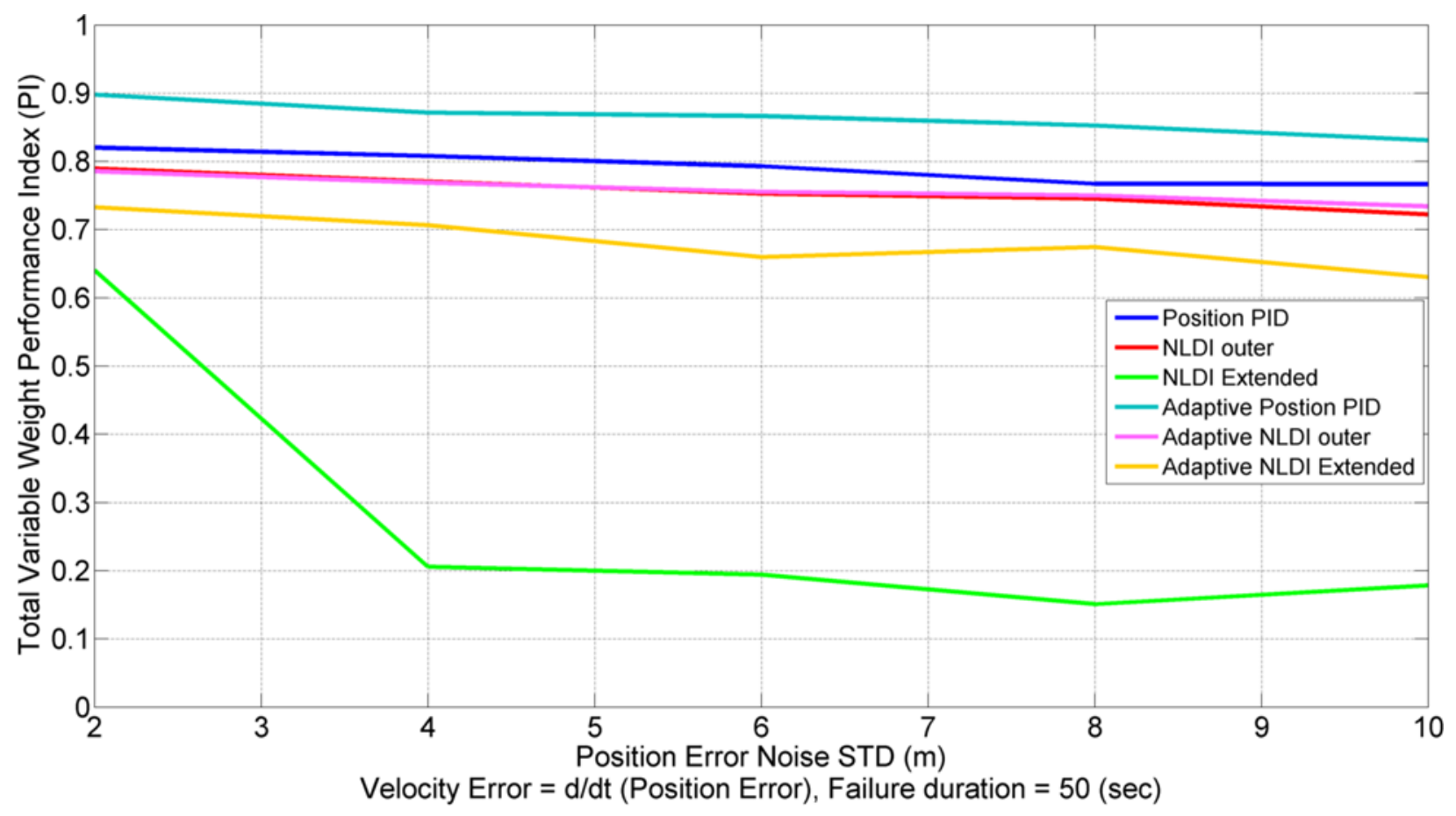

Figure 81: Controllers' Performance Index vs. Measurement Noise for $50 \mathrm{sec}$

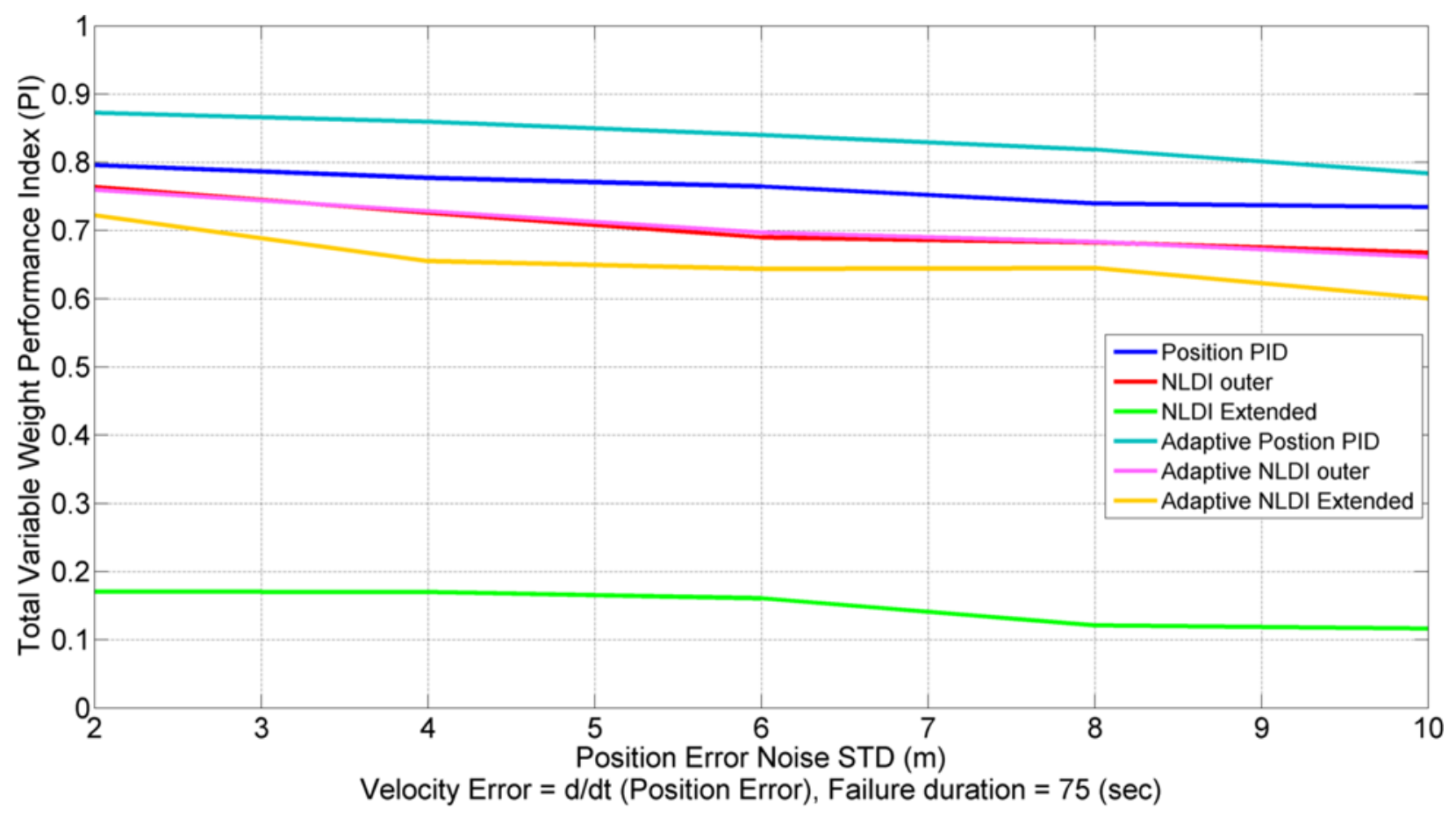

Figure 82: Controllers' Performance Index vs. Measurement Noise for $75 \mathrm{sec}$

As seen in the above figures, the noise failure generally has little impact on the performance of the controllers. The PI decreases slightly as the noise magnitude increases. However, the failure has a larger impact on the performance of the extended NLDI controller. With the extended 
NLDI, the aircraft only survives if the noise is small in magnitude and time duration. For example, the aircraft crashed after having 6 meters standard deviation of the position error for only 12 seconds. The extended NLDI crashes sooner as the standard deviation of the noise increases. In this particular case, the adaptive NLDI controller is more robust to measurement noise than the

fixed-parameter version. The aircraft survives all the tests even though it was the second worst performing controller.

\subsubsection{Step Sensitivity Analysis}

Since the step, ramp, and the sinusoidal will directly impact the trajectory tracking errors, the algorithm used to calculate the controllers' performances from this section on was the fixed weight algorithm. The fixed weight algorithm will emphasize the trajectory tracking error effect introduced by the failures. Step, ramp, and sinusoidal analysis was performed for $12,25,50$, and 75 second duration; however, in the normal operation an alert should be send by the GPS system to the receiver within 12 seconds of the failure occurrence. All the abnormal conditions analyses were performed mainly to test the controllers' ability to recover the aircraft after an abnormal condition occurred.

The step sensitivity analysis starts with a 12 seconds failure duration. The position step values are $10,25,50,75$, and 100 meter for this analysis category, while the velocity experiences an impulse error. Figure 83 shows the controllers' performances in the presence of step failure for 12 second duration, while Figure 84 and Figure 85 display the controllers' TTI and CAI, respectively. 


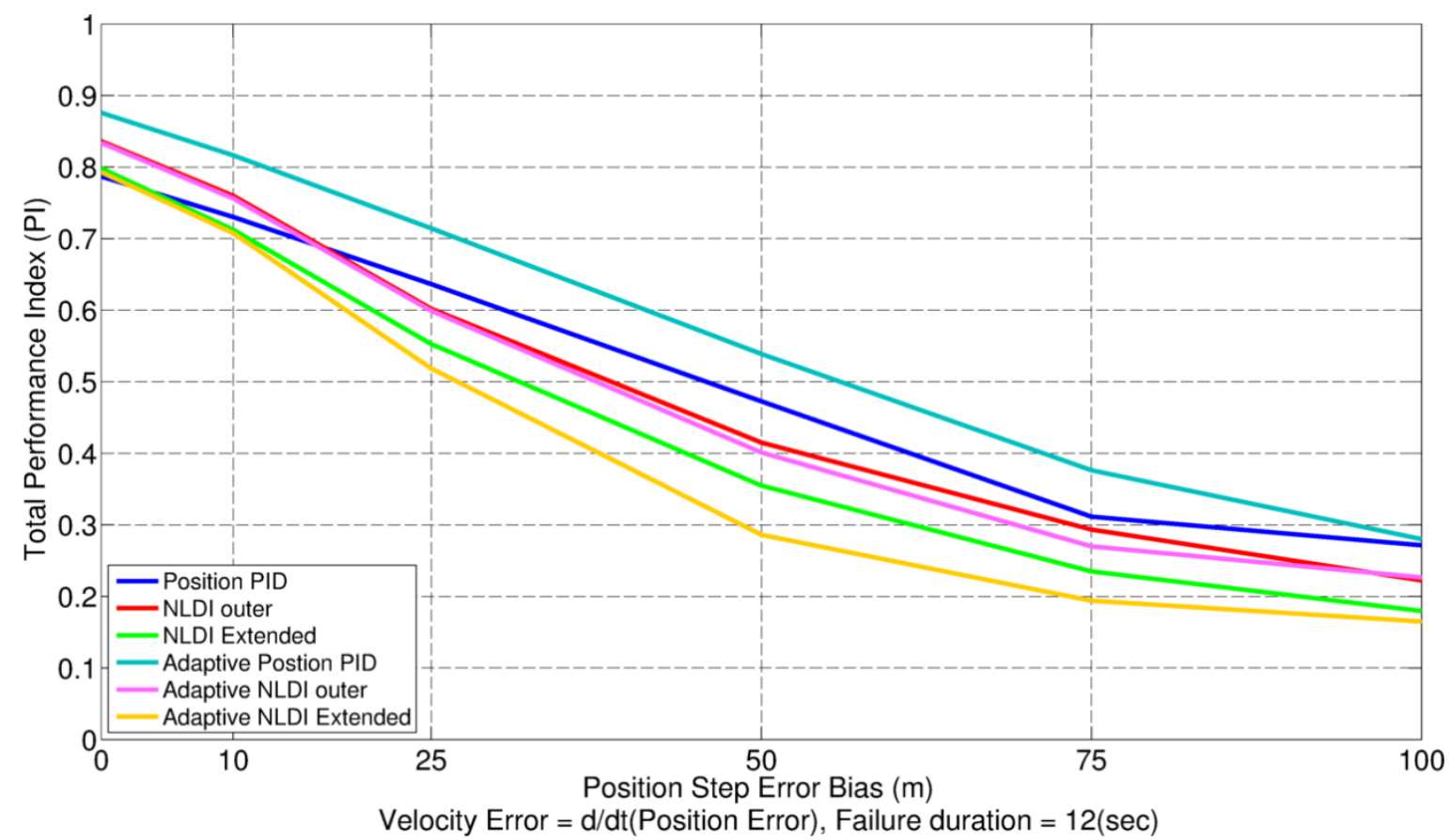

Figure 83: Controllers' Performance Index vs. Measurement Bias for $12 \mathrm{sec}$

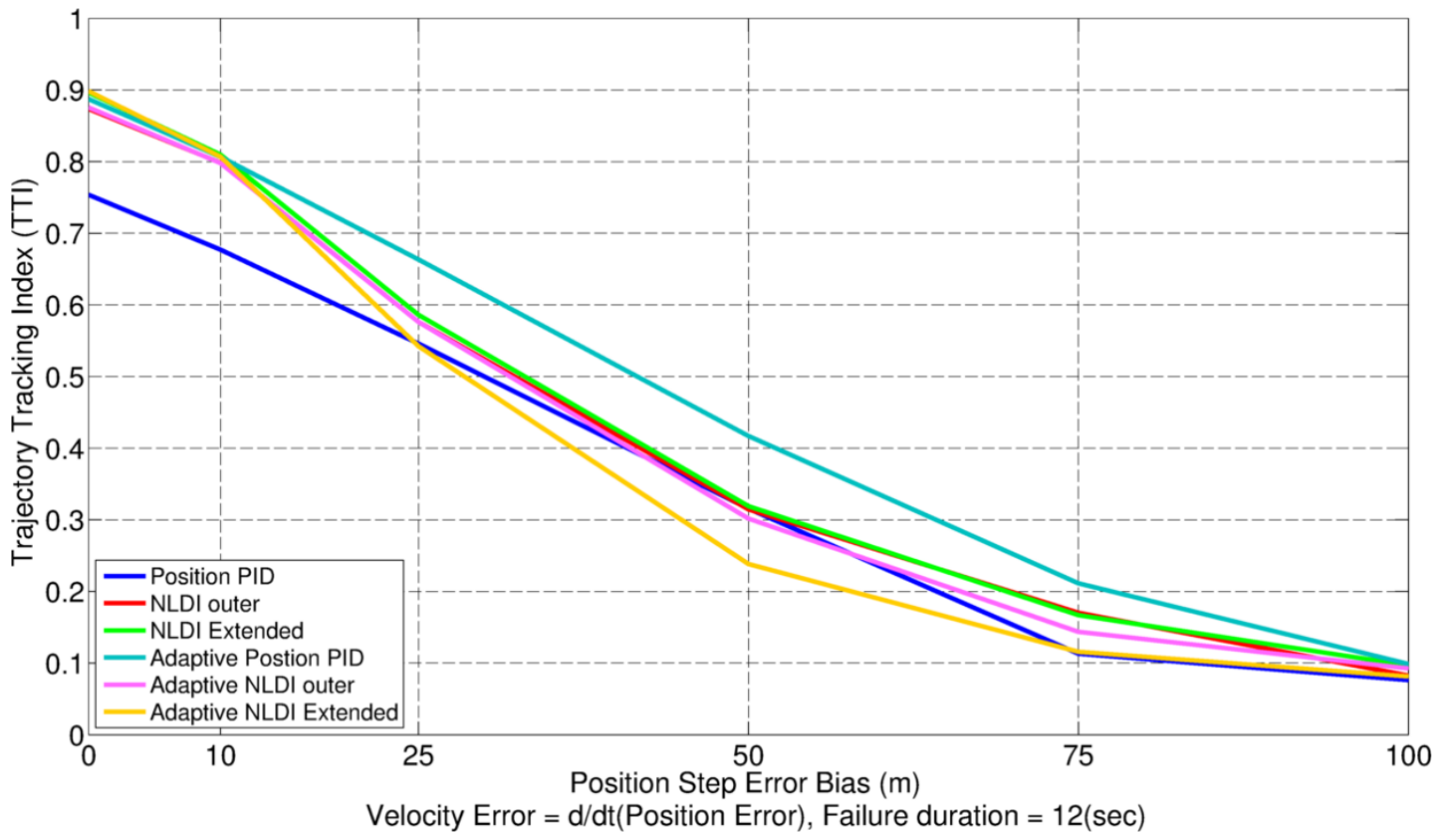

Figure 84: Controllers' TTI vs. Measurement Bias for $12 \mathrm{sec}$ 


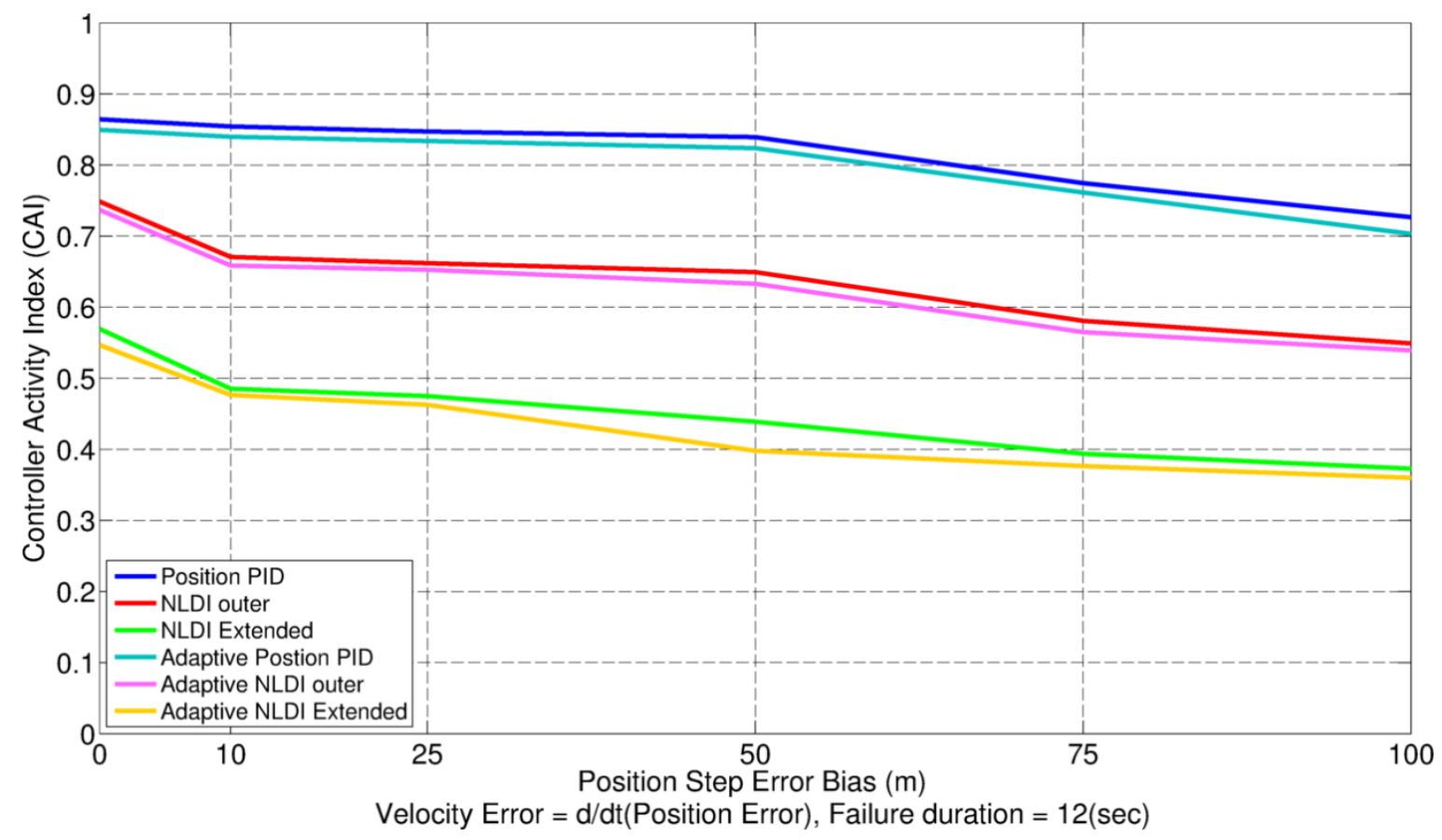

Figure 85: Controllers' CAI vs. Measurement Bias for $12 \mathrm{sec}$

The effect of the bias on the controllers' performance appears to be as anticipated, great impact on the trajectory tracking indices, with a slight effect on the controllers' activities. The adaptive position PID was the best performing controller in this analysis, since it has the best TTI and high CAl, while the adaptive NLDI extended was the poorest performing controller since the step failure has a great impact on its TTI.

The results for the failures with durations of 25,50 , and 75 seconds are presented in Figure 86, Figure 87 , and Figure 88 , respectively. In all these cases the impact of the step failures was mainly on the trajectory tracking error with a slight effect on the controllers' activity. 


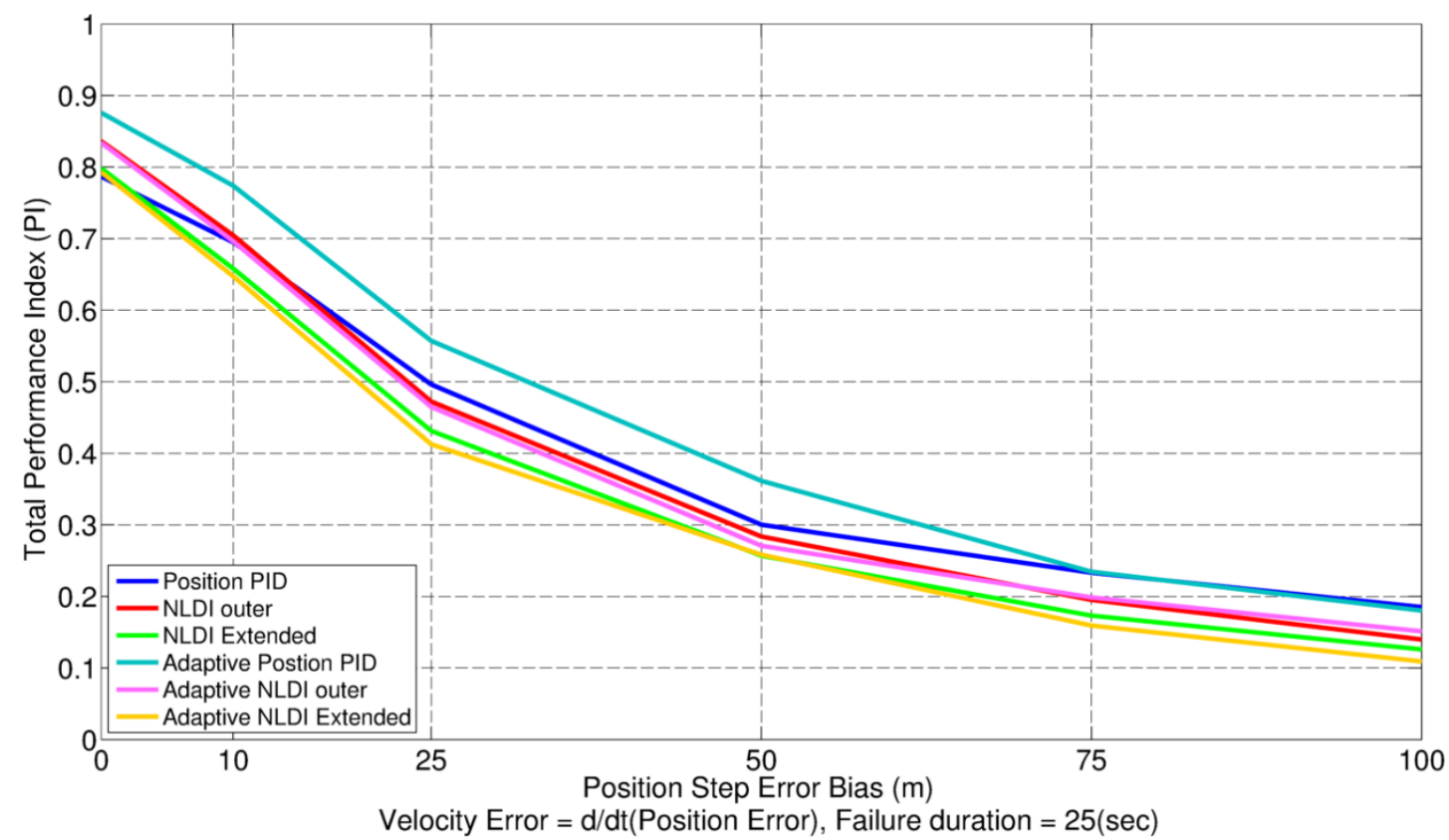

Figure 86: Controllers' Performance Index vs. Measurement Bias for $25 \mathrm{sec}$

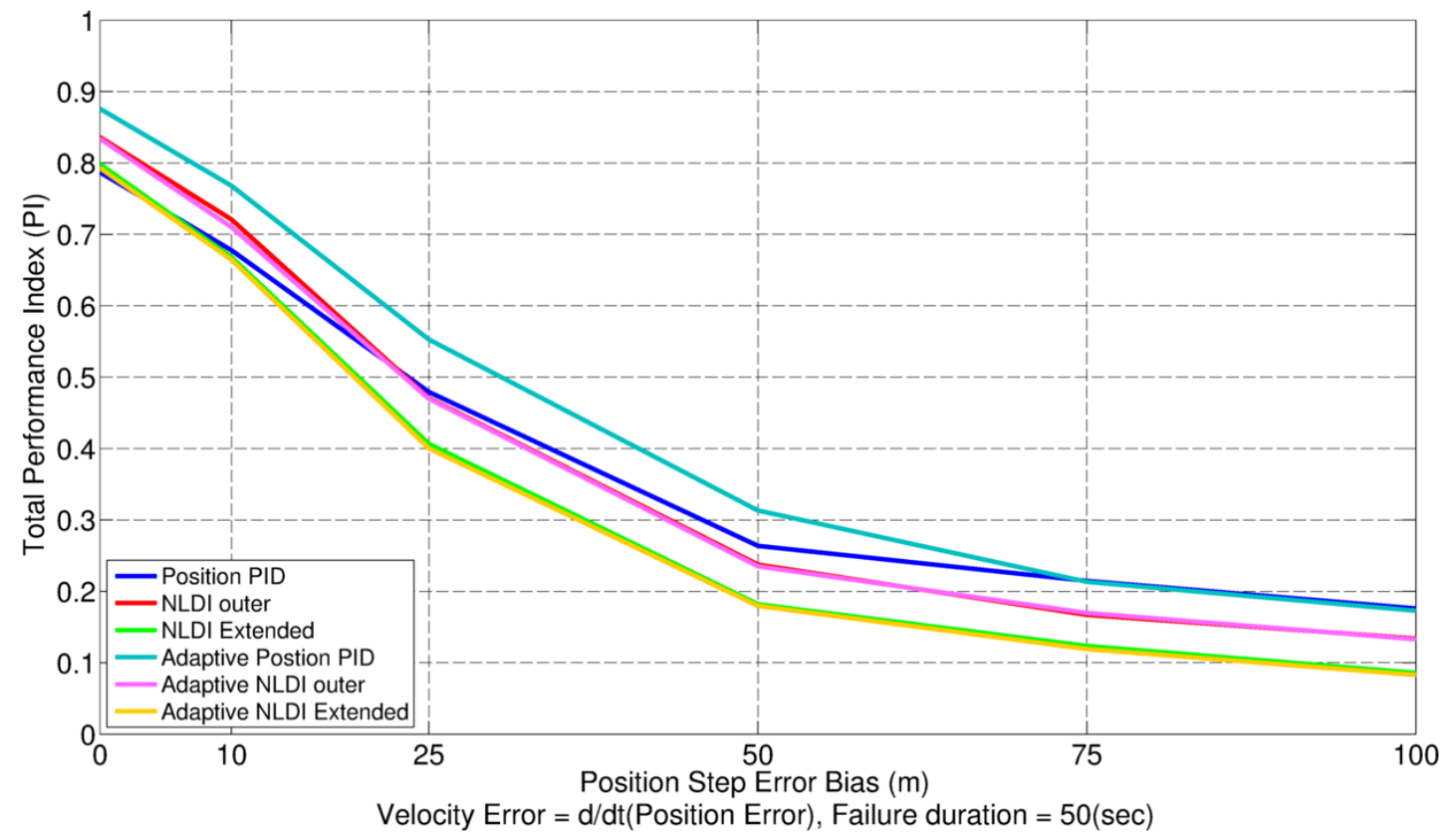

Figure 87: Controllers' Performance Index vs. Measurement Bias for $50 \mathrm{sec}$ 


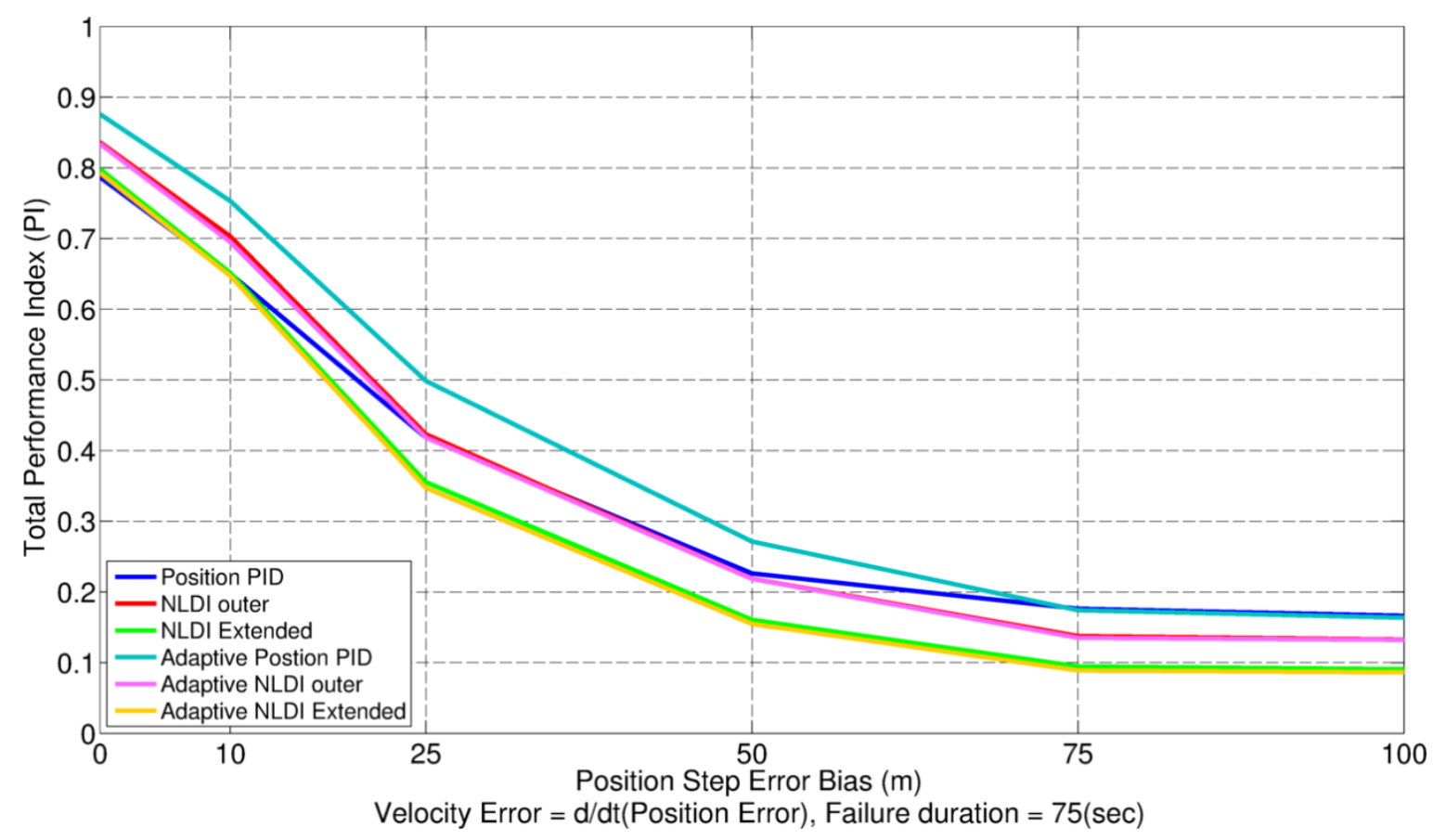

Figure 88: Controllers' Performance Index vs. Measurement Bias for $75 \mathrm{sec}$

Table 16 summarizes the step analysis results. For each controller, the table shows the maximum 3 dimensional error occurred during that test and the distance between the commanded end point and the aircraft actual end position. This distance is a measure of how well was the controller able to recover after the failure.

\begin{tabular}{|c|c|c|c|c|c|c|c|c|c|c|c|c|c|}
\hline & \multirow[b]{2}{*}{ Step Value } & \multicolumn{2}{|c|}{ Position PID } & \multicolumn{2}{|c|}{ NLDI Outer-loop } & \multicolumn{2}{|c|}{ NLDI Extended } & \multicolumn{2}{|c|}{ Adaptive PID } & \multicolumn{2}{|c|}{ Adaptive Outer } & \multicolumn{2}{|c|}{ Adaptive Extended } \\
\hline & & Max XYZ & End Error & Max XYZ & End Error & Max XYZ & End Error & Max XYZ & End Error & Max XYZ & End Error & Max XYZ & End Error \\
\hline \multirow{5}{*}{ Step for $12 \mathrm{sec}$} & 10 & 28.44 & 15.30 & 23.66 & 4.16 & 23.16 & 4.26 & 23.83 & 4.25 & 24.92 & 4.22 & 24.69 & 4.23 \\
\hline & 25 & 52.02 & 15.30 & 53.12 & 4.28 & 56.85 & 4.32 & 47.48 & 4.24 & 50.07 & 4.13 & 79.27 & 4.39 \\
\hline & 50 & 98.22 & 15.31 & 100.91 & 4.25 & 105.79 & 4.17 & 94.93 & 4.25 & 119.62 & 4.14 & 160.99 & 4.14 \\
\hline & 75 & 144.81 & 15.31 & 177.92 & 4.12 & 184.99 & 4.22 & 141.53 & 4.26 & 212.00 & 4.04 & 226.88 & 4.26 \\
\hline & 100 & 192.75 & 15.31 & 250.53 & 4.22 & 267.29 & 4.14 & 219.40 & 4.25 & 325.34 & 4.13 & 289.72 & 4.14 \\
\hline \multirow{5}{*}{ Step for $25 \mathrm{sec}$} & 10 & 33.83 & 15.31 & 31.74 & 4.22 & 33.42 & 4.31 & 27.96 & 4.25 & 31.11 & 4.12 & 38.04 & 4.42 \\
\hline & 25 & 74.30 & 15.31 & 74.12 & 4.15 & 78.62 & 4.15 & 71.16 & 4.24 & 71.48 & 4.13 & 103.17 & 3.99 \\
\hline & 50 & 145.46 & 15.30 & 144.04 & 4.19 & 145.43 & 4.21 & 142.38 & 4.25 & 162.00 & 4.08 & 171.55 & 4.18 \\
\hline & 75 & 219.14 & 15.31 & 216.23 & 4.23 & 217.23 & 4.28 & 215.18 & 4.24 & 271.64 & 4.16 & 228.58 & 4.22 \\
\hline & 100 & 318.46 & 15.30 & 341.55 & 4.27 & 299.22 & 4.28 & 371.23 & 4.25 & 404.81 & 4.19 & 307.56 & 4.41 \\
\hline \multirow{5}{*}{ Step for $50 \mathrm{sec}$} & 10 & 34.31 & 15.30 & 24.53 & 4.17 & 23.09 & 4.15 & 24.16 & 4.25 & 25.00 & 4.18 & 25.11 & 4.04 \\
\hline & 25 & 62.61 & 15.31 & 54.03 & 4.25 & 56.79 & 4.33 & 51.20 & 4.25 & 56.91 & 4.06 & 80.05 & 4.43 \\
\hline & 50 & 113.80 & 15.30 & 155.82 & 4.21 & 151.63 & 4.13 & 101.17 & 4.25 & 171.01 & 4.26 & 142.92 & 4.10 \\
\hline & 75 & 195.76 & 15.31 & 236.25 & 4.14 & 242.21 & 4.24 & 183.82 & 4.24 & 280.41 & 4.29 & 229.99 & 4.41 \\
\hline & 100 & 288.32 & 15.31 & 323.87 & 4.32 & 347.86 & 4.31 & 293.40 & 4.25 & 392.22 & 4.17 & 325.86 & 4.15 \\
\hline \multirow{5}{*}{ Step for $75 \mathrm{sec}$} & 10 & 34.73 & 15.32 & 24.57 & 4.18 & 23.07 & 4.13 & 23.44 & 4.25 & 25.14 & 4.13 & 24.81 & 4.11 \\
\hline & 25 & 62.94 & 15.29 & 53.75 & 4.23 & 57.04 & 4.11 & 51.21 & 4.24 & 56.83 & 4.23 & 80.21 & 4.15 \\
\hline & 50 & 112.06 & 15.30 & 149.31 & 4.18 & 142.00 & 4.16 & 101.20 & 4.25 & 173.75 & 4.22 & 142.30 & 4.11 \\
\hline & 75 & 186.59 & 15.29 & 212.77 & 4.19 & 234.39 & 4.21 & 175.92 & 4.25 & 274.07 & 4.15 & 230.88 & 4.35 \\
\hline & 100 & 276.56 & 15.30 & 239.94 & 4.23 & 314.79 & 4.20 & 286.89 & 4.24 & 352.77 & 4.26 & 302.47 & 4.17 \\
\hline
\end{tabular}

Table 16: Step Sensitivity Analysis Summary 
As it can be seen from the table, all the controllers suffer from a large position error increased with the step failure value increment; however, all the controllers restored the aircraft to actual path after the failure duration was over.

\subsubsection{Ramp Sensitivity Analysis}

Ramp is the most frequently occurring failure in the GPS failure mode table. The controllers' performance was evaluated against a position ramp slope of $0.6,1.5,2.5,5$ and 10 meter per second for $12,25,50$, and 75 seconds.

The effect of the ramp failures on the controllers was similar to the step; all the controllers followed the ramp error thus the same effect can be seen on their trajectory tracking indices. The results for a failure duration of 12 seconds are presented in Figure 89, Figure 90, and Figure 91.

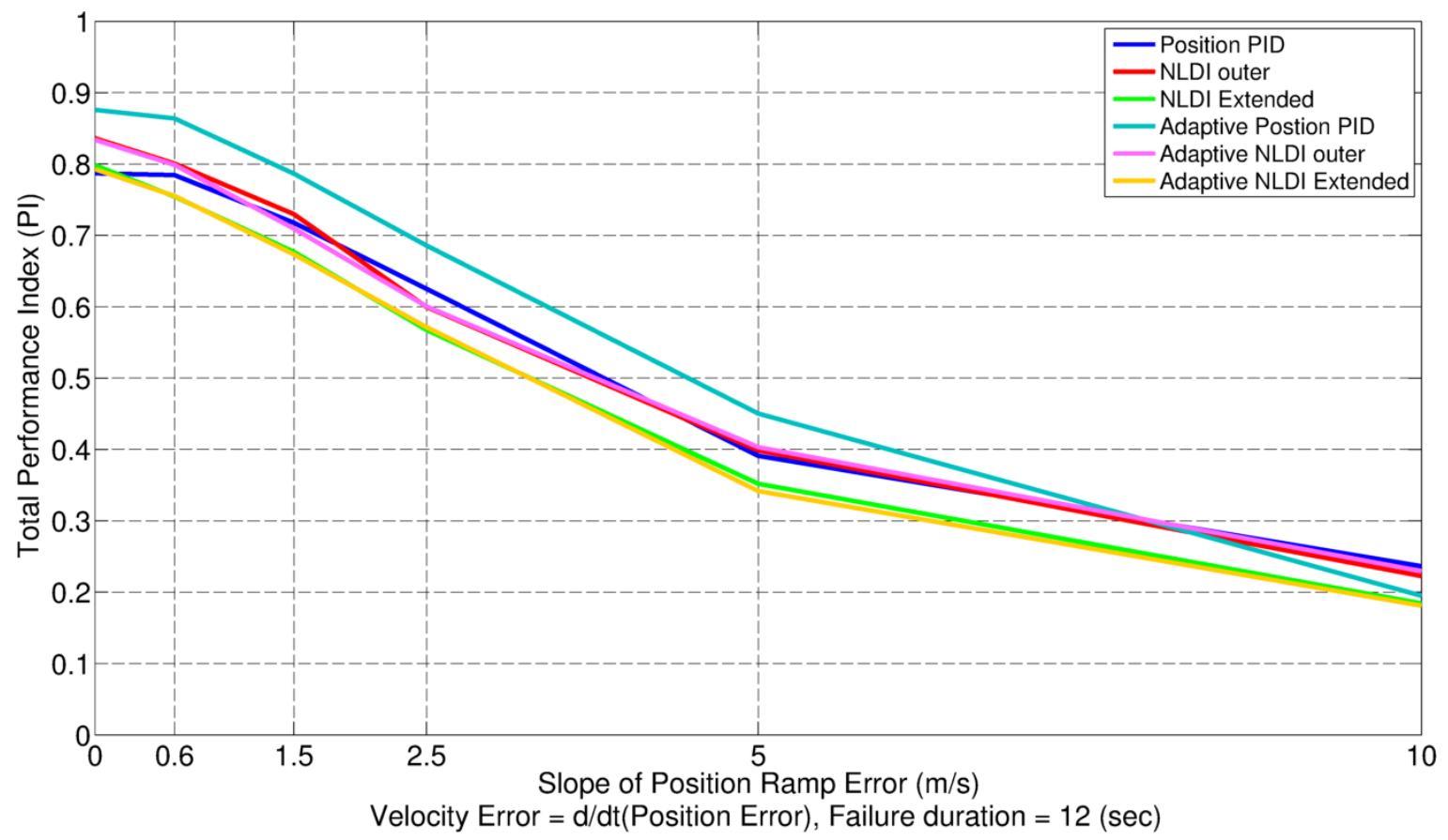

Figure 89: Controllers' Performance Index vs. Ramp Measurement Error for $12 \mathrm{sec}$ 


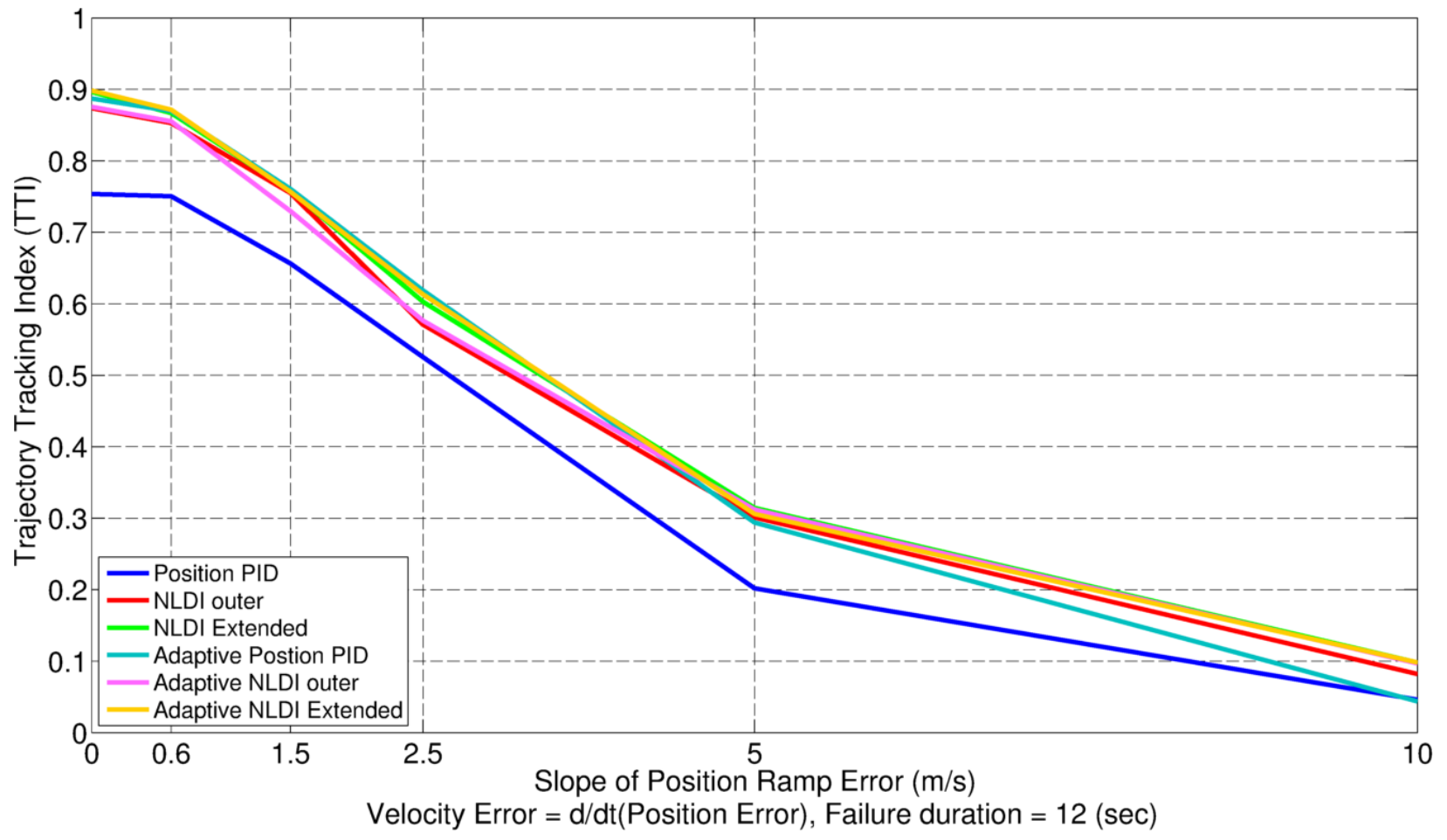

Figure 90: Controllers' CAI vs. Ramp Measurement Error for $12 \mathrm{sec}$

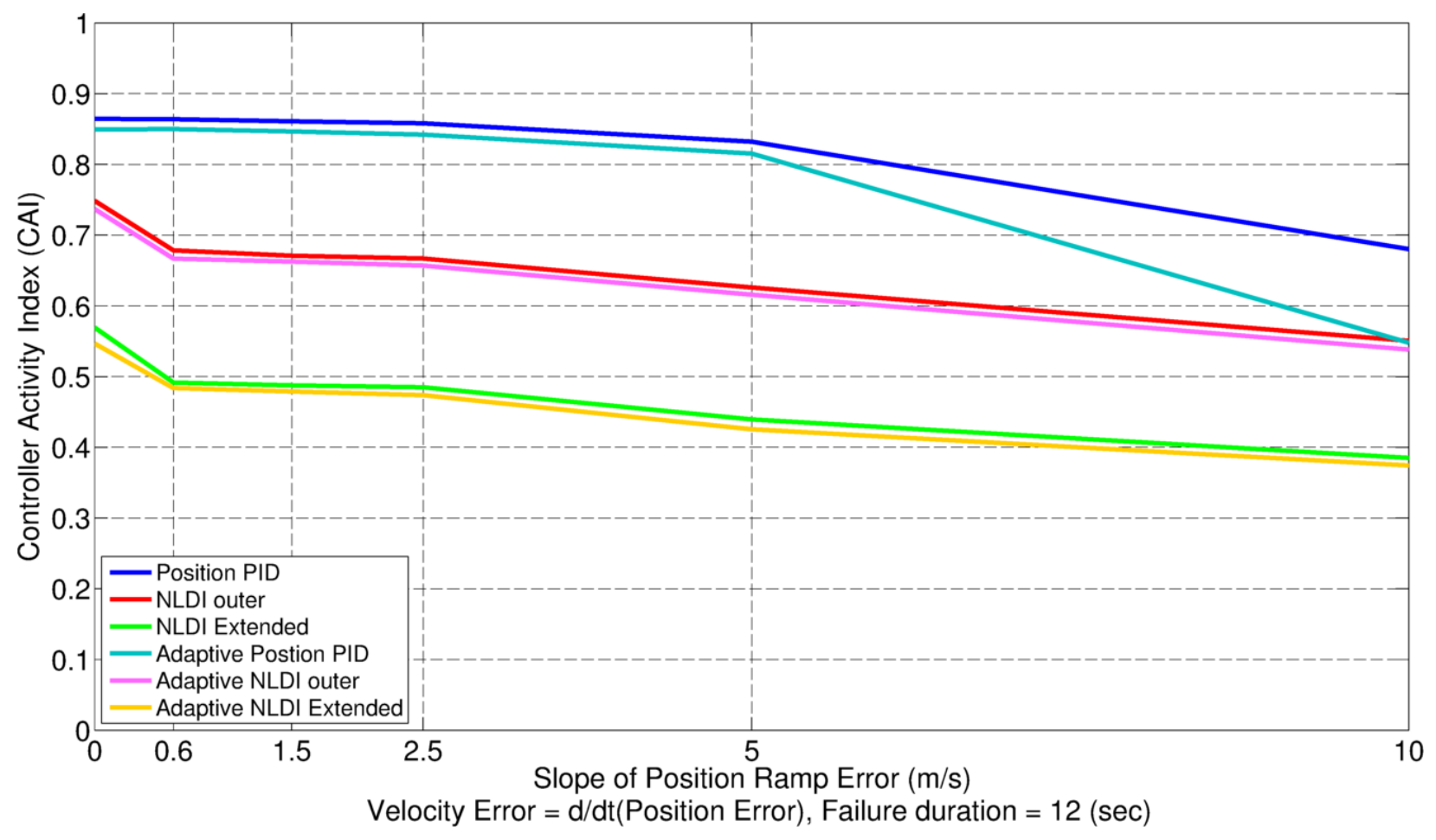

Figure 91: Controllers' CAl vs. Ramp Measurement Error for $12 \mathrm{sec}$

The effect of the ramp failures on the controllers was similar to the step, all the controllers followed the ramp error thus the same effect can be seen on their trajectory tracking indices. In 
general, the TTI drops abruptly with the increase of the ramp slope, while the impact on CAI is less significant. The most robust controller with respect to this parameter is the adaptive PID. Figure 92, Figure 93, and Figure 94 present the 25, 50, and 75 second ramp cases.

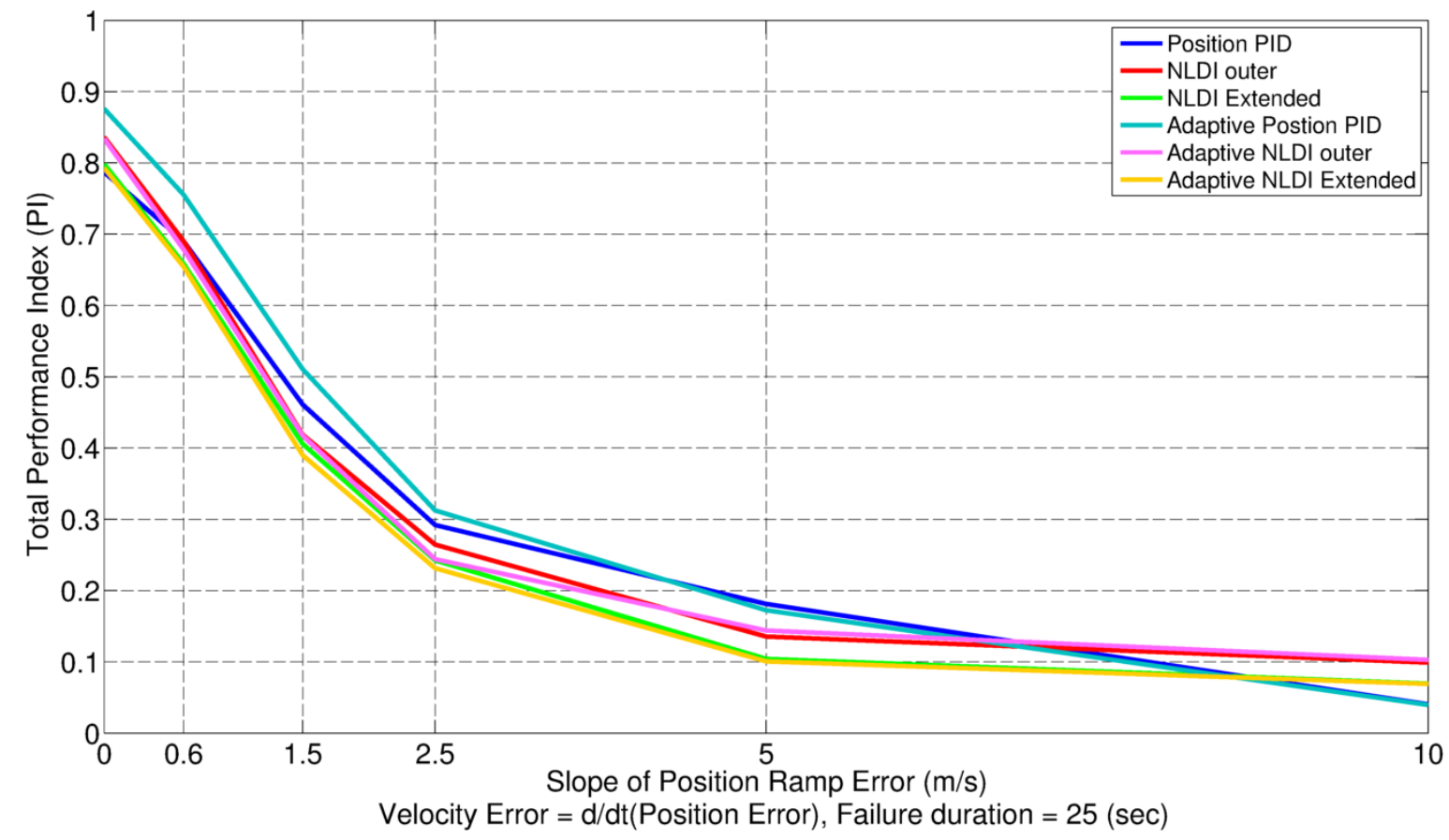

Figure 92: Controllers' Performance Index vs. Ramp Measurement Error for $25 \mathrm{sec}$

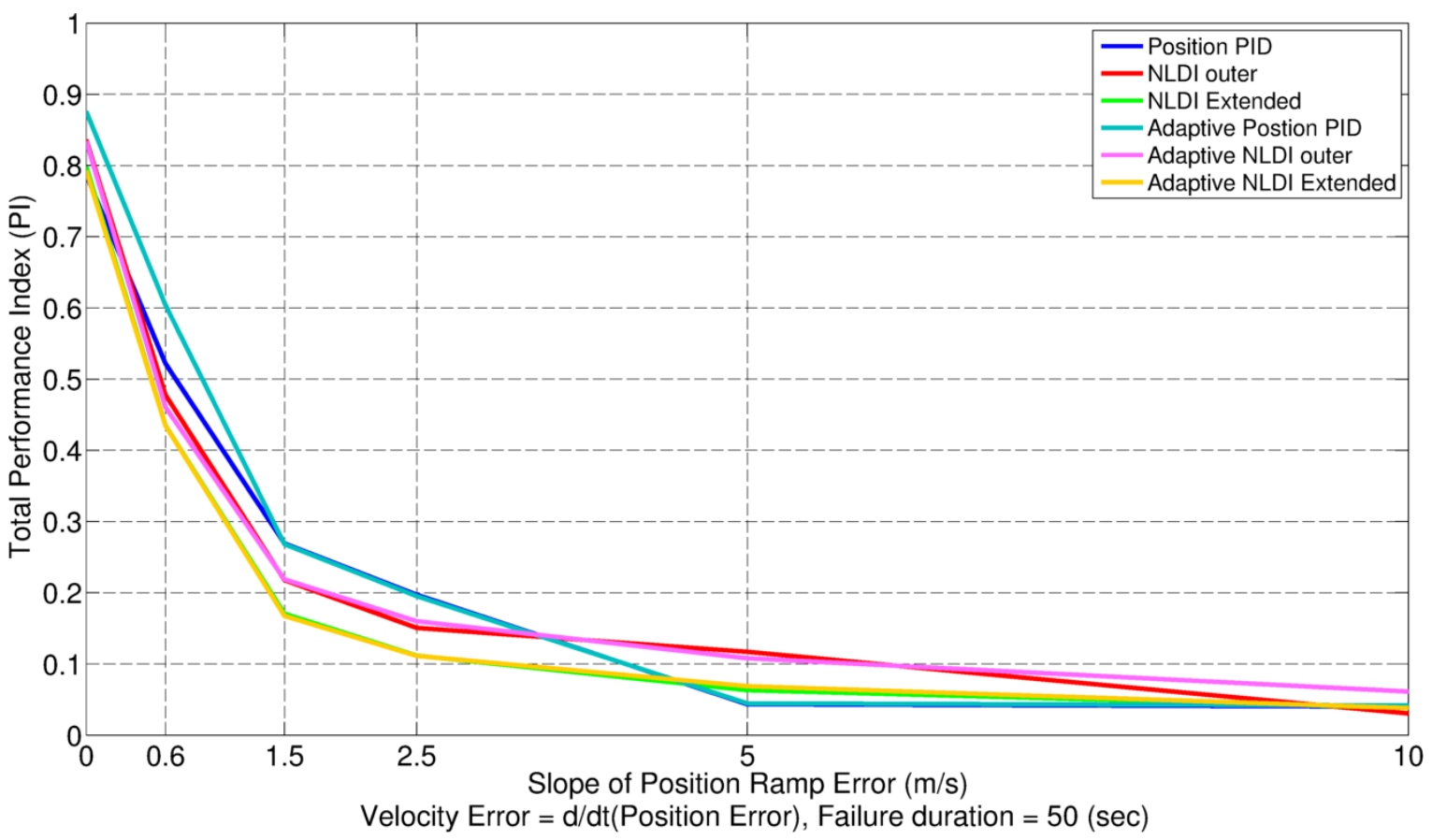

Figure 93: Controllers' Performance Index vs. Ramp Measurement Error for $50 \mathrm{sec}$ 


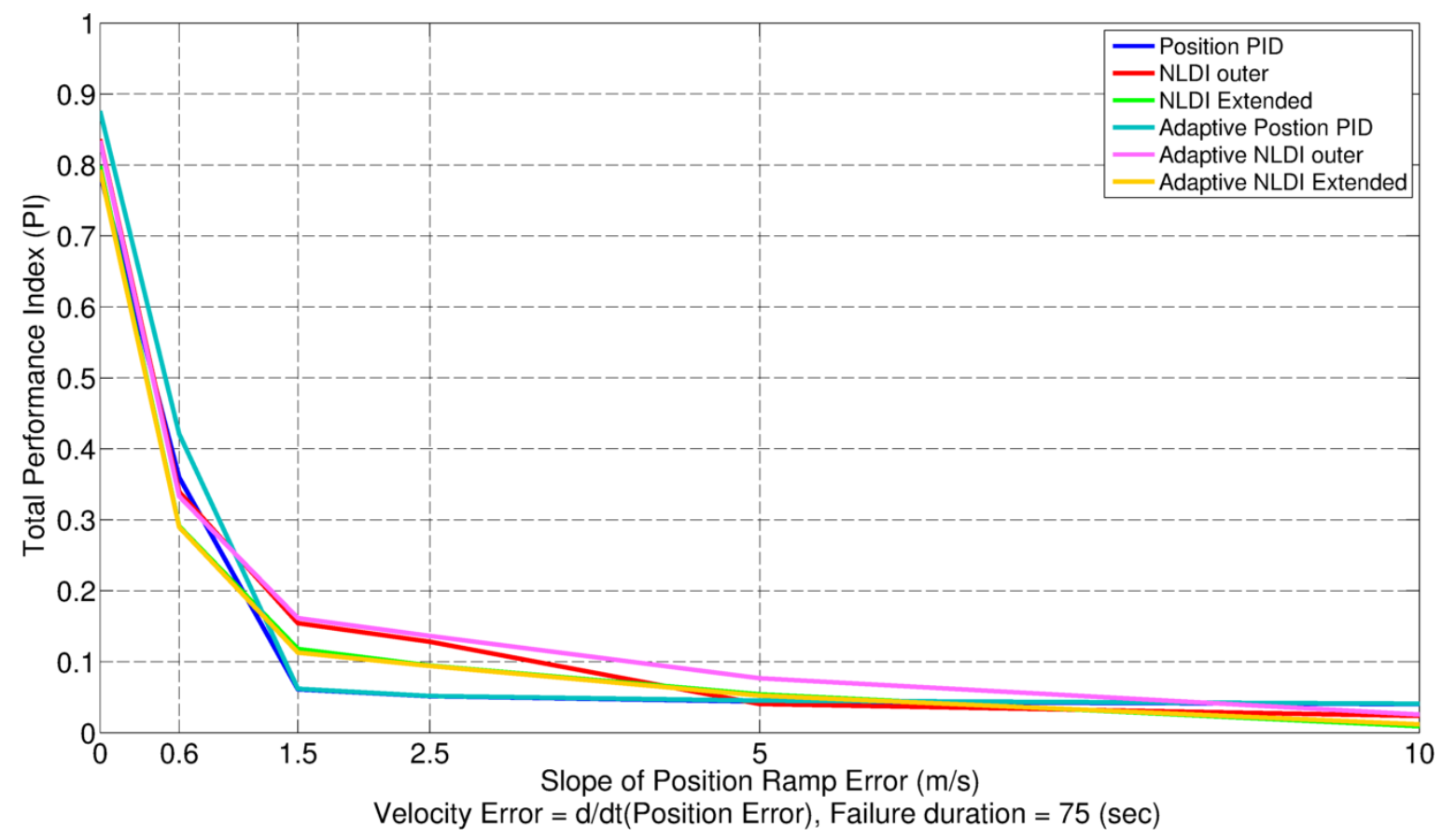

Figure 94: Controllers' Performance Index vs. Ramp Measurement Error for 75 sec

Table 17 summarizes the ramp failure effect. As expected, larger tracking errors are recorded for longer failure duration. It can be noticed that the adaptive NLDI extended controllers overcome the failure and manage to fly the aircraft to the destination point. In general, the position PID controllers were the most robust with respect to this type of failure.

\begin{tabular}{|c|c|c|c|c|c|c|c|c|c|c|c|c|c|}
\hline & \multirow[b]{2}{*}{ Ranp Slope } & \multicolumn{2}{|c|}{ Position PID } & \multicolumn{2}{|c|}{ NLDI Outer-loop } & \multicolumn{2}{|c|}{ NLDI Extended } & \multicolumn{2}{|c|}{ Adaptive PID } & \multicolumn{2}{|c|}{ Adaptive Outer } & \multicolumn{2}{|c|}{ Adaptive Extended } \\
\hline & & $\operatorname{Max}$ XYZ & End Error & Max XYZ & End Error & Max XYZ & End Error & MAXXYZ & End Error & Max XYZ & End Error & $\operatorname{Max} \mathrm{XYZ}$ & End Error \\
\hline \multirow{5}{*}{ Ramp for $12 \mathrm{sec}$} & 0.6 & 19.95 & 15.30 & 21.80 & 4.29 & 18.18 & 4.09 & 18.70 & 4.25 & 21.71 & 4.10 & 16.81 & 4.12 \\
\hline & 1.5 & 37.07 & 15.31 & 34.59 & 4.13 & 36.11 & 4.15 & 36.36 & 4.24 & 36.34 & 4.22 & 36.17 & 4.00 \\
\hline & 2.5 & 59.94 & 15.31 & 58.96 & 4.27 & 59.47 & 4.08 & 60.15 & 4.25 & 60.12 & 4.07 & 59.52 & 4.10 \\
\hline & 5 & 121.63 & 15.30 & 123.51 & 4.26 & 119.33 & 4.14 & 121.08 & 4.24 & 136.83 & 4.25 & 119.83 & 4.28 \\
\hline & 10 & 253.97 & 15.28 & 252.33 & 4.18 & 240.69 & 4.15 & 588.47 & 4.26 & 339.10 & 4.13 & 239.24 & 4.10 \\
\hline \multirow{5}{*}{ Ramp for $25 \mathrm{sec}$} & 0.6 & 42.38 & 15.31 & 39.69 & 4.19 & 39.76 & 4.11 & 40.10 & 4.25 & 39.96 & 4.10 & 39.70 & 4.05 \\
\hline & 1.5 & 100.25 & 15.31 & 101.27 & 4.26 & 98.79 & 4.32 & 99.85 & 4.25 & 116.72 & 4.10 & 98.76 & 4.18 \\
\hline & 2.5 & 166.37 & 15.31 & 173.33 & 4.16 & 165.12 & 4.11 & 166.72 & 4.24 & 228.90 & 4.09 & 177.29 & 4.09 \\
\hline & 5 & 440.79 & 15.30 & 425.64 & 4.22 & 467.78 & 4.12 & 549.29 & 4.25 & 574.72 & 4.11 & 441.07 & 4.06 \\
\hline & 10 & 3508.60 & 2647.50 & 787.07 & 4.26 & 1269.50 & 4.31 & 3026.10 & 2168.40 & 1248.70 & 4.12 & 1027.60 & 4.07 \\
\hline \multirow{5}{*}{ Ramp for $50 \mathrm{sec}$} & 0.6 & 68.43 & 15.30 & 91.74 & 4.26 & 87.61 & 4.28 & 53.21 & 4.24 & 99.34 & 4.14 & 85.23 & 4.04 \\
\hline & 1.5 & 199.07 & 15.31 & 256.05 & 4.30 & 251.13 & 4.18 & 185.85 & 4.25 & 300.43 & 4.10 & 248.61 & 4.03 \\
\hline & 2.5 & 388.09 & 15.30 & 445.27 & 4.29 & 476.16 & 4.05 & 418.11 & 4.25 & 556.43 & 4.21 & 453.96 & 4.20 \\
\hline & 5 & 2650.80 & 2650.70 & 672.75 & 4.34 & 981.14 & 4.12 & 2438.50 & 2438.50 & 952.22 & 4.21 & 883.30 & 4.06 \\
\hline & 10 & 2790.40 & 2589.50 & 1029.80 & 292.77 & 1347.20 & 4.25 & 2681.50 & 2681.50 & 1265.60 & 4.47 & 1241.90 & 4.40 \\
\hline \multirow{5}{*}{ Ramp for $75 \mathrm{sec}$} & 0.6 & 98.41 & 15.30 & 140.67 & 4.19 & 137.05 & 4.06 & 81.83 & 4.26 & 155.71 & 4.13 & 136.07 & 4.06 \\
\hline & 1.5 & 3885.10 & 3885.10 & 259.49 & 4.29 & 349.02 & 4.14 & 2960.30 & 2554.20 & 378.70 & 4.13 & 338.36 & 4.09 \\
\hline & 2.5 & 2639.10 & 2639.10 & 386.48 & 4.25 & 457.13 & 4.31 & 2442.80 & 2400.40 & 472.38 & 4.31 & 451.19 & 4.26 \\
\hline & 5 & 2813.60 & 2813.60 & 751.74 & 11.21 & 801.35 & 4.27 & 2557.00 & 2557.00 & 802.68 & 4.12 & 803.51 & 4.32 \\
\hline & 10 & 2944.60 & 2851.00 & 1478.50 & 1018.70 & 1522.80 & 672.48 & 2840.60 & 2840.60 & 1512.30 & 624.81 & 1510.70 & 404.30 \\
\hline
\end{tabular}

Table 17: Ramp Sensitivity Analysis Summary 


\subsubsection{Sinusoidal Sensitivity Analysis}

The sinusoidal error is listed in the GPS SPS performance standard document as one of GPS SPS SIS integrity failures; however, no information was found about the expected value or frequency of this failure. The magnitude of the sinusoidal failure in the following analysis was assumed to be the same as the magnitude of the step failure, and the duration of this failure was assumed to be 35 seconds. The sinusoidal position and velocity errors affect the controllers' performance in a similar way as the step and ramp failures. All the controllers experienced large trajectory tracking errors without significant change in their control activity indices. Figure 95 illustrates the effect of the sinusoidal failure on the controllers' performances. Furthermore, Figure 96 and Figure 97 present the controllers' TTI and CAI respectively. Figures 94, 95, and 96 present the variation of the PI with amplitude of the sinusoidal error for durations of the failure of 25,50 , and 75 seconds, respectively. Similar trends are recorded as in the case of the 12-second failure; however, the performance degradation is more abrupt as the duration of the failure increases, as expected.

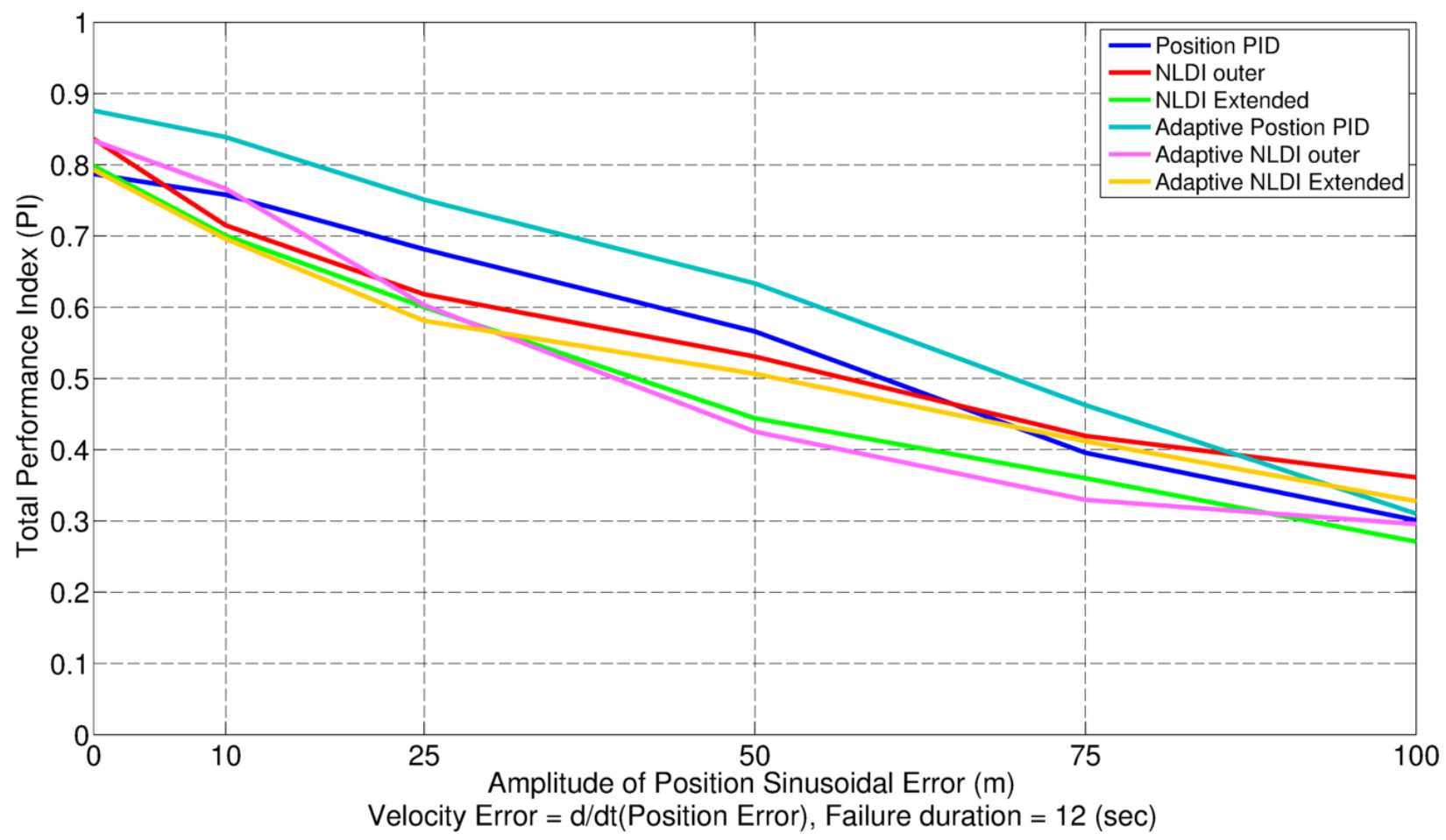

Figure 95: Controllers Performance Index vs. Sinusoidal Measurement Error for $12 \mathrm{sec}$ 


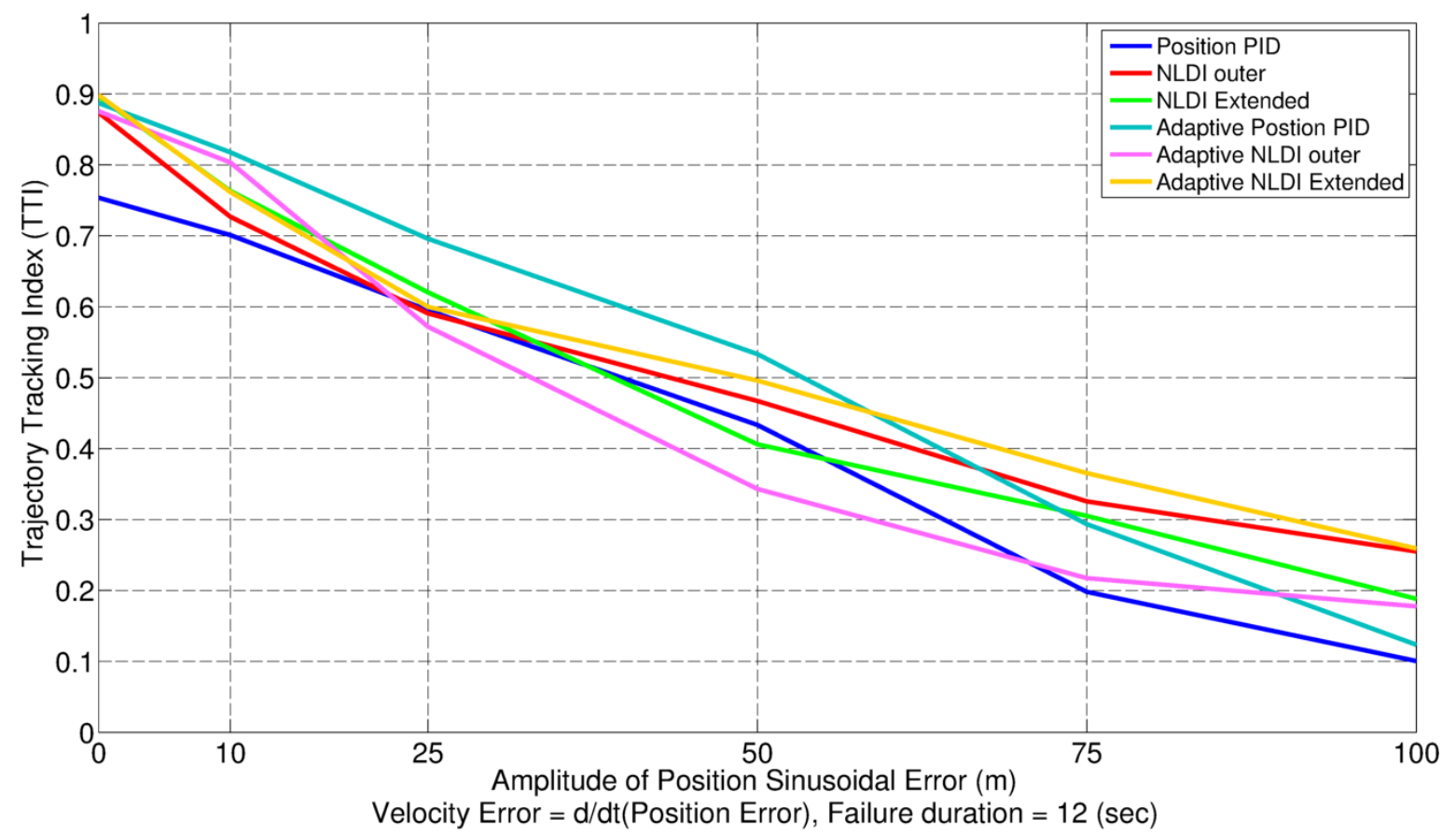

Figure 96: Controllers TTI vs. Sinusoidal Measurement Error for $12 \mathrm{sec}$

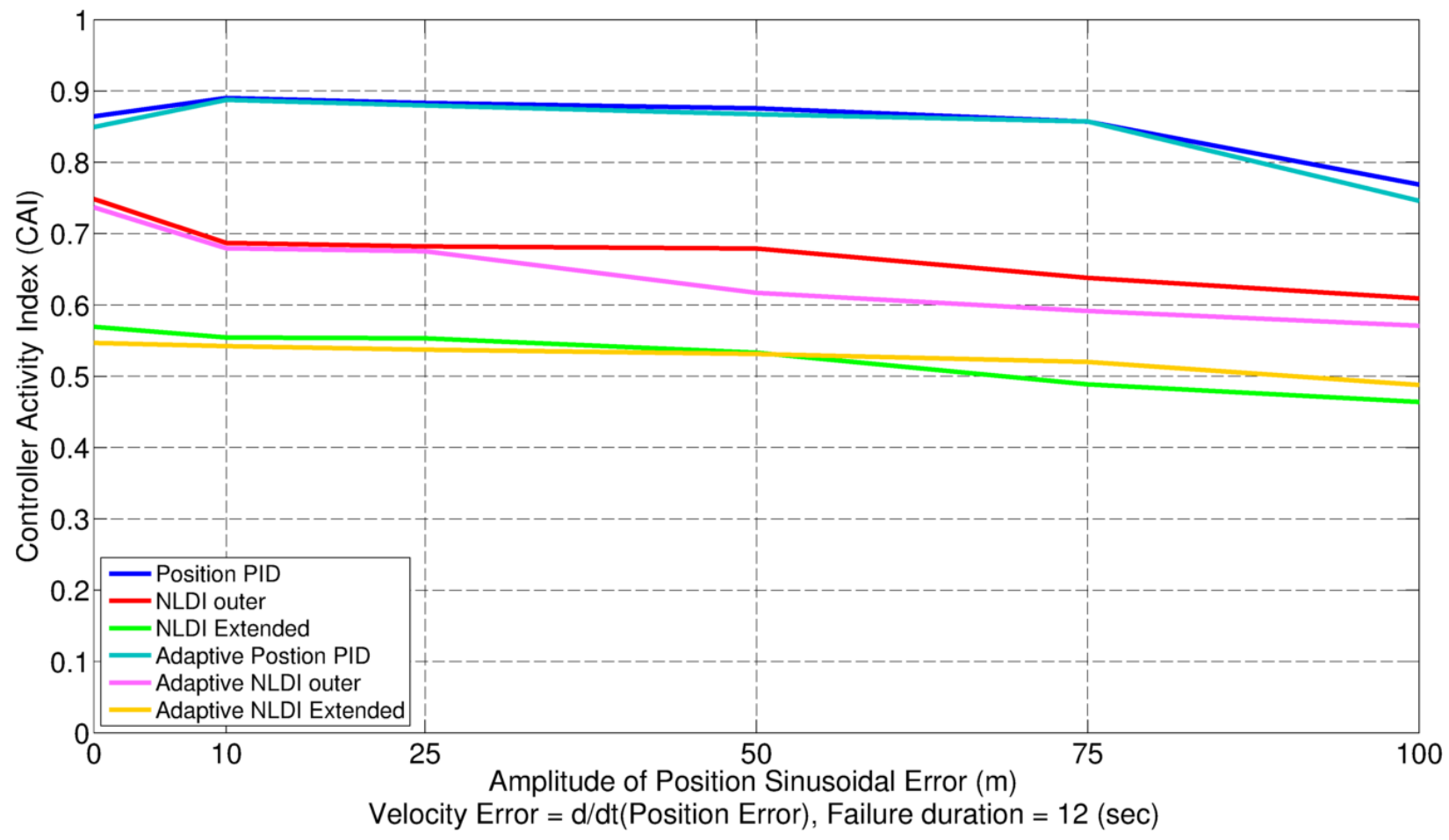

Figure 97: Controllers CAI vs. Sinusoidal Measurement Error for $12 \mathrm{sec}$ 


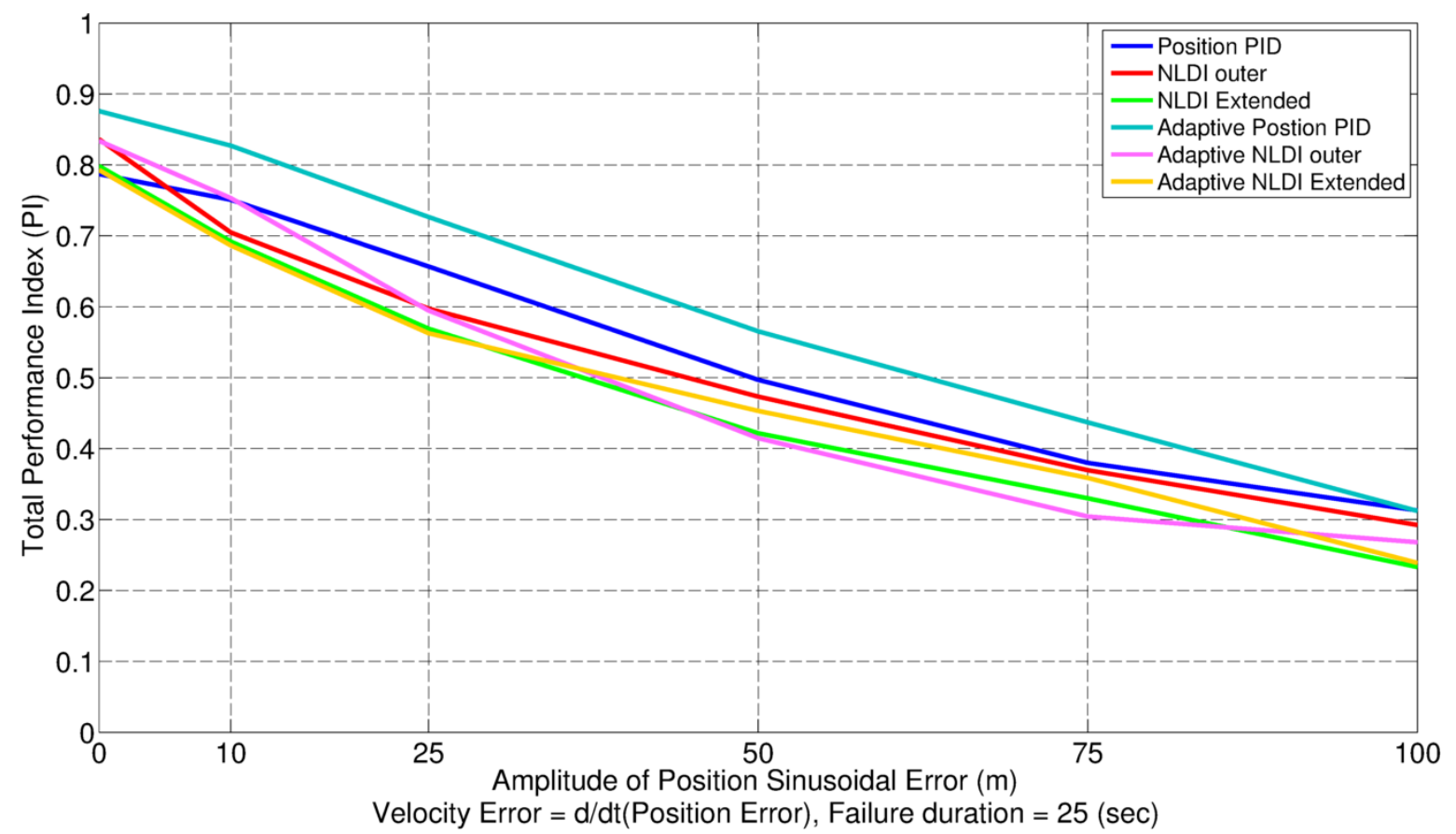

Figure 98: Controllers TTI vs. Sinusoidal Measurement Error for $25 \mathrm{sec}$

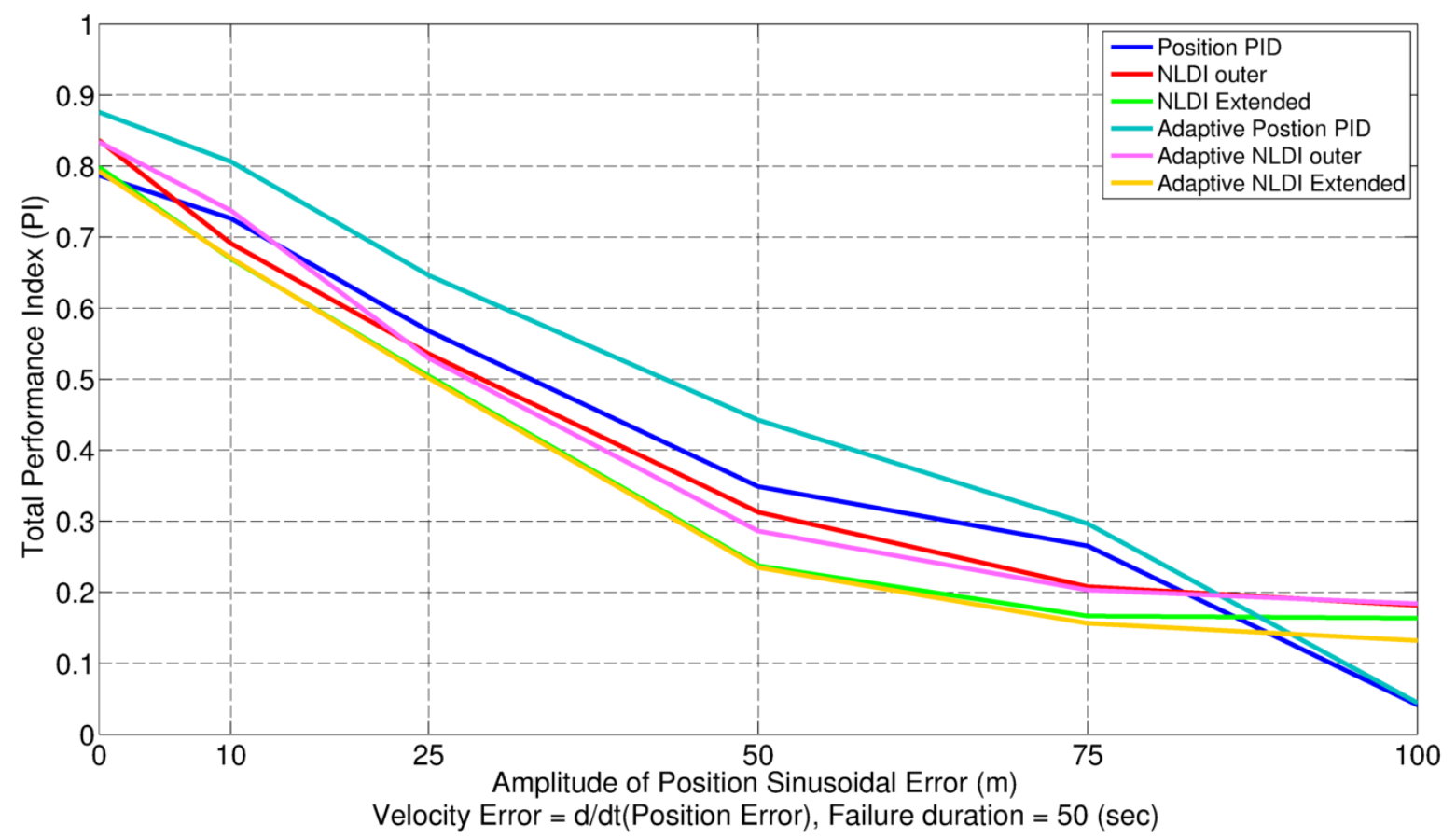

Figure 99: Controllers TTI vs. Sinusoidal Measurement Error for $50 \mathrm{sec}$ 


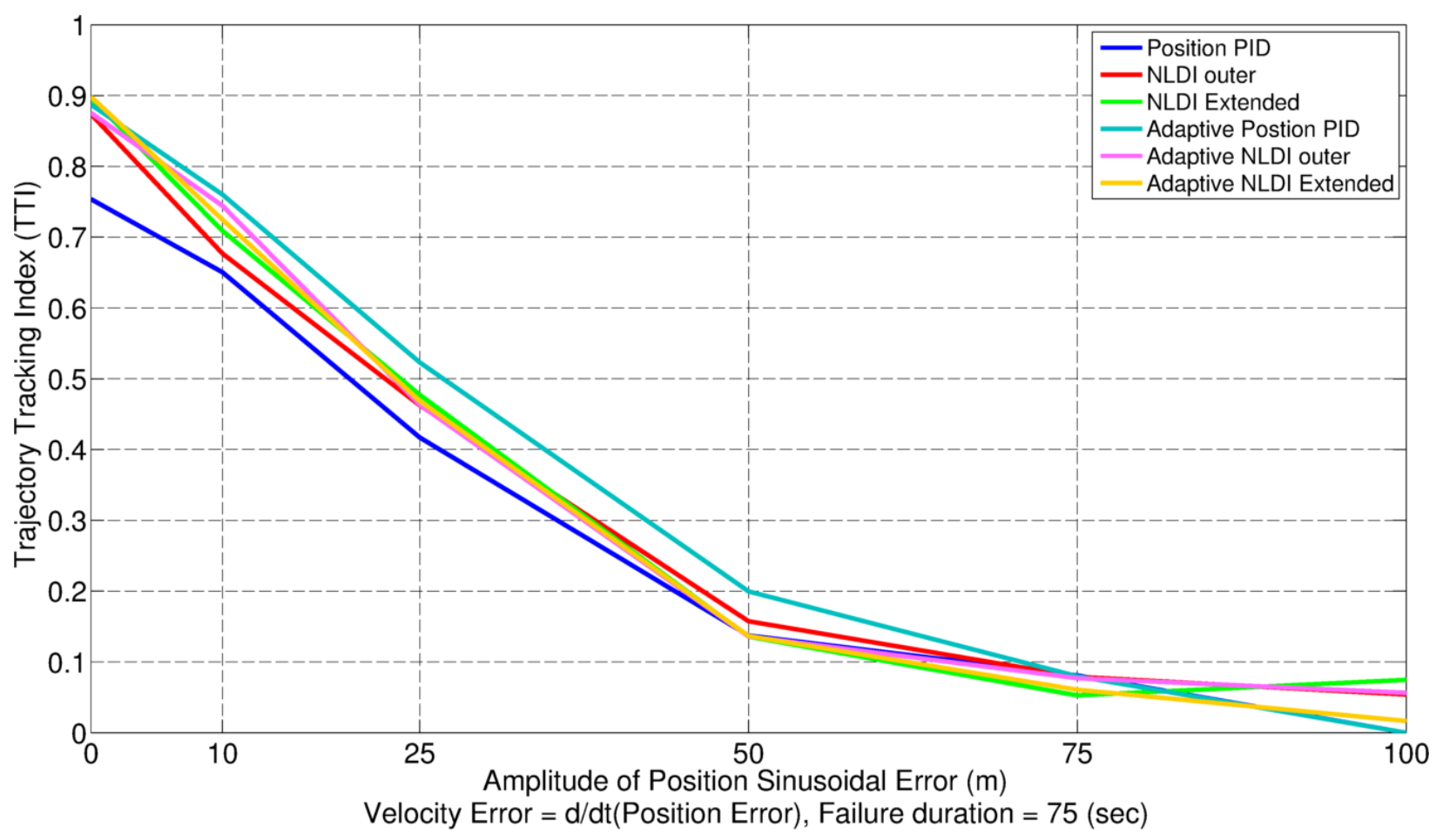

Figure 100: Controllers TTI vs. Sinusoidal Measurement Error for $75 \mathrm{sec}$

Finally, Table 18 summarizes the sinusoidal failure analysis. Large trajectory tracking errors are noticed, as expected, with most of the controllers recovering the aircraft back to the commanded path after failure. The only exception is the effect of long duration large amplitude sinusoidal failure on the position PID controllers.

\begin{tabular}{|c|c|c|c|c|c|c|c|c|c|c|c|c|c|}
\hline & \multirow[b]{2}{*}{ Sine Amplitude } & \multicolumn{2}{|c|}{ Position PID } & \multicolumn{2}{|c|}{ NLDI Outer-loop } & \multicolumn{2}{|c|}{ NLDI Extended } & \multicolumn{2}{|c|}{ Adaptive PID } & \multicolumn{2}{|c|}{ Adaptive Outer } & \multicolumn{2}{|c|}{ Adaptive Extended } \\
\hline & & Max XYZ & End Error & Max XYZ & End Error & Max XYZ & End Error & MAX XYZ & End Error & Max XYZ & End Error & Max XYZ & End Error \\
\hline \multirow{5}{*}{ Sine for $12 \mathrm{sec}$} & 10 & 31.78 & 15.09 & 51.95 & 4.61 & 43.34 & 4.48 & 29.70 & 4.58 & 30.74 & 4.53 & 44.06 & 4.32 \\
\hline & 25 & 46.52 & 15.08 & 93.66 & 4.63 & 89.83 & 4.64 & 46.73 & 4.57 & 93.84 & 4.53 & 102.04 & 4.42 \\
\hline & 50 & 75.59 & 15.08 & 84.20 & 4.54 & 135.39 & 4.50 & 77.65 & 4.57 & 159.51 & 4.57 & 94.06 & 4.39 \\
\hline & 75 & 130.11 & 15.08 & 129.22 & 4.60 & 162.02 & 4.54 & 126.97 & 4.57 & 229.80 & 4.54 & 104.64 & 4.46 \\
\hline & 100 & 195.05 & 15.08 & 166.23 & 4.69 & 222.64 & 4.72 & 197.60 & 4.56 & 320.04 & 4.49 & 148.85 & 4.51 \\
\hline \multirow{5}{*}{ Sine for $25 \mathrm{sec}$} & 10 & 31.85 & 15.08 & 51.90 & 4.66 & 43.42 & 4.57 & 30.31 & 4.57 & 31.29 & 4.52 & 44.17 & 4.45 \\
\hline & 25 & 48.14 & 15.08 & 94.42 & 4.56 & 92.73 & 4.52 & 45.79 & 4.56 & 94.48 & 4.60 & 97.15 & 4.40 \\
\hline & 50 & 92.49 & 15.08 & 93.66 & 4.65 & 135.05 & 4.49 & 82.43 & 4.57 & 161.56 & 4.52 & 93.08 & 4.51 \\
\hline & 75 & 193.83 & 15.08 & 202.47 & 4.64 & 176.60 & 4.60 & 200.07 & 4.57 & 272.23 & 4.52 & 154.55 & 4.59 \\
\hline & 100 & 262.03 & 15.08 & 265.75 & 4.55 & 478.50 & 4.68 & 291.27 & 4.57 & 497.43 & 4.59 & 334.89 & 4.50 \\
\hline \multirow{5}{*}{ Sine for $50 \mathrm{sec}$} & 10 & 31.94 & 15.08 & 51.97 & 4.61 & 45.82 & 4.65 & 30.28 & 4.57 & 31.07 & 4.53 & 44.57 & 4.43 \\
\hline & 25 & 56.35 & 15.09 & 94.02 & 4.64 & 92.35 & 4.69 & 48.33 & 4.57 & 93.08 & 4.58 & 95.25 & 4.70 \\
\hline & 50 & 128.27 & 15.08 & 142.71 & 4.53 & 202.55 & 4.59 & 117.49 & 4.58 & 163.45 & 4.56 & 226.70 & 4.46 \\
\hline & 75 & 238.44 & 15.08 & 253.31 & 4.56 & 326.43 & 4.54 & 232.82 & 4.58 & 296.16 & 4.53 & 381.92 & 4.68 \\
\hline & 100 & 3095.00 & 1750.10 & 372.25 & 4.65 & 697.49 & 4.47 & 3258.40 & 1449.20 & 509.85 & 4.51 & 362.33 & 4.64 \\
\hline \multirow{5}{*}{ Sine for $75 \mathrm{sec}$} & 10 & 32.80 & 15.08 & 52.06 & 4.58 & 45.00 & 4.49 & 30.42 & 4.57 & 31.44 & 4.54 & 40.55 & 4.47 \\
\hline & 25 & 56.42 & 15.09 & 93.55 & 4.57 & 87.73 & 4.71 & 48.32 & 4.58 & 94.33 & 4.51 & 94.74 & 4.71 \\
\hline & 50 & 157.89 & 15.08 & 116.31 & 4.59 & 183.41 & 4.73 & 123.57 & 4.58 & 163.95 & 4.62 & 191.59 & 4.44 \\
\hline & 75 & 271.77 & 15.08 & 270.82 & 4.54 & 389.79 & 4.56 & 258.61 & 4.57 & 295.28 & 4.51 & 416.11 & 4.55 \\
\hline & 100 & 3095.00 & 1748.50 & 374.58 & 4.59 & 699.62 & 4.53 & 3261.00 & 1434.90 & 545.66 & 4.53 & 548.68 & 4.54 \\
\hline
\end{tabular}

Table 18: Sinusoidal Sensitivity Analysis Summary 


\subsection{Analysis Summary}

Table 19 summarizes the "good", "adequate", and "poor" controllers' performances can be archived for GPS error model parameter using the best and the worst controller in that category.

\begin{tabular}{|l|c|c|c|c|c|c|}
\hline \multicolumn{1}{|c|}{$\begin{array}{c}\text { GPS Error model } \\
\text { Parameter }\end{array}$} & \multicolumn{2}{|c|}{ Range for a “good" PI } & \multicolumn{2}{c|}{$\begin{array}{c}\text { Range for an } \\
\text { "adequate" PI }\end{array}$} & \multicolumn{2}{c|}{$\begin{array}{c}\text { Range for a “crash" or } \\
\text { "lost" PI }\end{array}$} \\
\hline & Best & Worst & Best & Worst & Best & Worst \\
\hline Time Delay (sec) & $0-0.72$ & $0-0.2$ & $0.72-0.98$ & $0.2-0.56$ & N/A & $0.56-0.98$ \\
\hline $\begin{array}{l}\text { Position and velocity } \\
\text { update interval (sec) }\end{array}$ & $0-0.62$ & $0-0.14$ & $0.62-0.98$ & $0.14-0.40$ & N/A & $0.40-0.98$ \\
\hline $\begin{array}{l}\text { Velocity update } \\
\text { interval (sec) }\end{array}$ & $0-0.98$ & $0-0.22$ & N/A & $0.22-0.98$ & N/A & N/A \\
\hline $\begin{array}{l}\text { Error STD analysis (m) } \\
\text { (STD of velocity error } \\
\text { 0.65 STD position } \\
\text { error) }\end{array}$ & $0-1.5$ & $0-0.25$ & $1.5-4$ & $0.25-1.5$ & N/A & $1.5-4$ \\
\hline
\end{tabular}

Table 19: Ranges for Good, Adequate, and Poor Performances

For example, a performance value of 0.7 or better can be achieved in the presence of up to 0.72 seconds of time delay using the best controller in the time delay analysis, while a performance of 0.7 or better can be archived in the range of 0 to 0.2 seconds using the worst performing controller in the time delay case. 


\section{Conclusions and Future Work}

This thesis presented the development of a GPS error model in Matlab Simulink and its use to perform a sensitivity analysis of fault tolerant control laws with respect to GPS error parameters. A set of performance metrics for the evaluation of autonomous flight control laws algorithms was formulated in terms of tracking errors and control activity. The composition of various metrics within the performance index was performed using fixed and variable weights depending on the local characteristics of the commanded trajectory.

The alteration of GPS parameters has a significant effect on the performance of trajectory tracking control laws considered in this study, typically exhibiting low thresholds whose exceeding may lead to loss of control.

Ranges for the GPS parameters have been identified that can ensure specific levels of control performance under the limitations of this study. These ranges can be used for the selection of a suitable GPS receiver within the design of autonomous flight control laws.

The most significant effect on the control performance is produced by time delay and compound position and velocity failures. The control laws considered in this study exhibit the highest robustness with respect to velocity update rate and position and velocity error noise.

More complex control architectures designed to accommodate for actuator failures show improved robustness with respect to GPS abnormal conditions only in a limited number of cases. Considering that these control laws had been designed for fault tolerance with respect to actuator failures, it may be concluded that in order to ensure adequate robustness with respect to GPS parameter variation, customized design techniques must be developed specifically for that purpose.

The design of the performance evaluation index must be carefully approached such that it captures the specifics of each mission. The use within this study of variable weights in the composition of the total performance index is one valuable tool to ensure the level of versatility needed for performance metrics design. Additional mechanism must be considered in the future to improve the evaluation metrics. 
The proposed GPS model, as demonstrated in this thesis, has proved to be a valuable, flexible tool for testing and evaluation of the fault tolerant capabilities of autonomous flight control laws.

Future work must address some of the limitations of this study, such as:

- consideration of different aircraft and analysis of aircraft-specific effects;

- consideration of a larger diversity of paths and investigation of the effects of the failure occurrence moment along the path;

- consideration of a full experimental matrix and techniques to reduce it;

- identification and investigation of factors that cause high robustness variability of different control laws with respect to GPS parameter;

- formulation of quantitative criteria for GPS receiver selection. 


\section{Bibliography}

[1] M. Fudge, T. Stagliano, and S. Tsiao, "Non-traditional flight safety systems \& integrated vehicle health management systems," ITT Industries, Advanced Engineering and Sciences Division, vol. 2560, 2003.

[2] E. P. Anderson, R. W. Beard, and T. W. McLain, "Real-time dynamic trajectory smoothing for unmanned air vehicles," Control Systems Technology, IEEE Transactions on, vol. 13, pp. 471-477, 2005.

[3] J. Wang, M. Garratt, A. Lambert, J. J. Wang, S. Han, and D. Sinclair, "Integration of GPS/INS/vision sensors to navigate unmanned aerial vehicles," IAPRSSIS, vol. 37, pp. 963-9, 2008.

[4] J. Leyssens, "GNSS positioning for UAV Applications," ed: httpwwwgnssus, 2009.

[5] E. D. Kaplan and C. J. Hegarty, Understanding GPS: principles and applications: Artech house, 2005.

[6] G. S. P. Standard, "Retrieved on July 14, 2009," ed, 2008.

[7] (2012). William J. Hughes Technical Center WAAS Test Team. Available: http://www.nstb.tc.faa.gov/

[8] U. Government. (02/05/2013). Official U.S. Government information about the

GPS. Available: http://www.gps.gov/

[9] J. Rankin, "An error model for sensor simulation GPS and differential GPS," in Position Location and Navigation Symposium, 1994., IEEE, 1994, pp. 260-266.

[10] K. A. Redmill, J. I. Martin, U. Ozguner, and K. Tamura, "Sensor and data fusion design and evaluation with a virtual environment simulator," in Intelligent Vehicles Symposium, 2000. IV 2000. Proceedings of the IEEE, 2000, pp. 668-674.

[11] M. Perhinschi and J. Prasad, "A simulation model of an autonomous helicopter," Proceedings of RPV/UAV Systems Remotely Piloted Vehicles/Unmanned Airborne Vehicles, Bristol, UK, 1998.

[12] J. J. Dougherty, H. El-Sherief, D. J. Simon, and G. A. Whitmer, "GPS modeling for designing aerospace vehicle navigation systems," Aerospace and Electronic Systems, IEEE Transactions on, vol. 31, pp. 695-705, 1995. 
[13] X. Kong, "GPS Modeling in Frequency Domain," in Wireless Broadband and Ultra Wideband Communications, 2007. AusWireless 2007. The 2nd International Conference on, 2007, pp. 61-61.

[14] M. Mosavi, "Frequency domain modeling of gps positioning errors," in Signal Processing, 2006 8th International Conference on, 2006.

[15] E. M. Nebot, H. Durrant Whyte, and S. Scheding, "Frequency domain modeling of aided GPS for vehicle navigation systems," Robotics and Autonomous Systems, vol. 25, pp. 7382, 1998.

[16] M. Mosavi, "Modeling of GPS SPS timing error using multilayered neural network," in Signal Processing, 2006 8th International Conference on, 2006.

[17] L. Heng, G. X. Gao, T. Walter, and P. Enge, "Statistical characterization of GPS signal-inspace errors," in Proceedings of the 2011 International Technical Meeting of the Institute of Navigation (ION ITM 2011), San Diego, CA, 2011, pp. 312-319.

[18] L. Dong, "IF GPS signal simulator development and verification," UCGE Reports, 2003.

[19] S. H. Byun, G. A. Hajj, and L. E. Young, "Development and application of GPS signal multipath simulator," Radio Science, vol. 37, pp. 10-1-10-23, 2002.

[20] A. Brown, N. Gerein, and K. Taylor, "Modeling and simulation of GPS using software signal generation and digital signal reconstruction," in Proceedings of ION Technical Meeting, 2000.

[21] R. S. Conker, M. B. El-Arini, C. J. Hegarty, and T. Hsiao, "Modeling the effects of ionospheric scintillation on GPS/Satellite-Based Augmentation System availability," Radio Science, vol. 38, pp. 1-1-1-23, 2003.

[22] T.-K. Yeh, C. Hwang, G. Xu, C.-S. Wang, and C.-C. Lee, "Determination of global positioning system (GPS) receiver clock errors: impact on positioning accuracy," Measurement Science and Technology, vol. 20, p. 075105, 2009.

[23] J. Wang, B. H. Iz, and C. Lu, "Dependency of GPS positioning precision on station location," GPS Solutions, vol. 6, pp. 91-95, 2002.

[24] W. Y. Ochieng, K. Sauer, D. Walsh, G. Brodin, S. Griffin, and M. Denney, "GPS integrity and potential impact on aviation safety," Journal of Navigation, vol. 56, pp. 51-65, 2003. 
[25] U. I. Bhatti, "Improved integrity algorithms for integrated GPS/INS systems in the presence of slowly growing errors," Department of Civil and Environmental Engineering. Imperial College London, London, 2007.

[26] C. D. Milner and W. Y. Ochieng, "Failure Modes and Effects Analysis (FMEA) of GNSS Aviation Applications."

[27] E. Afraimovich, G. Zherebtsov, and G. Y. Smol'kov, "Total failure of GPS during a solar flare on December 6, 2006," in Doklady Earth Sciences, 2007, pp. 1231-1235.

[28] J. M. Dow, R. Neilan, and C. Rizos, "The international GNSS service in a changing landscape of global navigation satellite systems," Journal of Geodesy, vol. 83, pp. 191198, 2009.

[29] I. G. Service. IGS Product [Online]. Available: http://igscb.jpl.nasa.gov/components/prods.html

[30] J. Sanz, A. Rovira-Garcia, M. Hernandez-Pajares, J. Juan, J. Ventura-Traveset, C. LopezEchazarreta, et al., "The ESA/UPC GNSS-Lab Tool (gLAB)."

[31] P. Misra and P. Enge, "Special issue on global positioning system," Proceedings of the IEEE, vol. 87, pp. 3-15, 1999.

[32] G. P. P. Standard, ed, 2007.

[33] E.-R. Ahmed, "Introduction to GPS: the global positioning system," in Artech House communication series, Library of Congress, United States of America, 2002.

[34] G. Inc. (05). Available: http://sxbluegps.com/technology/gps-the-error-budget/

[35] (05/05). [online ]. Available: http://wiki.awf.forst.unigoettingen.de/wiki/index.php/Global Positioning System (GPS)

[36] G. Xu, GPS: theory, algorithms, and applications: Springer-Verlag Berlin Heidelberg, 2007.

[37] R. Prasad and M. Ruggieri, Applied satellite navigation using GPS, GALILEO, and augmentation systems: Artech House, 2005.

[38] R. Grisso. (07/20/2013). Precision Farming Tools. Available: http://pubs.ext.vt.edu/442/442-503/442-503.html 
[39] J. A. Arnold, "New applications make NDGPS more pervasive," Public Roads, vol. 64, 2001.

[40] SCADA. [Online]. Available: http://tech.nakedmaya.com/2008/07/sources-of-errors-ingps/

[41] M. G. Perhinschi, H. Moncayo, J. Davis, B. Wilburn, O. Karas, and M. Wathen, "Development of a simulation environment for autonomous flight control algorithms," in Proceedings from AIAA Modeling and Simulation Technologies Conference, Portland, OR, AIAA, 2011, p. 2011.

[42] O. Karas, "UAV Simulation Environment for Autonomous Flight Control Algorithms," 1520729 M.S., West Virginia University, 2012.

[43] Y. Gu, B. Seanor, G. Campa, M. R. Napolitano, L. Rowe, S. Gururajan, et al., "Design and flight testing evaluation of formation control laws," Control Systems Technology, IEEE Transactions on, vol. 14, pp. 1105-1112, 2006.

[44] G. Campa, Y. Gu, B. Seanor, M. R. Napolitano, L. Pollini, and M. L. Fravolini, "Design and flight-testing of non-linear formation control laws," Control Engineering Practice, vol. 15, pp. 1077-1092, 2007.

[45] H. Moncayo, M. G. Perhinschi, B. Wilburn, J. Wilburn, and O. Karas, "Extended Nonlinear Dynamic Inversion Control Laws for Unmanned Air Vehicles," 2012.

[46] H. Moncayo, M. G. Perhinschi, B. Wilburn, J. Wilburn, and O. Karas, "UAV Adaptive Control Laws Using Non-Linear Dynamic Inversion Augmented with an Immunity-based Mechanism," 2012.

[47] B. K. Wilburn, M. G. Perhinschi, H. Moncayo, O. Karas, and J. N. Wilburn, "Unmanned Aerial Vehicle Trajectory Tracking Algorithm Comparison," International Journal of Intelligent Unmanned Systems, vol. 1, pp. 6-6, 2013. 